Supporting Information

\title{
Regiodiscriminating Reactivity of Isolable NHC-Coordinated Disilenyl Germylene and Its Cyclic Isomer
}

David Nieder ${ }^{\dagger}$, Volker Huch ${ }^{\dagger}$, Cem B. Yildiz** and David Scheschkewitz*, ${ }^{*}$

\section{Contents}

1. Plots of NMR spectra S-2

2. UV/vis spectra $\quad S-21$

3. Computational details S-30

4. References S-134

${ }^{\dagger}$ Krupp-Chair of General and Inorganic Chemistry, Saarland University, 66125 Saarbrücken, Germany

$\ddagger^{\ddagger}$ Department of Chemistry, Faculty of Science and Letters, Aksaray University, 68100 Aksaray, Turkey

E-mail:

* scheschkewitz@mx-uni-saarland.de

* cemburakyildiz@aksaray.edu.tr 


\section{Plots of NMR spectra}

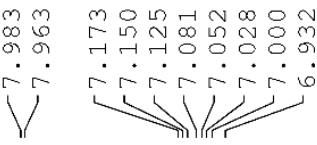

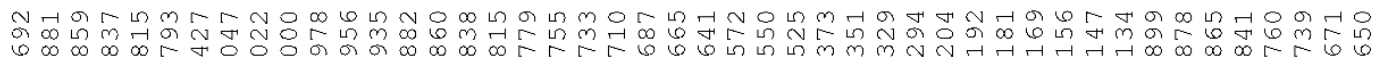

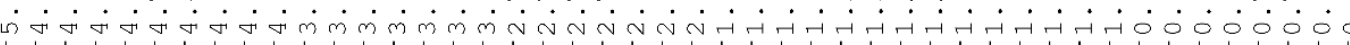
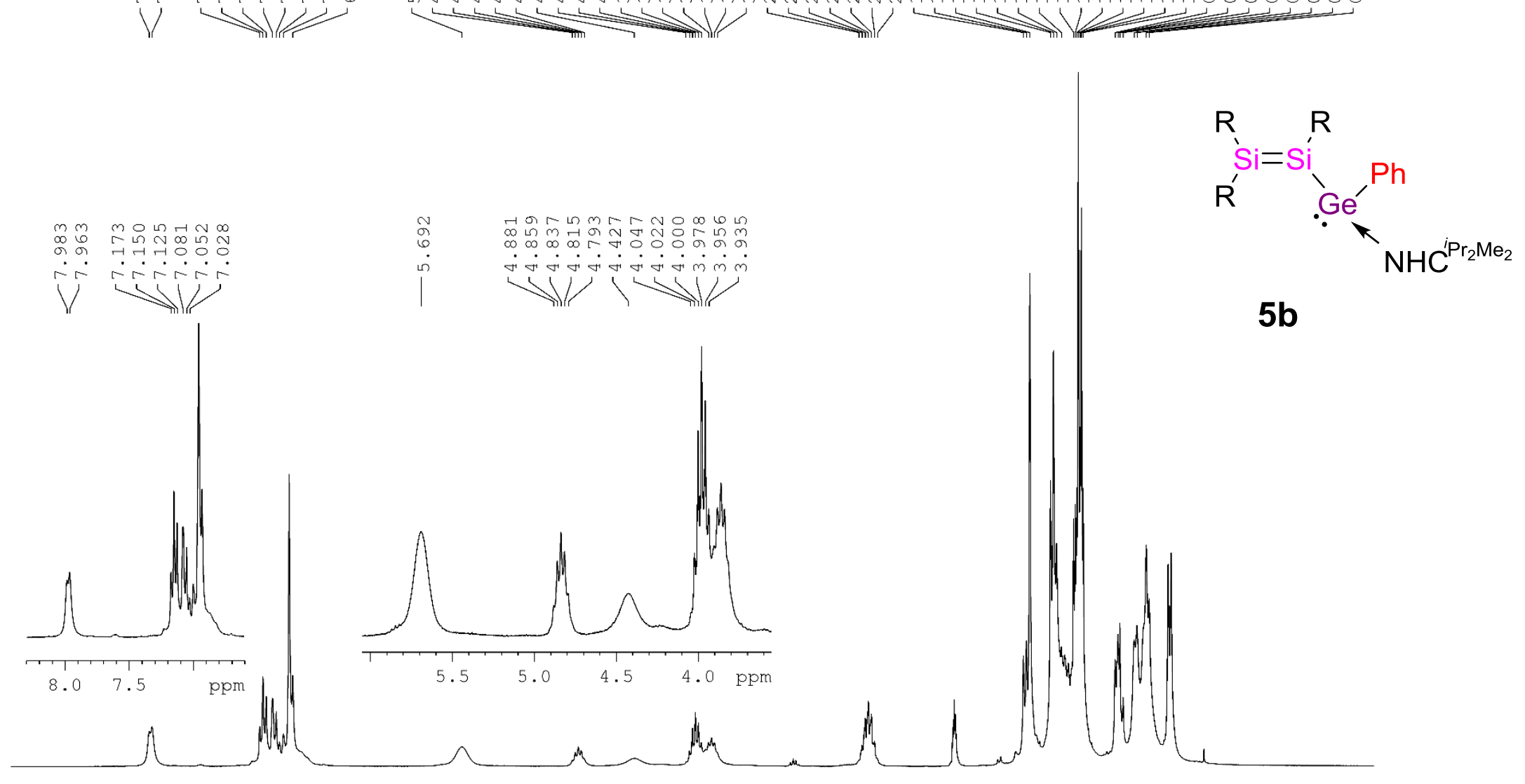

$8.5 \quad 8.0$
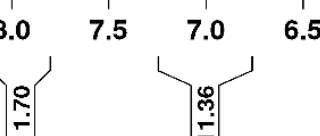

Figure S1: ${ }^{1} \mathrm{H}$ NMR of $\mathbf{5 b}$ in toluene- $d^{4}$ at $300 \mathrm{~K}$.

$5 b$ 


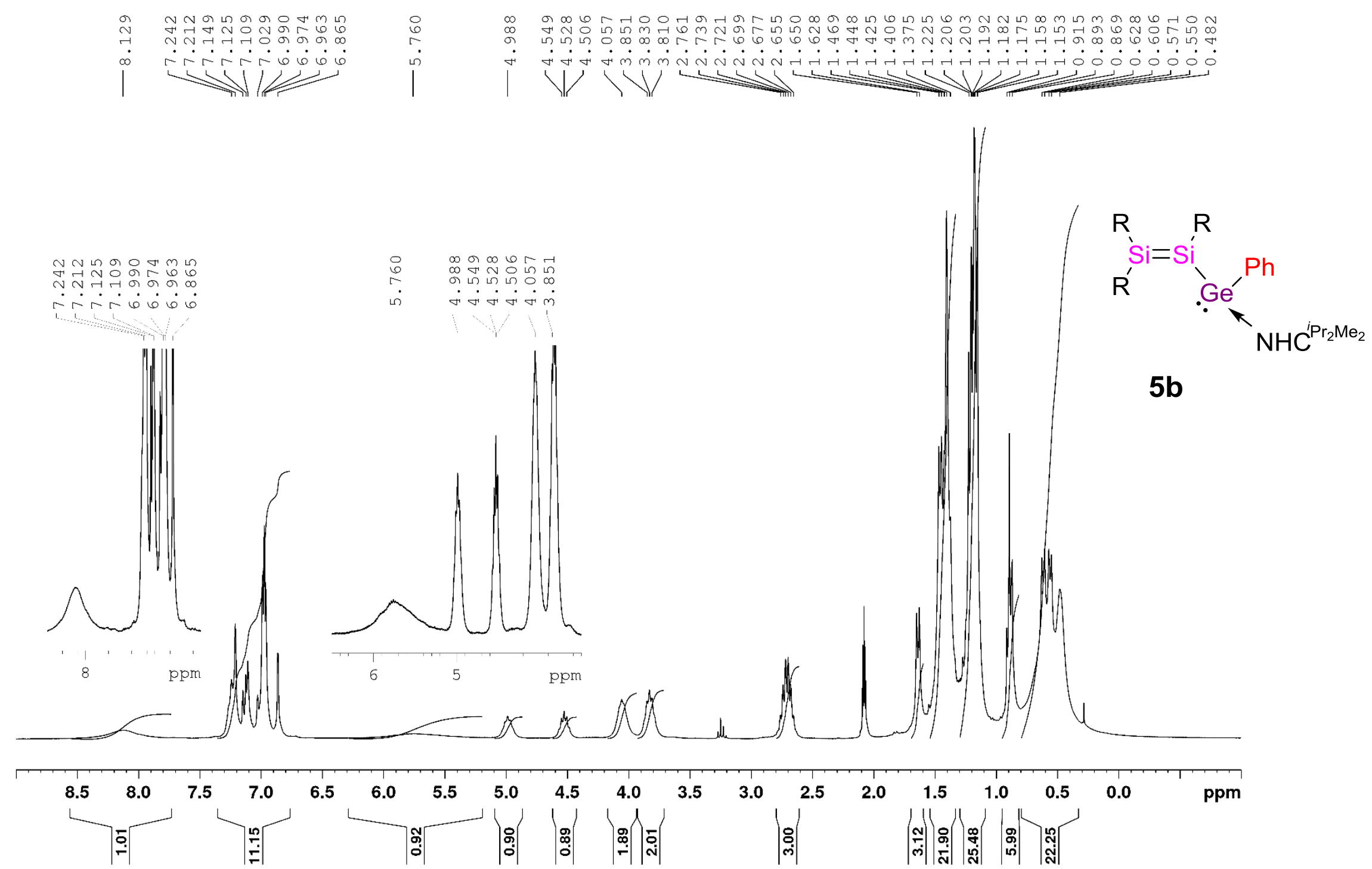

Figure S2: ${ }^{1} \mathrm{H}$ NMR of $\mathbf{5 b}$ in toluene- $d^{\breve{5}}$ at $253 \mathrm{~K}$. 


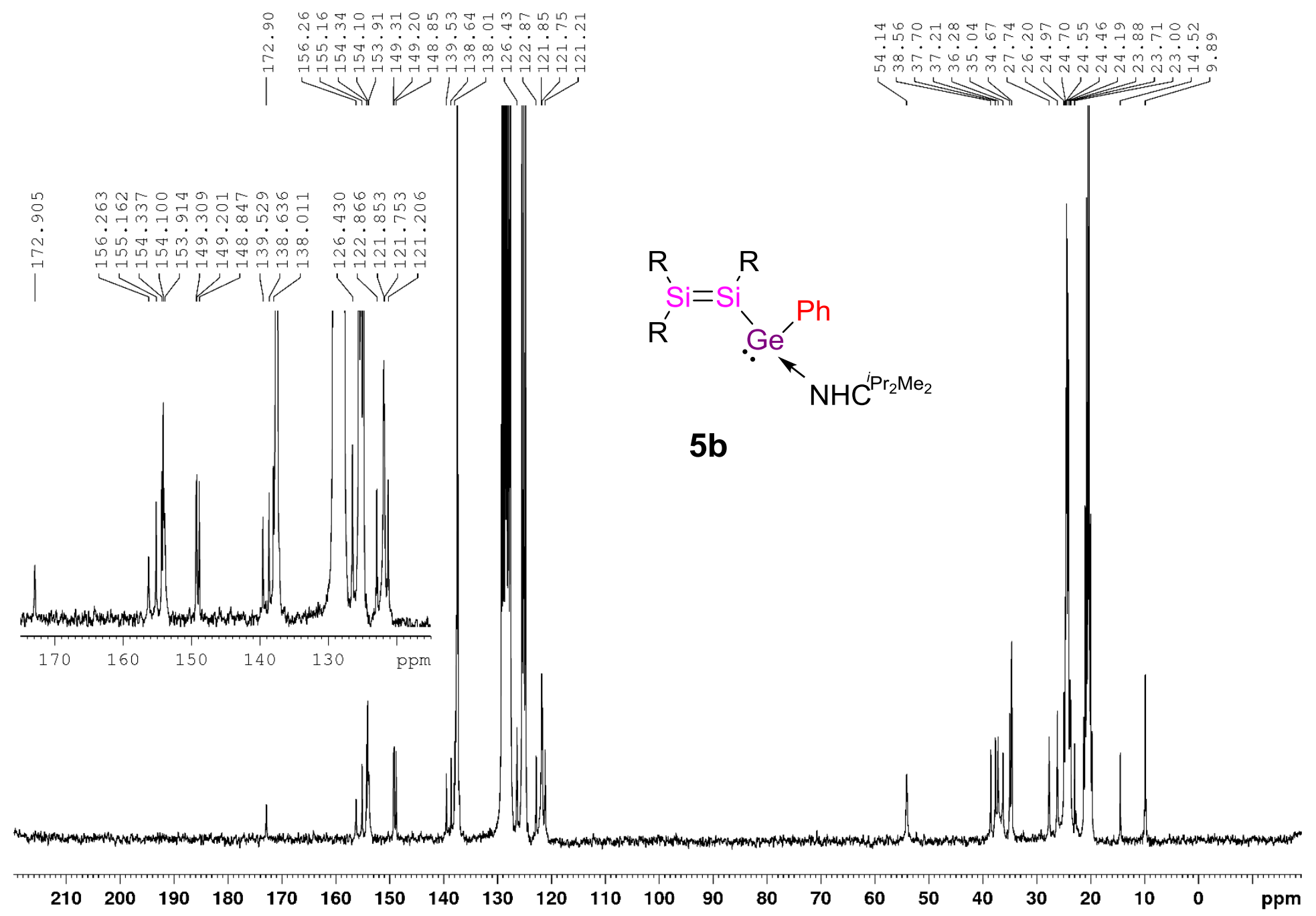

Figure S3: ${ }^{13} \mathrm{C}$ NMR of $5 \mathbf{b}$ in toluene- $d^{8}$ at $253 \mathrm{~K}$. 


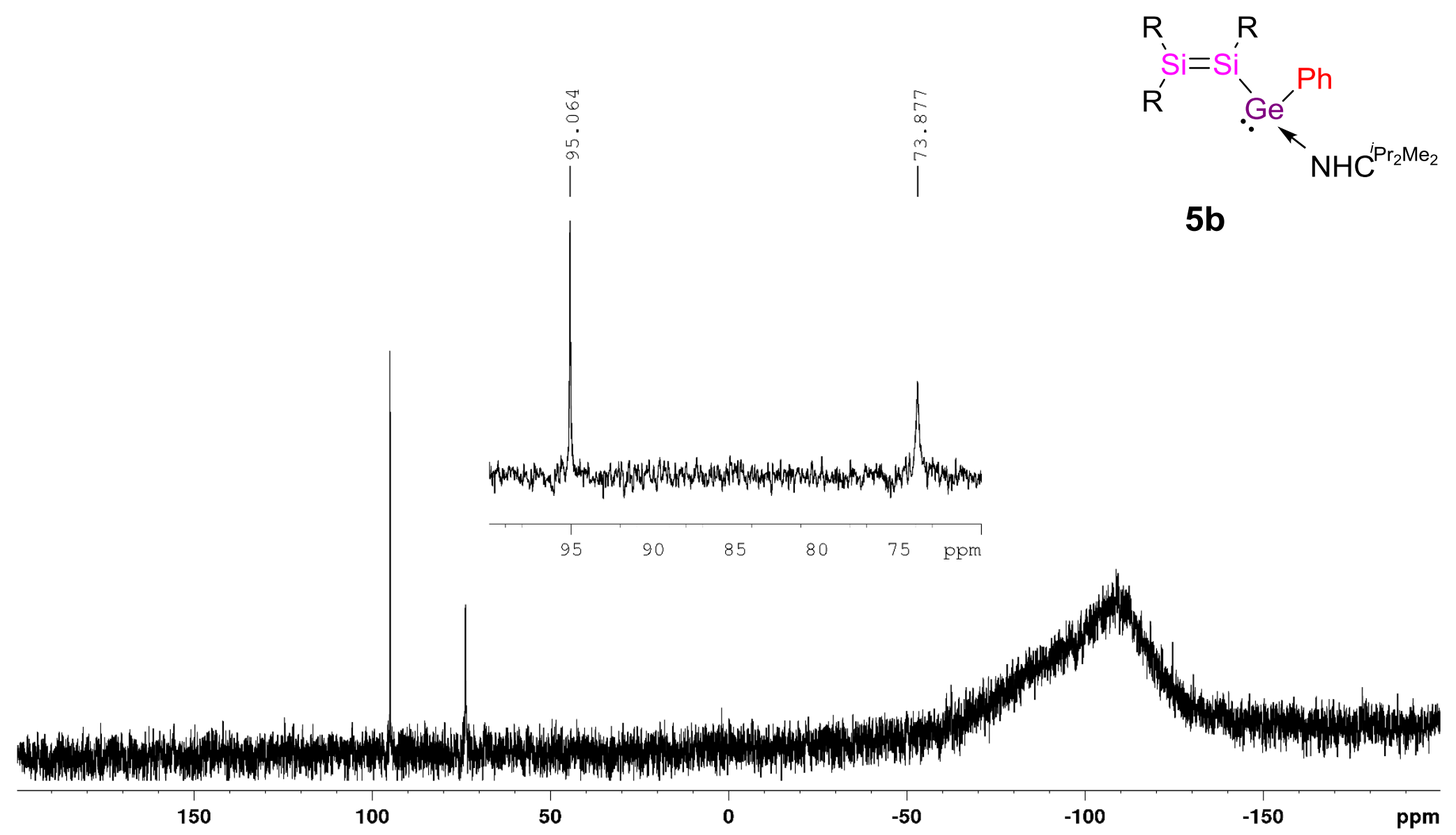

Figure S4: ${ }^{2 y} \mathrm{Si}$ NMR of $\mathbf{5 b}$ in toluene- $d^{4}$ at $253 \mathrm{~K}$. 


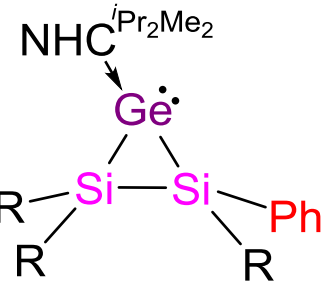

$6 b$

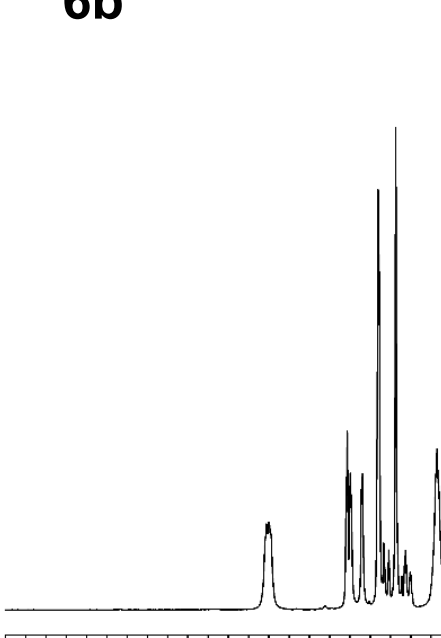

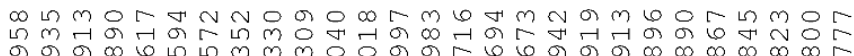

"
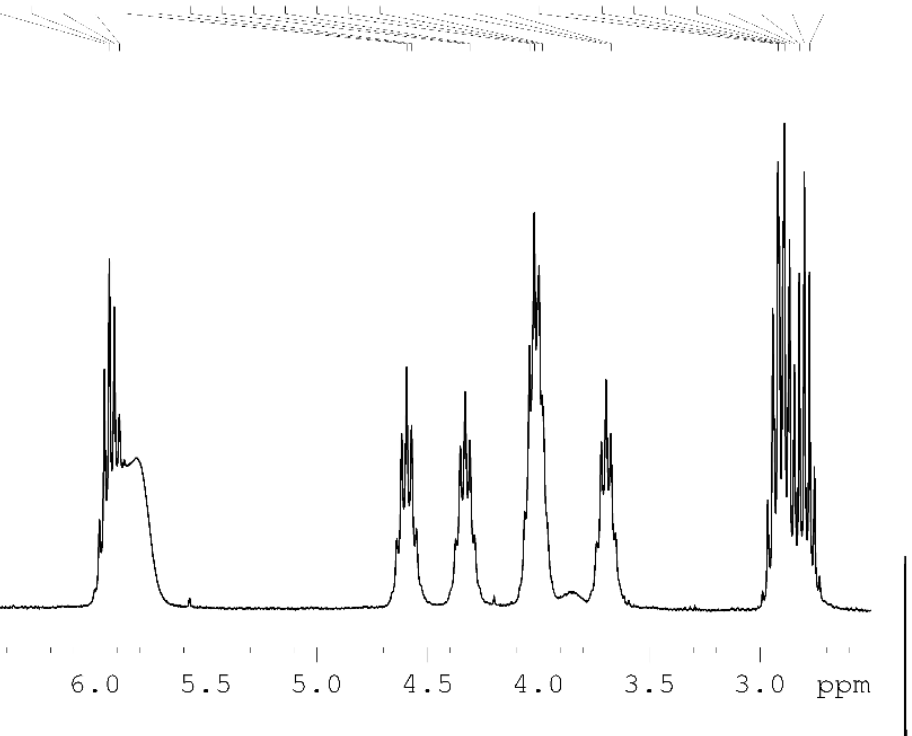

Wh

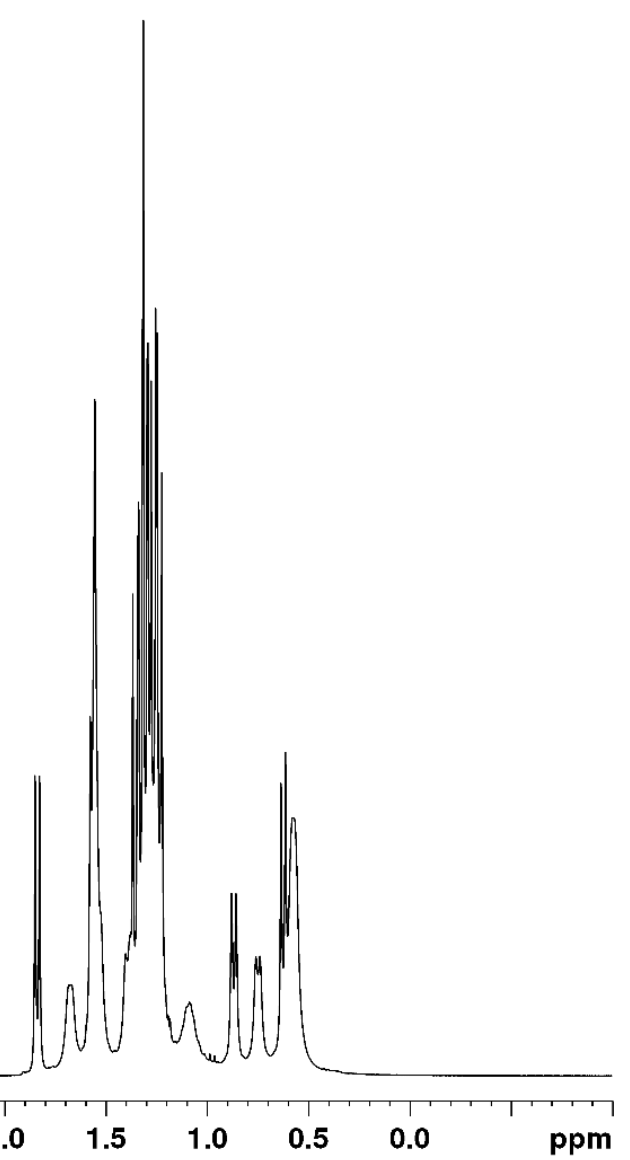
(.) 1040
|
ฟิ่
量|

$\begin{array}{lll}4.5 & 4.0 & 3.5\end{array}$

Figure S5: ${ }^{1} \mathrm{H}$ NMR of $6 b$ in $\left[D_{6}\right]$-benzene at $300 \mathrm{~K}$. 


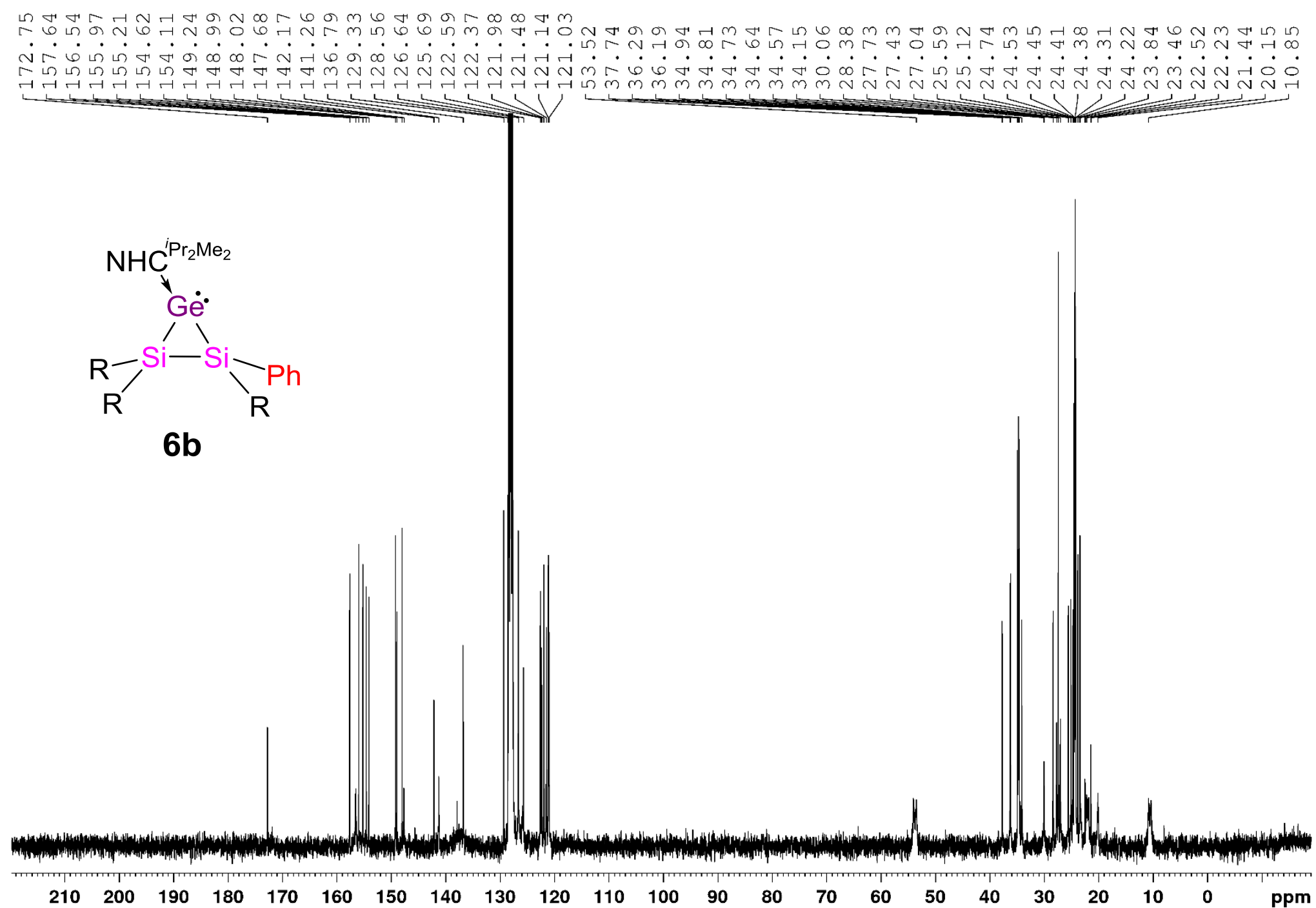

Figure $\mathbf{S 6 :}{ }^{13} \mathrm{C}$ NMR of $6 \mathbf{b}$ in $\left[\mathrm{D}_{6}\right]$-benzene at $300 \mathrm{~K}$. 


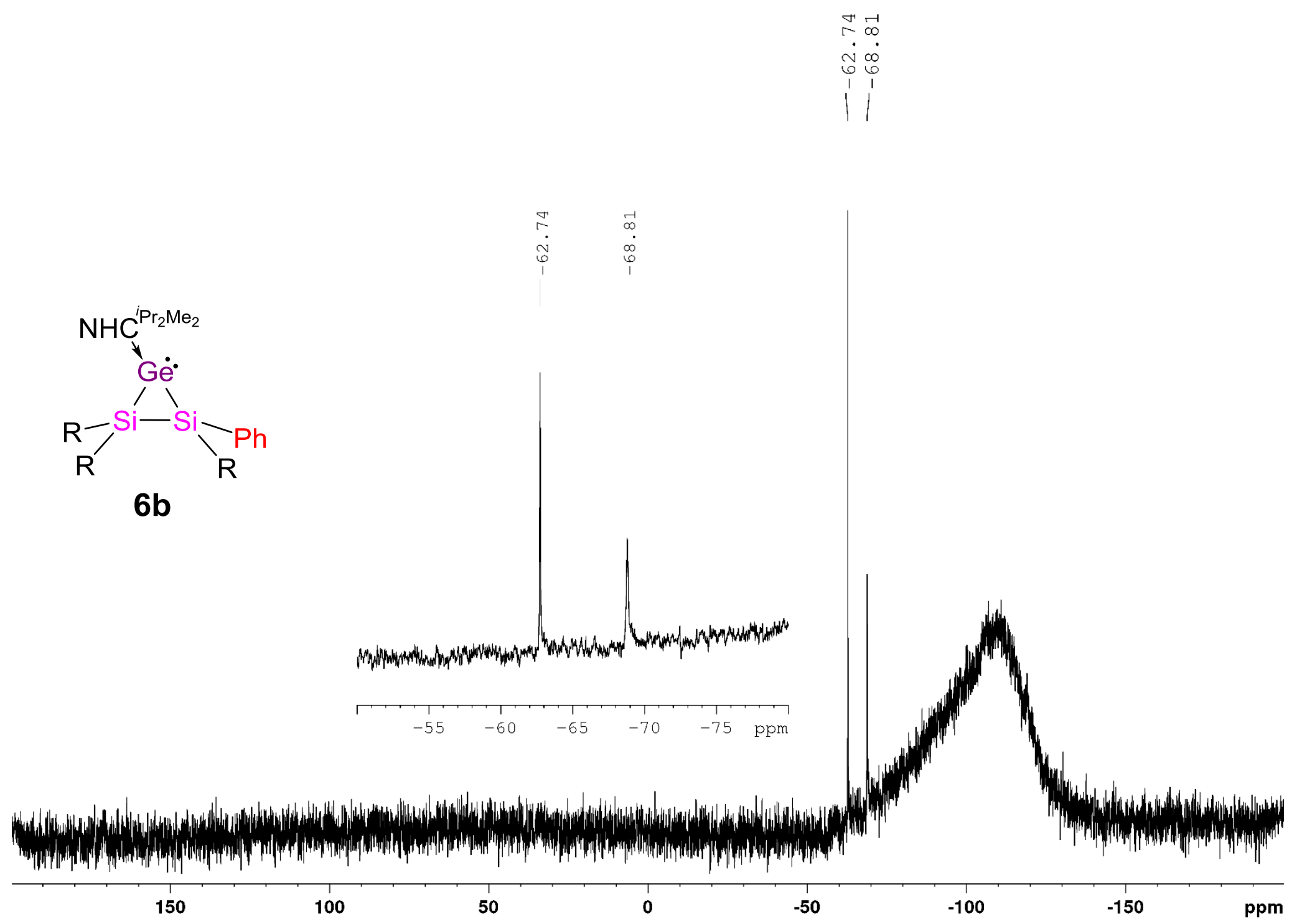

Figure S7: ${ }^{2 y} \mathrm{Si}$ NMR of $\mathbf{6 b}$ in $\left[\mathrm{D}_{6}\right]$-benzene at $300 \mathrm{~K}$. 


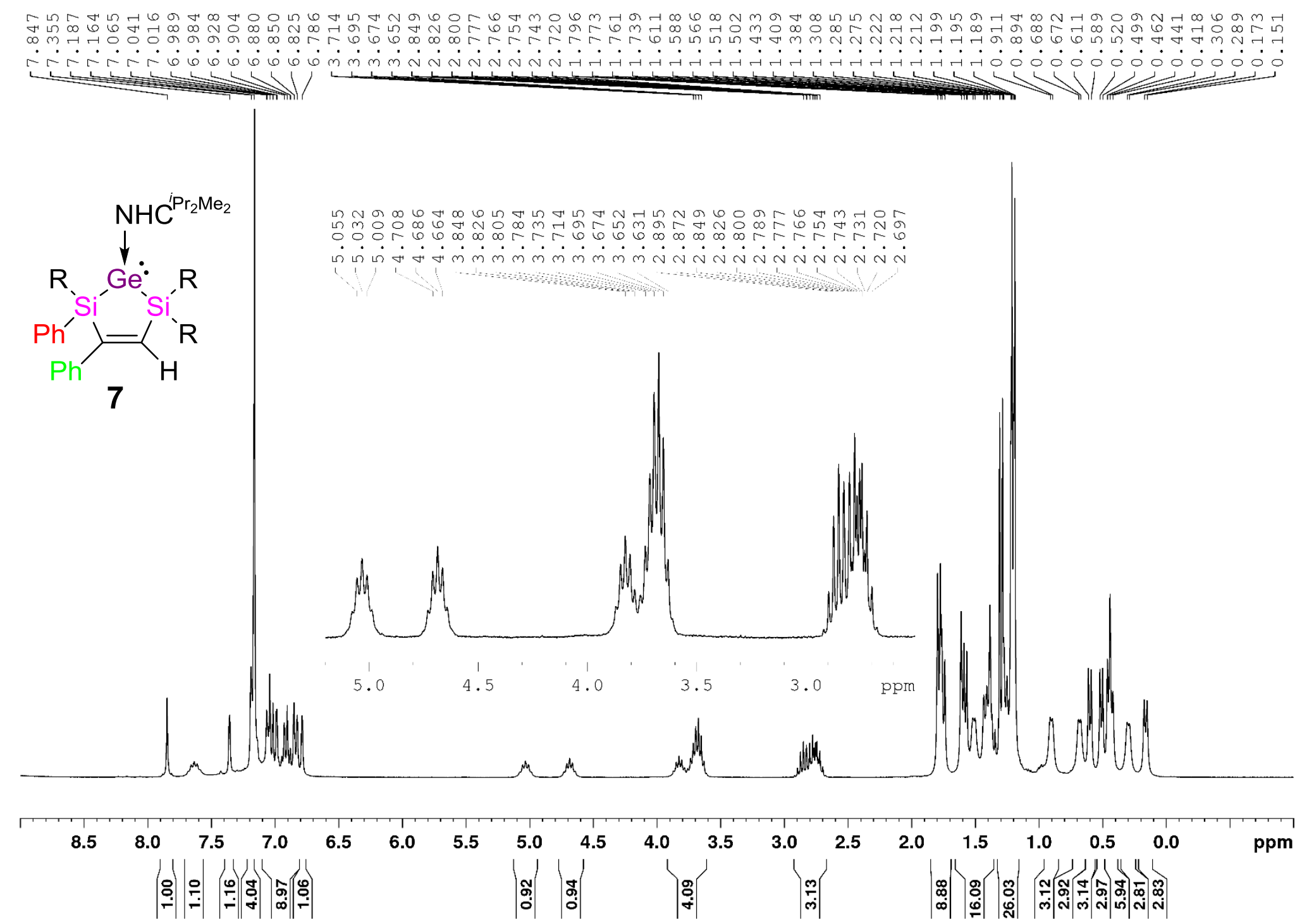

Figure S8: ${ }^{1} \mathrm{H}$ NMR of 7 in $\left[\mathrm{D}_{6}\right]$-benzene at $300 \mathrm{~K}$. 


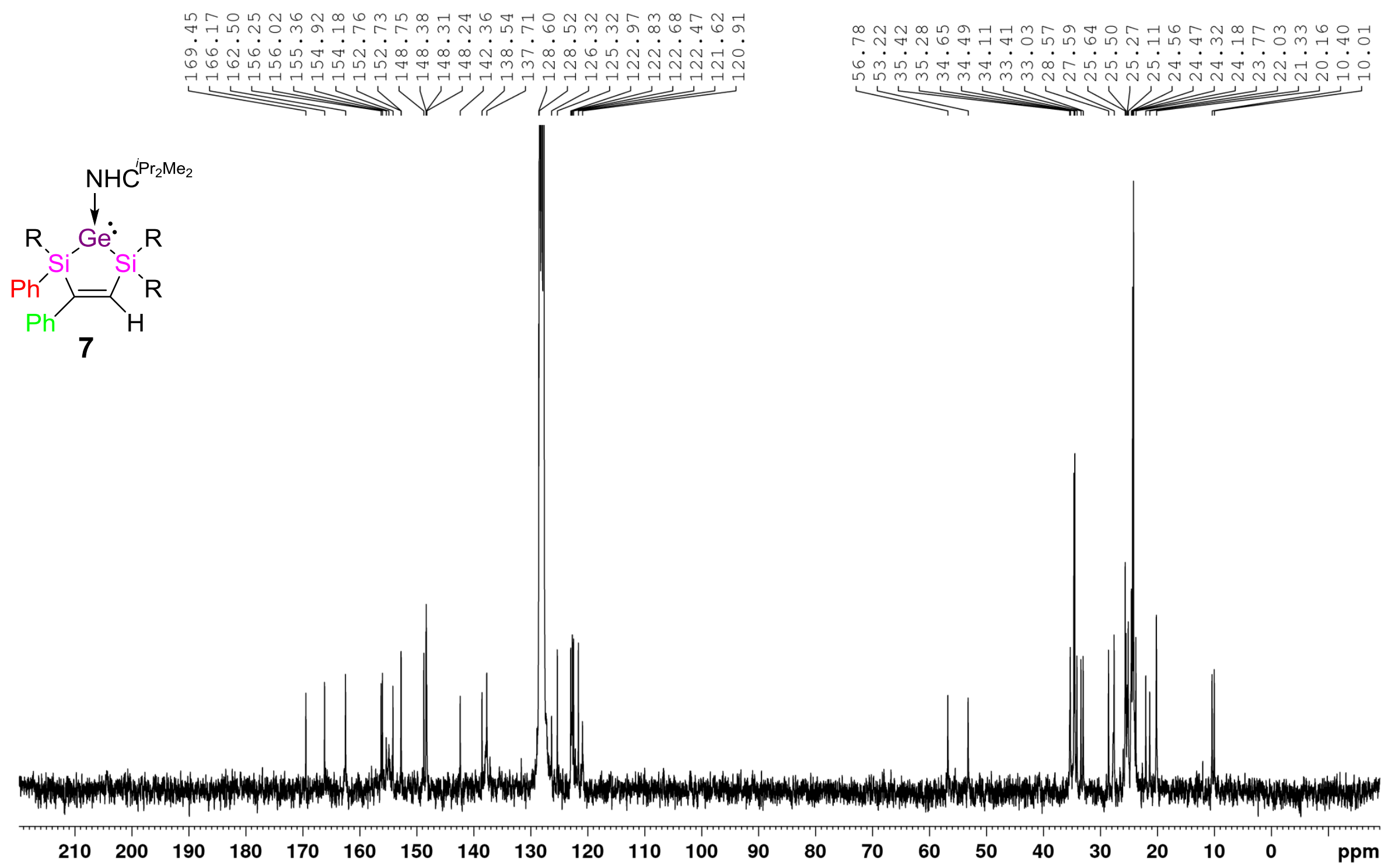

Figure S9: ${ }^{13} \mathrm{C}$ NMR of 7 in $\left[D_{6}\right]$-benzene at $300 \mathrm{~K}$. 


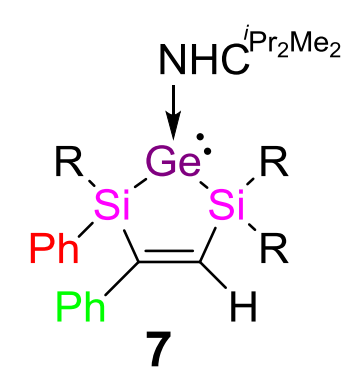

s

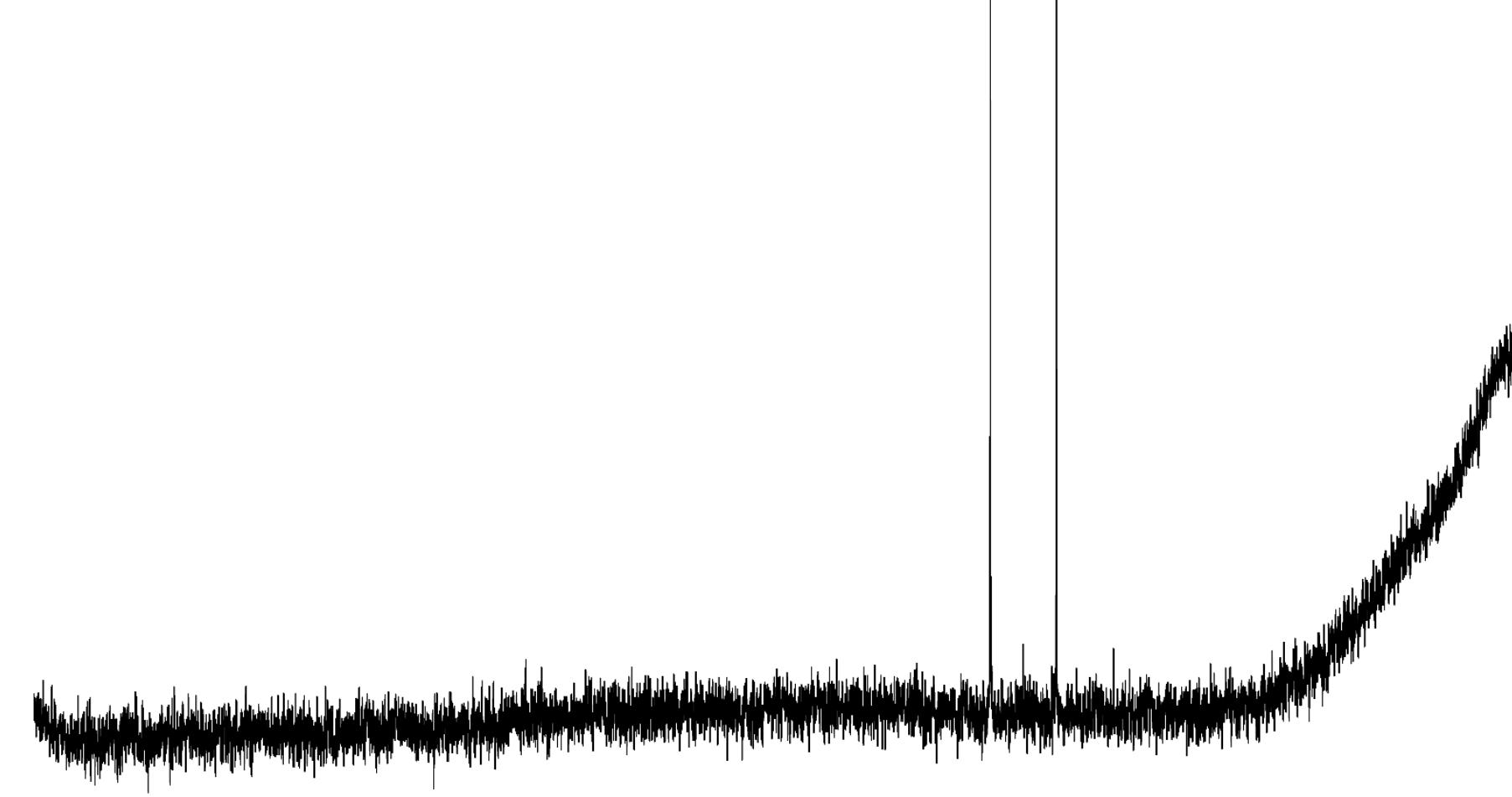

$150 \quad 100$

Figure S10: ${ }^{2 y} \mathrm{Si} N \mathrm{NM}$ of 7 in $\left[\mathrm{D}_{6}\right]$-benzene at $300 \mathrm{~K}$. 

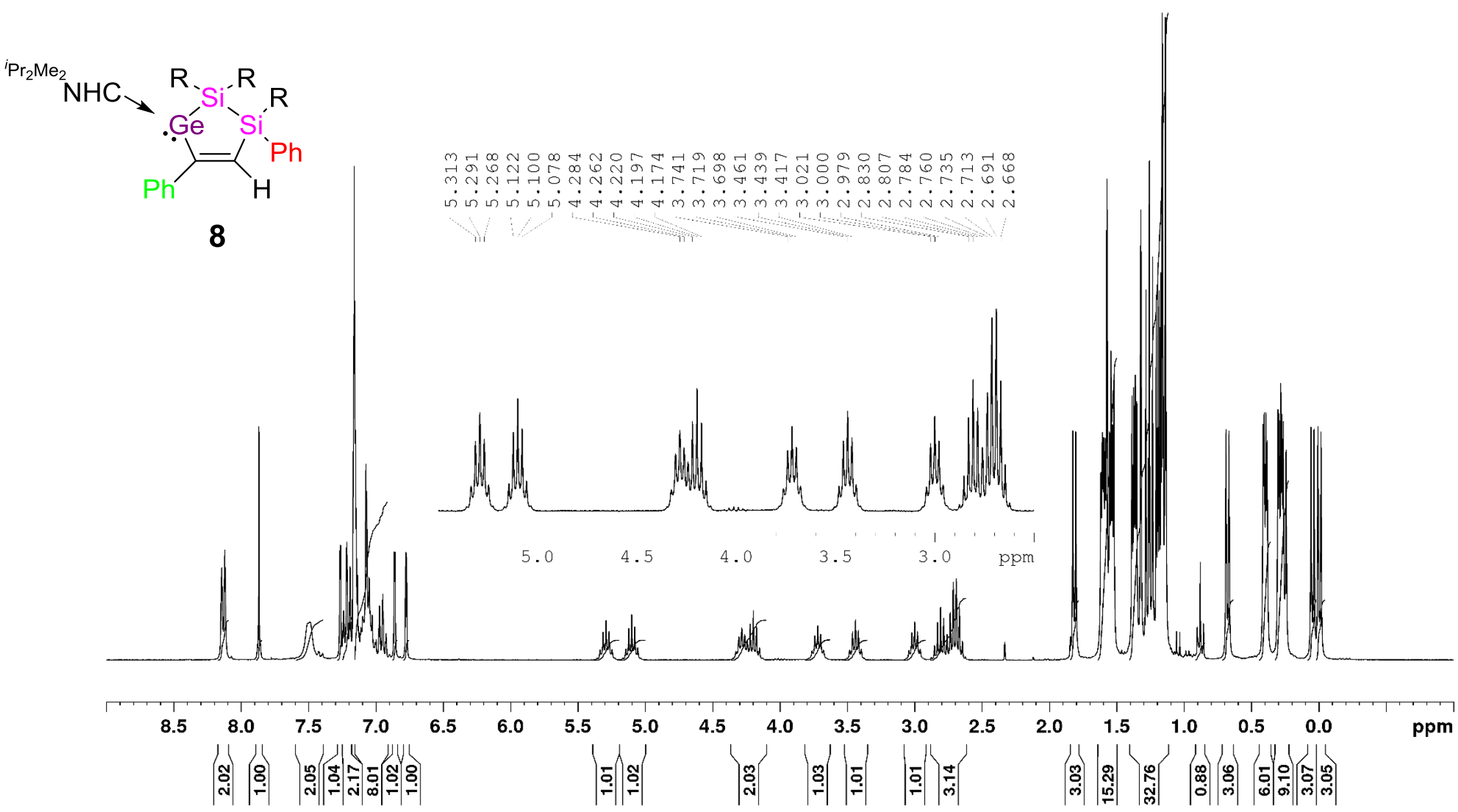

Figure S11: ${ }^{1} \mathrm{H}$ NMR of 8 in $\left[D_{6}\right]$-benzene at $300 \mathrm{~K}$. 


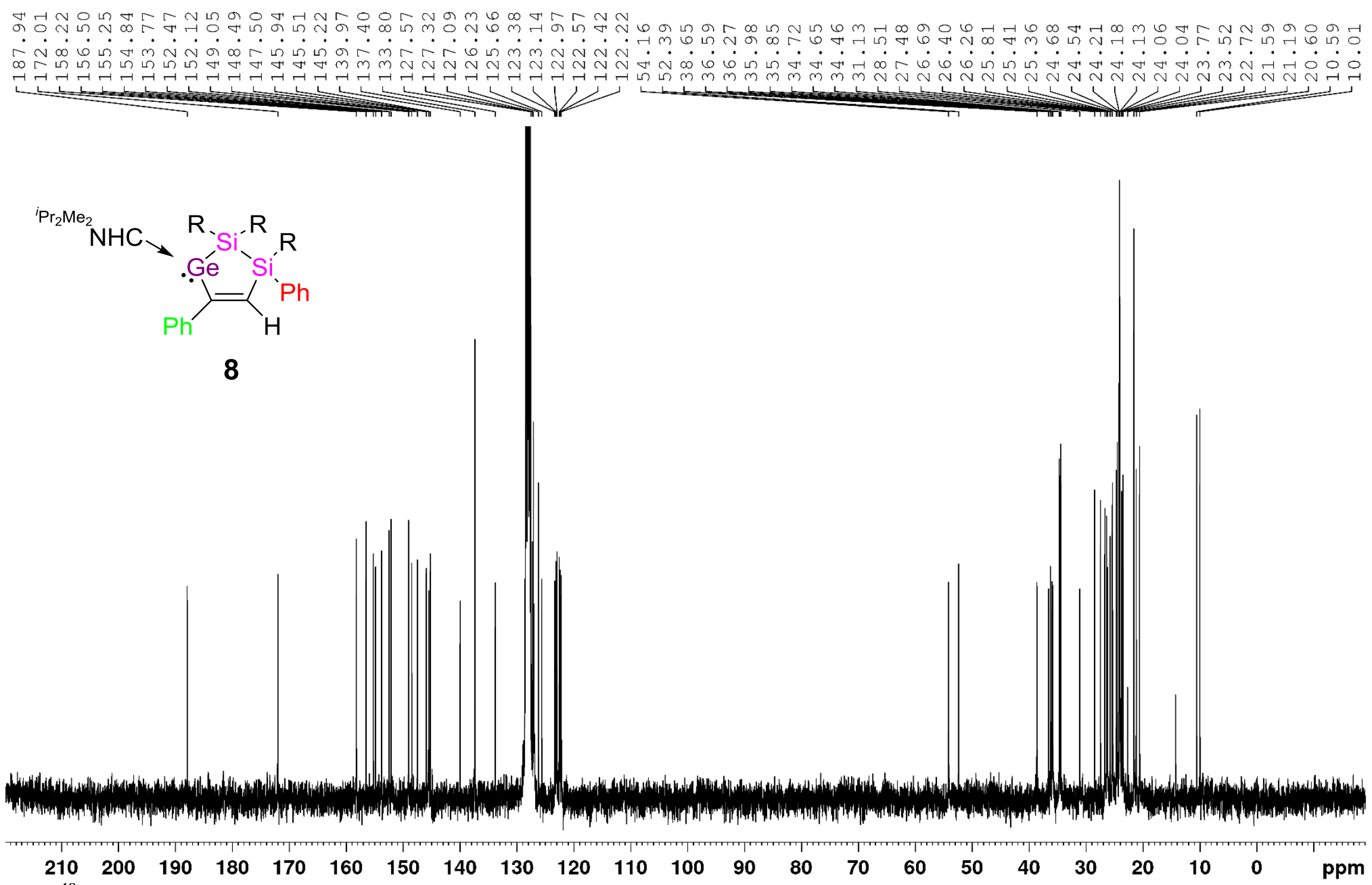

Figure S12: ${ }^{13} \mathrm{C}$ NMR of 8 in $\left[\mathrm{D}_{6}\right]$-benzene at $300 \mathrm{~K}$. 


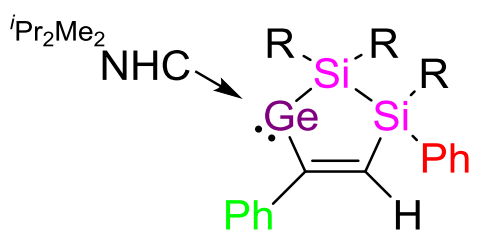

8

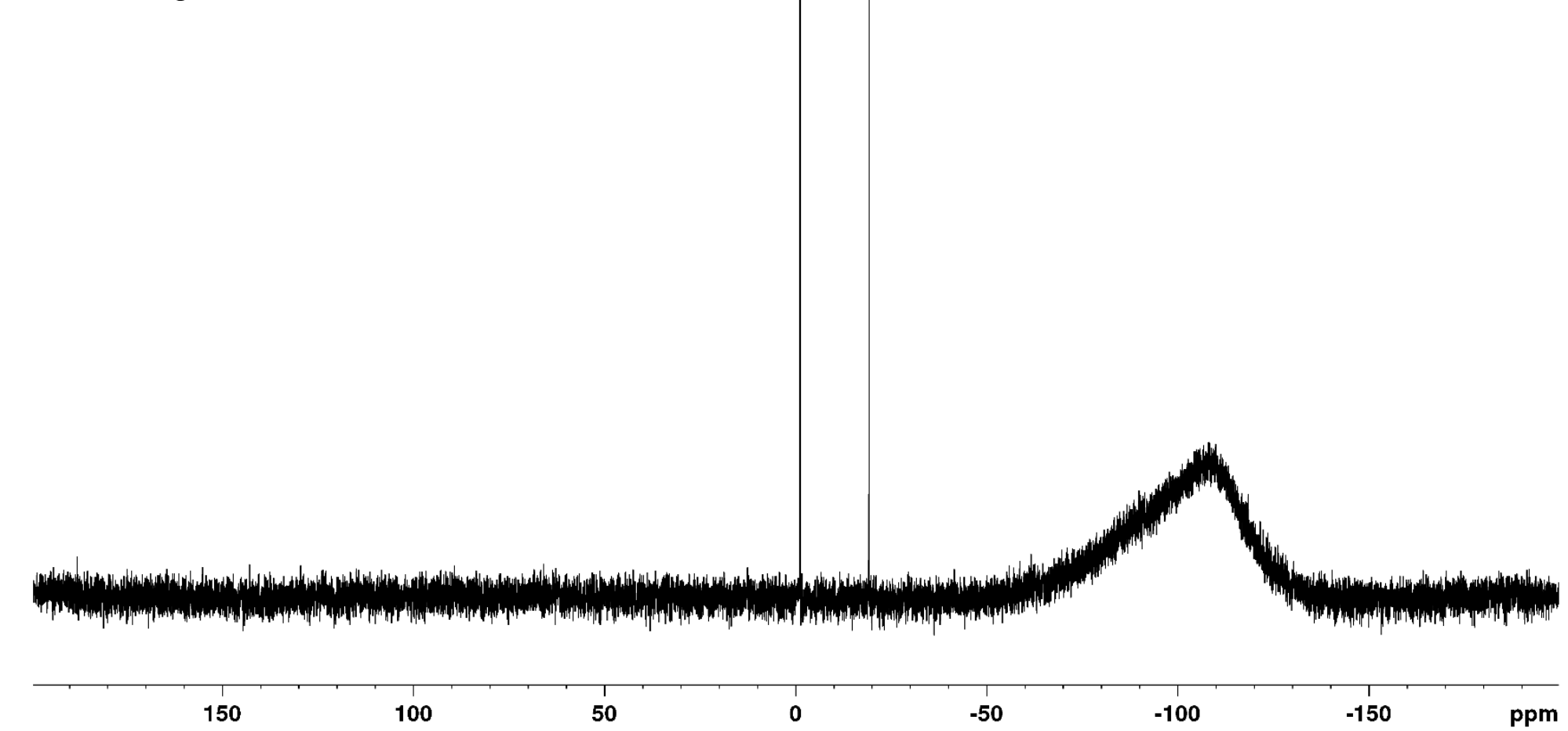

Figure S13: ${ }^{2 y} \mathrm{Si}$ NMR of 8 in $\left[\mathrm{D}_{6}\right]$-benzene at $300 \mathrm{~K}$. 


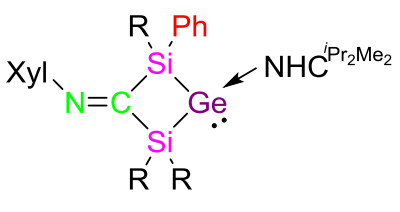

12

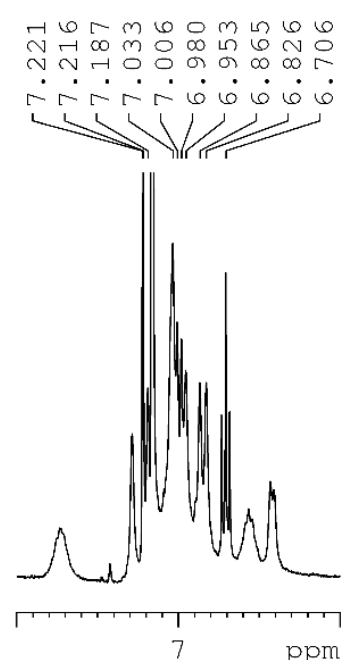

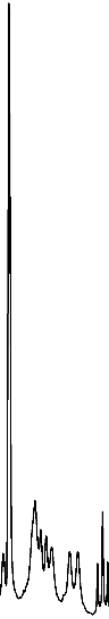
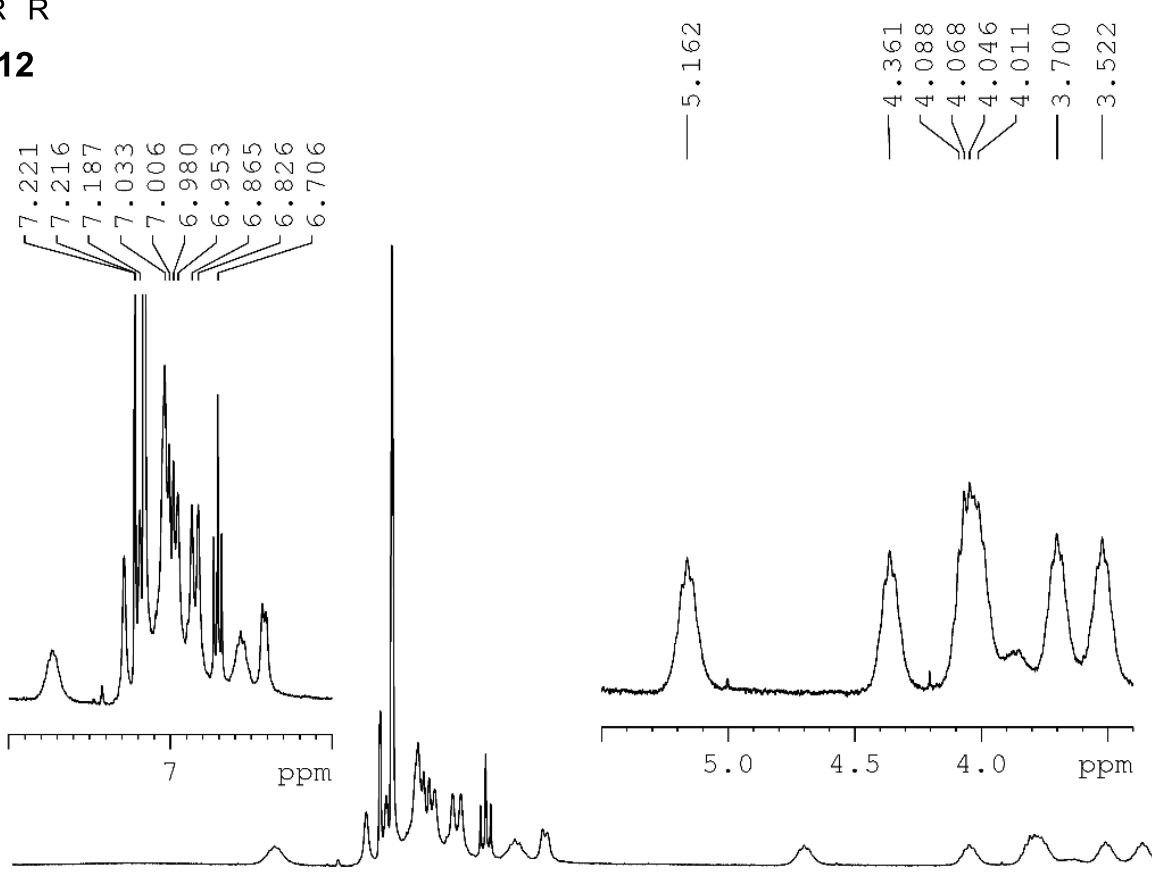

8.5
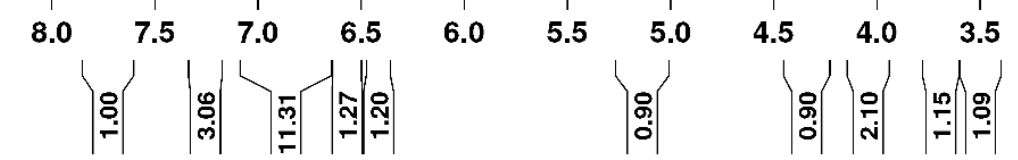

Figure S14: ' $\mathrm{H}$ NMR of 12 in $\left[\mathrm{D}_{6}\right]$-benzene at $300 \mathrm{~K}$. 


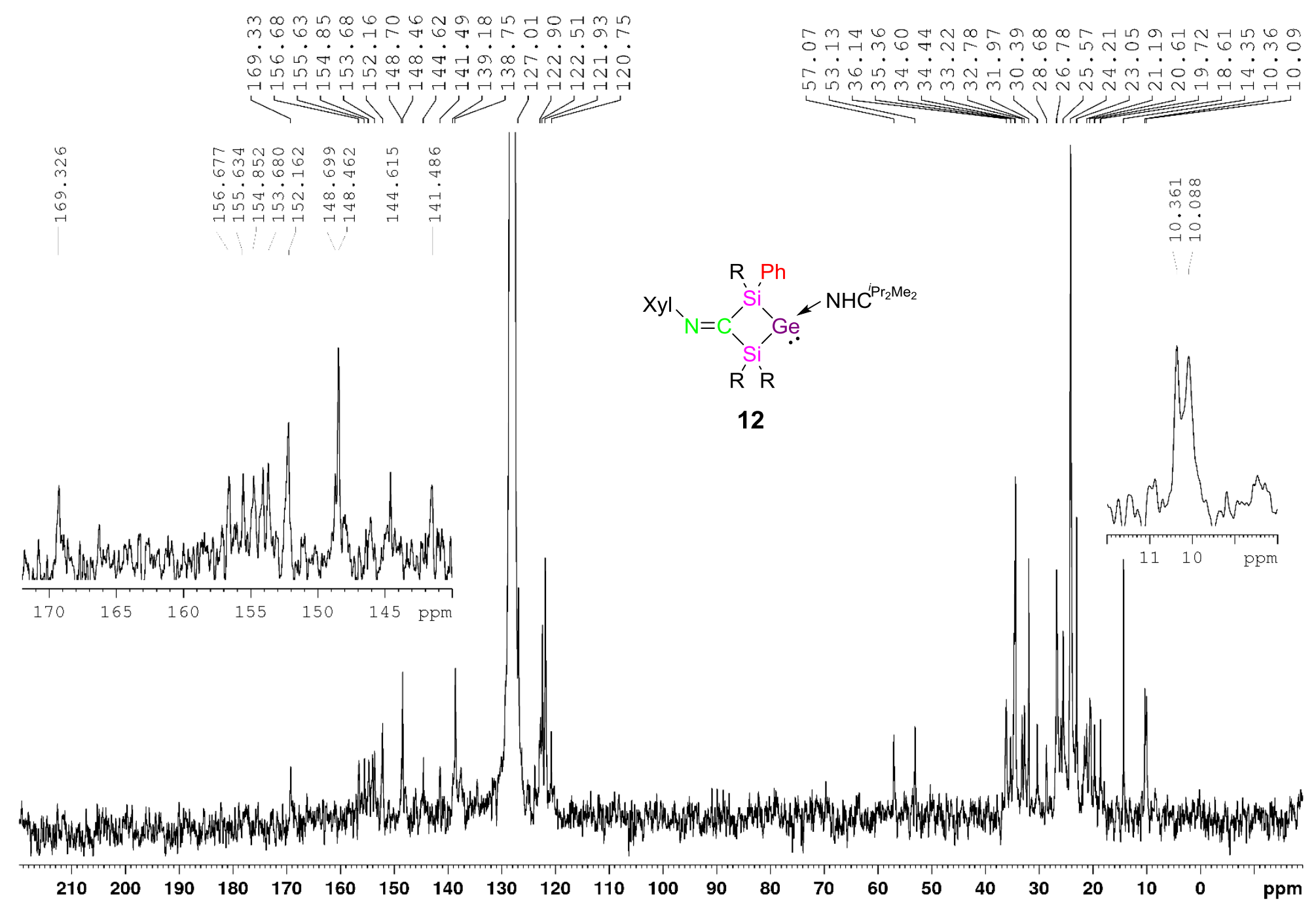

Figure S15: ${ }^{13} \mathrm{C}$ NMR of 12 in $\left[D_{6}\right]$-benzene at $300 \mathrm{~K}$. 


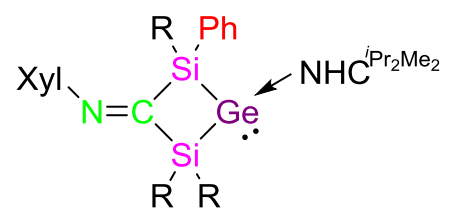

12

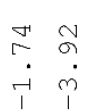
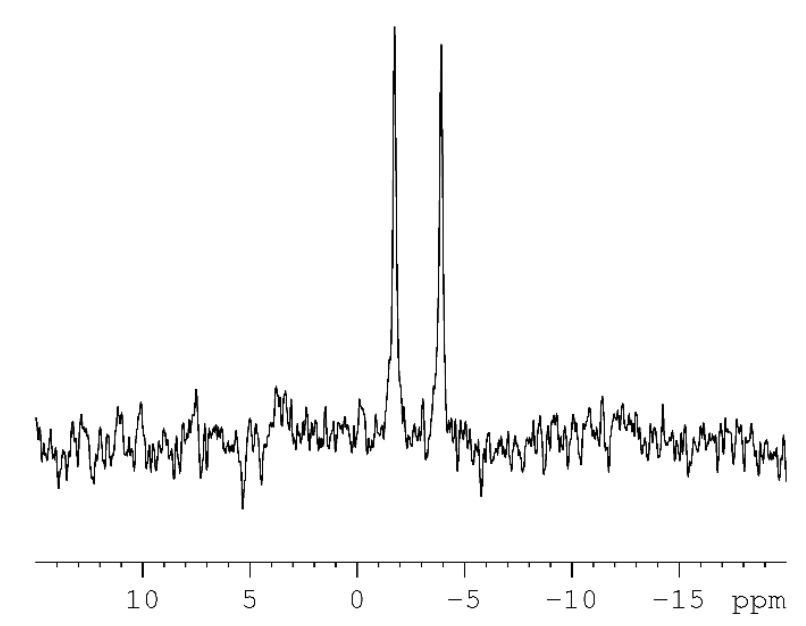

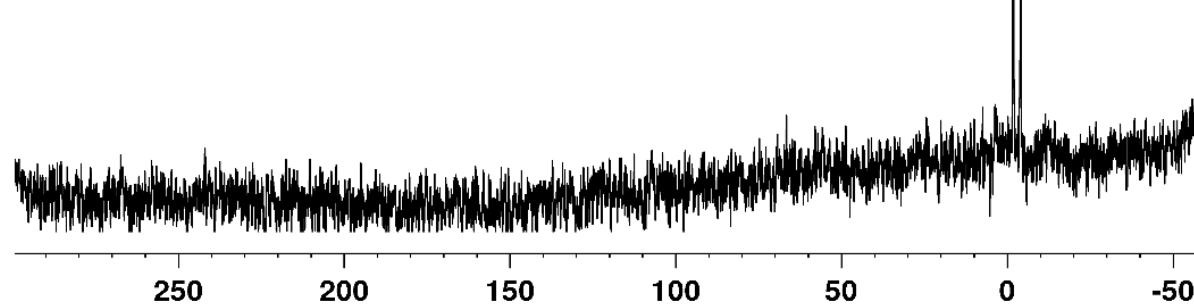

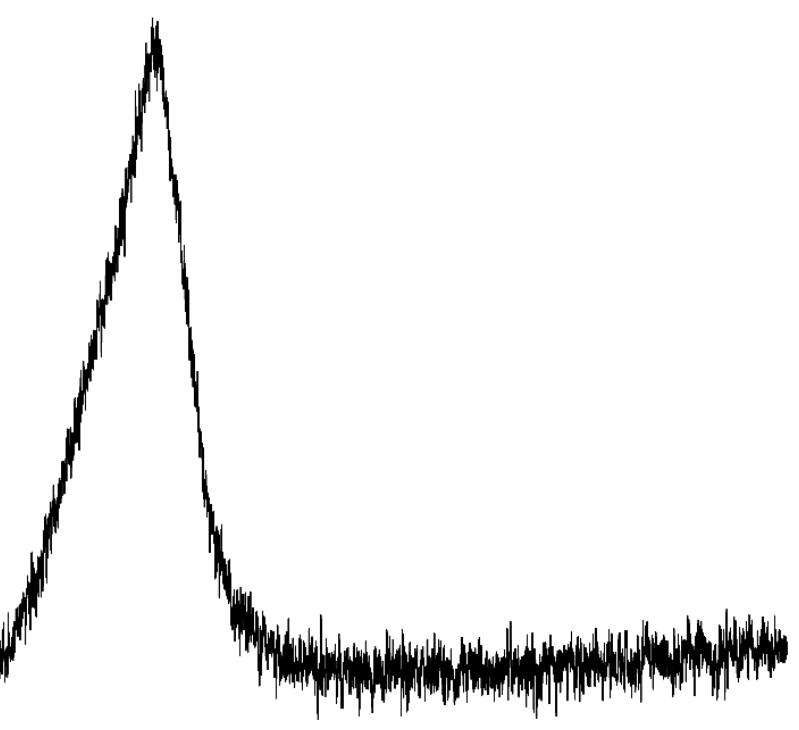

150

100

50

0

$-50$

$-100$

$-150$

$-200$

$-250$

ppm

Figure S16: ${ }^{29} \mathrm{Si} N M R$ of 12 in $\left[D_{6}\right]$-benzene at $300 \mathrm{~K}$. 


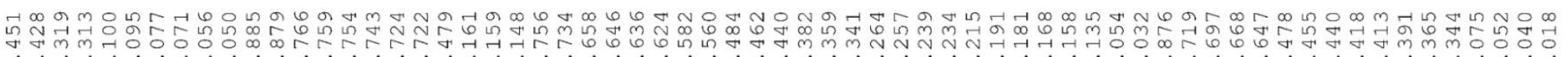

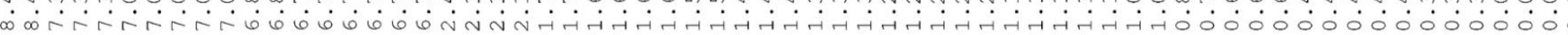

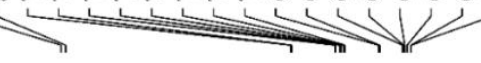

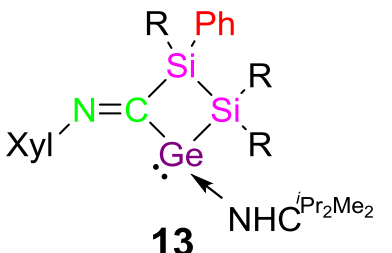

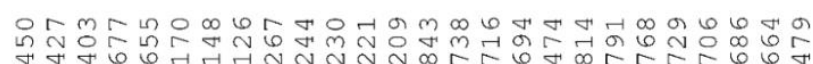

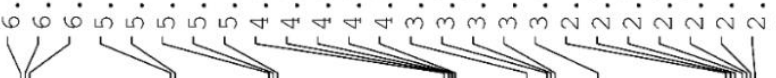

13
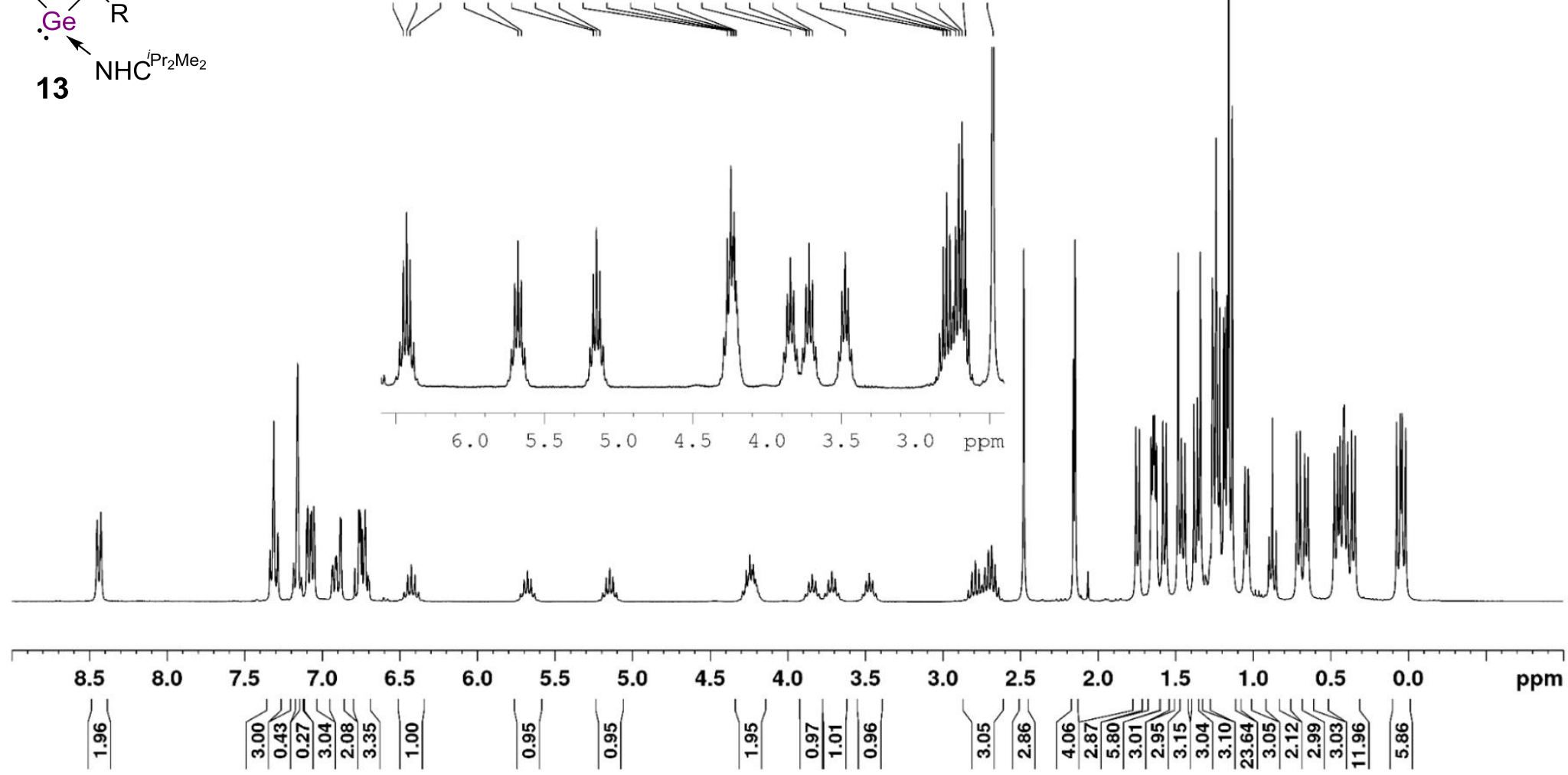

Figure S17: 'H NMR of 13 in $\left[D_{6}\right]$-benzene at $300 \mathrm{~K}$. 

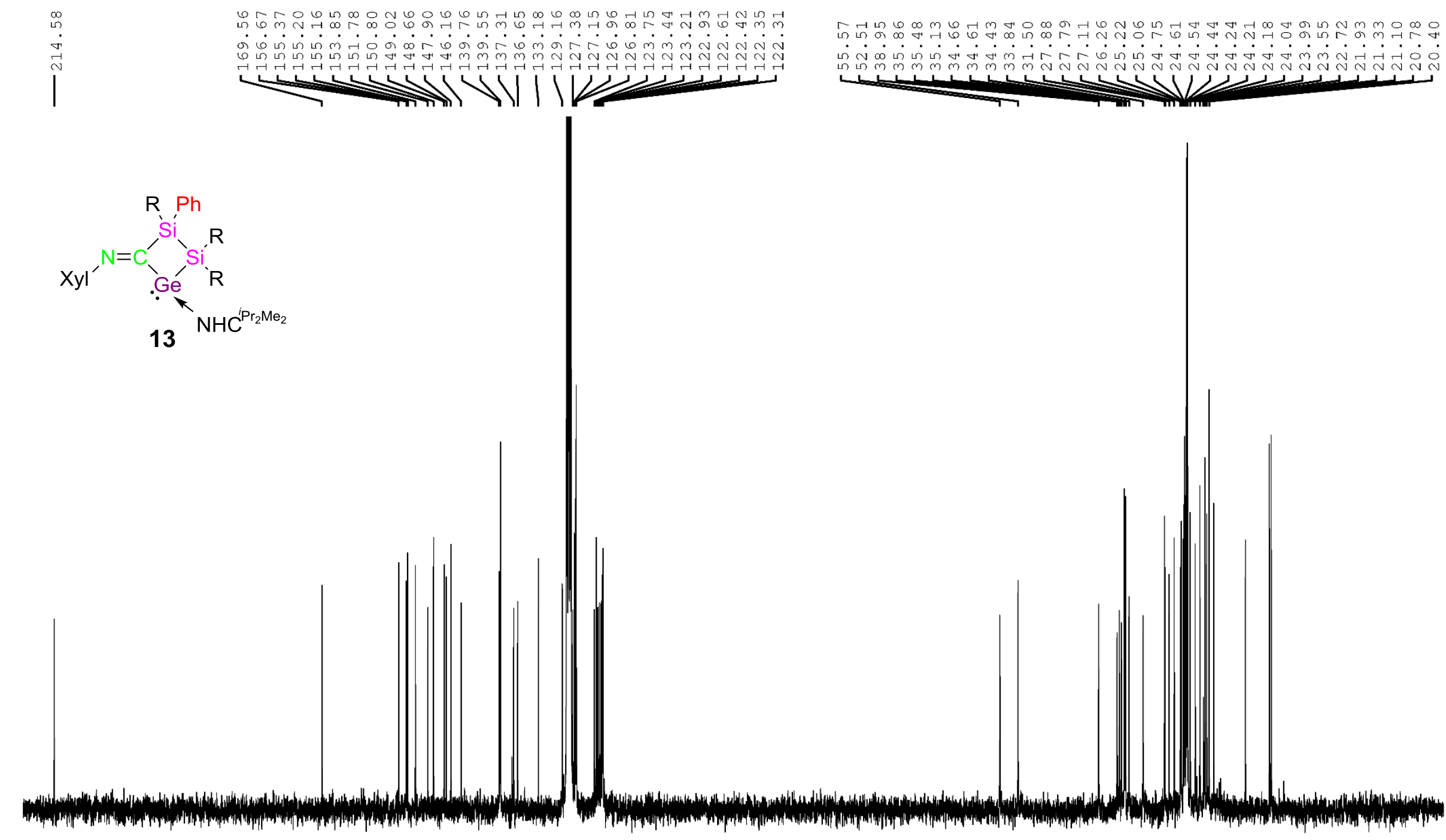

$\begin{array}{lllllllll}210 & 200 & 190 & 180 & 170 & 160 & 150 & 140 & 130\end{array}$ 


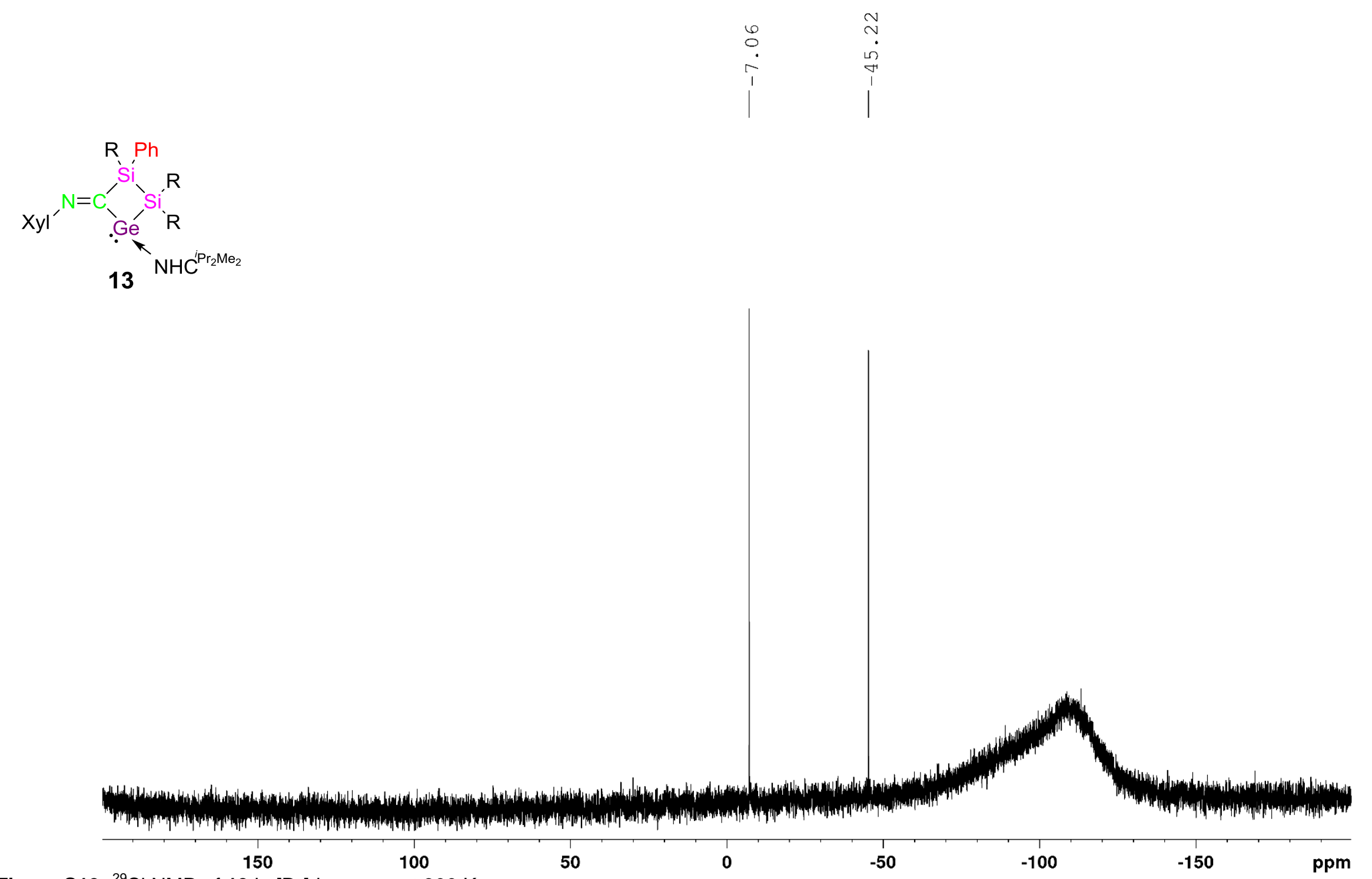

Figure S19: ${ }^{29} \mathrm{Si}$ NMR of 13 in $\left[\mathrm{D}_{6}\right]$-benzene at $300 \mathrm{~K}$. 


\section{UV/Vis spectra}

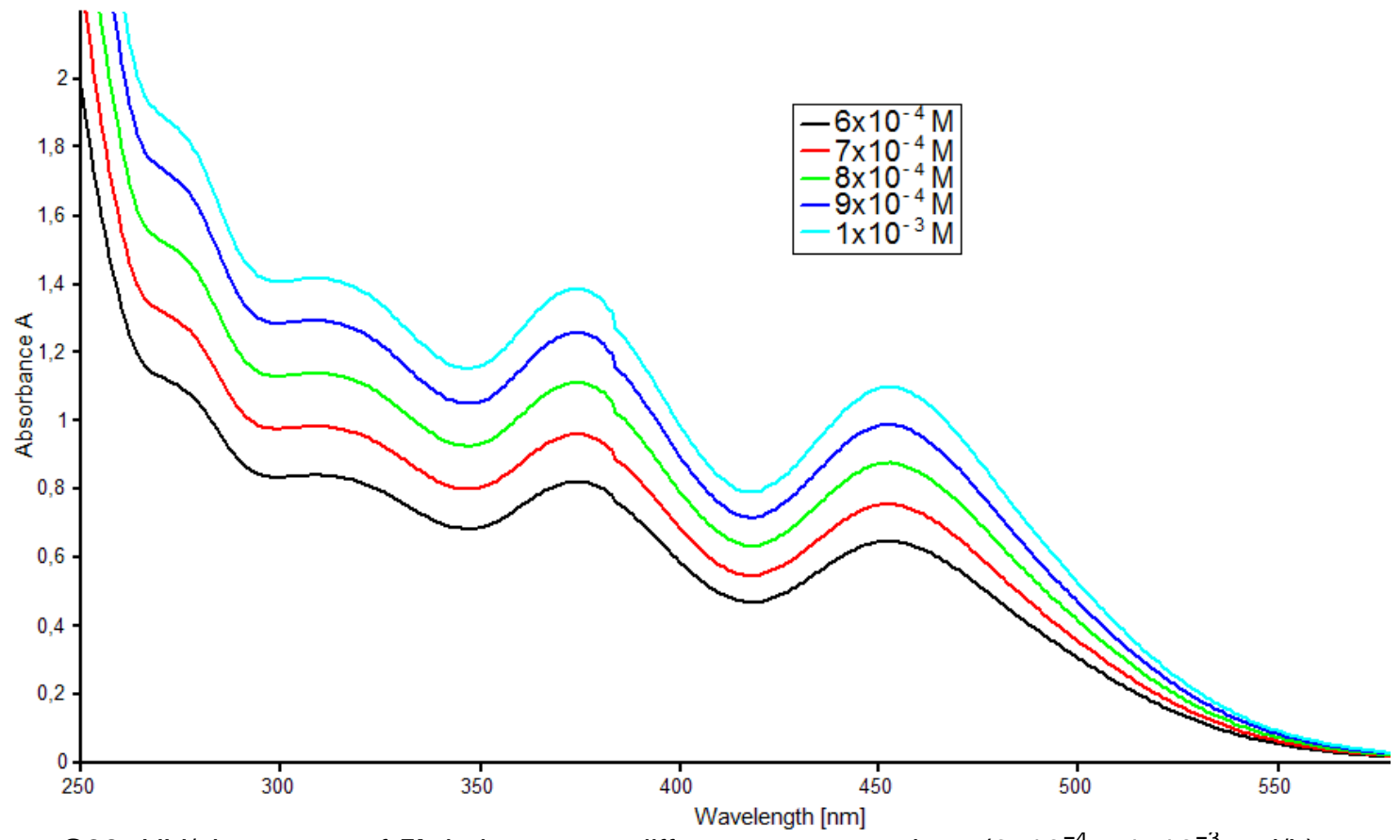

Figure S20: UV/vis spectra of $\mathbf{5 b}$ in hexane at different concentrations $\left(6 \times 10^{-4}-1 \times 10^{-3} \mathrm{~mol} / \mathrm{L}\right)$.

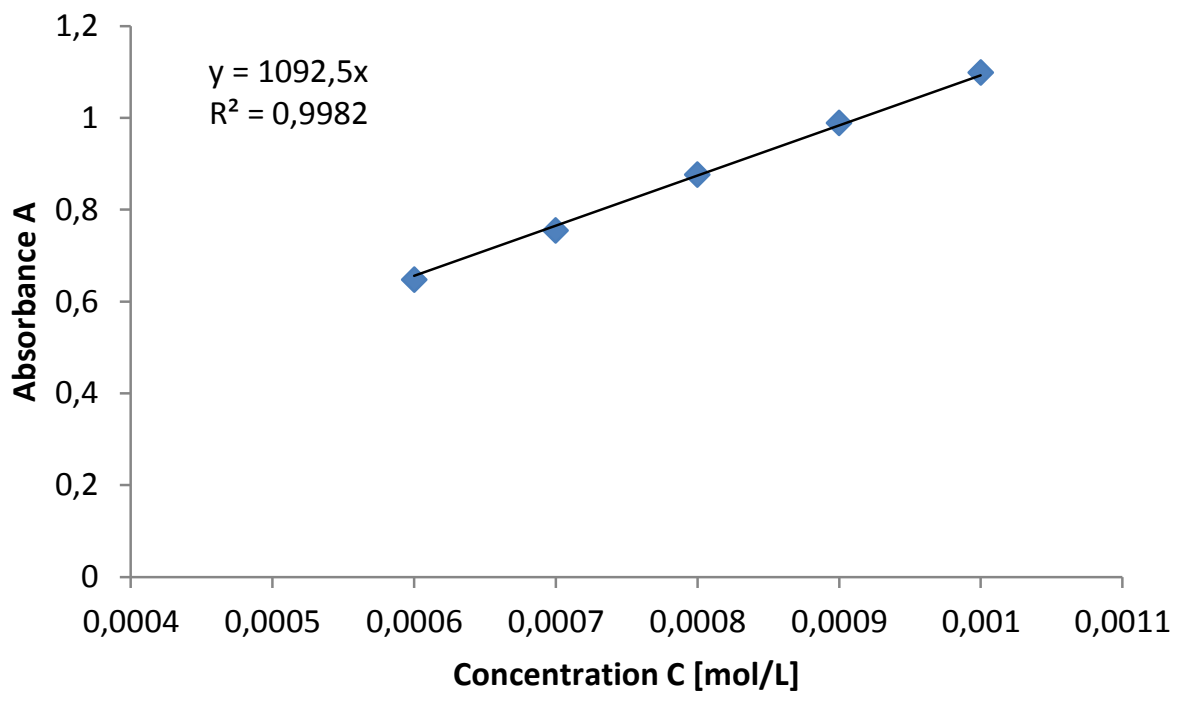

Figure S21: Linear regression of $\mathbf{5 b}$ at $452 \mathrm{~nm}$. 


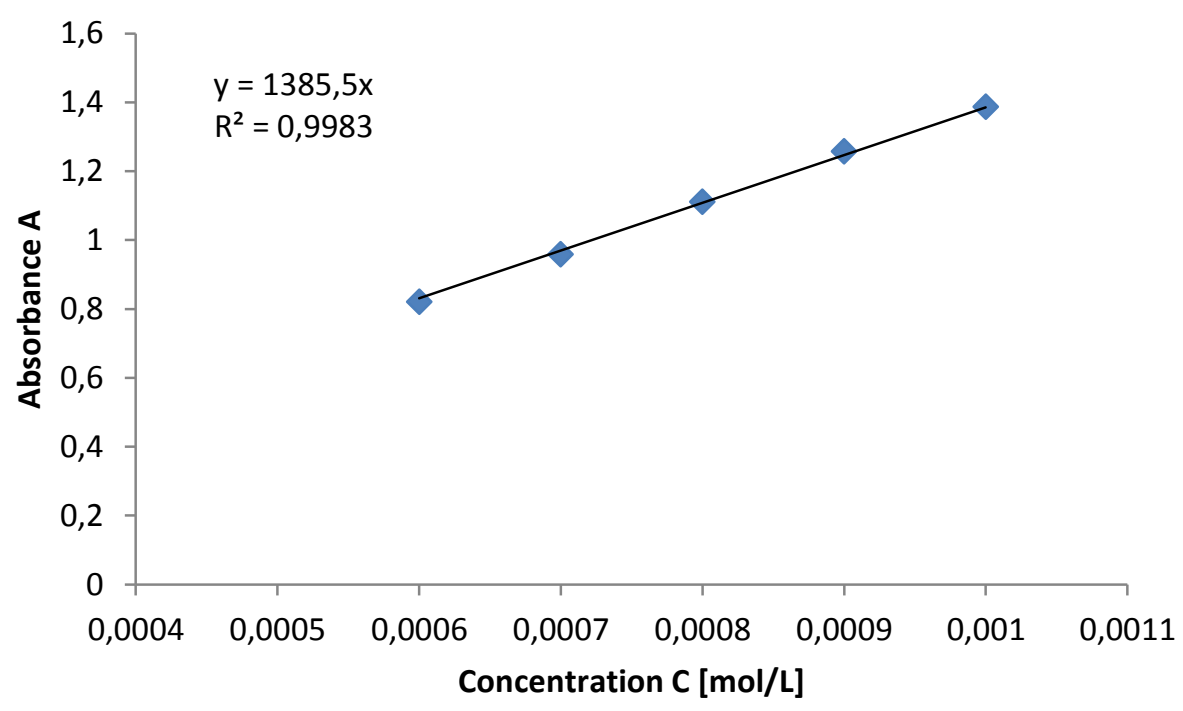

Figure S22: Linear regression of $\mathbf{5 b}$ at $373 \mathrm{~nm}$.

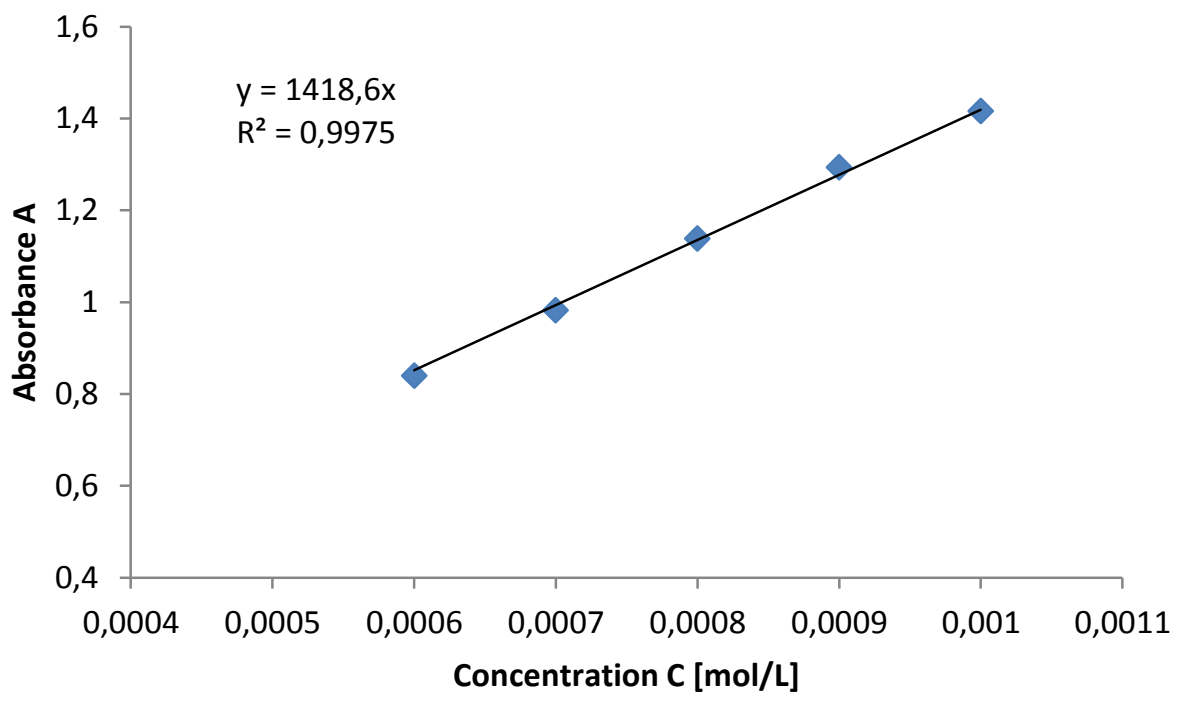

Figure S23: Linear regression of $\mathbf{5 b}$ at $308 \mathrm{~nm}$. 


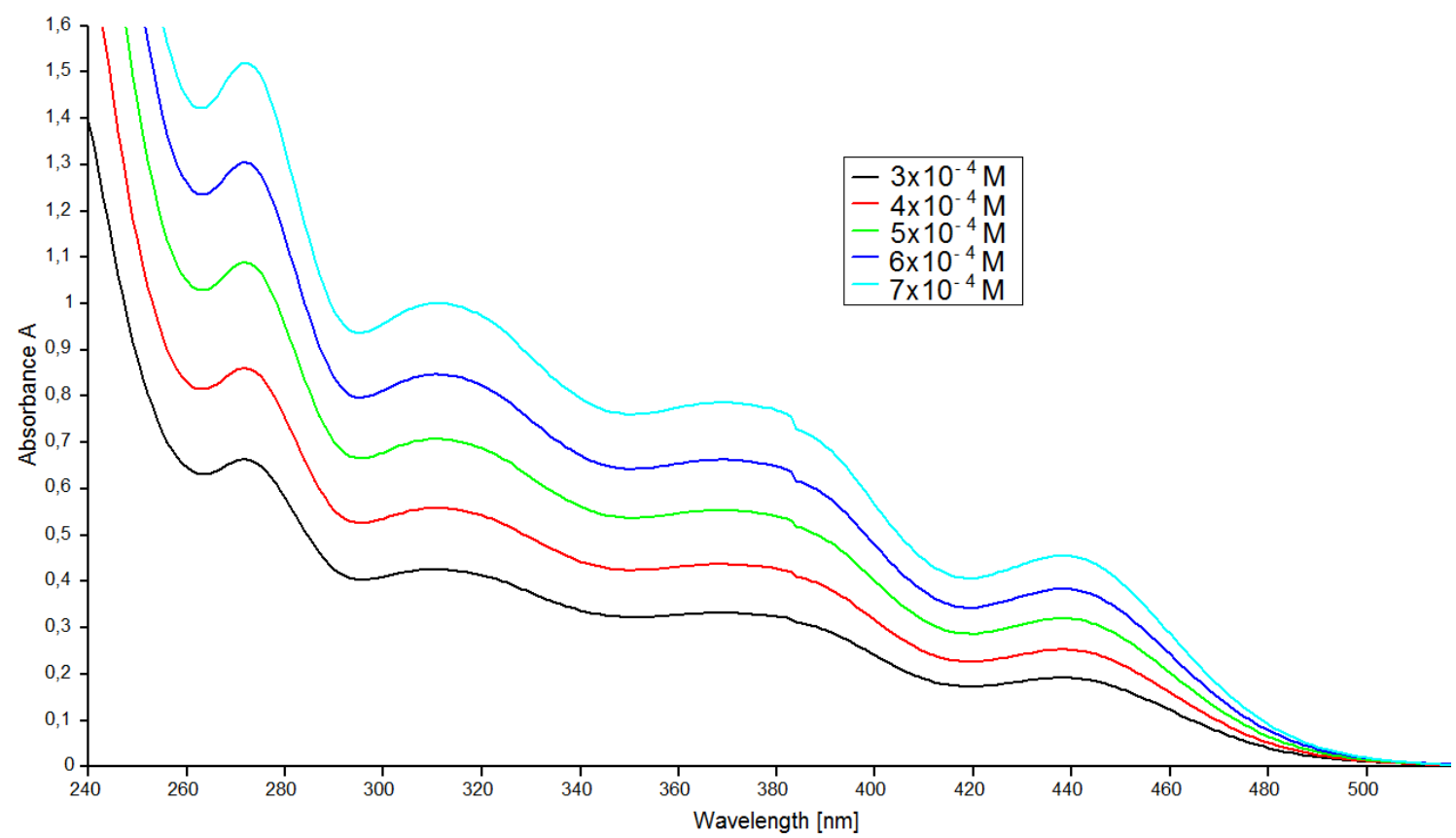

Figure S24: UV/vis spectra of $6 \mathrm{~b}$ in hexane at different concentrations $\left(3 \times 10^{-4}-7 \times 10^{-4} \mathrm{~mol} / \mathrm{L}\right)$.

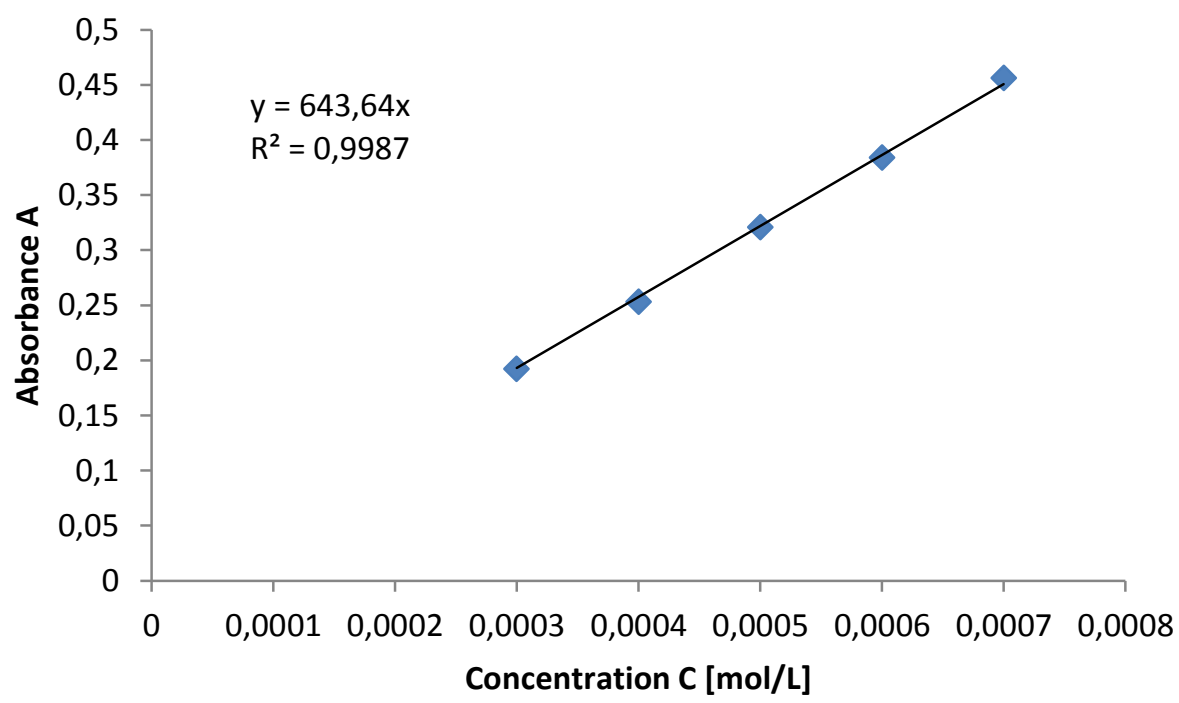

Figure S25: Linear regression of $6 \mathrm{~b}$ at $438 \mathrm{~nm}$. 


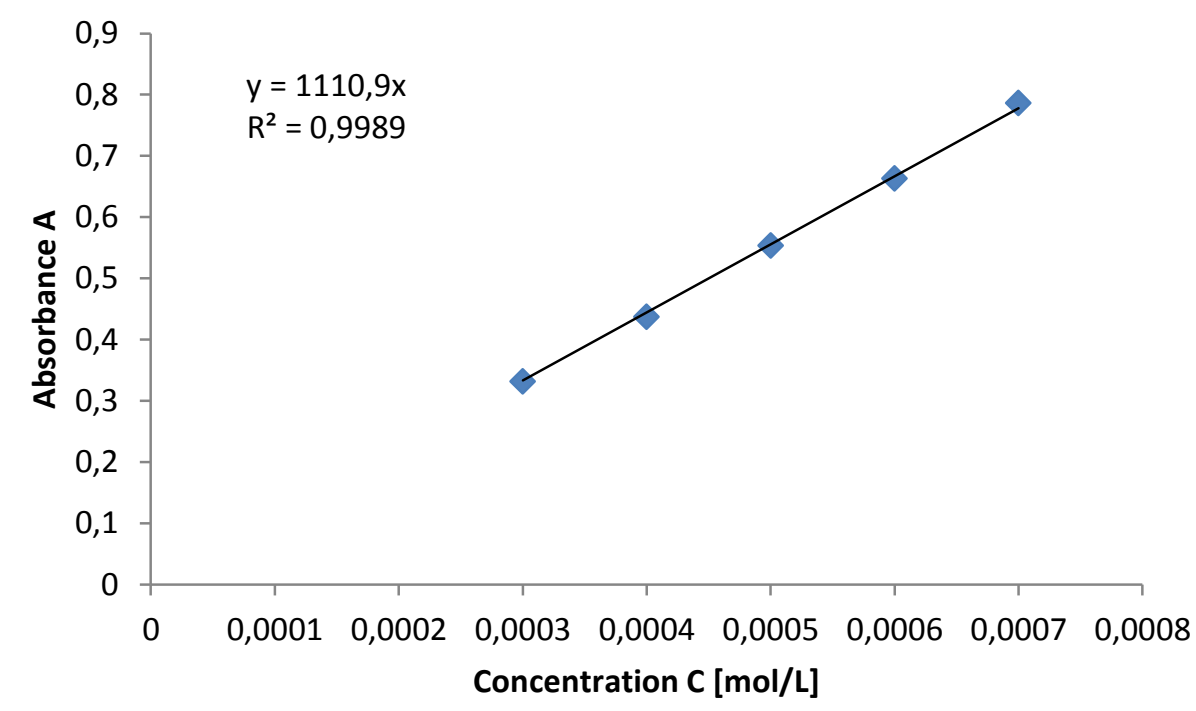

Figure S26: Linear regression of $\mathbf{6 b}$ at $370 \mathrm{~nm}$.

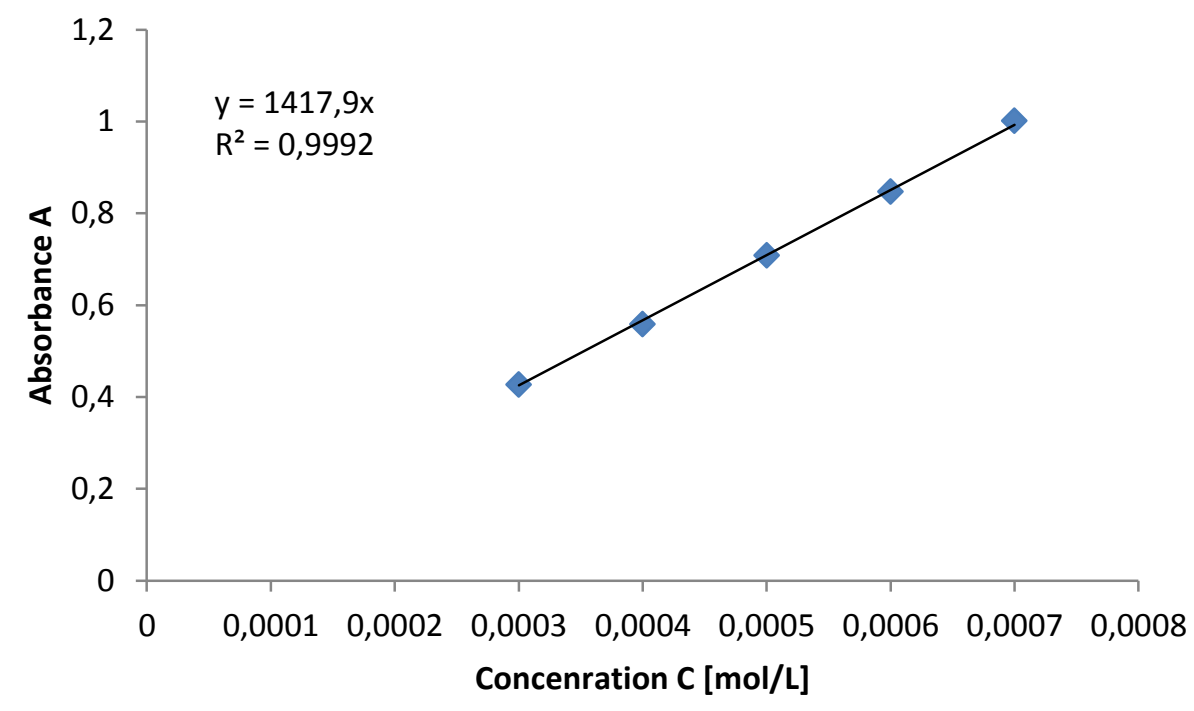

Figure S27: Linear regression of $6 \mathbf{b}$ at $311 \mathrm{~nm}$.

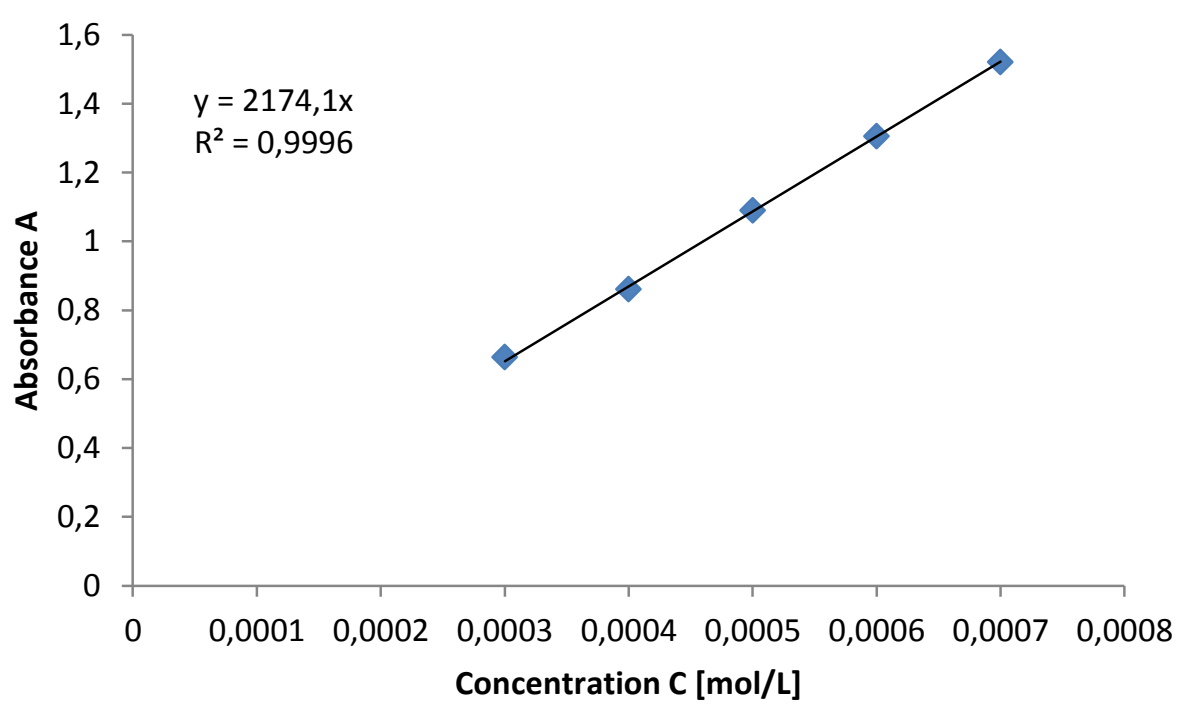

Figure S28: Linear regression of $6 \mathbf{b}$ at $272 \mathrm{~nm}$. 


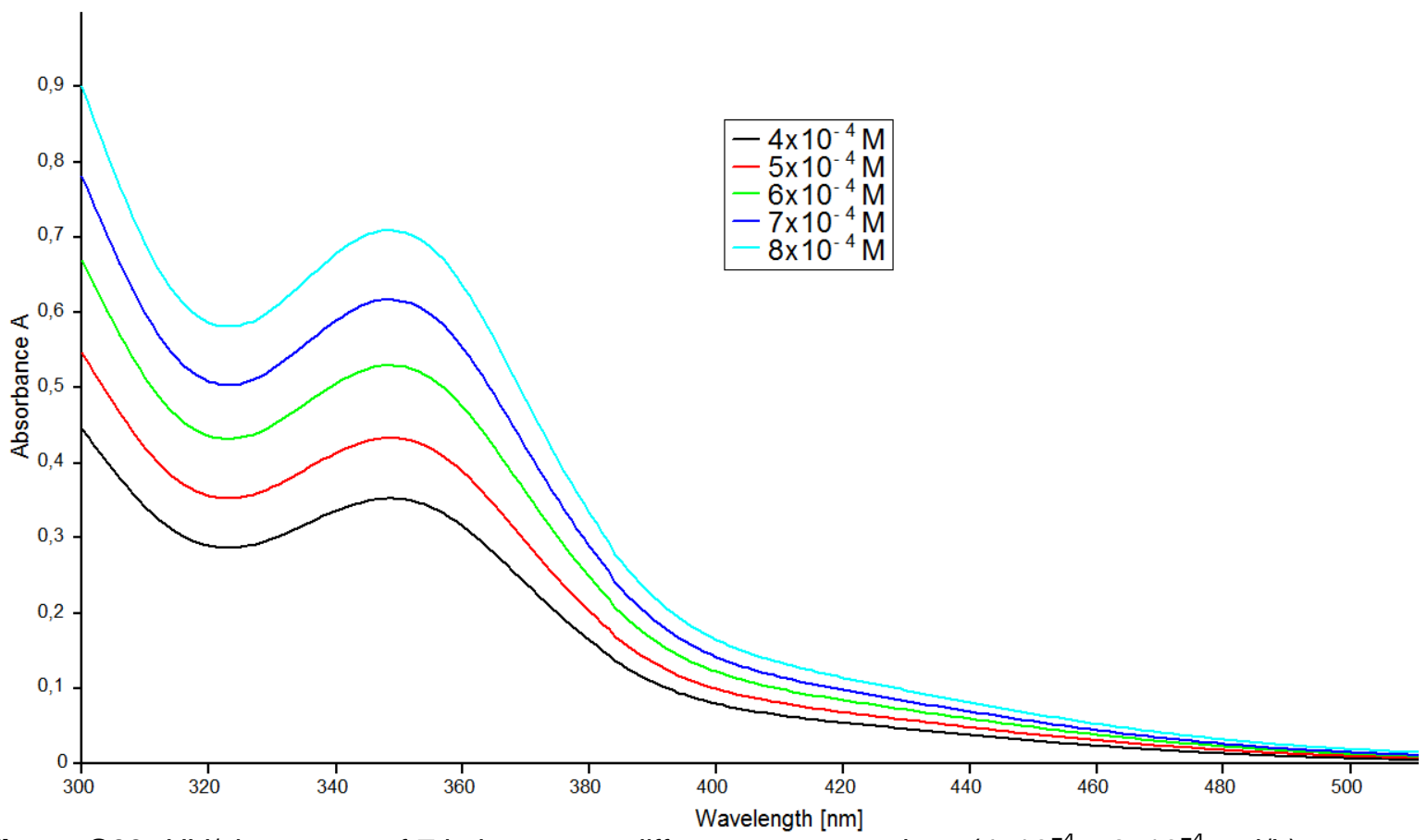

Figure S29: UV/vis spectra of 7 in hexane at different concentrations $\left(4 \times 10^{-4}-8 \times 10^{-4} \mathrm{~mol} / \mathrm{L}\right)$.

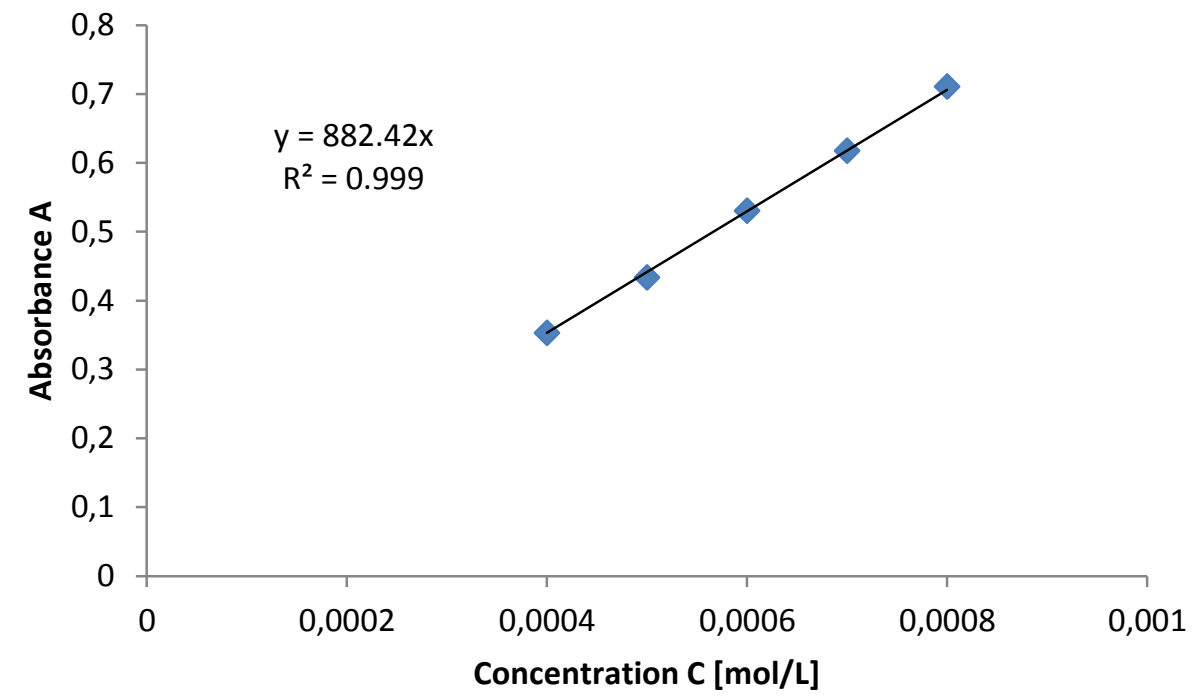

Figure S30: Linear regression of 7 at $348 \mathrm{~nm}$. 


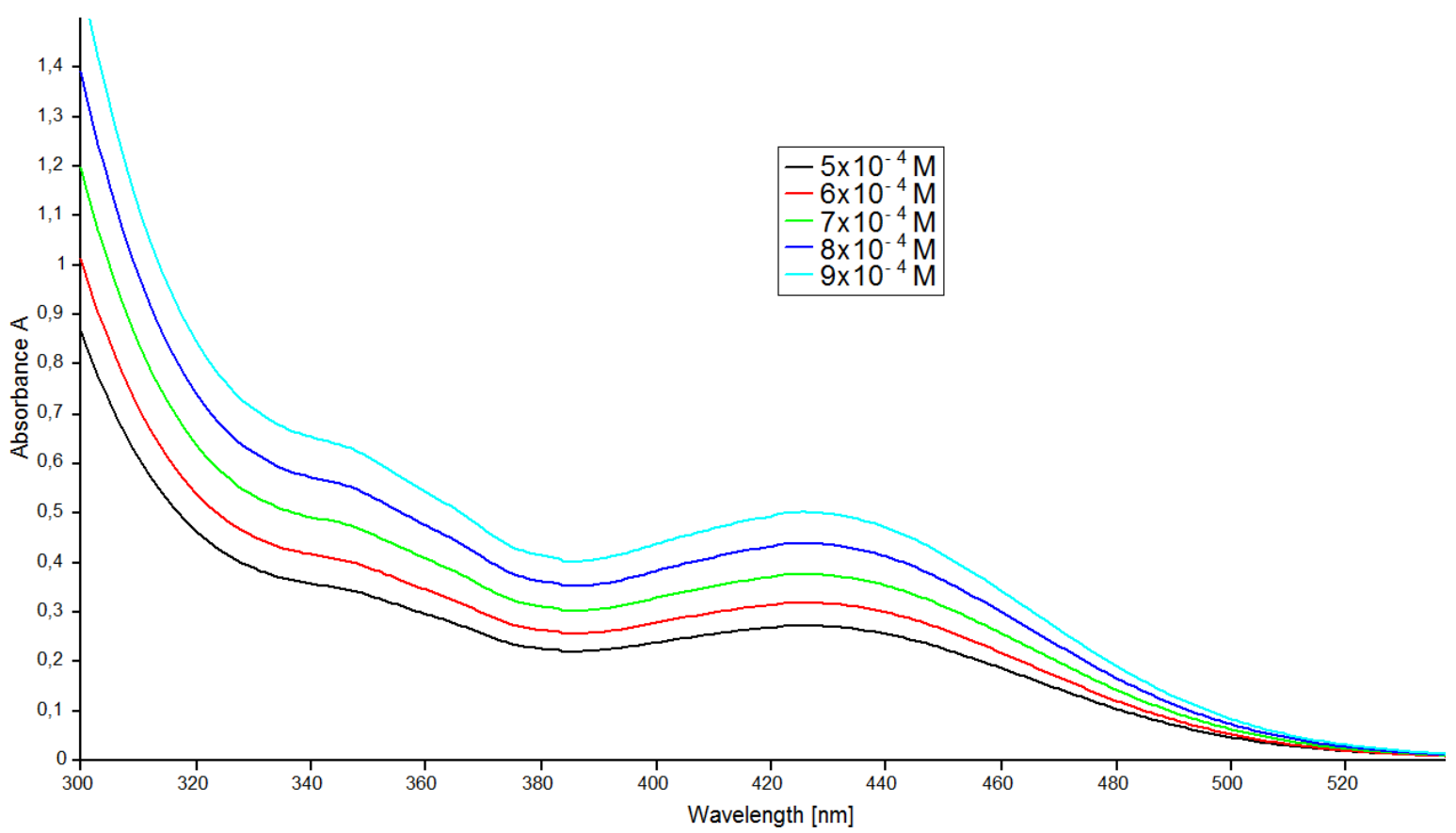

Figure S31: UV/vis spectra of 8 in hexane at different concentrations $\left(5 \times 10^{-4}-9 \times 10^{-4} \mathrm{~mol} / \mathrm{L}\right)$.



Figure S32: Linear regression of 8 at $425 \mathrm{~nm}$. 


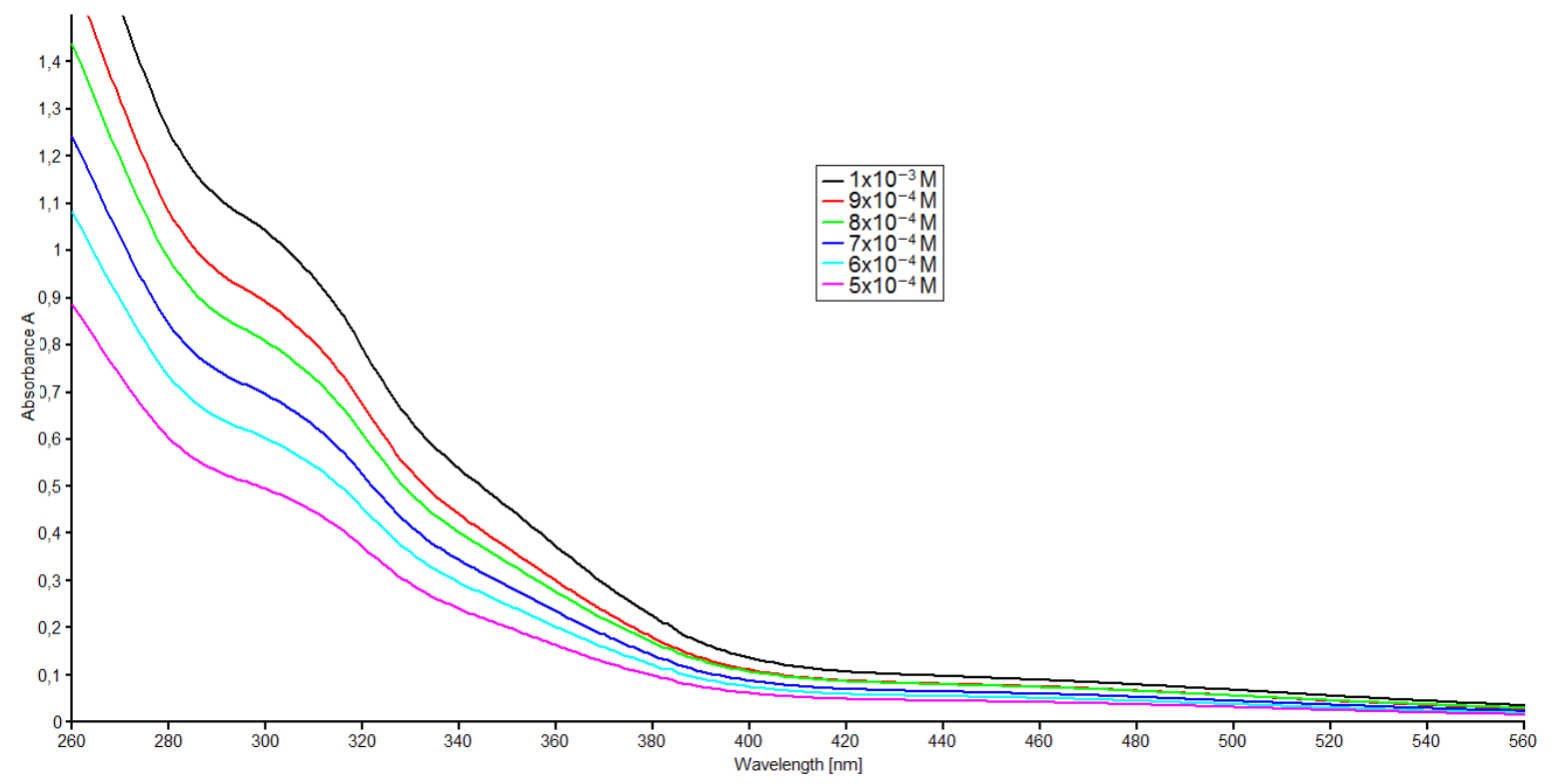

Figure S33: UV/vis spectra of 12 in hexane at different concentrations $\left(5 \times 10^{-4}-1 \times 10^{-3} \mathrm{~mol} / \mathrm{L}\right)$.

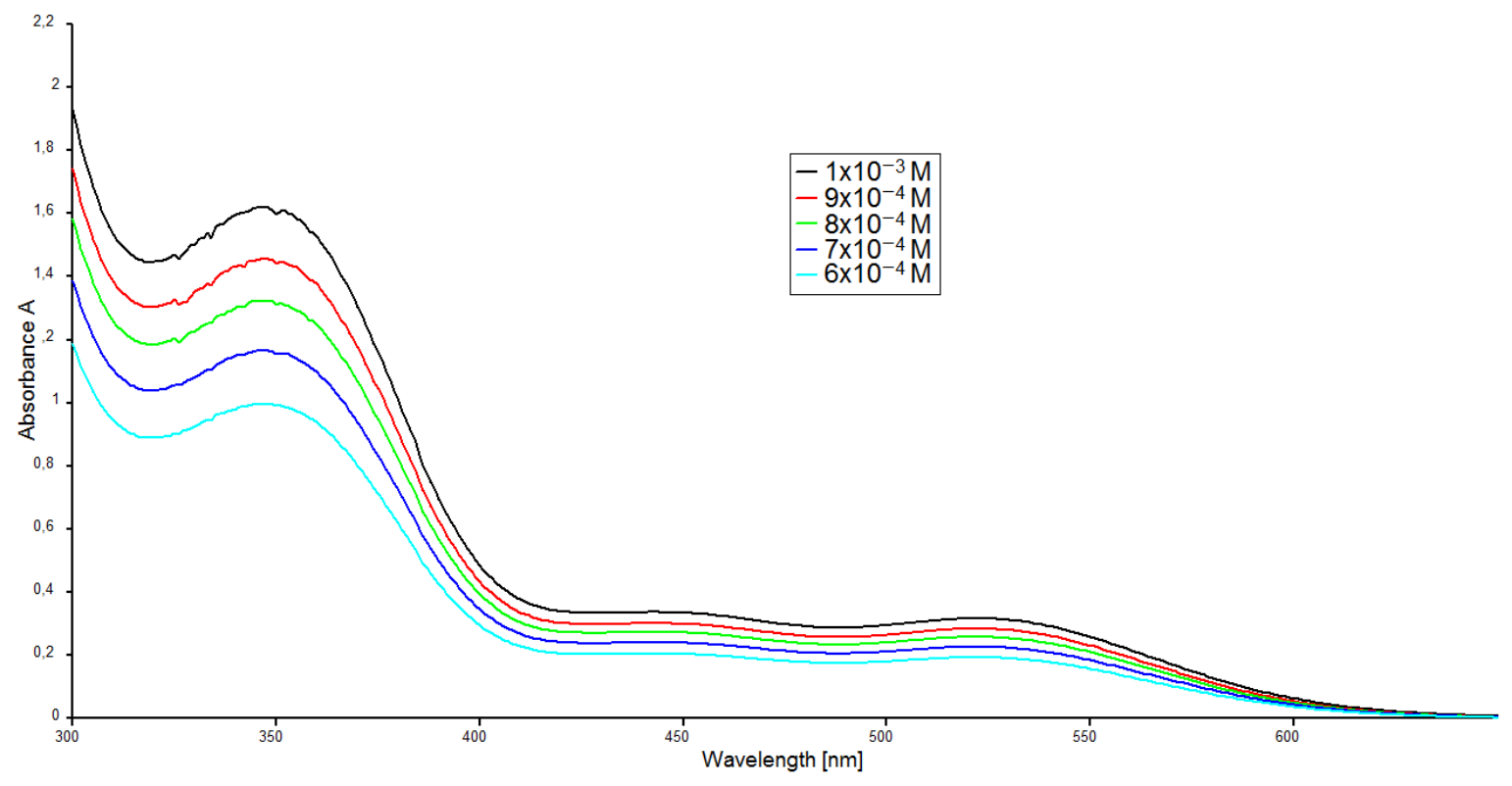

Figure S34: UV/vis spectra of 13 in hexane at different concentrations $\left(6 \times 10^{-4}-1 \times 10^{-3} \mathrm{~mol} / \mathrm{L}\right)$. 


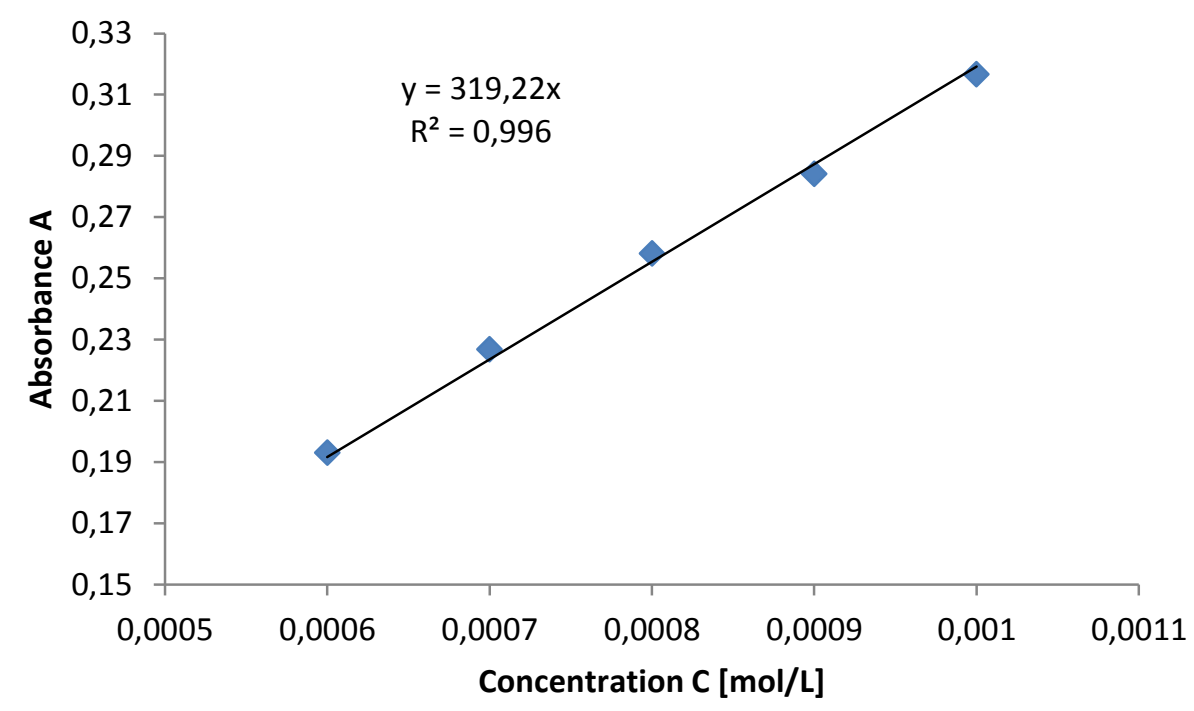

Figure S35: Linear regression of $\mathbf{1 3}$ at $522 \mathrm{~nm}$.

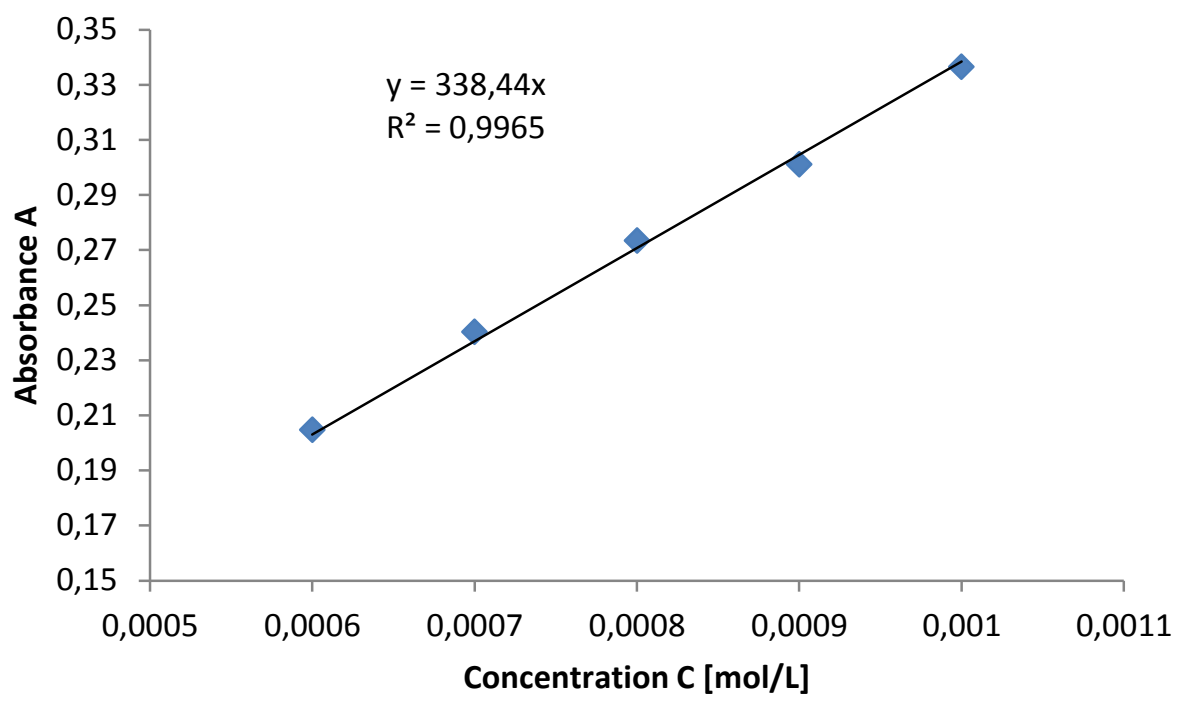

Figure S36: Linear regression of 13 at $442 \mathrm{~nm}$. 


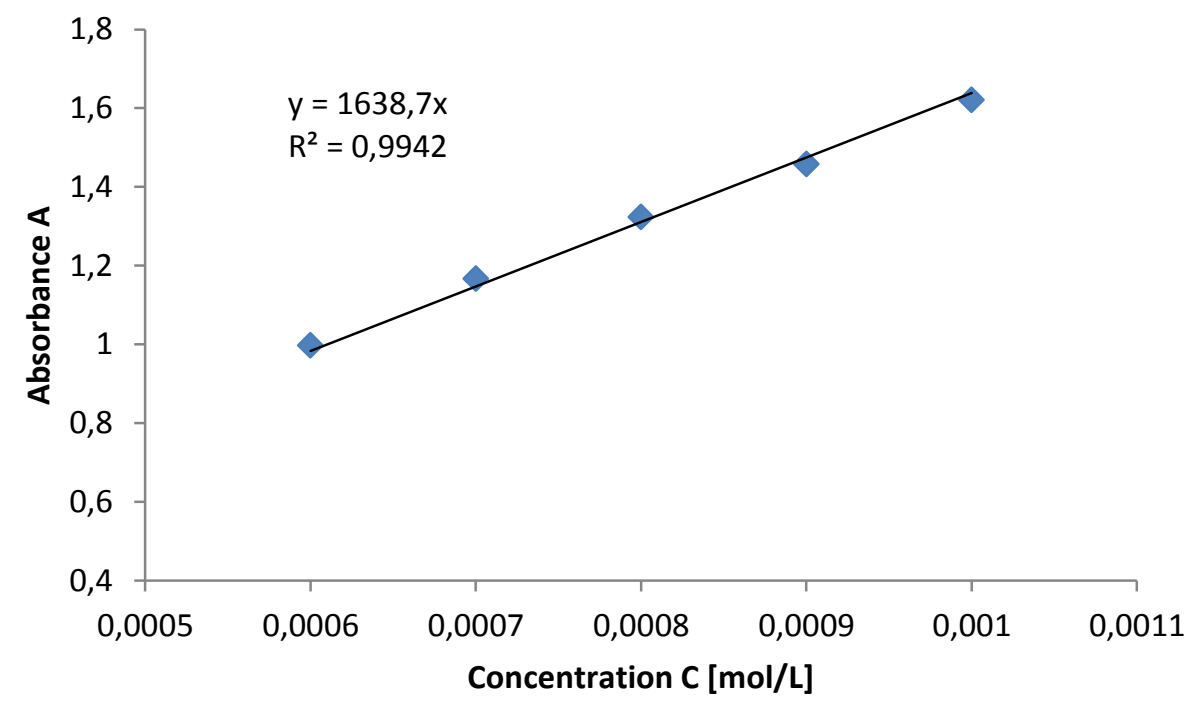

Figure S37: Linear regression of $\mathbf{1 3}$ at $347 \mathrm{~nm}$. 


\section{Computational details}

All theoretical calculations, in this paper are carried out using the Gaussian09 suite of programs. ${ }^{1}$ The proposed mechanisms and intermediates for the formation of 7-Me, 8-Me, and 12-Ph were calculated using simplified model system with methyl groups instead of Tip, $\mathrm{Ph}$, and ${ }^{i} \mathrm{Pr}$ groups (Figure S42 to S52). The B3LYP/6-31G(d,p) was chosen as level of theory due to dramatic raise in the computational costs with higher level of theories. Frequency analyses were performed to determine the character of optimized structures as minima or transition states. The relative Gibbs free energies are given in kcal $\mathrm{mol}^{-1}$. The chemical reaction channels have been checked by the intrinsic reaction coordinate (IRC) method to verify the energy profiles at the B3LYP/6-31G(d,p) level of theory for the processes from transition states to intermediates, by using the second-order Gonzalez-Schlegel method. ${ }^{2,3}$ The electronic absorption spectrum for 7-Me, 8-Me, 12-Me, and 13-Me was predicted using the optimized geometry with the time-dependent density functional theory (TD-DFT) method in heptane solution at $\mathrm{B} 3 \mathrm{LYP} / 6-31+\mathrm{G}(\mathrm{d}, \mathrm{p})$ level of theory. The GaussView 5.0 program was employed for visualization of the final geometries of the optimized structures. ${ }^{4}$ 
Figure S38: Calculated UV/vis spectrum of 7-Me in hexane at B3LYP/6-31+G(d,p) level of theory (solvent $=$ heptane).

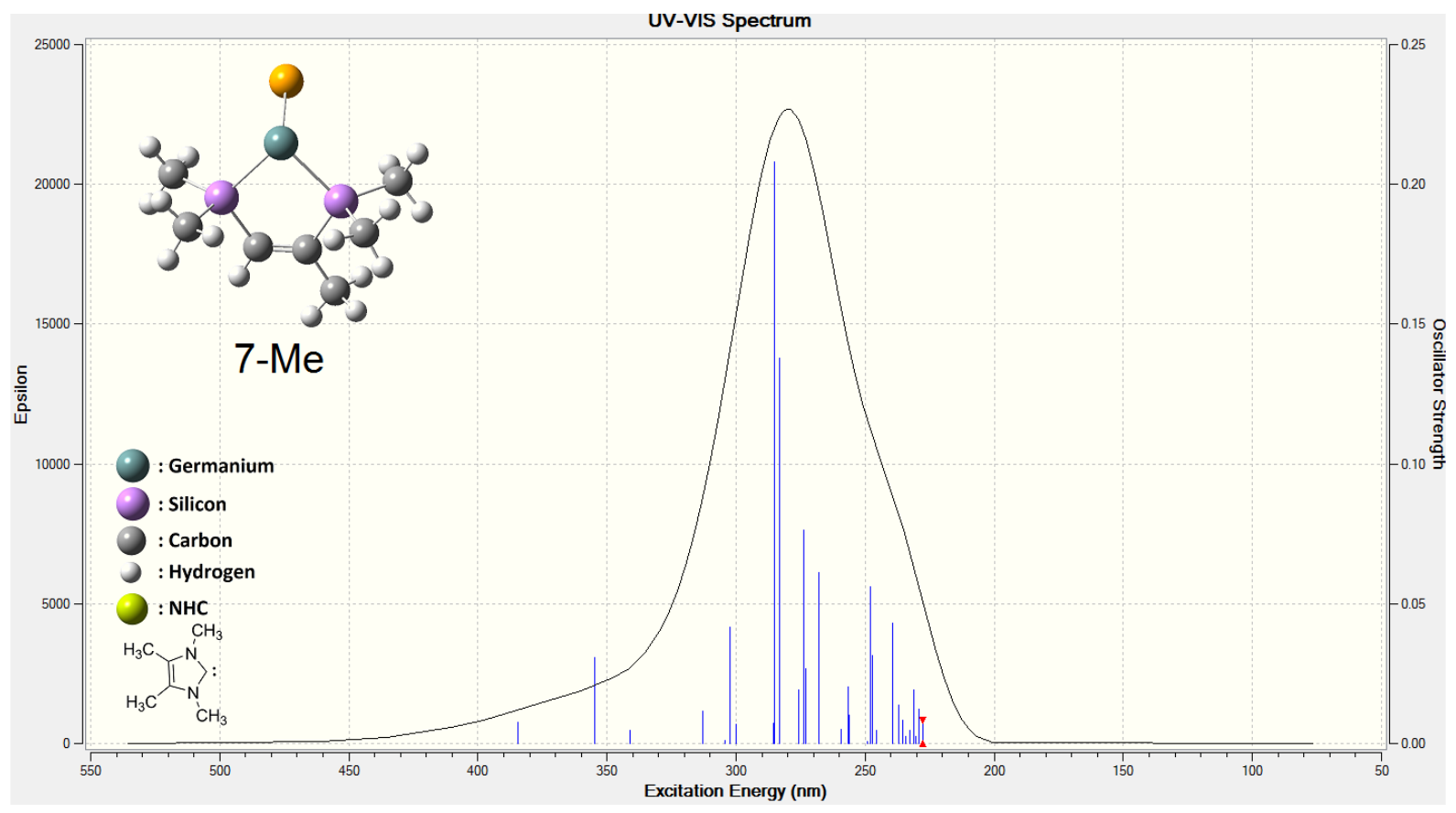

Figure S39: Calculated UV/vis spectrum of 8-Me in hexane at B3LYP/6-31+G(d,p) level of theory (solvent $=$ heptane).

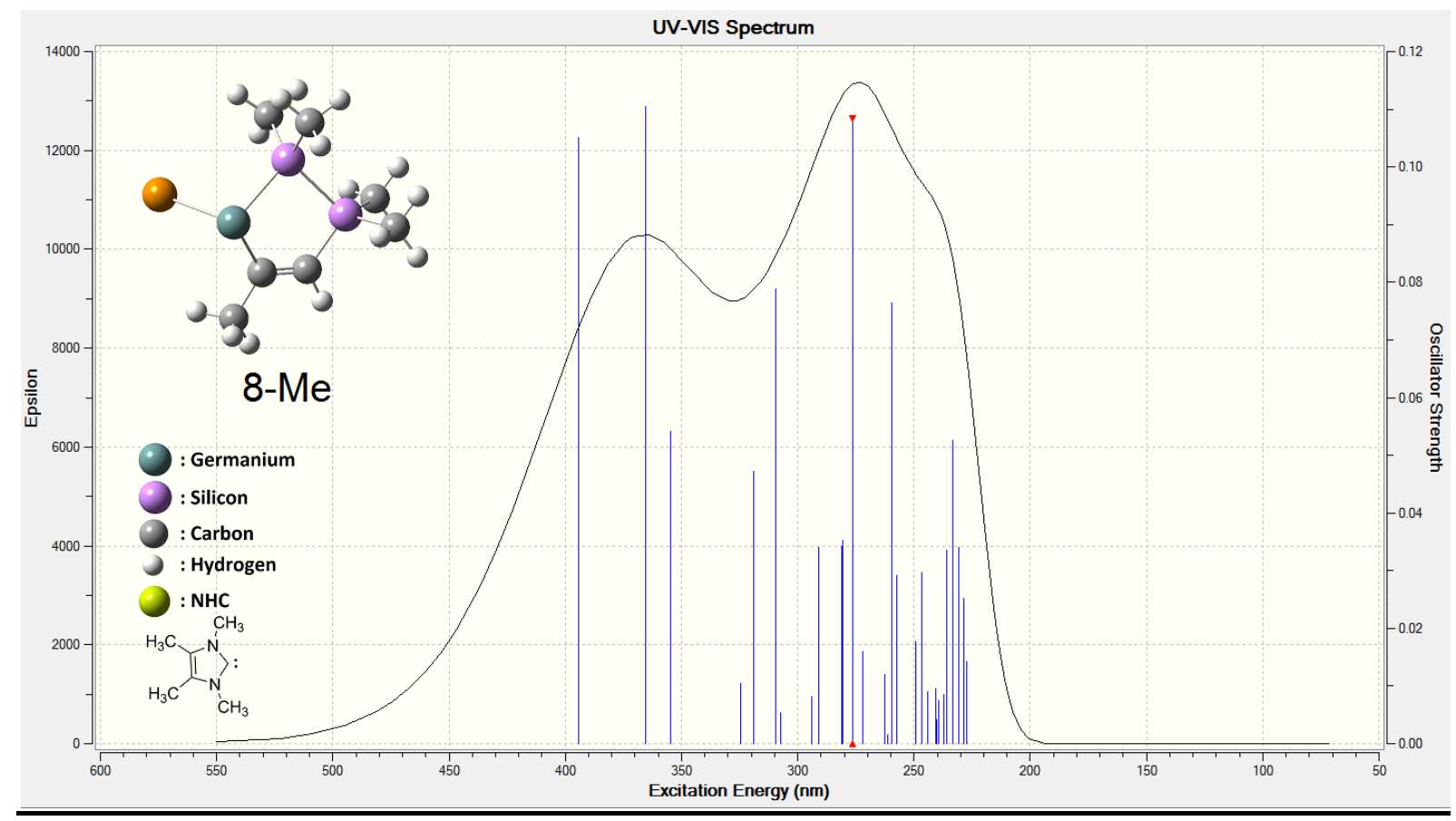


Figure S40: Calculated UV/vis spectrum of 12-Me in hexane at B3LYP/6-31+G(d,p) level of theory (solvent $=$ heptane).

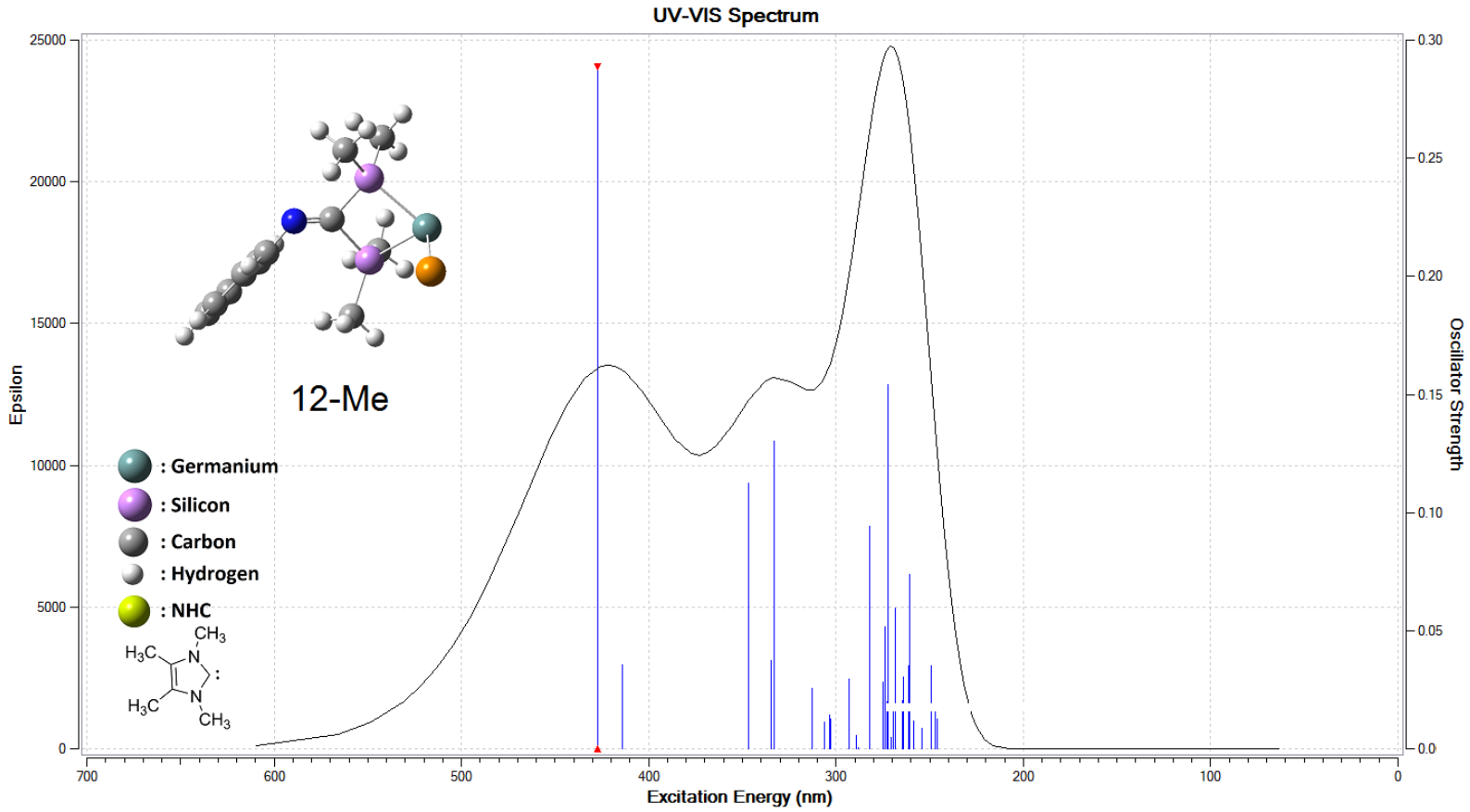

Figure S41: Calculated UV/vis spectrum of 13-Me in hexane at B3LYP/6-31+G(d,p) level of theory (solvent $=$ heptane) .

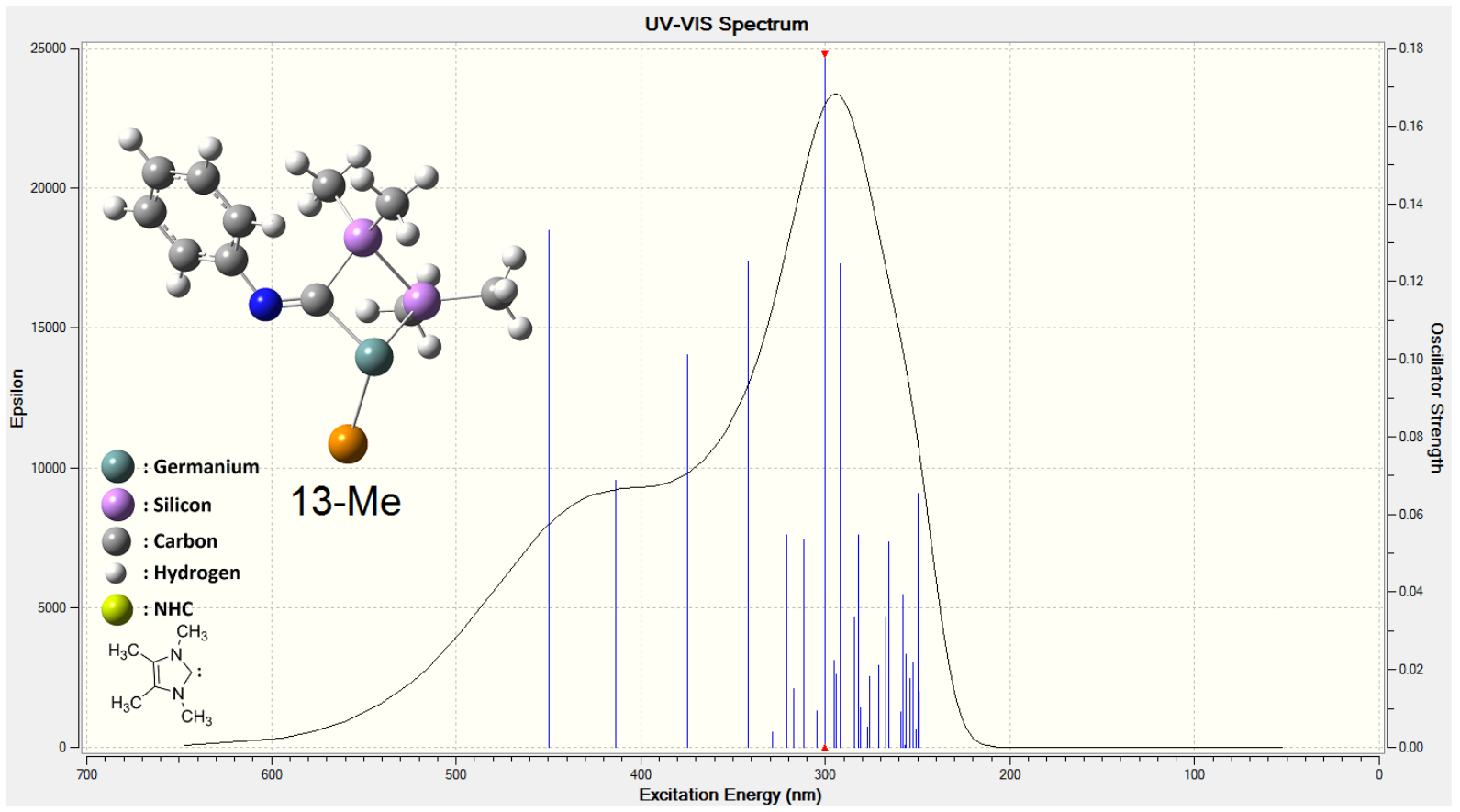


Figure S42. First proposed mechanism and intermediates for the formation of 9-Me from $\mathbf{5 b}$-Me+Ac using simplified model system with methyl groups instead of Tip, $\mathrm{Ph}$, and ${ }^{\prime} \mathrm{Pr}$ groups at B3LYP/6-31G(d,p) level of theory, respectively. $\Delta \mathrm{G}$ energy values at $298 \mathrm{~K}$ are given in kcal mol ${ }^{-1}$.

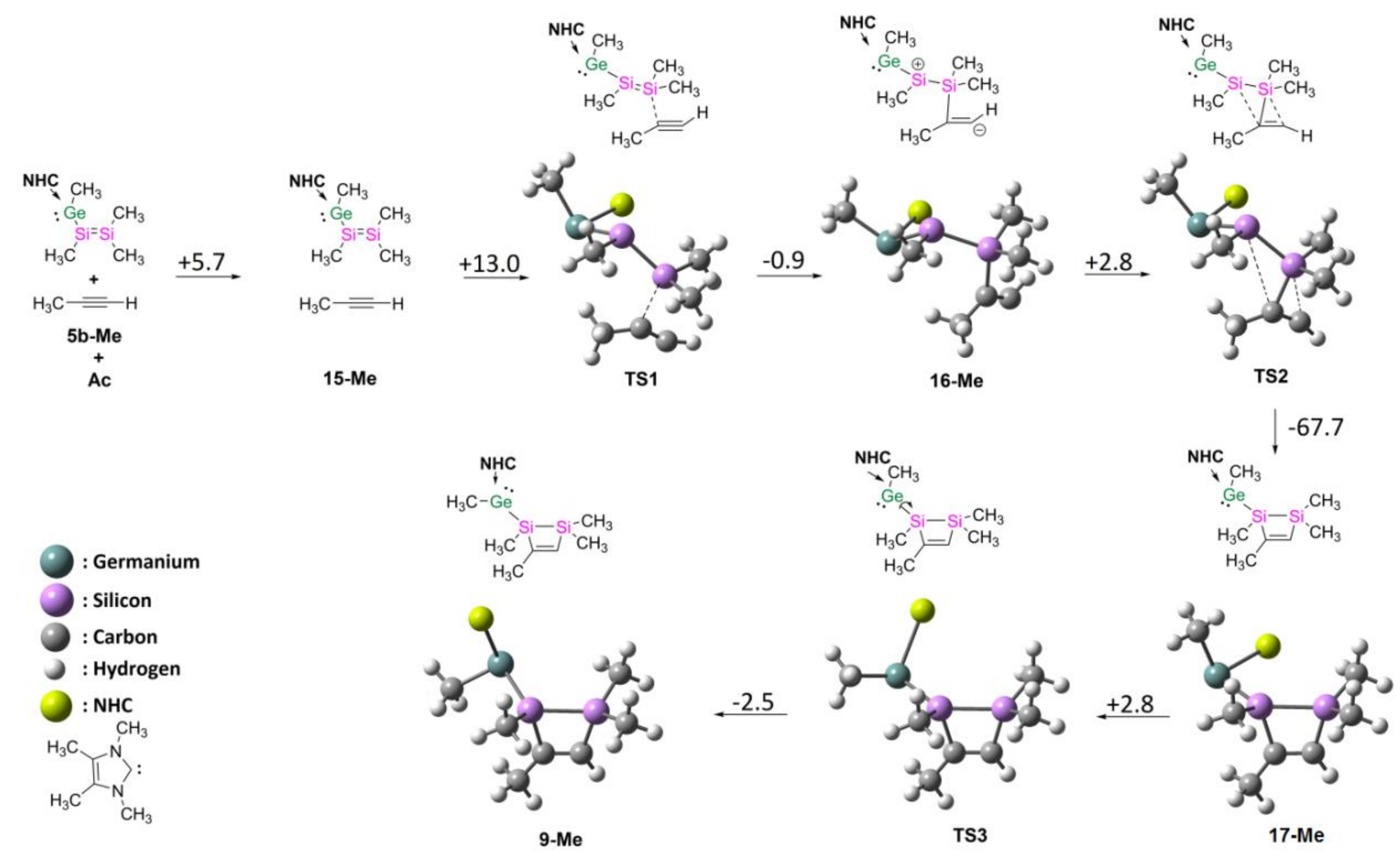


Figure S43. Proposed mechanism and intermediates for the formation of 7-Me from NHC coordinated system using simplified model system with methyl groups instead of $\mathrm{Tip}, \mathrm{Ph}$, and ${ }^{\mathrm{P}} \mathrm{Pr}$ groups at B3LYP/6-31G $(d, p)$ level of theory, respectively. $\Delta G$ energy values at $298 \mathrm{~K}$ are given in $\mathrm{kcal}^{\mathrm{mol}}{ }^{-1}$.

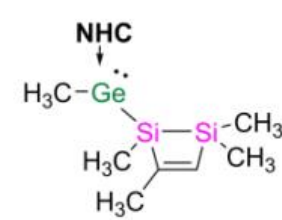

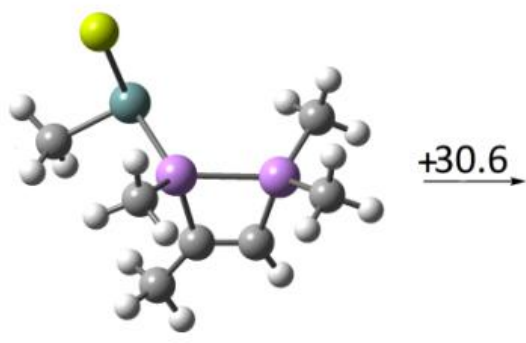

9-Me<smiles>CN[14C]1(C)[Si](C)(C)C=C(C)[Si]1(C)C</smiles>

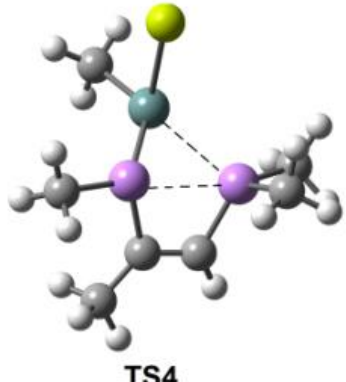

TS4<smiles>CC1=C[Si](C)(C)[Ge]23C(C)(C)[C@]2(C)[C@]13C</smiles>

$-48.2$
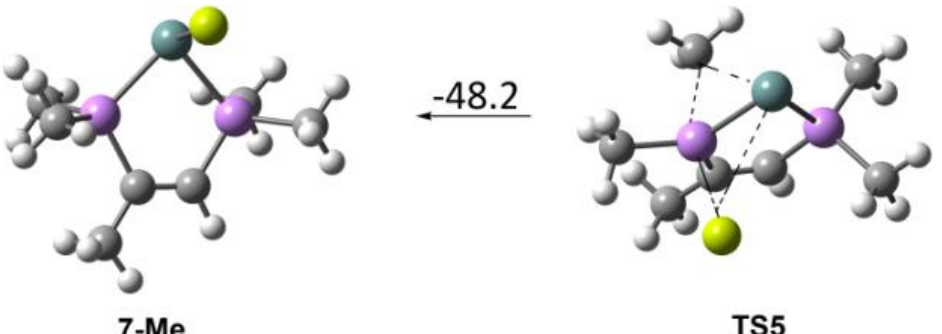

$+31.5$<smiles>CN[14C@@]1(C)[Si](C)(C)C=C(C)[Si]1(C)C</smiles>

$-32.7$

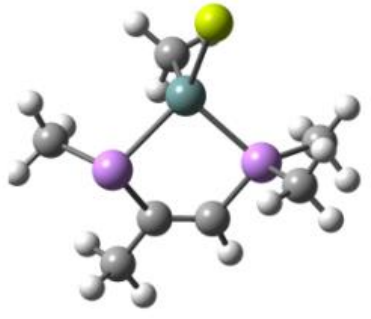

18-Me

$-4.0$<smiles>CC1=C[Si](C)(C)[Ge](C)([O])[Ge]1(C)C=N</smiles>

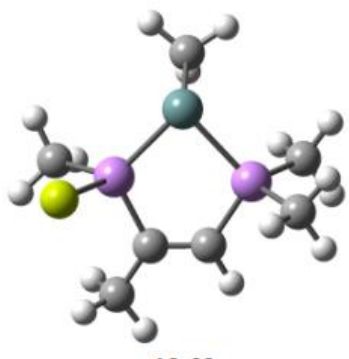

19-Me 
Figure S44. Proposed mechanism and intermediates for the formation of 7-Me from NHC free system using simplified model system with methyl groups instead of Tip, Ph, and ${ }^{i} \mathrm{Pr}$ groups at B3LYP/6-31G(d,p) level of theory, respectively. $\Delta \mathrm{G}$ energy values at $298 \mathrm{~K}$ are given in kcal mol${ }^{-1}$.
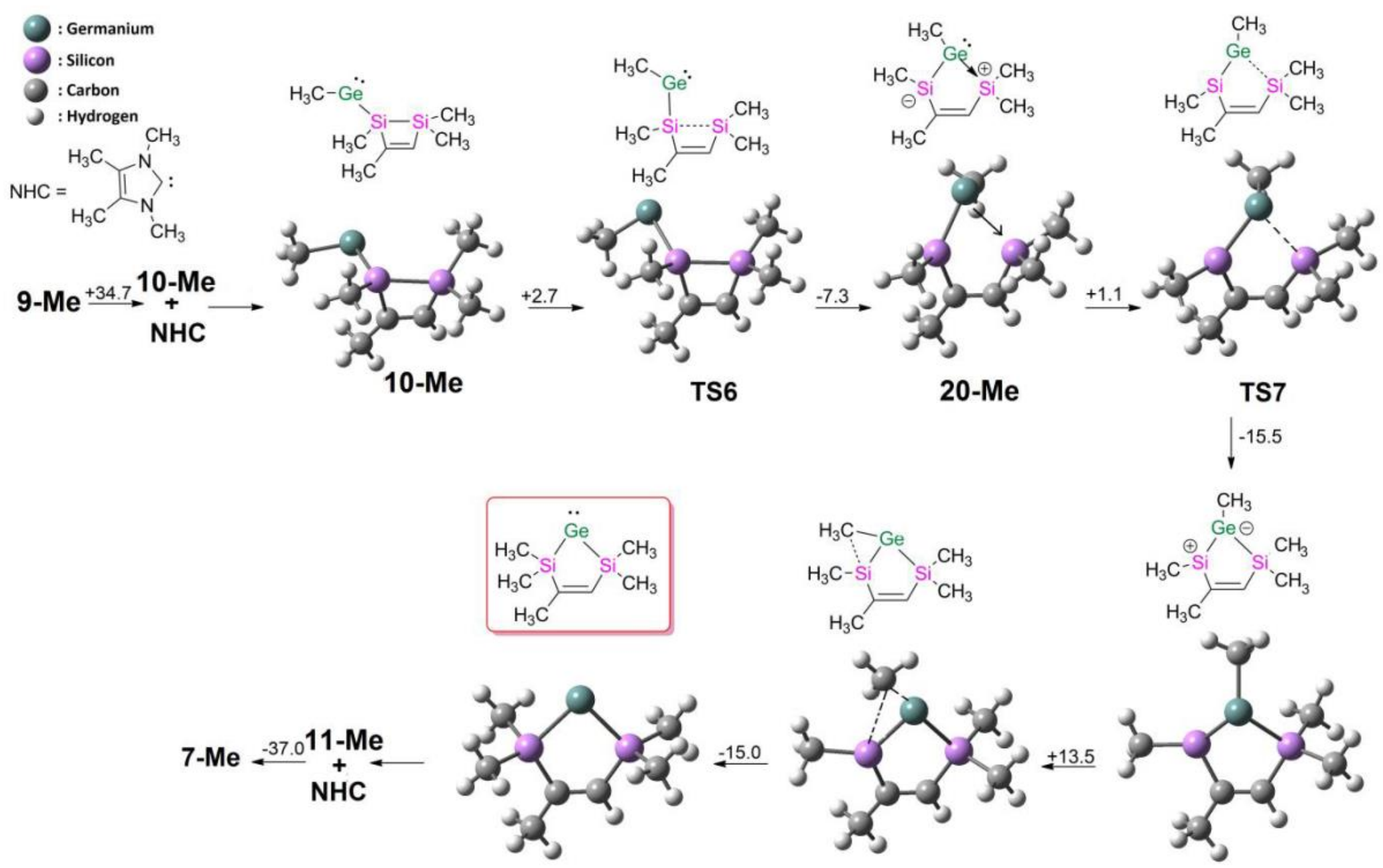

11-Me
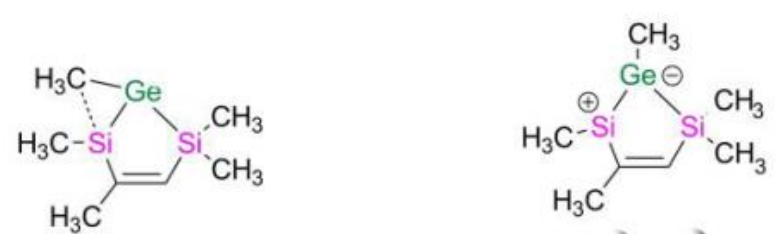

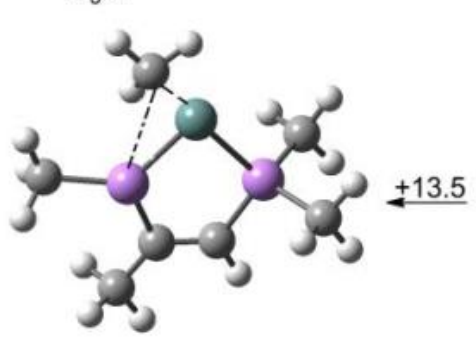

TS8

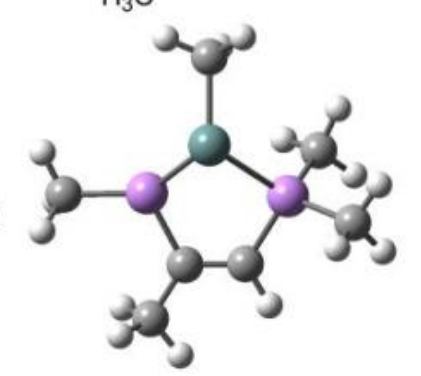

21-Me 
Figure S45. Alternative mechanism and intermediates for the formation of 9-Me from $\mathbf{5 b - M e + A c ~ u s i n g ~}$ simplified model system with methyl groups instead of Tip, $\mathrm{Ph}$, and ${ }^{i} \mathrm{Pr}$ groups at B3LYP/6-31G(d,p)

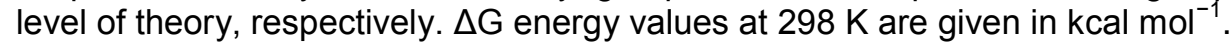

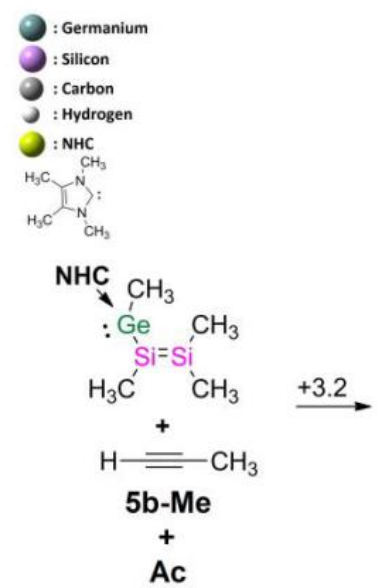

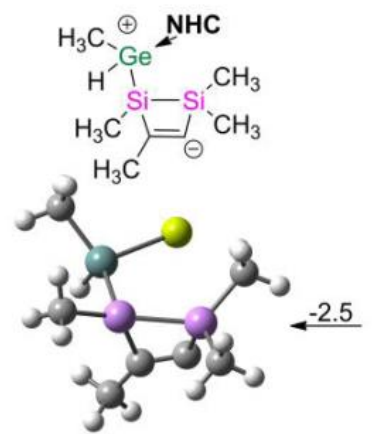

25-Me

$+6.2$<smiles>CC1=C(C)[Si](C)(C)[Si](C)(C)O1</smiles>

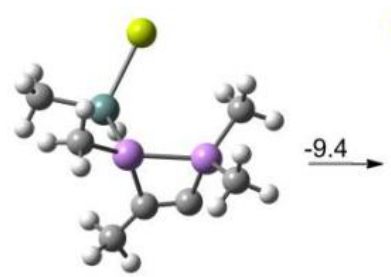

TS12

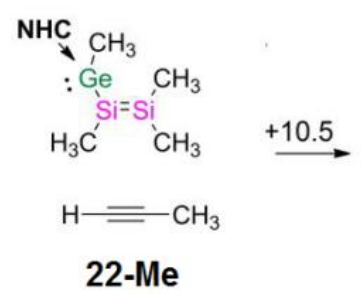

$\mathrm{H}_{3} \mathrm{C} \stackrel{\oplus}{\oplus} \mathrm{NHC}$
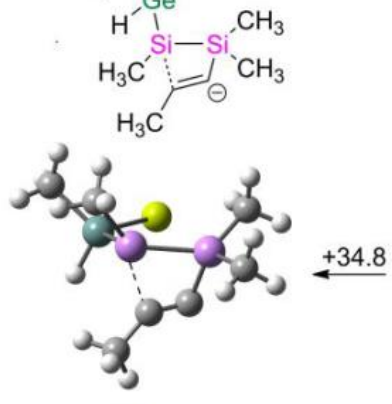

TS11
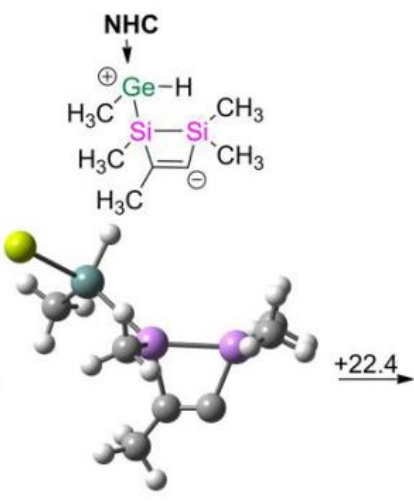

26-Me<smiles>C/C=C\[Si](C)(C)[Si](C)(C)C</smiles><smiles>CC#C[Si](C)(C)[Si](C)(C)O[Mg]</smiles>

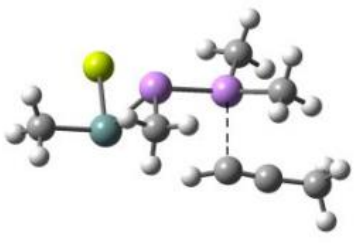

TS9
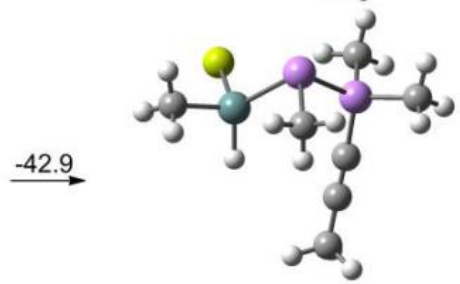

23-Me

$\downarrow+18.7$

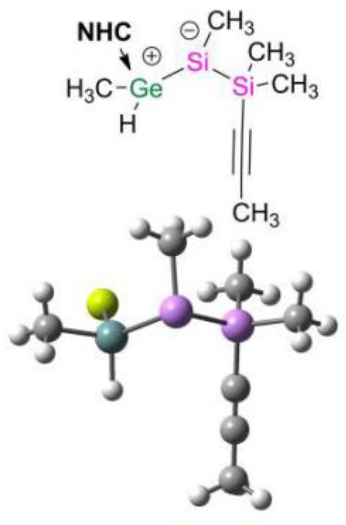

24-Me

$-17.2$
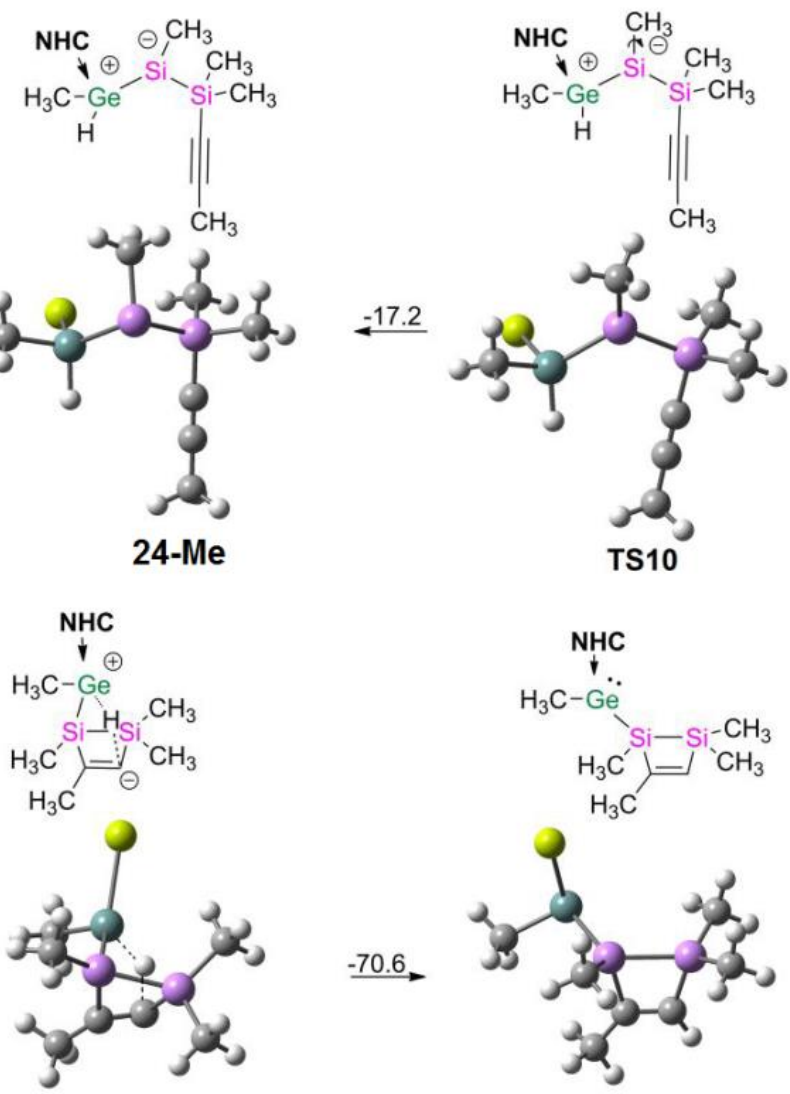

TS13

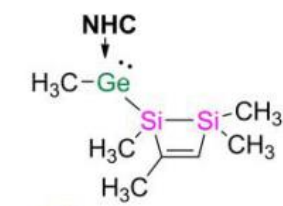

$-70.6$

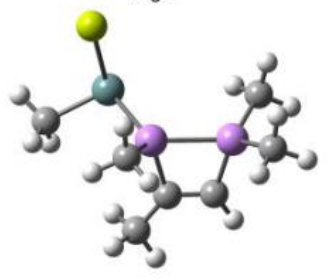

9-Me 
Figure S46. Unfavored mechanism due to high energy barriers and intermediates for the formation of 8-Me from [2+3] addition of 5b-Me to Ac using simplified model system with methyl groups instead of Tip, $\mathrm{Ph}$, and ${ }^{\mathrm{P}} \mathrm{Pr}$ groups at B3LYP/6-31G(d,p) level of theory, respectively. $\Delta \mathrm{G}$ energy values at $298 \mathrm{~K}$ are given in $\mathrm{kcal} \mathrm{mol}{ }^{-1}$.

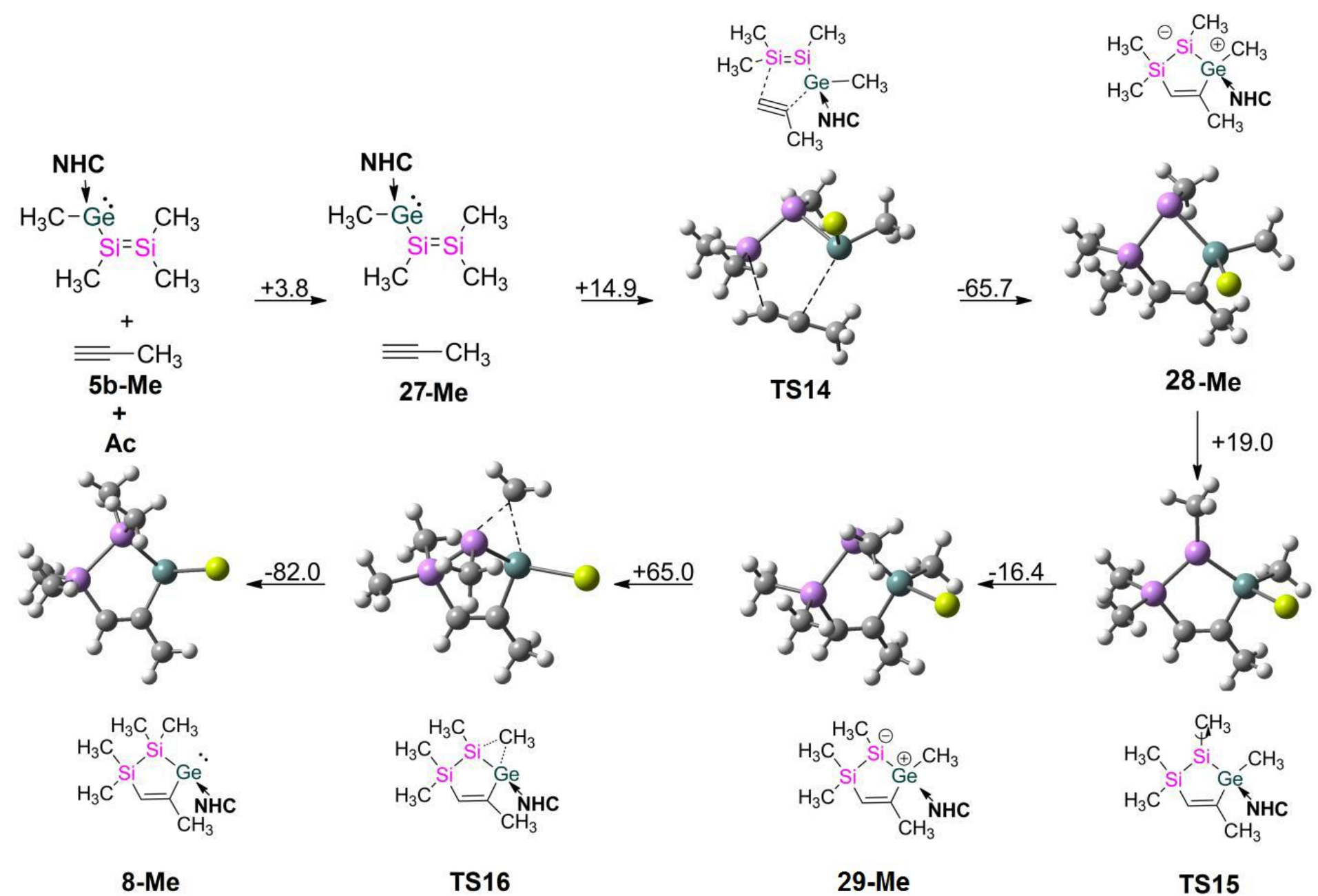


Figure S47. Proposed mechanism and intermediates for the formation of 8-Me from insertion of Ac to Si-Ge single bond of $\mathbf{6 b}-\mathrm{Me}$ using simplified model system with methyl groups instead of Tip, $\mathrm{Ph}$, and ${ }^{i} \operatorname{Pr}$ groups at B3LYP/6-31G(d,p) level of theory, respectively. $\Delta \mathrm{G}$ energy values at $298 \mathrm{~K}$ are given in

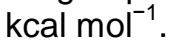<smiles>CC1=C[Si](C)(C)[Si](C)(C)[Ge](C=N)O1</smiles>

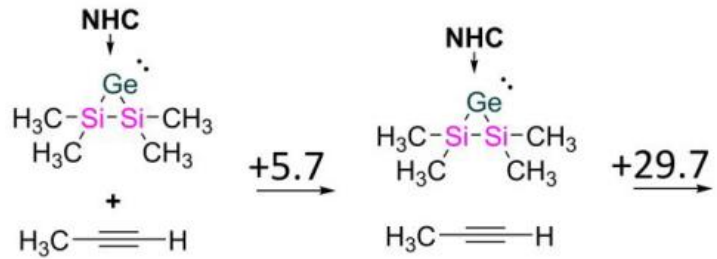

6b-Me

$+$

Ac

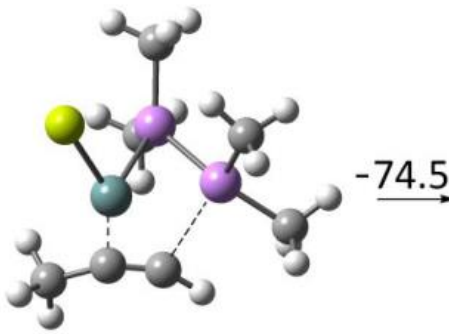

TS17
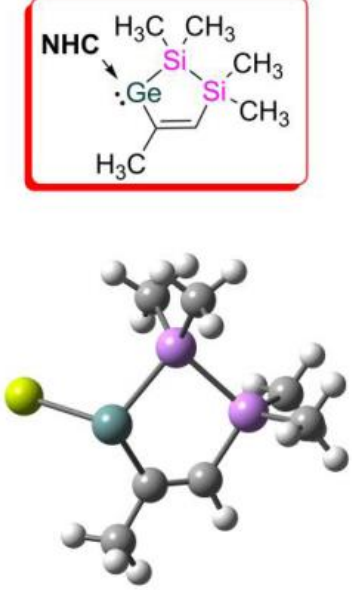

8-Me 
Figure S48. First proposed mechanism and intermediates for the formation of 9-Ph from $\mathbf{5 b - M e + P h e n y l a c e t y l e n e ~ u s i n g ~ s i m p l i f i e d ~ m o d e l ~ s y s t e m ~ w i t h ~ m e t h y l ~}$ groups instead of Tip, $\mathrm{Ph}$, and ${ }^{\prime} \mathrm{Pr}$ groups at B3LYP/6-31G(d,p) level of theory, respectively. $\Delta \mathrm{G}$ energy values at $298 \mathrm{~K}$ are given in $\mathrm{kcal} \mathrm{mol}^{-1}$.

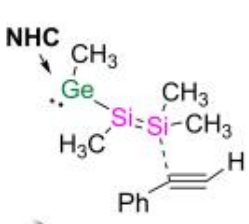

$\mathrm{NHC}, \mathrm{CH}_{3}$

$$
\begin{gathered}
: \mathrm{Ge}{ }^{\mathrm{C}} \mathrm{CH}_{3} \\
\mathrm{H}_{3} \mathrm{C}=\mathrm{CH}_{3} \mathrm{CH}_{3} \\
\mathrm{Ph} \stackrel{+}{=} \mathrm{H} \\
\begin{array}{c}
\mathbf{5 b}-\mathrm{Me} \\
\mathrm{Ac}-\mathrm{Ph}
\end{array}
\end{gathered}
$$

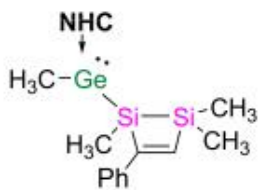

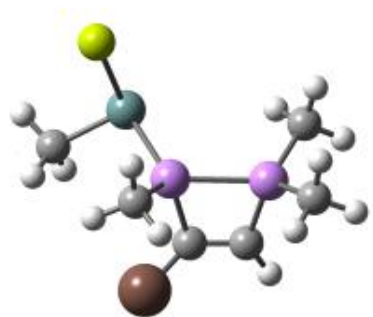

9-Ph

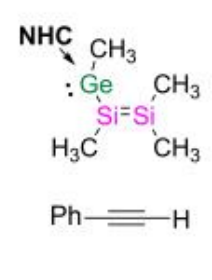

$15-\mathrm{Ph}$

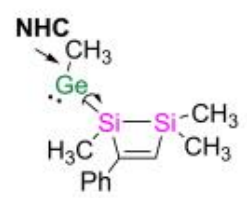

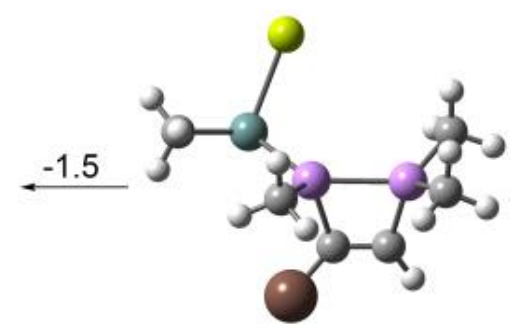

TS3-Ph
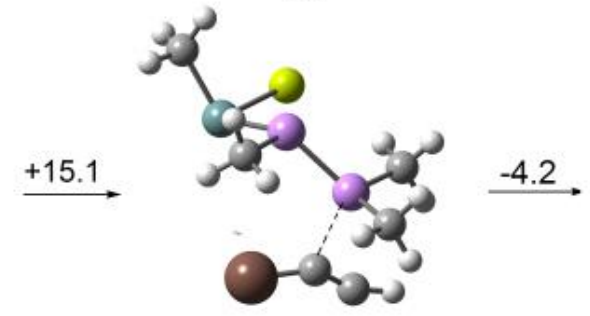

TS1-Ph
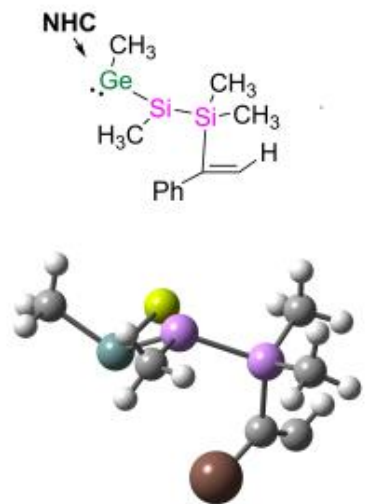

16-Ph
NHC

$$
\because \mathrm{Si}-\mathrm{Si}_{3}-\mathrm{CH}_{3}
$$$$
\mathrm{CH}_{3}
$$

$\stackrel{+4.2}{\longrightarrow}$

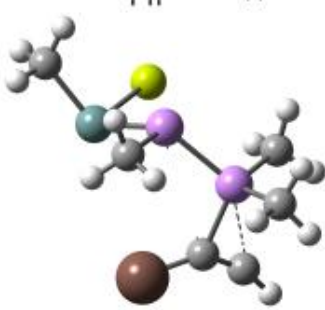

TS2-Ph

$-23.9$

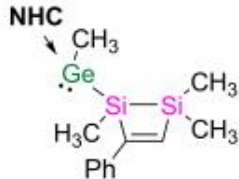

$\mathrm{Ph}$
NHC

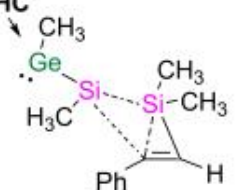

: Germanium

: Silicon

: Carbon

: Hydrogen

( NHC

: Pheny
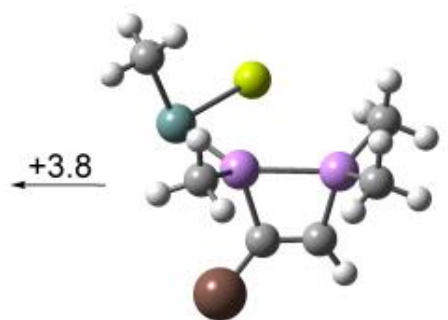

17-Ph



TS18-Ph
31-Ph 
Figure S49. First two steps of the alternative mechanism for the formation of $\mathbf{9 - P h}$ from $\mathbf{5 b}$-Me+ Phenylacetylene using simplified model system with methyl groups instead of Tip, $\mathrm{Ph}$, and 'Pr groups at B3LYP/6-31G(d,p) level of theory, respectively. $\Delta \mathrm{G}$ energy values at $298 \mathrm{~K}^{\prime}$ are given in kcal mol${ }^{-1}$.

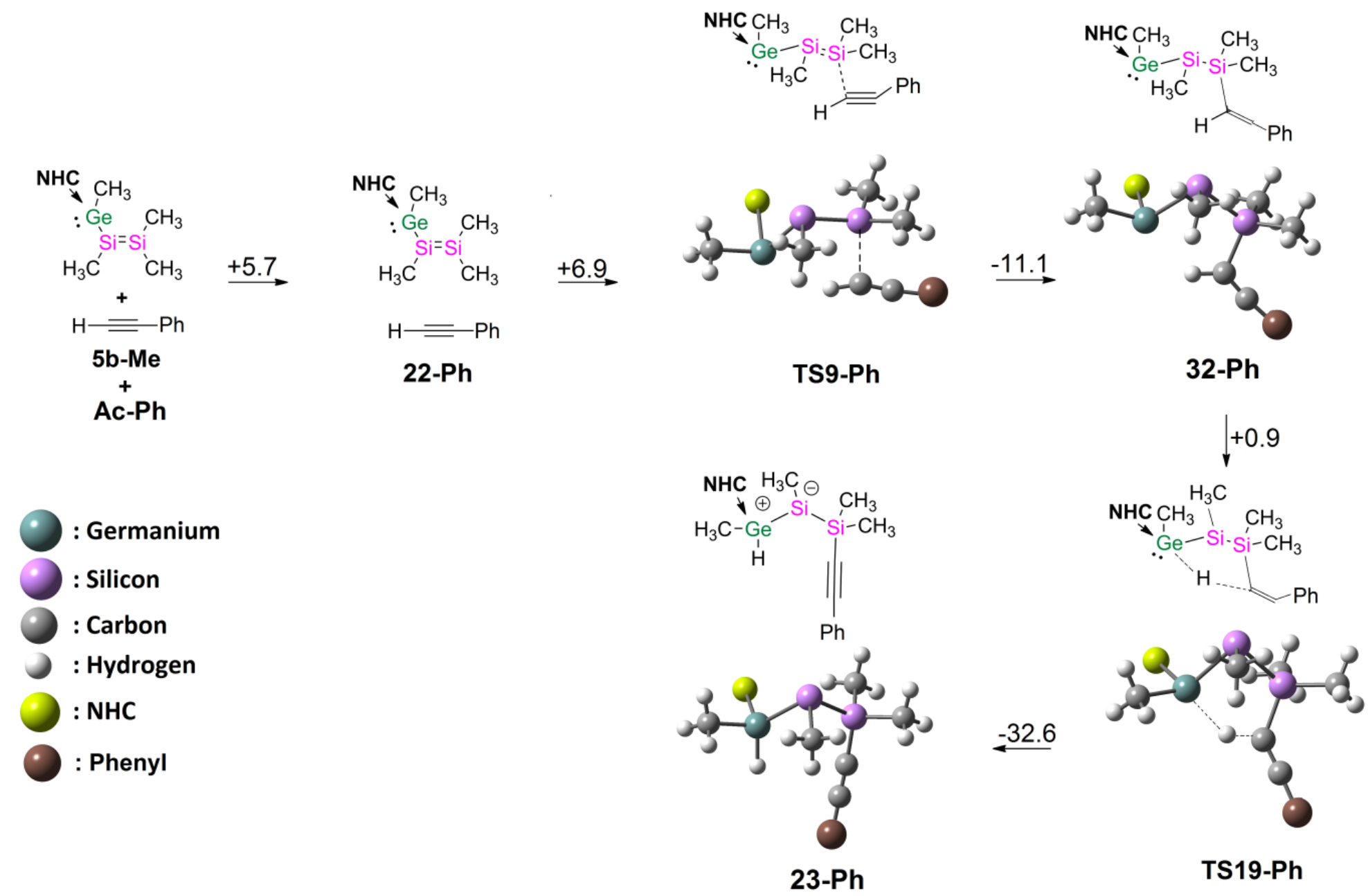


Figure S50. Proposed mechanism and intermediates for the formation of 7-Ph from NHC coordinated system using simplified model system with methyl groups instead of Tip, $\mathrm{Ph}$, and ${ }^{i} \mathrm{Pr}$ groups at B3LYP/6-31G(d,p) level of theory, respectively. $\Delta \mathrm{G}$ energy values at $298 \mathrm{~K}$ are given in kcal mol ${ }^{-1}$.

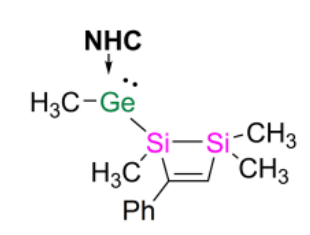

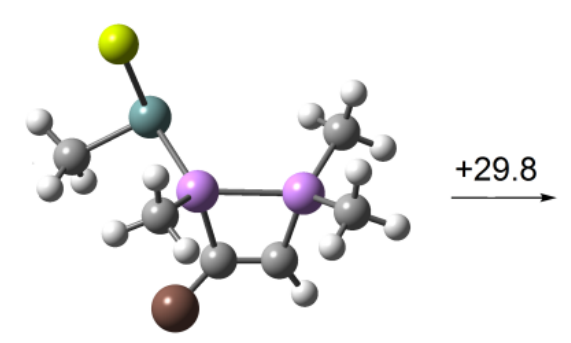

9-Ph
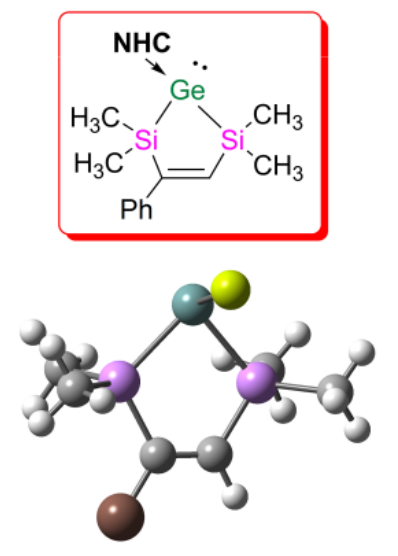

7-Ph

$-47.2$
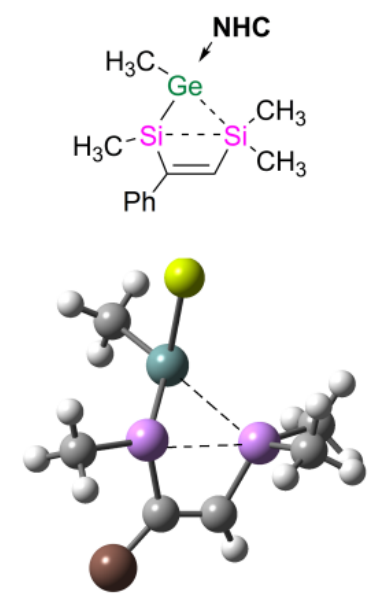

TS4-Ph
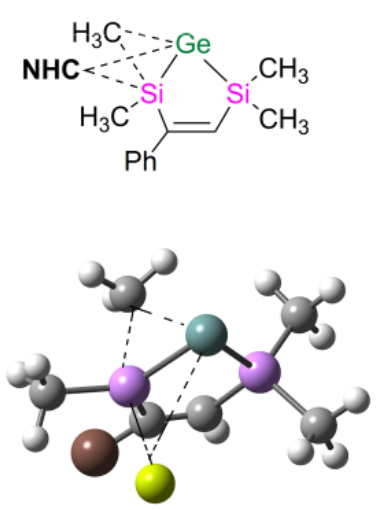

TS5-Ph
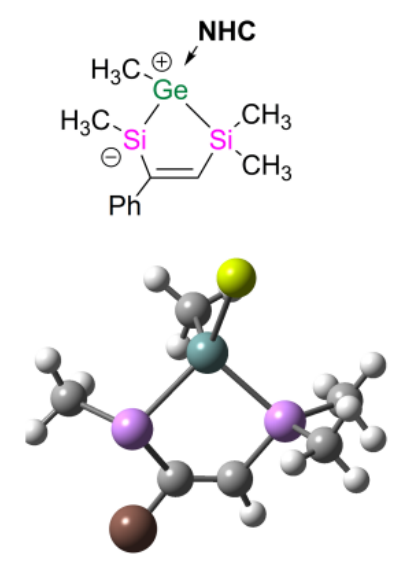

18-Ph

$\mid-3.3$

$$
\text { NHC }
$$

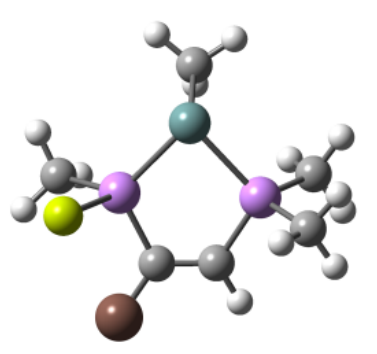

19-Ph

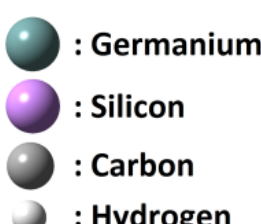

: Hydrogen

: NHC

: Phenyl 
Figure S51. Proposed mechanism and intermediates for the formation of 8-Ph from insertion of Phenylacetylene to Si-Ge single bond of $6 \mathrm{~b}-\mathrm{Me}$ using simplified model system with methyl groups instead of Tip, $\mathrm{Ph}$, and ${ }^{\prime} \mathrm{Pr}$ groups at B3LYP/6-31G(d,p) level of theory, respectively. $\Delta \mathrm{G}$ energy values at $298 \mathrm{~K}$ are given in $\mathrm{kcal} \mathrm{mol}{ }^{-1}$.
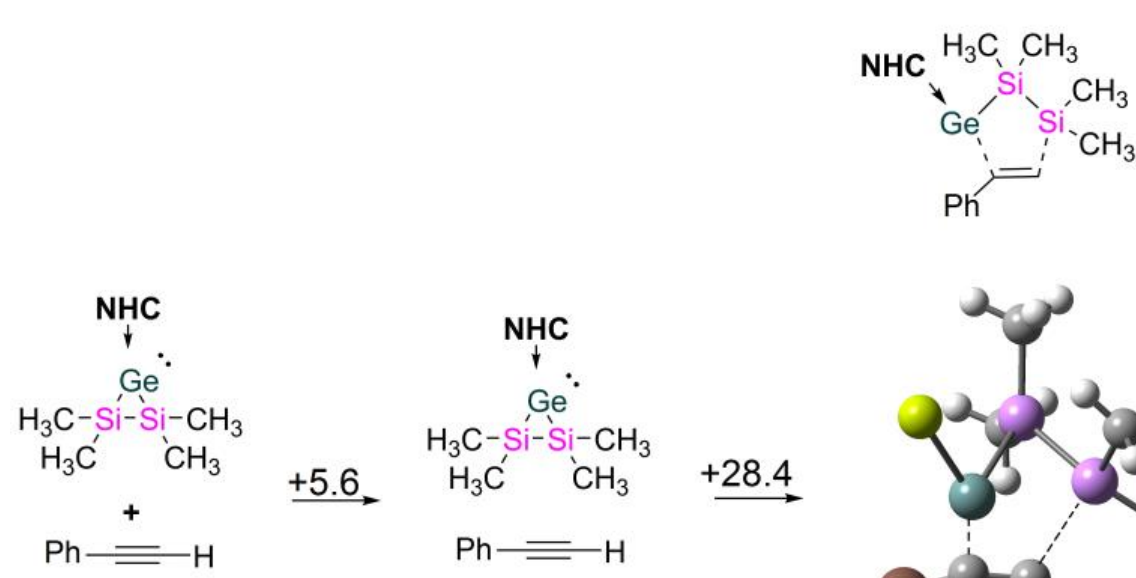

6b-Me

Ac-Ph $+\underline{28.4}$

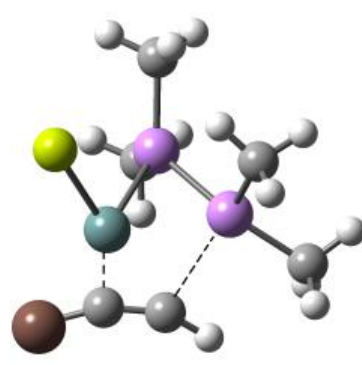

TS17-Ph
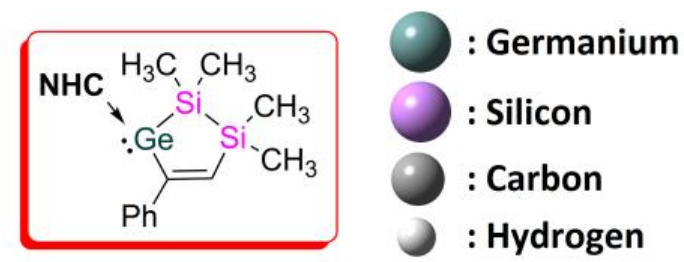

$-74.1$

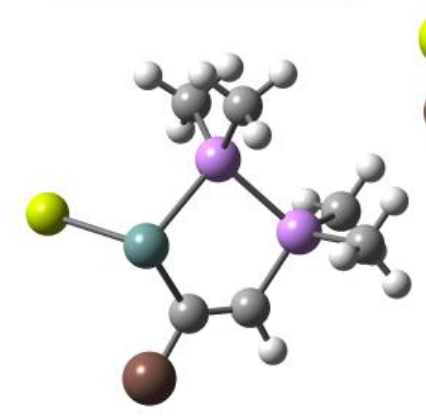

8-Ph 
Figure S52. Proposed mechanism and intermediates for the formation of 12-Ph from insertion of phenylisocyanide to Si-Si double bond of $5 \mathbf{b}$-Me using simplified model system with methyl groups instead of Tip, $\mathrm{Ph}$, and ${ }^{\prime} \mathrm{Pr}$ groups at B3LYP/6-31G(d,p) level of theory, respectively. $\Delta \mathrm{G}$ energy values at $298 \mathrm{~K}$ are given in $\mathrm{kcal} \mathrm{mol}^{-1}$
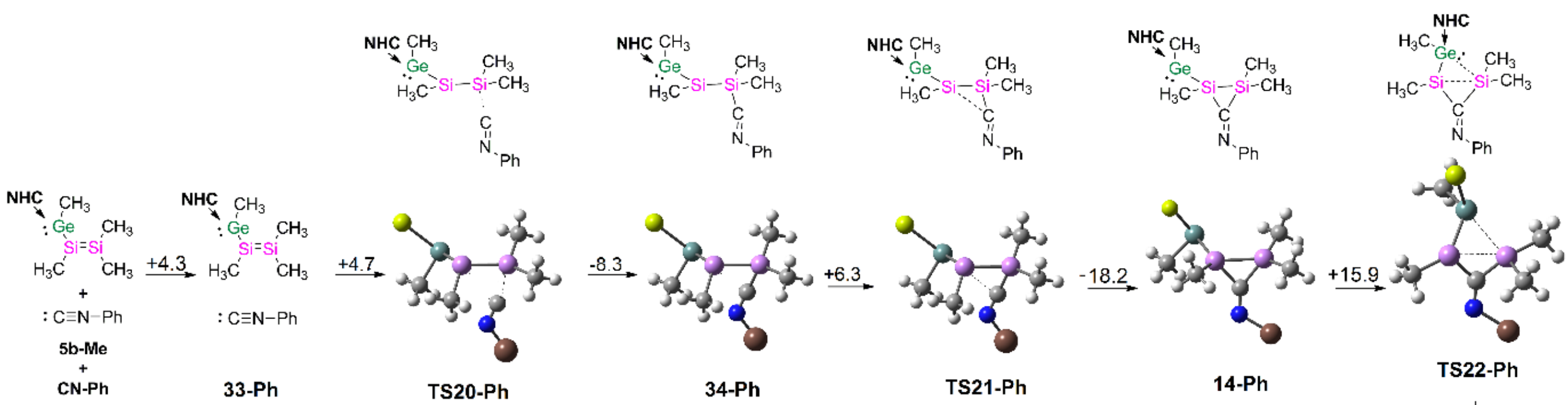

$\mathrm{CN}-\mathrm{Ph}$

33-Ph

34-Ph

TS21-Ph

14-Ph

TS22-Ph
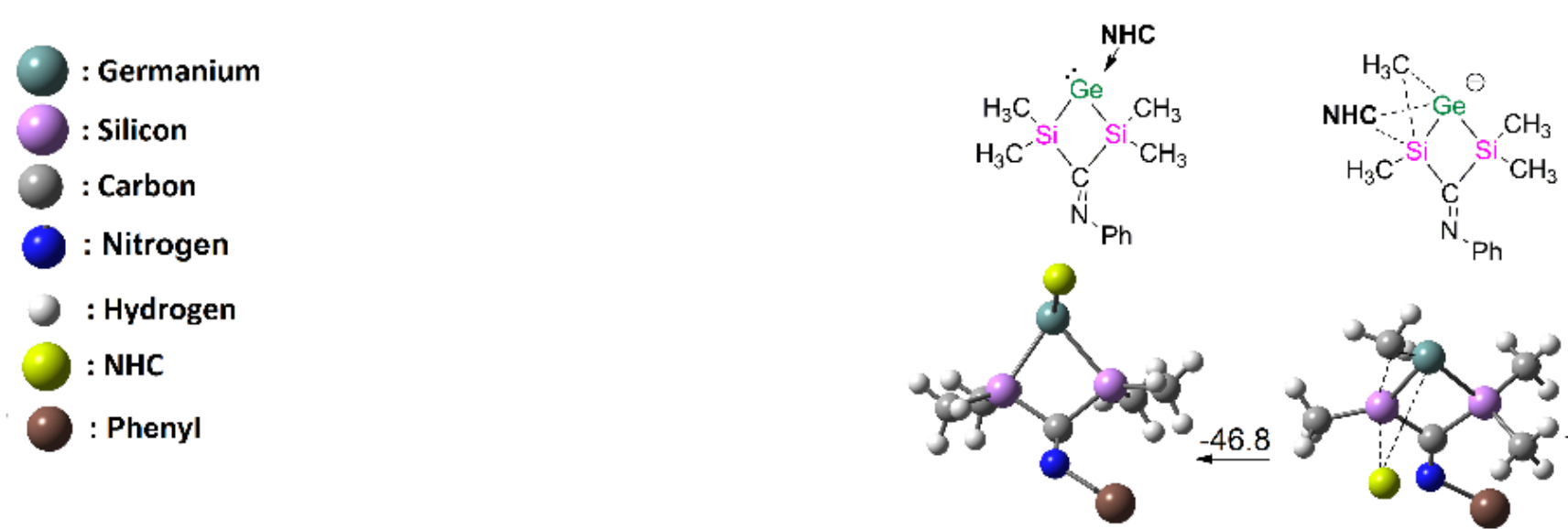

12-Ph

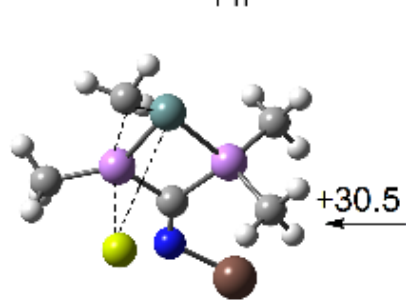

TS23-Ph

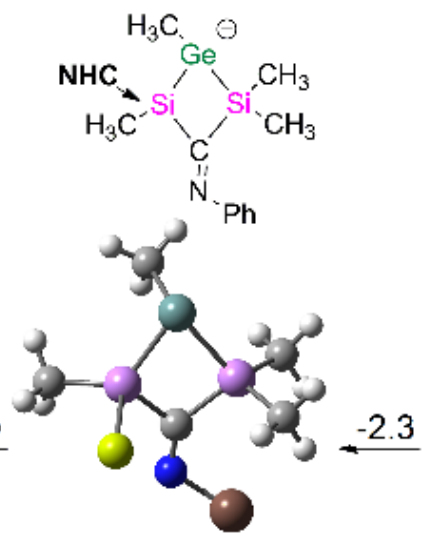

36-Ph

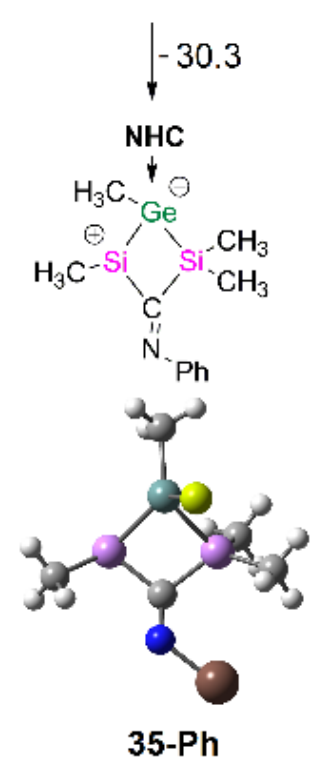


Figure S53. The IRC plot from TS1 to 16-Me at B3LYP/6-31G(d,p) level of theory.

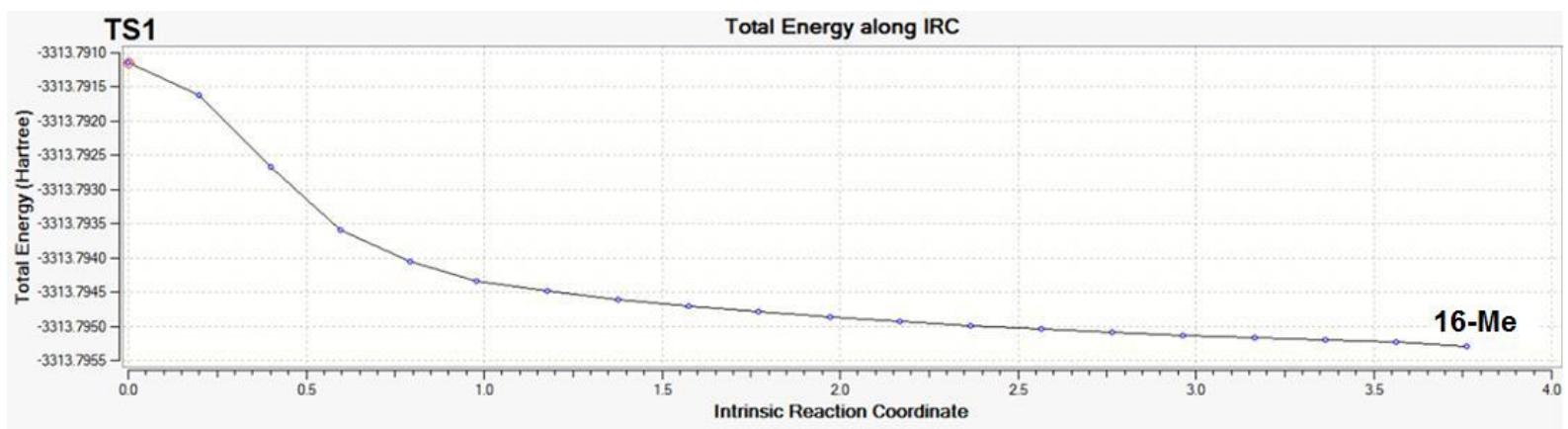

Figure S54. The IRC plot from 16-Me to 17-Me via TS2 at B3LYP/6-31G(d,p) level of theory.

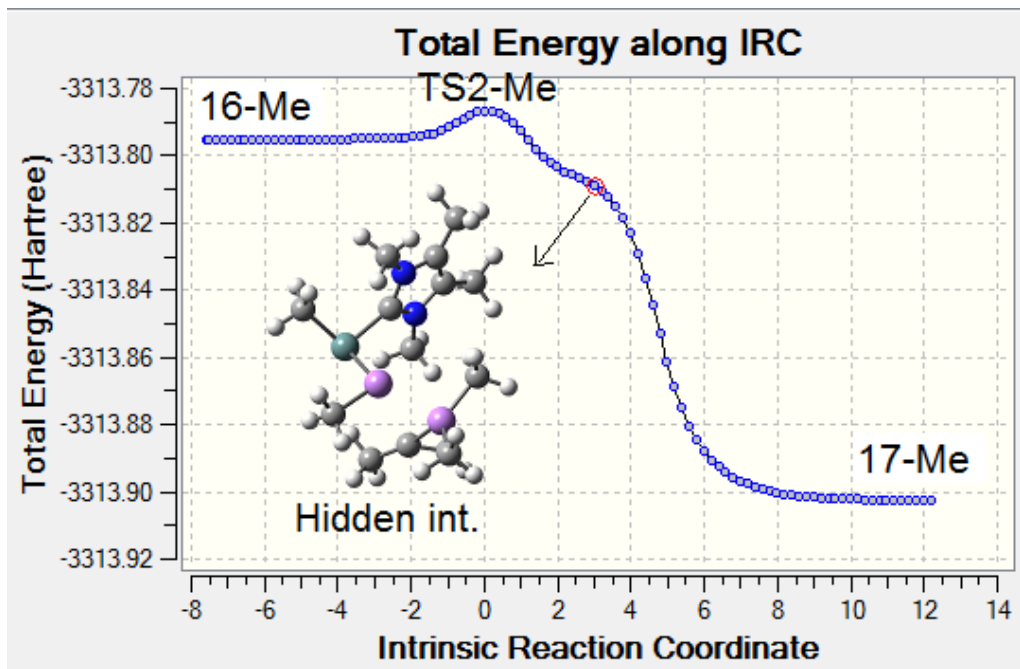

Figure S55. The IRC plot from 9-Me to 18-Me via TS4 at B3LYP/6-31G(d,p) level of theory.

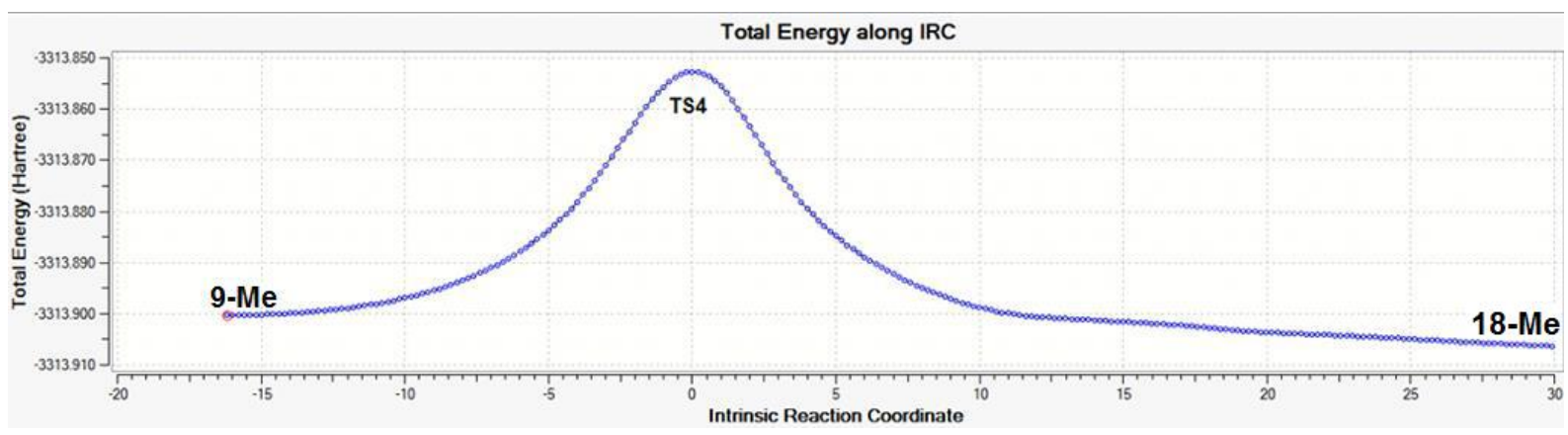

Figure S56. The IRC plot from TS5 to 19-Me at B3LYP/6-31G(d,p) level of theory.

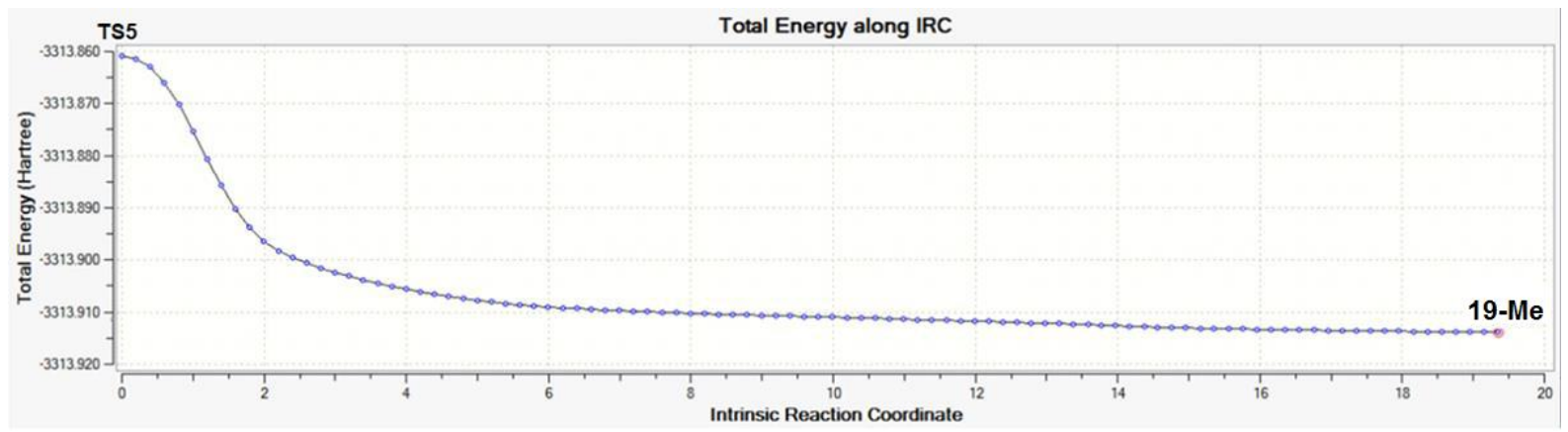


Figure S57. The IRC plot from TS5 to 7-Me at B3LYP/6-31G(d,p) level of theory.

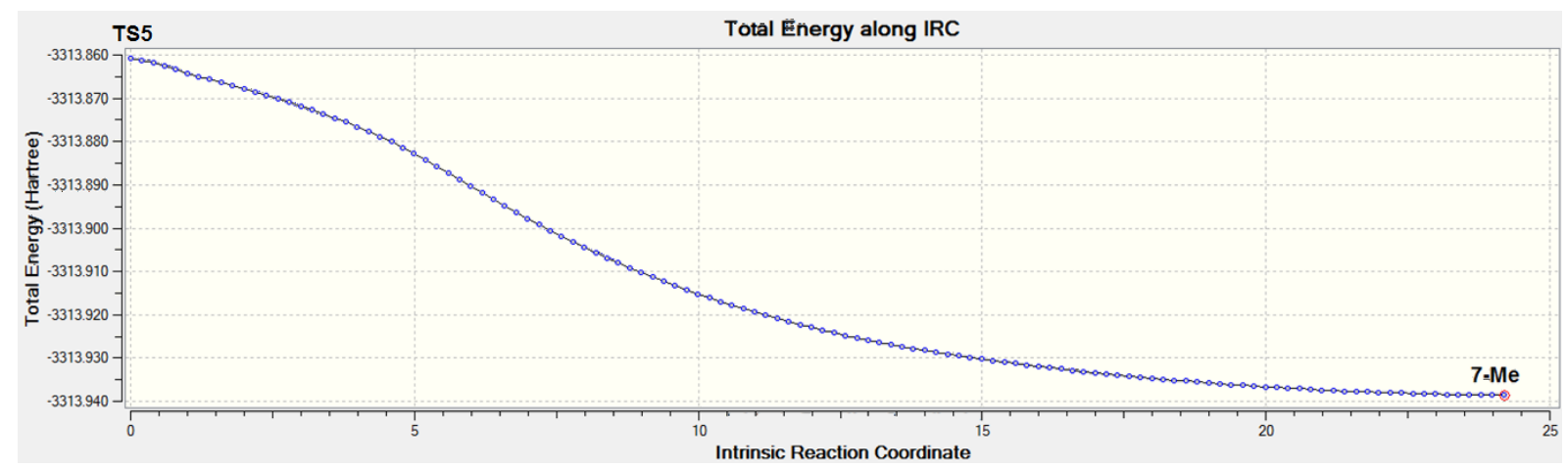

Figure S58. The IRC plot from 10-Me to 20-Me via TS6 at B3LYP/6-31G(d,p) level of theory.

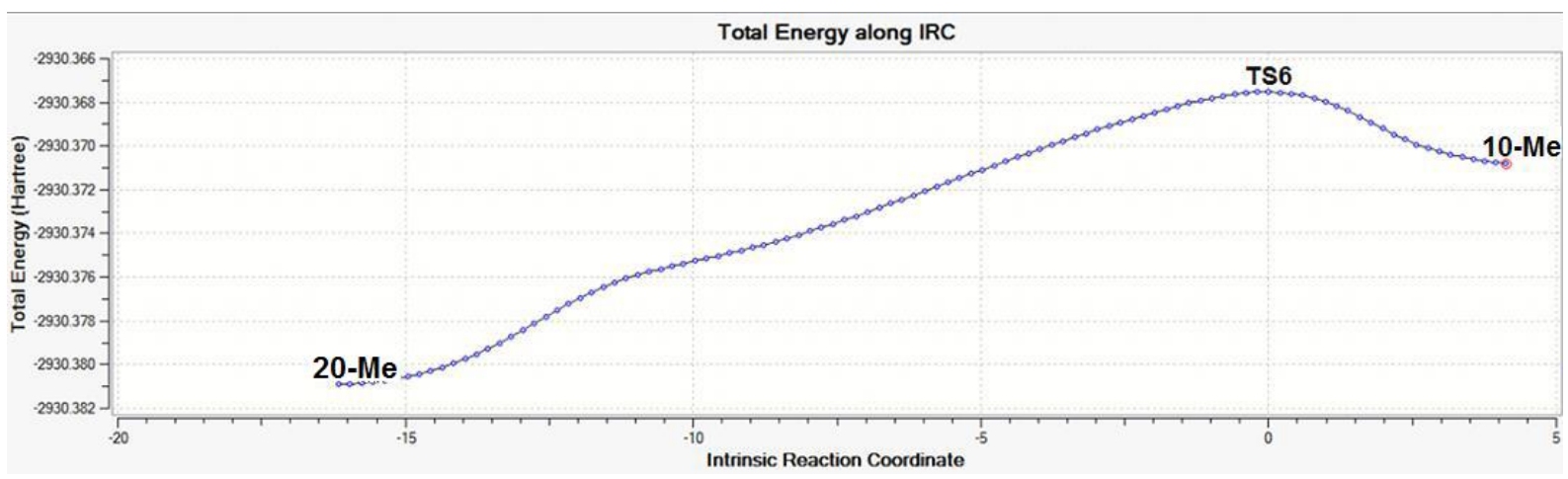

Figure S59. The IRC plot from 20-Me to 21-Me via TS7 at B3LYP/6-31G(d,p) level of theory.

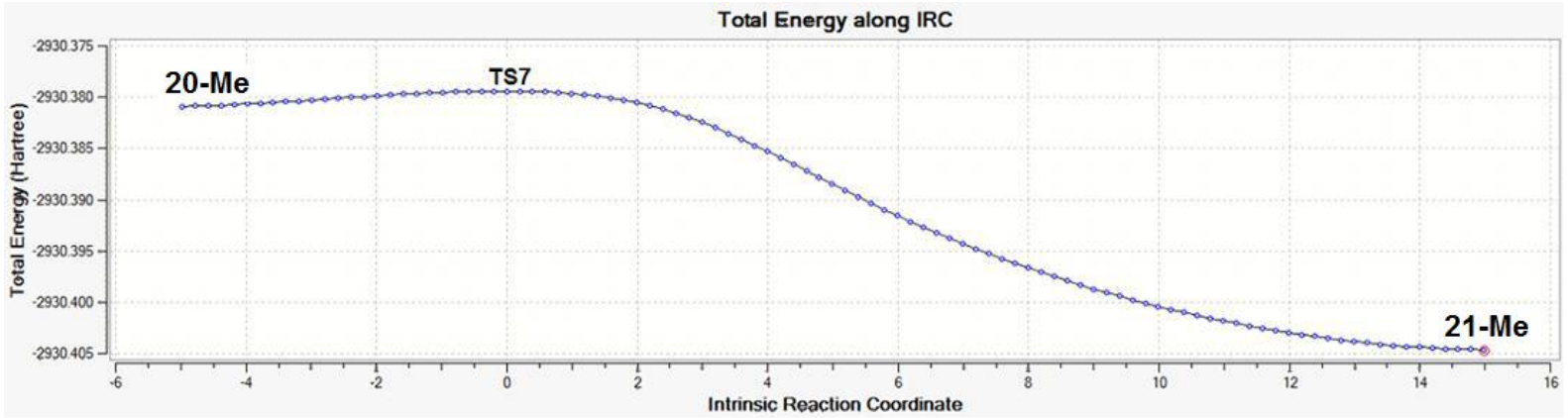

Figure S60. The IRC plot from 21-Me to 11-Me via TS8 at B3LYP/6-31G(d,p) level of theory.

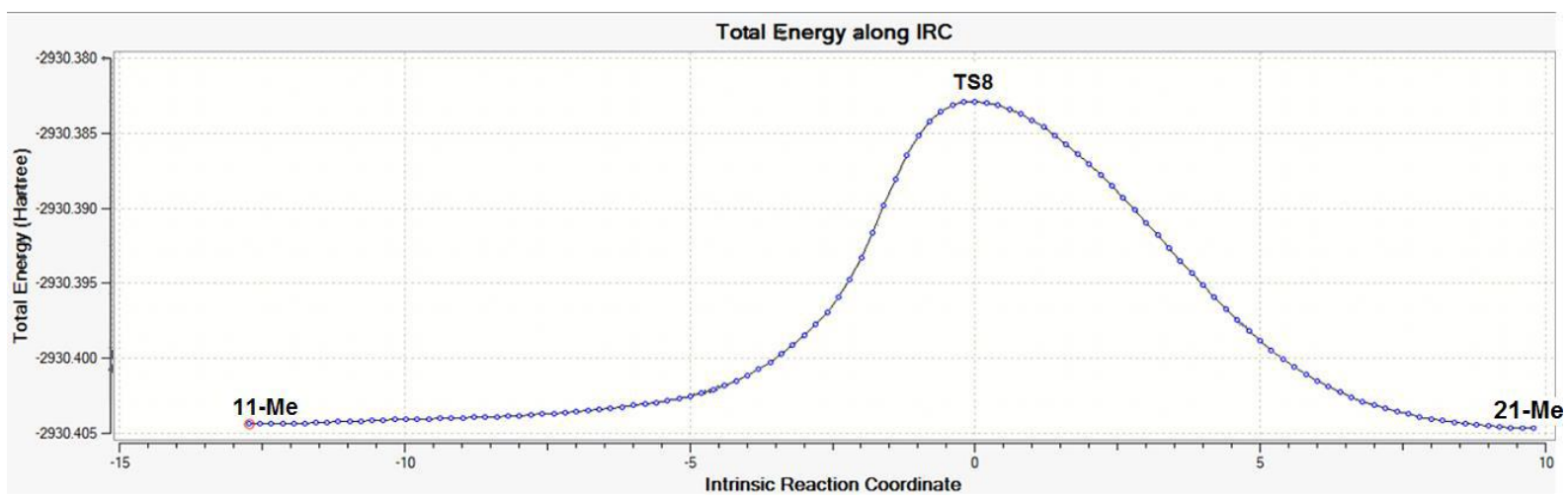


Figure S61. The IRC plot from TS9 to 23-Me at B3LYP/6-31G(d,p) level of theory.

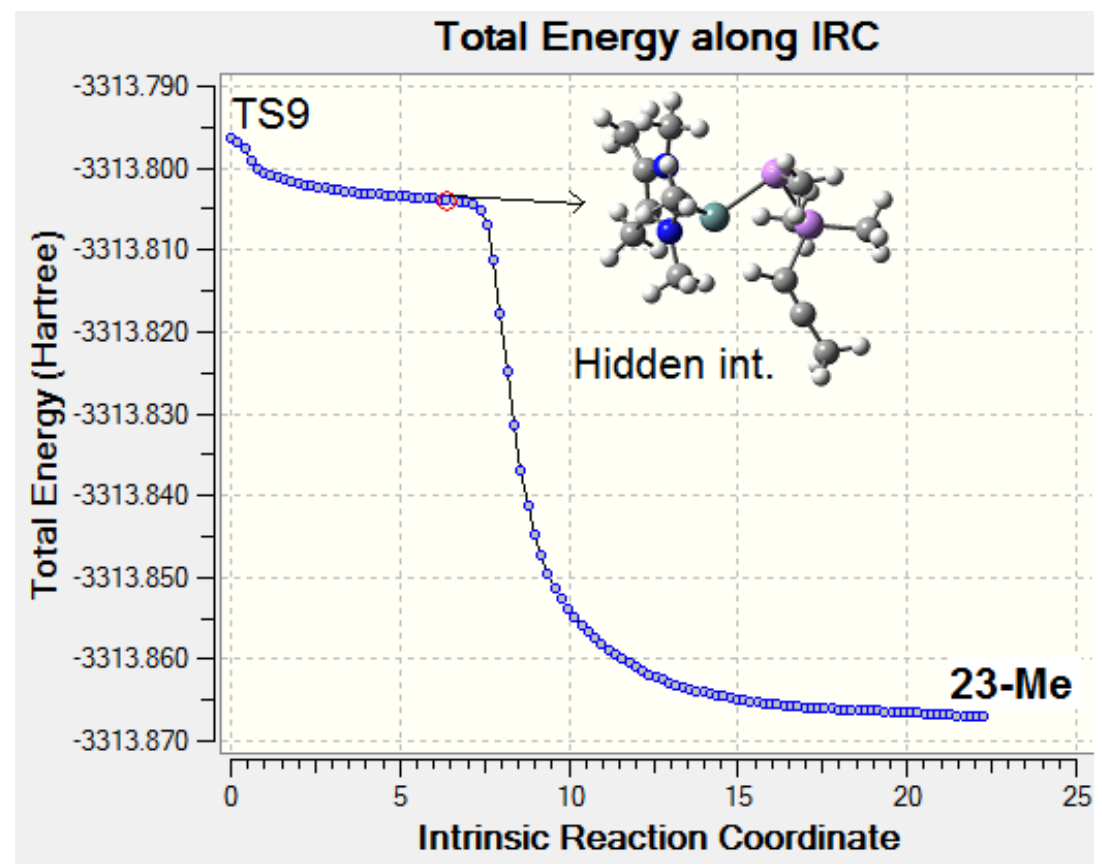

Figure S62. The IRC plot from 24-Me to 25-Me via TS11 at B3LYP/6-31G(d,p) level of theory.

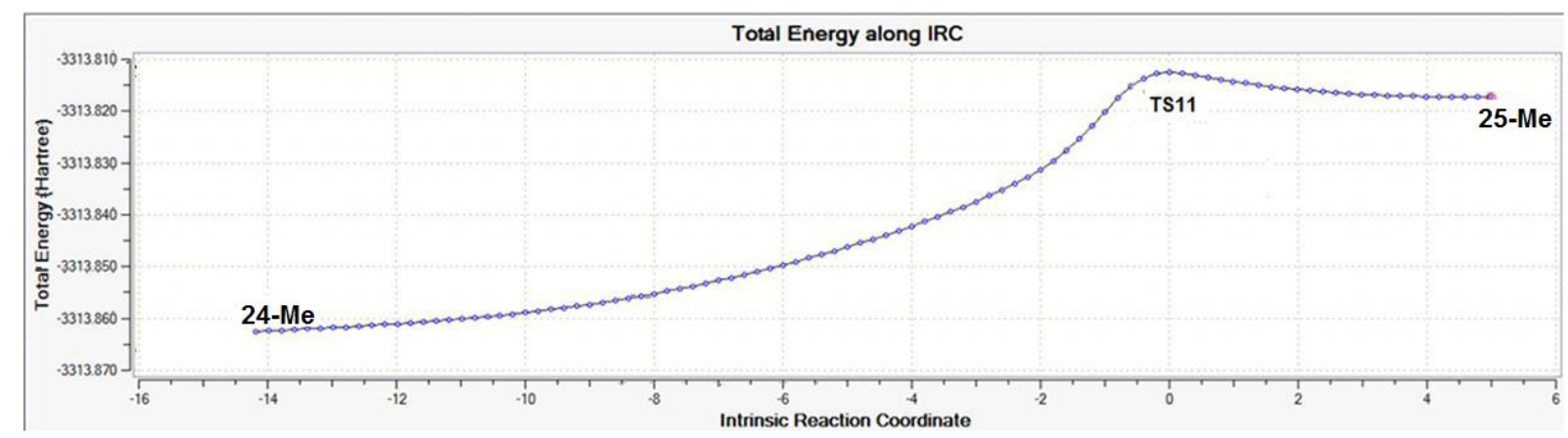

Figure S63. The IRC plot from TS12 to 25-Me at B3LYP/6-31G(d,p) level of theory.

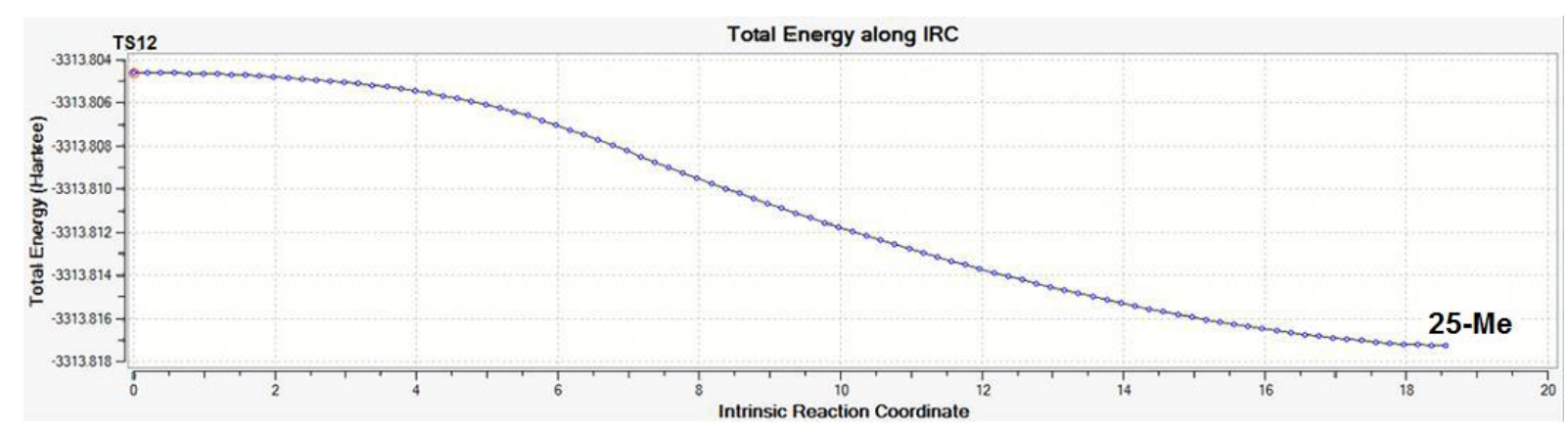


Figure S64. The IRC plot from TS13 to 26-Me at B3LYP/6-31G(d,p) level of theory.

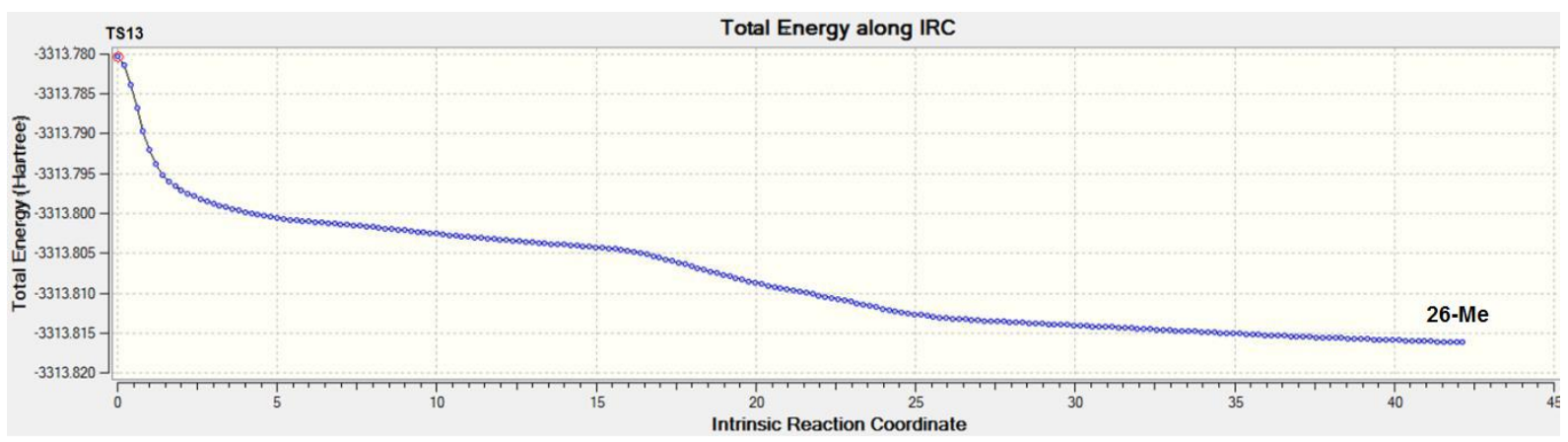

Figure S65. The IRC plot from TS13 to 9-Me at B3LYP/6-31G(d,p) level of theory.

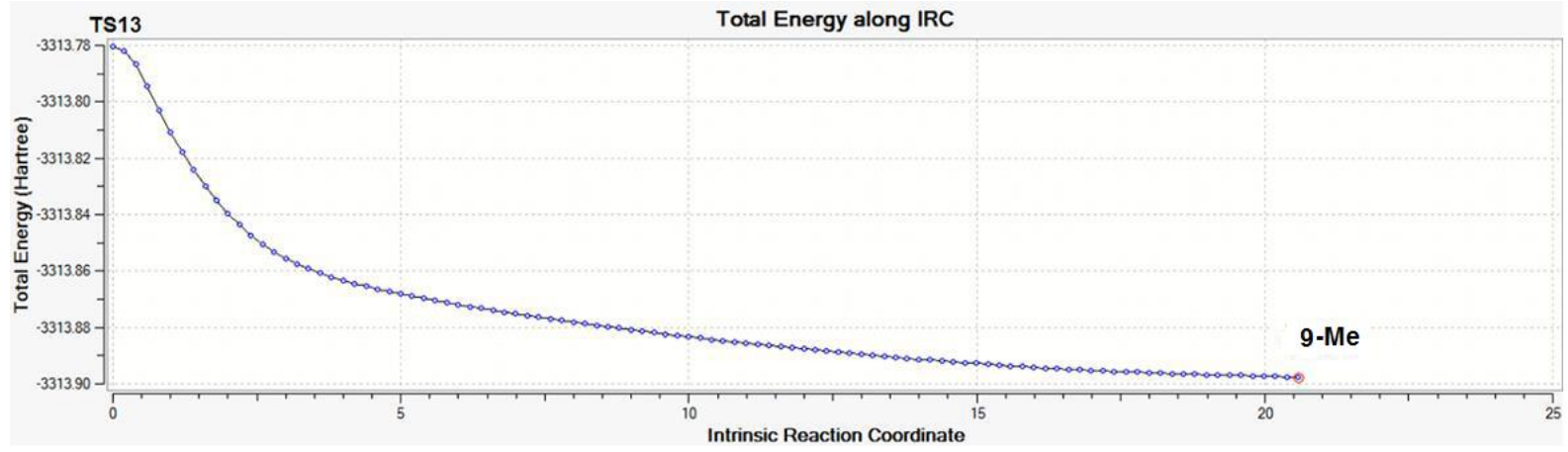

Figure S66. The IRC plot from TS14 to 28-Me at B3LYP/6-31G(d,p) level of theory.

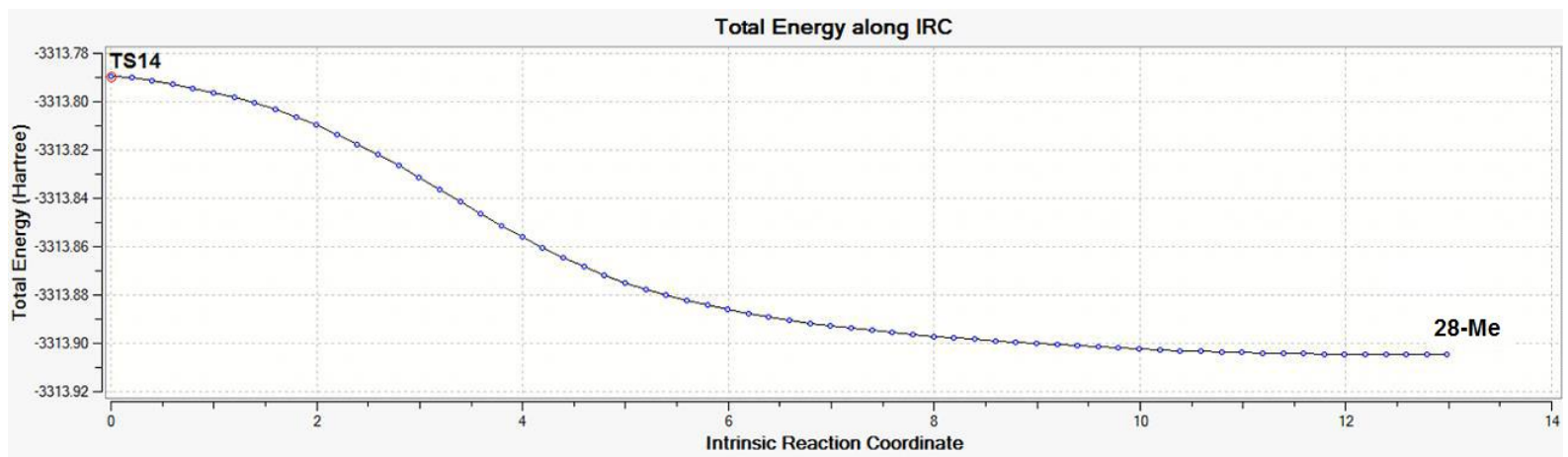

Figure S67. The IRC plot from 30-Me to 8-Me via TS17 at B3LYP/6-31G(d,p) level of theory.

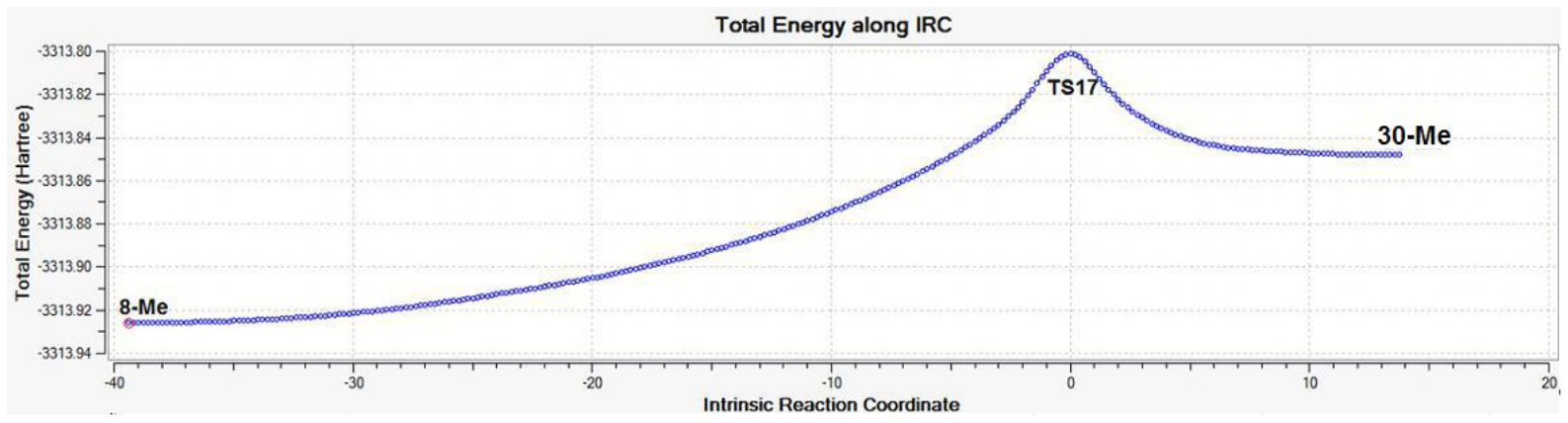


Table S1. Cartesian coordinates and energy values for the $\mathbf{5 b}$-Me.

Ge,2.2979396207,9.136855472,5.7244135971

$\mathrm{Si}, 4.1039427039,7.5629311306,5.5593312957$

Si,6.0644956695,8.4707143591,5.453504971

$\mathrm{N}, 0.3045905133,7.5426752669,7.5808942842$

$\mathrm{N}, 1.8446280451,8.6340258698,8.6196444794$

$\mathrm{C}, 1.4199777321,8.2937438362,7.3692008395$

$\mathrm{C}, 0.0257250997,7.4187191361,8.944333352$

C, $1.0090387818,8.1001133807,9.601155451$

C, $-1.1482144071,6.6570611914,9.4633676717$

$\mathrm{H},-1.1920746653,6.7254175192,10.5521411046$

$\mathrm{H},-1.0981969697,5.592616427,9.2040970823$

$\mathrm{H},-2.0939250452,7.0470278735,9.0697080174$

C, $1.236281065,8.2962750545,11.0632329361$

$\mathrm{H}, 0.4682178223,7.7724823396,11.6356627052$

$\mathrm{H}, 1.1952446464,9.3538817428,11.3487365208$

$\mathrm{H}, 2.2093247369,7.9050172285,11.3811948163$

C,3.0457603813,9.4237009244,8.8688627124

$\mathrm{H}, 3.942103612,8.8299490543,8.6668777371$

$\mathrm{H}, 3.048858999,10.2802603513,8.1878325369$

$\mathrm{H}, 3.0467181173,9.7653486087,9.9036133877$

C,-0.4648107422,6.909635877,6.5201067959

$\mathrm{H},-1.3223110035,7.5235843006,6.2271365221$

$\mathrm{H}, 0.1876671459,6.7774025395,5.6614040012$

$\mathrm{H},-0.8242394731,5.9352752245,6.8563679779$

C, $4.0457838256,5.643474272,5.490622651$

$\mathrm{H}, 5.052672323,5.2269549928,5.3867907508$

$\mathrm{H}, 3.5981716227,5.2277255391,6.3992705393$

$\mathrm{H}, 3.4503114658,5.3041332532,4.6365038118$

C, $1.1047019061,8.6313697069,4.1725466706$

$\mathrm{H}, 1.568165278,9.1611778222,3.3338752347$

$\mathrm{H}, 1.0149950345,7.5796743316,3.8871422838$

$\mathrm{H}, 0.1056240435,9.0616308521,4.3016513893$

C,6.2303801573,10.3615477657,5.4793350621

$\mathrm{H}, 5.2300155124,10.7957376511,5.5725168062$

$\mathrm{H}, 6.8436880626,10.7085219367,6.3182600388$

$\mathrm{H}, 6.6843713296,10.7369195768,4.5556738344$

C, $7.7328300727,7.5808429215,5.252558805$

$\mathrm{H}, 7.5966581168,6.4956525643,5.241696384$

$\mathrm{H}, 8.2221281877,7.8708580818,4.3162251698$

$\mathrm{H}, 8.4131550356,7.8288763741,6.0747260129$
Zero-point correction=
Thermal correction to Energy=
Thermal correction to Enthalpy=
0.335434 (Hartree/Particle)
0.360262
0.361206
Thermal correction to Gibbs Free Energy=
Sum of electronic and zero-point Energies=
Sum of electronic and thermal Energies=
Sum of electronic and thermal Enthalpies=
0.279728
$-3196.807162$
$-3196.782335$
$-3196.781390$
Sum of electronic and thermal Free Energies=
$-3196.862869$

Table S2. Cartesian coordinates and energy values for the $6 \mathrm{~b}-\mathbf{M e}$.

Ge,-0.7388,-0.00015,-1.263226

$\mathrm{Si},-2.104824,1.161642,0.40801$

Si,-2.105084,-1.161343,0.408226

$\mathrm{N}, 1.876778,-1.079683,-0.210593$

$\mathrm{N}, 1.877002,1.079608,-0.210911$

C, $1.07312,0.000014,-0.430581$ 
C,3.163685, $0.682226,0.162026$

C,3.163585,-0.682453,0.162125

C,4.25398,1.648497,0.487039

$\mathrm{H}, 3.970353,2.327958,1.299023$

$\mathrm{H}, 4.533261,2.263632,-0.376767$

$\mathrm{H}, 5.149157,1.111263,0.806524$

C, $4.253805,-1.648783,0.487208$

$\mathrm{H}, 5.149218,-1.111546,0.806031$

$\mathrm{H}, 4.532602,-2.264419,-0.376392$

$\mathrm{H}, 3.970403,-2.327754,1.299685$

C,-3.560776,-2.162198,-0.30844

$\mathrm{H},-4.394235,-2.222122,0.400311$

$\mathrm{H},-3.925242,-1.697662,-1.229583$

$\mathrm{H},-3.250082,-3.184382,-0.555156$

C,-1.475098,-2.022412,1.997129

$\mathrm{H},-0.649392,-1.469688,2.455833$

$\mathrm{H},-2.282338,-2.094152,2.735035$

$\mathrm{H},-1.129017,-3.04245,1.792695$

C,-1.474523,2.022641,1.996819

$\mathrm{H},-0.648476,1.470083,2.455111$

$\mathrm{H},-1.128792,3.042779,1.792292$

$\mathrm{H},-2.281466,2.094114,2.735073$

C, $-3.560728,2.162452,-0.308301$

$\mathrm{H},-4.394245,2.221849,0.400426$

$\mathrm{H},-3.250303,3.18483,-0.554553$

$\mathrm{H},-3.925014,1.69821,-1.229665$

C, $1.439824,2.450826,-0.433282$

$\mathrm{H}, 0.973998,2.524748,-1.420044$

$\mathrm{H}, 0.705347,2.747499,0.317647$

$\mathrm{H}, 2.301458,3.116494,-0.38727$

C, $1.43938,-2.450764,-0.433373$

$\mathrm{H}, 0.700443,-2.745255,0.313991$

$\mathrm{H}, 0.97869,-2.525858,-1.422484$

$\mathrm{H}, 2.299987,-3.117308,-0.381537$

Zero-point correction=

Thermal correction to Energy=

Thermal correction to Enthalpy=

Thermal correction to Gibbs Free Energy=

Sum of electronic and zero-point Energies=

Sum of electronic and thermal Energies=

Sum of electronic and thermal Enthalpies=

0.335977 (Hartree/Particle)

0.360319

0.361263

Sum of electronic and thermal Free Energies=

0.282525

$-3196.844927$

$-3196.820585$

$-3196.819641$

$-3196.898379$

Table S3. Cartesian coordinates and energy values for the7-Me.

Ge,-0.7109725507,-0.8239646846,-0.5008138478

Si,-1.3312856912,-0.6941556869,1.8399477945

Si,-1.4844726843,1.4660044073,-0.6545934979

$\mathrm{N}, 2.1972693591,0.2358350382,0.2455767598$

$\mathrm{N}, 2.142758732,-1.4161405895,-1.1280258127$

C,-1.8509006088,1.1096838418,2.1285409758

$\mathrm{H},-2.1647495251,1.4264325875,3.1328445417$

C,-1.9183267403,2.0231495981,1.1324991503

C, $1.3326007454,-0.5917173717,-0.4031358322$

C,3.5247846206, $-0.0625703375,-0.0657621924$

C,3.4885407558,-1.1109420913,-0.9376197501

C,4.678137284,0.6905632448,0.5083605916

C,4.5879278816,-1.8598517245,-1.6135928223

$\mathrm{H}, 4.7045248387,0.6266479458,1.6022641774$ 
$H, 4.6456897225,1.753580376,0.2428907223$

H,5.619393112,0.285369949,0.1317411646

$\mathrm{H}, 5.559011062,-1.4589584073,-1.3166438618$

$\mathrm{H}, 4.5190934074,-1.7881046067,-2.7051482451$

$\mathrm{H}, 4.5747628621,-2.9246257823,-1.3543510728$

C,-2.401748536,3.4383647634,1.3765487425

$\mathrm{H},-2.6528059416,3.6167034909,2.4287384243$

$\mathrm{H},-1.6455234758,4.176846142,1.0791378852$

$\mathrm{H},-3.2937077641,3.6603476191,0.7760675609$

C,-2.8777243815,-1.7889996377,2.057099727

$\mathrm{H},-3.5624766912,-1.6473613774,1.2143205109$

$\mathrm{H},-2.6002009113,-2.8489594211,2.0747416837$

$\mathrm{H},-3.4178091145,-1.5636927905,2.9841410778$

C,1.8241059099,1.3108887237,1.1550171077

$\mathrm{H}, 2.1885376043,2.2692906531,0.7748243603$

$\mathrm{H}, 0.7406276805,1.3491431698,1.2418229561$

$\mathrm{H}, 2.2555706037,1.1347067582,2.144180653$

C, $1.6742573108,-2.4876303082,-2.0020476286$

$H, 2.0145111425,-2.315156649,-3.0267701022$

$\mathrm{H}, 2.0464664258,-3.4537786762,-1.6503849579$

$\mathrm{H}, 0.5815302559,-2.4695964795,-1.9602084004$

C,-0.108167029,-1.219366595,3.210860307

$\mathrm{H},-0.5723140474,-1.0949640444,4.1970893722$

$\mathrm{H}, 0.1629242862,-2.2756838424,3.1065581447$

$\mathrm{H}, 0.817056567,-0.634792503,3.2050722969$

C,-3.1245237385,1.3983558142,-1.6288332627

$\mathrm{H},-3.7167171185,0.5325219681,-1.3147771538$

$\mathrm{H},-3.7303246346,2.3022073233,-1.4945628231$

$\mathrm{H},-2.9267766338,1.281465927,-2.7002889314$

C,- $-0.4249170912,2.8157136366,-1.4983198496$

$\mathrm{H}, 0.5128448479,3.018697068,-0.9713245293$

$\mathrm{H},-0.1743124751,2.520729437,-2.5230489374$

$H,-0.9825586334,3.758504124,-1.5555361775$

Zero-point correction $=$

Thermal correction to Energy=

Thermal correction to Enthalpy=

Thermal correction to Gibbs Free Energy=

Sum of electronic and zero-point Energies=

Sum of electronic and thermal Energies=

Sum of electronic and thermal Enthalpies=

Sum of electronic and thermal Free Energies=
0.395807 (Hartree/Particle)

0.423608

0.424552

0.338114

$-3313.542954$

$-3313.515152$

$-3313.514208$

$-3313.600647$

Table S4. Cartesian coordinates and energy values for the 8-Me.

Ge,-0.0229474946,0.8736627705,-1.2767769662

Si,-3.1324676791,-0.1178162976,-0.0691250084

Si,-1.3100012763,1.1404644698,0.7671058857

$\mathrm{N}, 2.7635910684,0.816794204,-0.2267859221$

$\mathrm{N}, 2.0343861854,-1.2059985832,-0.104567915$

C,-1.0314200184,-0.7483399975,-1.9080765777

C,-2.2802259254,-1.0395272426,-1.4722183196

C, $1.6561618172,0.0533287238,-0.4592462298$

C,3.8172424694,0.0482097824,0.2689622744

C,3.3593433338,-1.2345324199,0.3380177631

C,5.1472086614,0.6286404143,0.6172999907

C, $4.0329767099,-2.4906105573,0.7797057998$

$\mathrm{H}, 5.0604693588,1.421176655,1.3693656504$

$\mathrm{H}, 5.6505310197,1.0589930855,-0.2565211993$

H,5.8018560709,-0.1440459894,1.0252294912 
$\mathrm{H}, 5.0634546759,-2.284155635,1.0755222076$

$\mathrm{H}, 4.0614794032,-3.2405581273,-0.0192964083$

$\mathrm{H}, 3.5281316335,-2.9471283301,1.6390859104$

C,-3.923909729,-1.3548617593,1.1491939604

$\mathrm{H},-3.1712746603,-2.0050947389,1.6075669953$

$\mathrm{H},-4.4469682299,-0.8319553295,1.957736881$

$\mathrm{H},-4.654635254,-1.9966096227,0.6423711759$

C,2.8196202387, 2.2590965355,-0.4288261703

$\mathrm{H}, 2.0377019749,2.5247324243,-1.1472476596$

$\mathrm{H}, 2.6380626245,2.7929030122,0.5089835347$

$\mathrm{H}, 3.7988099137,2.5405296393,-0.8211362295$

C,1.1493569132,-2.3629615448,-0.0792288591

$\mathrm{H}, 0.1409644355,-2.0229712375,-0.3096228375$

$\mathrm{H}, 1.4588696329,-3.107136865,-0.8189815329$

$\mathrm{H}, 1.1643433621,-2.8206015408,0.9137852873$

C,-4.5326589367,0.9718709193,-0.7671663151

$\mathrm{H},-5.2765049816,0.3643602618,-1.2959273417$

$\mathrm{H},-5.051768681,1.5178276819,0.0284120628$

$\mathrm{H},-4.13800271,1.7059662047,-1.4769637607$

C,-1.7319196642,2.9716435723,1.1167278715

$\mathrm{H},-2.1074770401,3.4624514615,0.2133662771$

$\mathrm{H},-2.4891811545,3.0708450133,1.9026305066$

$\mathrm{H},-0.8423756506,3.5229403396,1.4433038235$

C,- $-0.6168344114,0.4105062311,2.3988779858$

$\mathrm{H},-0.4402257852,-0.6669530065,2.3184456459$

$\mathrm{H}, 0.3312584432,0.8860733838,2.6750474874$

$\mathrm{H},-1.3186589832,0.5703765026,3.2258667931$

C, $-0.431481328,-1.4590244284,-3.1026624668$

$\mathrm{H}, 0.6245468701,-1.7070821866,-2.9331855309$

$\mathrm{H},-0.9715794003,-2.3760151799,-3.3706952909$

$\mathrm{H},-0.4372613139,-0.7914621267,-3.9739698477$

$\mathrm{H},-2.8349545084,-1.8227955421,-2.0077698725$

Zero-point correction=

Thermal correction to Energy=

Thermal correction to Enthalpy=

Thermal correction to Gibbs Free Energy=

Sum of electronic and zero-point Energies=

Sum of electronic and thermal Energies=

Sum of electronic and thermal Enthalpies=

Sum of electronic and thermal Free Energies=
0.395602 (Hartree/Particle)

0.423404

0.424348

0.338463

$-3313.530674$

$-3313.502872$

$-3313.501928$

$-3313.587814$

Table S5. Cartesian coordinates and energy values for the 9-Me.

Ge,0.1004041802,1.092524222,1.3693884021

$\mathrm{Si},-2.744146318,0.4385732475,-1.4658182138$

Si,-1.4366300991,-0.4439087409,0.2942843773

$\mathrm{N}, 2.2395570244,1.0117439739,-0.7179582162$

$\mathrm{N}, 2.7826155939,-0.4391066784,0.7773614047$

C,-3.1941433052,-0.1331814612,1.0239412784

C,-3.9277527716,0.3731366288,-0.0013686334

C, $1.7917235378,0.4301727717,0.4326624167$

C,3.4908725309,0.5169185604,-1.085984804

C,3.8424683672,-0.3934547339,-0.1329111762

C,4.2136753766,0.9714960142,-2.3099028427

C,5.0704111153,-1.2302733115,0.0075125721

$\mathrm{H}, 4.460435227,2.0389984836,-2.2705086666$

$\mathrm{H}, 3.6221992792,0.8039568493,-3.2171880746$

$\mathrm{H}, 5.1508722944,0.4226027622,-2.4210292103$

$\mathrm{H}, 5.7772426366,-0.9997106252,-0.7920442056$ 
$\mathrm{H}, 4.8493031423,-2.3028284075,-0.0504459098$ $\mathrm{H}, 5.5800827802,-1.050395494,0.9609588471$ C,-2.3644536526,2.2103312702,-2.0473702053 $\mathrm{H},-1.9555944817,2.7899328706,-1.2133669501$ $\mathrm{H},-1.6314422822,2.2200026597,-2.8628147857$ $\mathrm{H},-3.2652688791,2.7189729368,-2.409892224$ C, $1.4805539789,2.0004636213,-1.4715188471$ $\mathrm{H}, 2.1638815625,2.6911560357,-1.9679983974$ $\mathrm{H}, 0.8428793622,1.5197027665,-2.2184228156$ $\mathrm{H}, 0.8467046183,2.5444146773,-0.763290047$ C,2.7082192632,-1.3493303527,1.9112350241 $\mathrm{H}, 3.2559322913,-2.2645466546,1.6814969628$ H,3.128598781,-0.899308132,2.8152056242 $\mathrm{H}, 1.6627642086,-1.5895718893,2.0884479746$ C,-3.2502013761,-0.59857023,-2.9785749553 $\mathrm{H},-4.1336157943,-0.1724976482,-3.4689954508$ $\mathrm{H},-2.4455416675,-0.634293004,-3.7219742884$ $\mathrm{H},-3.4895849535,-1.6277808954,-2.6938286245$ C, $0.2992336089,0.3622365284,3.2506584038$ $\mathrm{H}, 1.2532423553,0.6986953043,3.670688813$ $\mathrm{H},-0.480020965,0.877345037,3.8219100853$ $\mathrm{H}, 0.1866113627,-0.7111871734,3.4279014699$ C,-1.051789894,-2.3241804822,0.2343834486 $\mathrm{H},-0.8914698534,-2.7280096686,1.2408039876$ $\mathrm{H},-1.8818674376,-2.8778237483,-0.2183099176$ $\mathrm{H},-0.1500512671,-2.5248921108,-0.354646983$ C,-3.6743157736,-0.2940255275,2.4409062905 $\mathrm{H},-3.5282494127,-1.3195888087,2.8033147174$ $\mathrm{H},-3.0919182495,0.3557688933,3.1070131503$ $\mathrm{H},-4.7347640501,-0.0379597928,2.5540491972$ $\mathrm{H},-4.9664549954,0.6965994566,0.1274629971$
Zero-point correction $=$
Thermal correction to Energy= Thermal correction to Enthalpy=
0.395455 (Hartree/Particle) 0.423525 0.424469 Thermal correction to Gibbs Free Energy= Sum of electronic and zero-point Energies= Sum of electronic and thermal Energies= Sum of electronic and thermal Enthalpies= Sum of electronic and thermal Free Energies=
0.336077 $-3313.504918$ $-3313.476849$ $-3313.475905$ $-3313.564296$

Table S6. Cartesian coordinates and energy values for the 10-Me.

$\mathrm{Ge}, 1.7446914869,-0.4906942975,-0.843057101$ $\mathrm{Si},-0.003272658,0.2522398929,0.6697596325$ $\mathrm{Si},-2.0866274047,-0.4770429875,-0.1559446458$ C,-3.628149997,-0.2147117054,0.9202851237 $\mathrm{H},-3.7216570918,-1.0118039921,1.6659031466$ $\mathrm{H},-3.5894508566,0.7416295201,1.4501355376$ $\mathrm{H},-4.5375214224,-0.224209563,0.3082954481$ C,-2.2185758616,-2.1391004284,-1.0569981457 $\mathrm{H},-3.0771193361,-2.1570954547,-1.7382459403$ $\mathrm{H},-1.3144112706,-2.33108598,-1.6427792255$ $\mathrm{H},-2.3420861413,-2.9639060688,-0.3464496198$ C, $0.3003621776,0.9120331422,2.4338344082$ $\mathrm{H},-0.5642826412,1.47361489,2.8022977252$ $\mathrm{H}, 0.4835469201,0.0830111141,3.1253994025$ $\mathrm{H}, 1.1744357195,1.5710423744,2.4665917038$ C,3.3095243166,0.5253626653,-0.1117098209 $\mathrm{H}, 3.7918648135,-0.2157743426,0.5463849442$ 
$\mathrm{H}, 4.0279093742,0.7667518777,-0.8997362123$

$\mathrm{H}, 3.1026611905,1.4134508219,0.4902261382$

C,- $0.5334149705,1.5500593122,-0.6767205777$

C,-1.654317344,1.0140561964,-1.2335053072

$\mathrm{H},-2.1366419862,1.4572708379,-2.1106428246$

C, $0.1690981704,2.7942570695,-1.1602654668$

$\mathrm{H},-0.4181084925,3.3255946017,-1.9182242518$

$\mathrm{H}, 0.3696287656,3.4875608935,-0.3345660614$

$\mathrm{H}, 1.1444775398,2.5492426102,-1.6017650099$

Zero-point correction=

Thermal correction to Energy=

Thermal correction to Enthalpy=

0.208677 (Hartree/Particle)

0.225875

0.226819

Thermal correction to Gibbs Free Energy=

Sum of electronic and zero-point Energies=

Sum of electronic and thermal Energies=

Sum of electronic and thermal Enthalpies=

0.163127

$-2930.162193$

$-2930.144995$

$-2930.144051$

Sum of electronic and thermal Free Energies $=$

$-2930.207743$

Table S7. Cartesian coordinates and energy values for the 11-Me.

$\mathrm{Ge}, 0.7956225553,-1.5979470867,-0.1362652398$

$\mathrm{Si},-1.3453211189,-0.4892640003,0.281175488$

$\mathrm{Si}, 1.7219696948,0.6530386102,0.1434298406$

C,2.4028474956,1.0186314753,-1.6059214994

$\mathrm{H}, 3.3438667591,0.4855758676,-1.7769651715$

$\mathrm{H}, 1.6984636108,0.7058154622,-2.3853402053$

$\mathrm{H}, 2.586731041,2.0910733164,-1.7389645429$

C,3.1106548893,0.9112189878,1.4136701713

$\mathrm{H}, 3.4582061984,1.9510717668,1.3955539903$

$\mathrm{H}, 2.7715693489,0.6927550827,2.4311063883$

$\mathrm{H}, 3.9684958368,0.2663466566,1.1979593764$

C,-2.7775840748,-0.7606331971,-0.939022435

$\mathrm{H},-2.5556879247,-0.3375020894,-1.9239267866$

$\mathrm{H},-2.9795400167,-1.8283489378,-1.0707170332$

$\mathrm{H},-3.6965184277,-0.2883964693,-0.5724108517$

C,-1.8249127012,-1.2435475206,1.9740632044

$\mathrm{H},-2.2253108033,-2.2545619483,1.8466857363$

$\mathrm{H},-0.9605661991,-1.3140229661,2.6445363262$

$\mathrm{H},-2.5850337934,-0.6360971531,2.4785941579$

C, $-1.01154393,1.3763332495,0.5214469884$

C, $0.2698921627,1.805781838,0.5014517332$

$\mathrm{H}, 0.4576675165,2.8714124051,0.6842918622$

C,-2.172759128,2.3189930545,0.7585718441

$\mathrm{H},-1.8396348923,3.3486477225,0.9294310416$

$\mathrm{H},-2.8588895153,2.3241346915,-0.0980094814$

$\mathrm{H},-2.7691055836,2.008742182,1.6266620974$

Zero-point correction=

Thermal correction to Energy=

Thermal correction to Enthalpy=

Thermal correction to Gibbs Free Energy=

Sum of electronic and zero-point Energies=

Sum of electronic and thermal Energies=

Sum of electronic and thermal Enthalpies=

Sum of electronic and thermal Free Energies=
0.209900 (Hartree/Particle)

0.226825

0.227769

0.163995

$-2930.194534$

$-2930.177608$

$-2930.176664$

$-2930.240438$ 
Table S8. Cartesian coordinates and energy values for the 15-Me.

Ge,-0.1326264072,1.4901950895,-0.8338752524

$\mathrm{Si},-1.9956728804,-0.8058862288,2.0032727404$

$\mathrm{Si},-1.6328837838,1.1231881976,1.0155782771$

$\mathrm{N}, 2.488399452,0.5123969645,0.5822548965$

$\mathrm{N}, 1.4726549684,-0.9891815996,-0.5826482309$

C,-2.4574242442,-1.8030726511,-3.5483002634

C,-2.0267705125,-2.9046605832,-3.7987547404

C, $1.3740303232,0.2993766433,-0.1648473021$

C,3.2897845026, $-0.630279403,0.6260406501$

$\mathrm{C}, 2.6400456109,-1.5839632014,-0.107027108$

C, $4.5854007187,-0.6880425613,1.3651700889$

C, $3.0153310197,-2.9990854735,-0.3988292297$

$\mathrm{H}, 4.4522429188,-0.5522124318,2.445058785$

$\mathrm{H}, 5.2870397723,0.0794137501,1.0191841977$

$\mathrm{H}, 5.0609014101,-1.6593802267,1.2159839606$

$H, 3.9709388255,-3.2387763556,0.071588761$

$\mathrm{H}, 3.1213948524,-3.1825414693,-1.473954163$

$\mathrm{H}, 2.2720711604,-3.705305331,-0.012183551$

C, $-0.543152068,-1.8650567535,2.6439683096$

$\mathrm{H}, 0.3887974624,-1.5975390318,2.137541972$

$\mathrm{H},-0.3945560888,-1.7318339859,3.7214474658$

$\mathrm{H},-0.7281482652,-2.9308968431,2.4666995866$

C,2.7411895787, 1.7487375779,1.3119257426

$\mathrm{H}, 3.2217768147,2.4956382777,0.6743551218$

$\mathrm{H}, 1.7875421045,2.139470154,1.6648656761$

$\mathrm{H}, 3.3855409413,1.5407564736,2.1664699014$

C, $0.4530079225,-1.6331167336,-1.4100620465$

$\mathrm{H},-0.5161393903,-1.578928166,-0.9077073877$

$\mathrm{H}, 0.3733748181,-1.1157577354,-2.3678875556$

$\mathrm{H}, 0.7217876951,-2.6754613525,-1.5723229672$

C,-3.5445375635,-1.1153447095,3.0713245809

$\mathrm{H},-3.8987074412,-2.1451945078,2.9478719815$

$\mathrm{H},-3.3378307816,-0.9599952316,4.1358821502$

$\mathrm{H},-4.3595671765,-0.4462819553,2.7808885355$

C, $0.5472525269,3.369499279,-0.5430196341$

$\mathrm{H}, 1.4622726724,3.5174121192,-1.1266416703$

$\mathrm{H},-0.2195429229,3.9979292933,-1.0077901286$

$\mathrm{H}, 0.7058931697,3.7258995289,0.4771990881$

C,-3.2514153778,2.1009967362,0.6590826458

$\mathrm{H},-3.3731686939,2.2423997696,-0.4200332072$

$\mathrm{H},-4.1432291525,1.6049308966,1.052054191$

$\mathrm{H},-3.193937512,3.0946541004,1.1173823638$

C,-2.9808857528,-0.4733793321,-3.2504228539

$\mathrm{H},-3.8211130686,-0.5331726183,-2.5503708725$

$\mathrm{H},-2.2119217091,0.1620864509,-2.7937784258$

$\mathrm{H},-3.3342509121,0.0196337139,-4.1627115374$

$\mathrm{H},-1.6671695361,-3.8798035434,-4.0306395424$

Zero-point correction=

Thermal correction to Energy=

Thermal correction to Enthalpy=

Thermal correction to Gibbs Free Energy $=0.423913$

Sum of electronic and zero-point Energies=

Sum of electronic and thermal Energies=

Sum of electronic and thermal Enthalpies=

Sum of electronic and thermal Free Energies=
0.392354 (Hartree/Particle)

0.422969

0.423913

0.327483

$-3313.415889$

$-3313.385273$

$-3313.384329$

$-3313.480759$ 
Table S9. Cartesian coordinates and energy values for the 16-Me.

Ge,-0.028260164,1.7186533609,-0.8854326089

$\mathrm{Si},-2.2013659338,-0.9251316286,1.0450162553$

$\mathrm{Si},-1.5797241591,1.305617575,0.867969327$

$\mathrm{N}, 2.3372069336,0.397042052,0.6250115199$

$\mathrm{N}, 1.7093952882,-0.7265087886,-1.1039587806$

C,-2.3420697266,-1.6996418405,-0.7756243194

C, $-1.8873253083,-2.9222933587,-0.8464384535$

C, $1.4330323283,0.3943238749,-0.3888962339$

C,3.1762464116,-0.7134494392,0.5525337264

$\mathrm{C}, 2.7696897033,-1.431028021,-0.5381406786$

C,4.2698130524,-0.9738952162,1.5345517501

C,3.2769877902,-2.7175219753,-1.0994647679

$\mathrm{H}, 3.8832429433,-1.1208264073,2.5497521545$

$\mathrm{H}, 4.9910213955,-0.1497080611,1.5704131177$

$\mathrm{H}, 4.8157254073,-1.8780645457,1.2586206791$

$\mathrm{H}, 4.0727551136,-3.1173113571,-0.4681560192$

$\mathrm{H}, 3.6866672315,-2.592723863,-2.1084754628$

$\mathrm{H}, 2.4852351521,-3.4721895636,-1.1554621897$

C,-0.8739555072,-1.8825111361,2.0121204164

$\mathrm{H}, 0.0979230218,-1.8470883755,1.5093463366$

$\mathrm{H},-0.754679363,-1.443203676,3.0082679894$

$\mathrm{H},-1.1495757047,-2.9349939705,2.1392160624$

C,2.3814123764,1.4120655703,1.6708496035

$\mathrm{H}, 3.051822165,2.2309846416,1.3953915388$

$\mathrm{H}, 1.3701513734,1.7970675599,1.809854524$

$\mathrm{H}, 2.7278787211,0.9613845034,2.6015682983$

C, $0.9661986117,-1.1728706389,-2.2831336364$

$\mathrm{H}, 0.1773732443,-1.8767666318,-1.9994113765$

$\mathrm{H}, 0.5064533594,-0.2962878757,-2.7442473538$

$\mathrm{H}, 1.6553863606,-1.6412056512,-2.9873918371$

C,-3.8604261741,-1.1756108092,1.9501614153

$\mathrm{H},-4.1372046061,-2.2356325644,1.9266842523$

$\mathrm{H},-3.7991529871,-0.8649468852,2.9979725903$

$\mathrm{H},-4.6700780166,-0.6112127916,1.4771927729$

C, $0.8818454157,3.4866818278,-0.5880191782$

$\mathrm{H}, 1.7799790701,3.5262973057,-1.2117760573$

$\mathrm{H}, 0.1935754731,4.2453994484,-0.9715042626$

$\mathrm{H}, 1.1443423029,3.7482513987,0.4395776852$

C,-3.0656981056,2.497555202,0.5703235841

$\mathrm{H},-3.1831205123,2.7383914273,-0.4912283793$

$\mathrm{H},-4.0053524089,2.0788044141,0.9424196242$

$\mathrm{H},-2.8912470301,3.4398430545,1.103074948$

C,-3.0268149653,-0.895599499,-1.8563978571

$\mathrm{H},-4.0027526747,-0.5252743538,-1.520701989$

$\mathrm{H},-2.422920915,-0.0202394616,-2.1247929984$

$\mathrm{H},-3.1763631657,-1.5128980537,-2.7479194621$

$\mathrm{H},-1.3277478181,-3.5168217764,-0.1262932693$

Zero-point correction=

Thermal correction to Energy=

Thermal correction to Enthalpy=

Thermal correction to Gibbs Free Energy=

Sum of electronic and zero-point Energies=

Sum of electronic and thermal Energies=

Sum of electronic and thermal Enthalpies=

Sum of electronic and thermal Free Energies=
0.393102 (Hartree/Particle)

0.422061

0.423006

0.334083

$-3313.402442$

$-3313.373483$

$-3313.372539$

$-3313.461462$ 
Table S10. Cartesian coordinates and energy values for the 17-Me.

Ge,-0.1308487777, 1.8337057435,1.051525349

Si,2.0332544529,-1.1377876276,-1.296222609

Si, $1.8080266999,0.8258178243,0.0168702452$

$\mathrm{N},-2.3084240003,0.3098209413,-0.6033002806$

$\mathrm{N},-1.7995278343,-0.6374385018,1.2623808667$

C, $2.5020152952,-0.5367892385,1.1857387392$

C, $2.5376322778,-1.6758417802,0.4431789642$

C, $-1.4731889007,0.4178162029,0.4649191209$

C,-3.1551701508,-0.7944862027,-0.4775898399

C,-2.8238190932,-1.3996994343,0.7004370276

C,-4.1843491661,-1.154137833,-1.4972376934

C,-3.3757314182,-2.6241007672,1.351519002

$\mathrm{H},-3.735628553,-1.4140963809,-2.4633433687$

$\mathrm{H},-4.8920221993,-0.3353812317,-1.6700390567$

$\mathrm{H},-4.7588722126,-2.0197604014,-1.1617207933$

$\mathrm{H},-4.1359135172,-3.0799544997,0.7141494753$

$\mathrm{H},-3.845642373,-2.4020635584,2.3168744675$

$\mathrm{H},-2.5977842967,-3.3753355016,1.5275896856$

C, $0.517228807,-2.0366428144,-2.0218839558$

$\mathrm{H},-0.3409511205,-1.9883506158,-1.3441094991$

$\mathrm{H}, 0.2193360572,-1.5881720042,-2.9769811786$

$\mathrm{H}, 0.7374473885,-3.0940512662,-2.2118013456$

C,-2.2315179045,1.1759404147,-1.771688508

$\mathrm{H},-2.7847936516,2.1056099275,-1.6169859339$

$\mathrm{H},-1.1839355344,1.4122704225,-1.9526740153$

$\mathrm{H},-2.641372694,0.6553962843,-2.6374027607$

C, $-1.118833328,-0.9444064602,2.5157622347$

$\mathrm{H},-0.2239453526,-1.5445381243,2.3313000258$

$\mathrm{H},-0.8203635242,0.0025736081,2.9767071828$

$\mathrm{H},-1.7960891734,-1.4854612875,3.1777220528$

C,3.4301907972,-1.2717648469,-2.5828716175

$\mathrm{H}, 3.6690507904,-2.318944998,-2.804624289$

$\mathrm{H}, 3.1448527168,-0.7909610663,-3.525520303$

$\mathrm{H}, 4.3444720957,-0.7887161311,-2.2246741313$

C,- $-0.5421323683,3.41005001,-0.1485393764$

$\mathrm{H},-1.5756577081,3.7347049863,0.0116586654$

$\mathrm{H}, 0.0933959611,4.2124019391,0.2405037398$

$\mathrm{H},-0.3539742994,3.3294270827,-1.222195195$

C,3.0950571312,2.2103870274,-0.2968344599

$\mathrm{H}, 3.1652339127,2.8787297298,0.5687706903$

$\mathrm{H}, 4.0875683937,1.791019024,-0.4913501827$

$\mathrm{H}, 2.8094105046,2.8218378267,-1.1596664094$

C,2.8685175852,-0.4104745295,2.6400614615

$\mathrm{H}, 3.7778726857,0.194193938,2.7558764255$

$\mathrm{H}, 2.0805554928,0.1229679868,3.1865939215$

$\mathrm{H}, 3.0479389594,-1.3805025349,3.1205535667$

$\mathrm{H}, 2.8466351469,-2.6404882816,0.8612348926$

Zero-point correction $=$

Thermal correction to Energy=

Thermal correction to Enthalpy=

Thermal correction to Gibbs Free Energy=

Sum of electronic and zero-point Energies=

Sum of electronic and thermal Energies=

Sum of electronic and thermal Enthalpies=

Sum of electronic and thermal Free Energies=
0.395682 (Hartree/Particle)

$$
0.423671
$$

0.424616

0.337956

$-3313.507159$

$-3313.479170$

$-3313.478225$

$-3313.564885$ 
Table S11. Cartesian coordinates and energy values for the 18-Me.

Ge, $0.5094900095,-0.0476584754,-0.5598690058$

Si, $1.4696571546,1.6034924851,0.8544137593$

Si,2.0429887406,-1.8427553977,0.15900055

$\mathrm{N},-2.0315291935,-0.907094564,0.7778397148$

$\mathrm{N},-2.492628105,0.383130565,-0.8857952999$

C,3.3912908029,-0.4369441513,0.3746680619

C,3.1733563415, $0.8538634201,0.7535118387$

C, $-1.4582759476,-0.1930312725,-0.2216718918$

C,-3.4190139972,-0.7841212237, 0.7443003104

C,-3.7147719438, $0.0281343562,-0.3133077822$

C,-4.3107172337,-1.462734588, 1.7298340397

C,-5.0325585171,0.4957583892,-0.8359671092

$\mathrm{H},-4.0768102487,-1.1669414281,2.758348947$

$\mathrm{H},-4.2257814898,-2.5538145128,1.6733217233$

$\mathrm{H},-5.353392766,-1.2023043513,1.5389672579$

$\mathrm{H},-5.8424673353,0.0554807708,-0.2514474869$

$\mathrm{H},-5.1860230332,0.2045185315,-1.8811005653$

$\mathrm{H},-5.1399144035,1.5849428452,-0.7735069279$

C, $1.467465758,3.4095202552,0.251109752$

$\mathrm{H}, 1.8750225009,3.5007438133,-0.760367083$

$\mathrm{H}, 0.4589145251,3.8387148818,0.2539823909$

$\mathrm{H}, 2.0853657288,4.0252306014,0.9164678131$

C,-1.3030159576,-1.6287108091,1.8233512964

$\mathrm{H},-1.8225827572,-2.5611989475,2.0485184741$

$\mathrm{H},-0.2895784769,-1.8599660456,1.4754462602$

$\mathrm{H},-1.2464565531,-1.0173844235,2.7285217544$

C,-2.3221173609,1.3176523496,-1.9942639469

$\mathrm{H},-3.2756579771,1.7977855955,-2.2083061015$

$\mathrm{H},-1.5914728605,2.0767027407,-1.7112024655$

$\mathrm{H},-1.9700818983,0.801530808,-2.8889916342$

C, $0.7445102613,1.6133354947,2.617420772$

$\mathrm{H}, 1.3115300358,2.3127279197,3.2432236619$

$\mathrm{H},-0.3041940157,1.9328320208,2.6373034013$

$\mathrm{H}, 0.8173227828,0.6235927441,3.0783441678$

C, $0.8144528005,0.5053425232,-2.4346614627$

$\mathrm{H}, 0.4780359594,1.506618957,-2.7075278874$

$\mathrm{H}, 1.906612935,0.4793279291,-2.4752502458$

$\mathrm{H}, 0.431981431,-0.2308907375,-3.1449567078$

C,2.5186986709,-2.5596951287,-1.6025241473

$\mathrm{H}, 2.7603526032,-1.8188848727,-2.373355177$

$\mathrm{H}, 3.3843977228,-3.2257935129,-1.4981056396$

$\mathrm{H}, 1.6977105432,-3.1807906234,-1.9811921359$

C, $4.8225964881,-0.9259067655,0.2419321394$

$\mathrm{H}, 4.9974803238,-1.8210601253,0.8525179907$

$\mathrm{H}, 5.0375210031,-1.2176491618,-0.7942703576$

$\mathrm{H}, 5.5544143839,-0.1633850847,0.5362551769$

$\mathrm{H}, 4.0471872251,1.4791044359,0.9771205373$

Zero-point correction $=$

Thermal correction to Energy=

Thermal correction to Enthalpy=

Thermal correction to Gibbs Free Energy=

Sum of electronic and zero-point Energies=

Sum of electronic and thermal Energies=

Sum of electronic and thermal Enthalpies=

Sum of electronic and thermal Free Energies=
0.396446 (Hartree/Particle)

0.423874

0.424818

0.340365

$-3313.511532$

$-3313.484104$

$-3313.483160$

$-3313.567613$ 
Table S12. Cartesian coordinates and energy values for the 19-Me.

$\mathrm{Ge},-1.1782394931,-1.4910652767,-0.04811376$

$\mathrm{Si},-2.0444842565,-0.5855400131,2.0411884035$

Si,-0.5061415053,0.7945032429,-0.3955892156

$\mathrm{N}, 2.4274966523,0.7096386369,0.4402814896$

$\mathrm{N}, 2.0558859266,-0.1351535385,-1.5088767592$

C,-1.5718515575,1.2684548235,2.1093770676

$\mathrm{H},-1.8713365153,1.8763360862,2.9750022559$

C,- $-0.9034278037,1.8929787994,1.1122015962$

C, $1.4466518375,0.5211220828,-0.4821775199$

C, $3.6378701206,0.172670429,0.0025932273$

C,3.4011505492,-0.3650829883,-1.2287500772

$\mathrm{C}, 4.886729481,0.2337271535,0.8172492724$

C,4.3239061267,-1.0802153202,-2.1589674043

$\mathrm{H}, 4.7987916865,-0.3435465926,1.7448994476$

H,5.145647894,1.2624355263,1.0915142269

$\mathrm{H}, 5.7252755478,-0.1787355685,0.2533432833$

$\mathrm{H}, 5.3352058627,-1.0866075078,-1.7482039892$

$\mathrm{H}, 4.3699308737,-0.598090841,-3.1417400202$

$\mathrm{H}, 4.0249531808,-2.1233699974,-2.3104568498$

C,- $-0.6116488929,3.3811516146,1.1266151127$

$\mathrm{H},-0.9267035074,3.8535909587,2.0644013404$

$\mathrm{H}, 0.4557200829,3.5975431068,0.9821843349$

$\mathrm{H},-1.1329997764,3.889719899,0.3058274379$

C,-3.9389071173,-0.6624771677,2.2732633716

$\mathrm{H},-4.4626111127,-0.1363981357,1.4688013015$

$\mathrm{H},-4.2881019147,-1.7010486464,2.2719763335$

$\mathrm{H},-4.2403915638,-0.2114357157,3.2266612384$

C,2.2767255136,1.3512661584,1.7434330652

$\mathrm{H}, 2.7465214399,2.3393161183,1.7424063368$

$\mathrm{H}, 1.218583983,1.4452181407,1.9755888991$

$\mathrm{H}, 2.7494251234,0.7321304603,2.5085648872$

C, $1.3528810659,-0.6764432612,-2.6708361861$

$\mathrm{H}, 2.0641831796,-1.2119927939,-3.2967776854$

$\mathrm{H}, 0.5735493651,-1.3677141235,-2.325049338$

$\mathrm{H}, 0.9053228637,0.1267055991,-3.25619238$

C,-1.2844157033,-1.3838581409,3.6039541414

$\mathrm{H},-1.5794772114,-0.856114242,4.5193139873$

$\mathrm{H},-1.6135171375,-2.4249452509,3.697322444$

$\mathrm{H},-0.1900632285,-1.3951559256,3.5511250359$

C,-2.8840209782,-1.5287981092,-1.1613327711

$\mathrm{H},-3.4477613863,-2.4125958883,-0.8474313474$

$\mathrm{H},-3.5384716859,-0.6573353595,-1.092671484$

$\mathrm{H},-2.6009381761,-1.6909947963,-2.2057124597$

C,- $-0.897886363,1.8660955284,-1.9366856622$

$\mathrm{H},-0.2523284477,2.7461515119,-2.0334212146$

$\mathrm{H},-0.8699125961,1.310951003,-2.8779654398$

$\mathrm{H},-1.9271594263,2.223748321,-1.8170409746$

Zero-point correction $=$

Thermal correction to Energy=

Thermal correction to Enthalpy=

Thermal correction to Gibbs Free Energy=

Sum of electronic and zero-point Energies=

Sum of electronic and thermal Energies=

Sum of electronic and thermal Enthalpies=

Sum of electronic and thermal Free Energies=
0.396578 (Hartree/Particle)

0.424125

0.425069

0.339911

$-3313.517420$

$-3313.489873$

$-3313.488929$

$-3313.574087$ 
Table S13. Cartesian coordinates and energy values for the 20-Me.

Ge, $1.0956342576,-1.1597133208,0.8107110376$

Si, $0.45938529,1.0432865076,1.0925995522$

Si,-1.2638805791,-0.4575637745, -0.2741553897

C,-2.7940543185,-0.7444504698,0.8124153002

$\mathrm{H},-2.7716604108,-1.7477165719,1.2496417386$

$\mathrm{H},-2.8552765058,-0.0226161844,1.6320242801$

$\mathrm{H},-3.7105451948,-0.6563272611,0.2171564059$

C,-1.2627096933,-1.7456428611,-1.6686085389

$\mathrm{H},-2.2279803777,-1.7207069811,-2.1894921771$

$\mathrm{H},-0.4742793129,-1.5460641333,-2.3980512664$

$\mathrm{H},-1.1160106244,-2.7562470219,-1.2754479054$

C,- $-0.1918365957,1.6549744655,2.7699515358$

$\mathrm{H},-1.2142820374,2.0413510541,2.7122247449$

$\mathrm{H},-0.1483060194,0.8460085561,3.5052020082$

$\mathrm{H}, 0.4492315523,2.4670530961,3.1366424127$

C, $2.0277361946,-1.0431961216,-0.9835422462$

$\mathrm{H}, 3.0849964207,-0.8937854435,-0.7404847259$

$\mathrm{H}, 1.937034538,-2.0135831601,-1.4788408749$

$\mathrm{H}, 1.7100597666,-0.2533168795,-1.6668294386$

C,- $0.3002932342,2.110700877,-0.2836721879$

C, $-1.1800393597,1.3178439828,-0.942455225$

$\mathrm{H},-1.797269726,1.7237531274,-1.7526517133$

C,- $-0.0682792261,3.5741529694,-0.5446458472$

$\mathrm{H},-0.704391217,3.9514553449,-1.3529192823$

$\mathrm{H},-0.2705710184,4.1687713436,0.3553735404$

$\mathrm{H}, 0.9772504313,3.7666318599,-0.8154427377$

Zero-point correction=

Thermal correction to Energy=

Thermal correction to Enthalpy=

Thermal correction to Gibbs Free Energy=

Sum of electronic and zero-point Energies=

Sum of electronic and thermal Energies=

Sum of electronic and thermal Enthalpies=

Sum of electronic and thermal Free Energies=
0.209623 (Hartree/Particle)

0.226460

0.227404

0.165803

$-2930.171315$

$-2930.154478$

$-2930.153534$

$-2930.215135$

Table S14. Cartesian coordinates and energy values for the 21-Me.

Ge,-0.5367812293,-0.9671128558,0.6332211025

$\mathrm{Si}, 1.4658407233,-0.561047889,-0.2990310262$

$\mathrm{Si},-0.989229766,1.3651817612,0.2312403048$

C, $-1.9727955237,2.2520094676,1.5893548445$

$\mathrm{H},-3.0073550083,1.8937165065,1.6206359684$

$\mathrm{H},-1.5322251317,2.082244641,2.5764991267$

$\mathrm{H},-2.0017619935,3.3335148844,1.4100127471$

C, $-1.8559814896,1.6650941478,-1.43483133$

$\mathrm{H},-2.0380906279,2.7344636083,-1.5911085339$

$\mathrm{H},-1.2479090153,1.29653073,-2.2656073183$

$\mathrm{H},-2.823742678,1.1536699531,-1.4686793246$

C,2.9959227527,-1.5697505334,-0.7809361703

$\mathrm{H}, 3.7587921062,-1.5249752502,0.0045711983$

$\mathrm{H}, 2.7468710471,-2.6188669709,-0.95840238$

$\mathrm{H}, 3.4409848677,-1.1619881053,-1.6958765225$

C,-1.6695781947,-2.218684312,-0.4159574712

$\mathrm{H},-1.2120101768,-3.2086517003,-0.4584361339$

$\mathrm{H},-2.6462113724,-2.3145730392,0.0625829903$

$\mathrm{H},-1.7967343937,-1.8249475513,-1.42465996$ 
C, $1.8621601,1.2552131582,0.0043994725$

C, $0.7785913748,2.0472027355,0.2133500333$

$\mathrm{H}, 0.9563469446,3.1003532776,0.4613965396$

C,3.2780713933, 1.7868370638,0.0065403646

$\mathrm{H}, 3.3042681942,2.8707125413,0.162090019$

$\mathrm{H}, 3.8799677404,1.3169678039,0.7947000767$

H,3.7872663566, 1.5699909272,-0.9409256175

Zero-point correction=

Thermal correction to Energy=

0.210321 (Hartree/Particle)

Thermal correction to Enthalpy=

0.227002

Thermal correction to Gibbs Free Energy=

0.227947

Sum of electronic and zero-point Energies=

0.166621

Sum of electronic and thermal Energies=

$-2930.194347$

Sum of electronic and thermal Enthalpies=

$-2930.177666$

Sum of electronic and thermal Free Energies=

$-2930.176722$

$-2930.238047$ 
Table S15. Cartesian coordinates and energy values for the 22-Me.

$\mathrm{Ge}, 0.446159182,-0.0959352446,1.5874379578$

Si, $0.3770218723,2.5041526523,-1.592127434$

Si, $0.7531750321,2.0377165395,0.518421793$

$\mathrm{N},-2.5229263349,-0.5774148381,0.7020119659$

$\mathrm{N},-1.0374646593,-1.4513308641,-0.5911188163$

C, $4.7672510905,-2.4900956331,-0.3831364675$

C,3.8971451798,-1.8513219867,0.1606136661

C, $-1.1811218059,-0.6768600218,0.5159917021$

C,-3.2204448661,-1.2921891206,-0.2739468087

C,-2.2767537829,-1.8387845629,-1.0981776529

C,-4.7106563658,-1.3692237389,-0.3152981999

C,-2.4336916375,-2.6877881636,-2.3158437964

$\mathrm{H},-5.1705442789,-0.3876079121,-0.4805675428$

$\mathrm{H},-5.1242251842,-1.7757589794,0.6145682706$

$\mathrm{H},-5.0316753431,-2.0201519735,-1.1308497362$

$\mathrm{H},-3.491887137,-2.8713848838,-2.5116800179$

$\mathrm{H},-1.9450427151,-3.662104079,-2.2029482201$

$\mathrm{H},-2.013385654,-2.2056172153,-3.2056899006$

C,-1.2598183762,2.0010812487,-2.4351981265

$\mathrm{H},-1.7587178156,1.2052335209,-1.8744959429$

$\mathrm{H},-1.9502322778,2.8488245229,-2.5103832095$

$\mathrm{H},-1.0807235312,1.6338175796,-3.4524061994$

C,-3.131924415,0.24907019,1.7364525678

$\mathrm{H},-3.2619507337,-0.309692366,2.6675281992$

$\mathrm{H},-2.4797556098,1.1019118531,1.9175121069$

$\mathrm{H},-4.10461254,0.6034266453,1.3935358067$

C, $0.2674044059,-1.7853875105,-1.1601002753$

$\mathrm{H}, 0.8092100674,-0.8649631501,-1.3958003802$

$\mathrm{H}, 0.8517583744,-2.3540636269,-0.433382921$

$\mathrm{H}, 0.1257299913,-2.3708942001,-2.067391739$

C, $1.0870055366,4.0637474652,-2.4261969543$

$\mathrm{H}, 1.3616959166,3.8574703204,-3.4669126669$

$\mathrm{H}, 0.3578183998,4.8812937015,-2.4296325307$

$\mathrm{H}, 1.9847987468,4.4107472593,-1.9072840292$

C,- $0.2813079182,0.3624075558,3.4136226421$

$\mathrm{H},-0.7601481347,-0.5227212326,3.8458869276$

$\mathrm{H}, 0.6177694545,0.5495797042,4.0098148323$

$\mathrm{H},-0.9441961454,1.223750045,3.5213601261$

C,2.3707101703,2.8202583494,1.2003877994

$\mathrm{H}, 2.1410039316,3.4665755686,2.0549003023$

$\mathrm{H}, 3.0441874527,2.0321992819,1.5525216227$

$\mathrm{H}, 2.8968083535,3.4248870427,0.4568083591$

C,5.8222359109,-3.2573483442,-1.0381179196

$\mathrm{H}, 6.7502211458,-3.231372596,-0.4560277387$

$\mathrm{H}, 5.5357458428,-4.3084446683,-1.1570364604$

$\mathrm{H}, 6.0447577657,-2.8585584817,-2.0342303698$

$\mathrm{H}, 3.120818439,-1.2939696525,0.6460394091$

Zero-point correction=

Thermal correction to Energy=

Thermal correction to Enthalpy=

Thermal correction to Gibbs Free Energy=

Sum of electronic and zero-point Energies=

Sum of electronic and thermal Energies=

Sum of electronic and thermal Enthalpies=

Sum of electronic and thermal Free Energies=
0.391793 (Hartree/Particle) 0.422936

0.423880

0.323312

$-3313.416262$

$-3313.385120$

$-3313.384175$

$-3313.484744$ 
Table S16. Cartesian coordinates and energy values for the 23-Me.

Ge,0.1488616972,-1.249915812,-0.7411002058

$\mathrm{Si}, 2.1794055639,0.300904191,1.6600048924$

Si, $1.2624874225,-1.8762461085,1.3179529622$

$\mathrm{N},-2.3996529272,-0.2279853544,0.4710716454$

$\mathrm{N},-1.4825278569,1.3172205858,-0.7195214774$

C, $3.1756818693,1.6003407359,-0.9473116318$

C,2.7714690564,1.1621647445,0.1190585969

C,-1.3520156929,0.0242213732,-0.3456699687

C,-3.2067053146,0.9001580412,0.6020085123

C,-2.6226658225, $1.8831308703,-0.1489012824$

C,-4.4426042229,0.9208270222, 1.4383977153

C,-3.0312199814,3.3019104505,-0.3694127395

$\mathrm{H},-4.2199844488,0.7648337472,2.5001205629$

$\mathrm{H},-5.1554766139,0.1480261529,1.1308310115$

$\mathrm{H},-4.9426672527,1.8866700169,1.3469122485$

$\mathrm{H},-3.9671132528,3.5068803315,0.1536354681$

$\mathrm{H},-3.1918797377,3.5227922054,-1.4304435109$

$\mathrm{H},-2.2824135424,4.0060554602,0.0101865457$

C, $0.9083981312,1.4798794233,2.4658709467$

$\mathrm{H}, 0.0196978056,1.6112143752,1.8387229547$

$\mathrm{H}, 0.5741536403,1.0624925912,3.4223228069$

$\mathrm{H}, 1.3363806797,2.4701039167,2.6572199041$

C,-2.6449253137,-1.5196637524,1.1154277137

$\mathrm{H},-3.2309758904,-2.1699455575,0.4601668026$

$\mathrm{H},-1.6822134259,-1.9850079616,1.3486994284$

$\mathrm{H},-3.1895704716,-1.3586935993,2.0454083504$

C, $-0.5269018382,2.0144897362,-1.584307469$

$\mathrm{H}, 0.4782828669,1.9324492019,-1.1653554573$

$\mathrm{H},-0.5362244221,1.5697886183,-2.5815696643$

$\mathrm{H},-0.8116481565,3.063147588,-1.6516853402$

C,3.6840646184,0.2374739404,2.8294440094

$\mathrm{H}, 4.0720221853,1.238276011,3.0491438617$

$\mathrm{H}, 3.4042176402,-0.2366042084,3.7771088768$

$\mathrm{H}, 4.49750203,-0.3504752271,2.3925460594$

C,- $0.7575365909,-2.7214342659,-1.702026737$

$\mathrm{H},-1.4704603046,-2.3547502492,-2.4448564293$

$\mathrm{H}, 0.0230858798,-3.2768800321,-2.2292961471$

$\mathrm{H},-1.2536157058,-3.4061048112,-1.0105259602$

C,2.7441346165,-2.7496235153,0.3507938421

$\mathrm{H}, 2.3968357628,-3.6947723908,-0.0835102085$

$\mathrm{H}, 3.1842783306,-2.1563333659,-0.4587759497$

$\mathrm{H}, 3.5442637465,-3.0000360813,1.0574006089$

C,3.6850716882,2.1224220512,-2.2123367784

$\mathrm{H}, 4.7759473643,2.0281202293,-2.265366198$

$\mathrm{H}, 3.2673937791,1.5780617806,-3.0677083006$

$\mathrm{H}, 3.4430147919,3.1844593235,-2.3408628463$

$\mathrm{H}, 0.8429456199,-0.4828904226,-1.9013960247$

Zero-point correction $=$

Thermal correction to Energy=

Thermal correction to Enthalpy=

Thermal correction to Gibbs Free Energy=

Sum of electronic and zero-point Energies=

Sum of electronic and thermal Energies=

Sum of electronic and thermal Enthalpies=

Sum of electronic and thermal Free Energies=
0.391683 (Hartree/Particle)

0.421075

0.422019

0.330468

$-3313.475373$

$-3313.445981$

$-3313.445037$

$-3313.536588$ 
Table S17. Cartesian coordinates and energy values for the 24-Me.

Ge,-0.6674688576,-1.7456511922,0.0765340296

$\mathrm{Si},-2.1045707896,1.5018915905,-0.4384193684$

Si,-2.4420739818,-0.7312427812,-1.2048423278

$\mathrm{N}, 2.0314239195,-0.946082696,-1.0348092232$

$\mathrm{N}, 1.778847109,-0.1526873999,0.9528292535$

C,-2.2737231268,1.5868214405,2.6331301427

C,-2.1637798393,1.5680410958,1.4178769362

C, $1.1975608194,-0.9607360369,0.0333308757$

C,3.14095571,-0.1374421859,-0.7912591913

$\mathrm{C}, 2.9778421532,0.3715166027,0.4669210442$

C,4.2303239086, $0.0676384127,-1.7904777263$

C,3.8376478202,1.3105268265,1.2462991373

$\mathrm{H}, 3.8641815539,0.5562315,-2.7008785741$

$\mathrm{H}, 4.697919129,-0.8783444829,-2.0857414422$

$\mathrm{H}, 5.0114234325,0.7035676296,-1.3700647758$

$\mathrm{H}, 4.7197054333,1.5811386434,0.6628969716$

$\mathrm{H}, 4.1877748673,0.8659097705,2.1847527703$

$\mathrm{H}, 3.3084894909,2.2378905391,1.4917212641$

C,- $0.4840787414,2.3842262333,-0.9523325927$

$\mathrm{H}, 0.3983461471,1.8702306292,-0.5578128719$

$\mathrm{H},-0.3960767106,2.3922559073,-2.0450181744$

$\mathrm{H},-0.4580281093,3.4244436355,-0.6078121496$

C, $1.8049727476,-1.7145870329,-2.2547399363$

$\mathrm{H}, 2.2231082169,-2.7211887595,-2.1644531091$

$\mathrm{H}, 0.73326276,-1.7788788384,-2.4377374788$

$\mathrm{H}, 2.2708953522,-1.2055120636,-3.0983511628$

C, $1.2000586562,0.1284140406,2.2684717715$

$\mathrm{H}, 0.2280622337,0.6146668798,2.1566372221$

$\mathrm{H}, 1.0701894654,-0.8053997383,2.8186313177$

$\mathrm{H}, 1.8745550177,0.7818138007,2.8193601421$

C,-3.5252343935,2.6201155619,-1.0467948072

$\mathrm{H},-3.4191890637,3.6496028586,-0.6870864774$

$\mathrm{H},-3.5487764849,2.6455178587,-2.1427159777$

$\mathrm{H},-4.4913865107,2.23597883,-0.7056217802$

C,- $0.3582552462,-3.6638755202,-0.2791975295$

$\mathrm{H}, 0.5405299527,-4.0416889877,0.2149990205$

$\mathrm{H},-1.2224872102,-4.1917427873,0.1322993339$

$\mathrm{H},-0.313344252,-3.8773057832,-1.3497166274$

C,-1.6449541881,-0.5959967658,-3.0053845823

$\mathrm{H},-1.4941372106,-1.5927248676,-3.4397247091$

$\mathrm{H},-2.3759622998,-0.094410337,-3.6515132961$

$\mathrm{H},-0.7059955222,-0.0369657795,-3.0957051742$

C,-2.4270180518,1.5942584304,4.0852553462

$\mathrm{H},-3.4081191539,1.9878619472,4.375047362$

$\mathrm{H},-2.3432206439,0.5834851554,4.5017131112$

$\mathrm{H},-1.6677259647,2.2196873462,4.5699463564$

$\mathrm{H},-0.8993435437,-1.7020051301,1.6047586571$

Zero-point correction $=$

Thermal correction to Energy=

Thermal correction to Enthalpy=

0.391743 (Hartree/Particle)

0.421129

0.422074

Thermal correction to Gibbs Free Energy $=\quad 0.329428$

Sum of electronic and zero-point Energies=

Sum of electronic and thermal Energies=

Sum of electronic and thermal Enthalpies=

$-3313.471805$

$-3313.442418$

$-3313.441474$

Sum of electronic and thermal Free Energies $=\quad-3313.534120$ 
Table S18. Cartesian coordinates and energy values for the 25-Me.

Ge,-0.4784994514,-1.6916260256,0.1713572967

$\mathrm{Si},-1.385647491,1.9621994426,-0.7791129729$

$\mathrm{Si},-2.3006835512,-0.1756793872,-0.3708645059$

$\mathrm{N}, 2.0928586979,-0.5744888693,-0.8682524799$

$\mathrm{N}, 1.8306794557,-0.0261208525,1.2028729176$

C,-2.1446256025, $0.7423863877,1.2941585449$

C, $-1.2541664951,1.7602601932,1.0714437848$

C, $1.2682794035,-0.7231606724,0.194812515$

C,3.1885845124,0.2160419016,-0.5318624466

$\mathrm{C}, 3.0140880873,0.5703717706,0.7789948764$

C, $4.2717204968,0.5627929788,-1.4983245569$

C,3.8464764591,1.4381281205,1.6629552355

$\mathrm{H}, 3.8976541893,1.1719588804,-2.3293257319$

$\mathrm{H}, 4.7456959998,-0.3297466737,-1.9214848415$

$\mathrm{H}, 5.0490100822,1.1406709185,-0.9954486986$

$\mathrm{H}, 4.7233452984,1.7962044198,1.120367627$

$\mathrm{H}, 4.2013408749,0.8997799319,2.5486826073$

$\mathrm{H}, 3.2844349199,2.3133048156,2.0052307754$

C, $0.1523116681,2.3465815625,-1.8512567254$

$\mathrm{H}, 1.0199183245,1.7453324491,-1.5661449949$

$\mathrm{H},-0.0480059202,2.1823786799,-2.9165586274$

$\mathrm{H}, 0.4291792375,3.4010505461,-1.7310542351$

C, $1.8056044797,-1.1049513634,-2.1982539052$

$\mathrm{H}, 2.0764939525,-2.1607636045,-2.2676500584$

$\mathrm{H}, 0.7396689115,-0.9842682433,-2.4000574472$

$\mathrm{H}, 2.3655363319,-0.5373552458,-2.9400481629$

C, $1.1972221284,0.2224713373,2.5070267884$

$\mathrm{H}, 0.45155869,1.0291805137,2.3768797619$

$\mathrm{H}, 0.6960776972,-0.6896089354,2.8303337929$

$\mathrm{H}, 1.9704191993,0.4853720964,3.228404399$

C,-2.6893836524,3.2331491514,-1.328341985

$\mathrm{H},-2.3674591447,4.2438397684,-1.0478172542$

$\mathrm{H},-2.846065935,3.219441747,-2.4129104254$

$\mathrm{H},-3.6488121044,3.0421420447,-0.8395410065$

C,- $-0.1123618073,-3.4302334217,-0.6916425061$

$\mathrm{H}, 0.8528552243,-3.8430904678,-0.3860968626$

$\mathrm{H},-0.8919795522,-4.1122382909,-0.3406465231$

$\mathrm{H},-0.1695287528,-3.386052542,-1.7815035977$

C,-3.5951197502,-1.4737649362,-1.0040349716

$\mathrm{H},-3.7760326827,-2.2504521205,-0.250042058$

$\mathrm{H},-4.5510328009,-0.989683543,-1.2304187915$

$\mathrm{H},-3.2394681604,-1.9721483051,-1.914485078$

C,-2.6524858087, $0.2755331128,2.6405391236$

$\mathrm{H},-3.7462086519,0.3526868124,2.7062973857$

$\mathrm{H},-2.4022507698,-0.7811498501,2.8229477734$

$\mathrm{H},-2.2217072179,0.885540835,3.4437977438$

$\mathrm{H},-0.5613630194,-2.0929530675,1.6662795009$

Zero-point correction $=$

Thermal correction to Energy=

Thermal correction to Enthalpy=

Thermal correction to Gibbs Free Energy=

Sum of electronic and zero-point Energies=

Sum of electronic and thermal Energies=

Sum of electronic and thermal Enthalpies=

Sum of electronic and thermal Free Energies=
0.391681 (Hartree/Particle)

0.419673

0.420617

0.334997

$-3313.425744$

$-3313.397752$

$-3313.396808$

$-3313.482428$ 
Table S19. Cartesian coordinates and energy values for the 26-Me.

Ge,-0.3189742353,-0.4528783251,-1.2921479032

$\mathrm{Si}, 3.4279384178,2.0341499663,-0.185340144$

Si, $1.7760452227,0.3715035391,0.0603395802$

$\mathrm{N},-2.5939453356,-1.3581737962,0.4398035858$

$\mathrm{N},-3.0591205327,0.5165436078,-0.5332108987$

$\mathrm{C}, 3.4242192042,-0.542675966,0.533173461$

C, $4.1367201493,0.565956669,0.6846176426$

C,-2.1626158317,-0.5138402227,-0.5413992518

C,-3.7427672871,-0.8623090211, 1.0607885756

C,-4.0333710677, $0.3239605369,0.450095741$

C,-4.4434262134,-1.5793996082,2.1662824846

C, $-5.146228157,1.288474316,0.6925159286$

$\mathrm{H},-3.8144829291,-1.6760420139,3.0591801081$

$\mathrm{H},-4.7540621705,-2.5873584459,1.8678010321$

$\mathrm{H},-5.3419440383,-1.0328556991,2.4585945388$

$\mathrm{H},-5.7940409916,0.9220719477,1.4910633778$

$\mathrm{H},-5.7701462687,1.4279365059,-0.198194262$

$\mathrm{H},-4.7764540386,2.2746081317,0.9966964933$

C,4.1869671662,2.3297247695,-1.8942319071

$\mathrm{H}, 4.2187849126,1.3993678616,-2.4675834385$

$\mathrm{H}, 3.608424568,3.0652817477,-2.4636436002$

$\mathrm{H}, 5.2116646804,2.7073629422,-1.8007451237$

C,-1.8634510972,-2.5601176232,0.8085150051

$\mathrm{H},-1.9160361156,-3.3095882348,0.0146950986$

$\mathrm{H},-0.8139451229,-2.2992522087,0.9787924106$

$\mathrm{H},-2.2786285684,-2.9786214323,1.7242179752$

C,-2.9187162825,1.6870924871,-1.3861303414

$\mathrm{H},-2.0231489288,2.2507307362,-1.1046728148$

$\mathrm{H},-2.8109852513,1.3751489465,-2.4273233045$

$\mathrm{H},-3.7963310359,2.3237360228,-1.2828890313$

C,3.3488660691,3.6846125776,0.7456692552

$\mathrm{H}, 4.3498789512,4.1173542367,0.8536212696$

$\mathrm{H}, 2.7302215055,4.4118282079,0.2078243216$

$\mathrm{H}, 2.9281018658,3.5552324915,1.7471441831$

C, $0.0423633679,-2.2306410477,-2.0821862724$

$\mathrm{H},-0.8343266706,-2.6646722526,-2.567572839$

$\mathrm{H}, 0.8072026916,-2.0718880774,-2.8470101179$

$\mathrm{H}, 0.4445019588,-2.9247473659,-1.3405147596$

$\mathrm{C}, 0.8378229608,0.7192024658,1.7194179817$

$\mathrm{H}, 0.4511162419,-0.2062642694,2.1645236598$

$\mathrm{H}, 1.5147860676,1.1775819274,2.447562995$

$\mathrm{H},-0.0180406933,1.3861043343,1.5654463916$

C,3.7275474855,-1.9936827428, 0.7714478031

$\mathrm{H}, 3.0562941908,-2.4267957187,1.5247012066$

$\mathrm{H}, 3.5867580619,-2.5746719557,-0.1485245673$

$\mathrm{H}, 4.7597024705,-2.1257828015,1.1125425206$

$\mathrm{H},-0.5987773464,0.4203088538,-2.5458570496$

Zero-point correction=

Thermal correction to Energy=

Thermal correction to Enthalpy=

Thermal correction to Gibbs Free Energy=

Sum of electronic and zero-point Energies=

Sum of electronic and thermal Energies=

Sum of electronic and thermal Enthalpies=

Sum of electronic and thermal Free Energies=
0.390850 (Hartree/Particle)

$$
0.419740
$$

0.420684

0.329498

$-3313.426155$

$-3313.397265$

$-3313.396320$

$-3313.487507$ 
Table S20. Cartesian coordinates and energy values for the 27-Me.

Ge, $0.1416629491,-1.0442741247,0.0433622496$

Si, $2.4375889775,2.0118611486,0.2611328601$

$\mathrm{Si}, 0.8970065501,0.8750547886,1.2678094283$

$\mathrm{N},-2.9814645082,-0.7034622805,0.454158317$

$\mathrm{N},-2.1613487863,0.363767305,-1.226337769$

C, $5.0277688385,-2.1064523999,-1.0464616649$

$\mathrm{C}, 6.1423846443,-1.966430426,-1.4915828202$

C, $-1.8059487809,-0.4185681293,-0.1685952675$

C,- $-4.0631673757,-0.1152415282,-0.2055840821$

C,-3.5403741696,0.571407398,-1.2628771969

C, $-5.4745202025,-0.2737114699,0.2534250225$

C,- $-4.2149219684,1.4004625302,-2.30475618$

$\mathrm{H},-5.6347832836,0.1526922383,1.2509608668$

$\mathrm{H},-5.7751482474,-1.3269556166,0.2937075104$

$\mathrm{H},-6.1542552904,0.2362910786,-0.4319672669$

$\mathrm{H},-5.2900494644,1.4356373077,-2.1181710763$

$\mathrm{H},-4.0694017752,0.9945053103,-3.3126010917$

$\mathrm{H},-3.846296837,2.4323945087,-2.3070013235$

C,3.0337200975,1.394321331,-1.4299104824

$\mathrm{H}, 2.4154834554,0.5317533667,-1.6982373215$

$\mathrm{H}, 2.9307238677,2.1572992216,-2.2093664236$

$\mathrm{H}, 4.0803386403,1.0724864687,-1.4102603939$

C,-3.0847792647,-1.4762939441,1.6833373754

$\mathrm{H},-3.3045188561,-2.5284057486,1.4767327796$

$\mathrm{H},-2.1348599299,-1.4066197581,2.205722319$

$\mathrm{H},-3.8769763398,-1.0654370852,2.3118054319$

C,-1.1955469006,0.9513102016,-2.149336586

$\mathrm{H},-0.6592773903,1.7724311408,-1.6644133948$

$\mathrm{H},-0.4659477564,0.1857427167,-2.4277401401$

$\mathrm{H},-1.7157439061,1.3137172522,-3.0356443865$

C, $3.3770089304,3.5344574345,0.9016333765$

$\mathrm{H}, 4.4450764096,3.31560418,1.0081432869$

$\mathrm{H}, 3.2763493518,4.3800065868,0.2123701915$

$\mathrm{H}, 2.9960055864,3.8457772432,1.8784129367$

C, $0.0546872282,-2.4611420763,1.4884547368$

$\mathrm{H},-0.5868773338,-3.2899723555,1.1718083158$

$\mathrm{H}, 1.0783348259,-2.848388099,1.5113976965$

$\mathrm{H},-0.2073949609,-2.1652834625,2.50734879$

C, $0.3292308346,1.5360325695,2.9792655754$

$\mathrm{H},-0.7282731574,1.8199842043,2.9689290141$

$\mathrm{H}, 0.4666694469,0.7779130403,3.7572561285$

$\mathrm{H}, 0.9145813139,2.4170927246,3.2599771398$

C,3.6774373229,-2.2545159109,-0.5146780808

$\mathrm{H}, 2.9261972445,-2.2741815208,-1.3113088681$

$\mathrm{H}, 3.5804963873,-3.1836472201,0.0583537597$

$\mathrm{H}, 3.4121944861,-1.421613382,0.1459155761$

$H, 7.1272650966,-1.8545167582,-1.8805428678$

Zero-point correction $=$

Thermal correction to Energy=

Thermal correction to Enthalpy=

Thermal correction to Gibbs Free Energy 0.423923

Sum of electronic and zero-point Energies=

Sum of electronic and thermal Energies=

Sum of electronic and thermal Enthalpies $=$

Sum of electronic and thermal Free Energies=
0.392304 (Hartree/Particle)

0.422978

0.423923

0.326401

$-3313.417955$

$-3313.387280$

$-3313.386336$

$-3313.483858$ 
Table S21. Cartesian coordinates and energy values for the 28-Me.

Ge, $0.5036653953,0.916376777,0.4371393098$

Si,2.379503178,-0.659061224,-1.4766153779

Si, $1.3934962204,1.5127898685,-1.7447577004$

$\mathrm{N},-2.3984589156,0.7984013711,-0.2444492823$

$\mathrm{N},-1.8277695146,-1.1486179893,0.4760902145$

C, $1.6964818027,-0.4098834756,1.2865287494$

$\mathrm{C}, 2.502631184,-1.0323517058,0.4015288728$

C,-1.3556331699,0.1062006382,0.2776614295

C,-3.5279162864,-0.0093136351,-0.36046281

C,-3.1659709576,-1.2450066214,0.0955855922

C,- $-4.8302528386,0.4774714943,-0.9032594135$

C,-3.9439954392,-2.5136677855,0.2050077805

$\mathrm{H},-4.7503260094,0.7739161665,-1.9552307762$

$\mathrm{H},-5.2104981038,1.3378536001,-0.3419649169$

$\mathrm{H},-5.5798888993,-0.3136590069,-0.8425416972$

$\mathrm{H},-4.9796876115,-2.3486324648,-0.0973515615$

$\mathrm{H},-3.9552368722,-2.8985258334,1.2308342606$

$\mathrm{H},-3.5345390592,-3.3005049222,-0.4388564526$

C, $1.2501883994,-2.0231608179,-2.2131257056$

$\mathrm{H}, 0.2230766018,-1.8960672273,-1.8507038041$

$\mathrm{H}, 1.2199644943,-1.9541853983,-3.3060247878$

$\mathrm{H}, 1.5822884631,-3.0340734359,-1.94760584$

C,-2.3257884265,2.2030233593,-0.6524930535

$\mathrm{H},-2.3739165925,2.8564029056,0.221634265$

$\mathrm{H},-1.3842522652,2.3649058544,-1.188883998$

$\mathrm{H},-3.1597390087,2.4254676176,-1.3163921874$

C,-1.0651778645,-2.2604103803,1.0382904059

$\mathrm{H},-1.2388720448,-3.160445275,0.4457489211$

$\mathrm{H},-0.0066332424,-2.0108536943,0.9993053247$

$\mathrm{H},-1.3686384658,-2.4467371663,2.0727896649$

C,4.1154082183,-0.9220404837,-2.2250242879

$\mathrm{H}, 4.4964400824,-1.932407964,-2.0320387772$

$\mathrm{H}, 4.0889992226,-0.7768049617,-3.3107739247$

$\mathrm{H}, 4.8374549367,-0.2077535383,-1.8155684869$

C, $0.2333761519,2.3579640447,1.7614577923$

$\mathrm{H},-0.3917026443,2.0653461937,2.6085885162$

$\mathrm{H}, 1.235363583,2.5931944865,2.1301062152$

$\mathrm{H},-0.171724478,3.2577523567,1.2937133893$

C,2.7906722525,2.5623016122,-0.7901465464

$\mathrm{H}, 2.3584443148,3.5121613517,-0.4533304651$

$\mathrm{H}, 3.2348670294,2.078830835,0.0896508574$

$\mathrm{H}, 3.6042374252,2.8056472486,-1.4833959699$

C, $1.7616784444,-0.6094640025,2.781505957$

$\mathrm{H}, 0.7889101437,-0.9041877326,3.1990647588$

$\mathrm{H}, 2.4993172973,-1.3682032769,3.0699100075$

$\mathrm{H}, 2.0310497814,0.329026018,3.2825898072$

$\mathrm{H}, 3.2394410878,-1.7484807806,0.7902567312$

Zero-point correction $=$

Thermal correction to Energy=

Thermal correction to Enthalpy=

Thermal correction to Gibbs Free Energy=

Sum of electronic and zero-point Energies=

Sum of electronic and thermal Energies=

Sum of electronic and thermal Enthalpies=

Sum of electronic and thermal Free Energies=
0.396063 (Hartree/Particle)

0.423745

0.424689

0.339788

$-3313.508634$

$-3313.480953$

$-3313.480009$

$-3313.564909$ 
Table S22. Cartesian coordinates and energy values for the 29-Me.

Ge, $0.3739442143,-1.2318074241,-0.4792306115$

Si, $2.9635279216,0.4081642028,0.4805943825$

Si, $1.5798403015,-1.194388923,1.6256157465$

$\mathrm{N},-2.4050355402,-0.6483584201,0.4707662716$

$\mathrm{N},-1.6435409853,1.0877244994,-0.5512903717$

C, $1.4703216114,-0.355435248,-1.8574667984$

$\mathrm{C}, 2.5569376112,0.284038476,-1.3808993359$

C, $-1.3604141859,-0.2081603827,-0.2754712701$

C,-3.3519222133,0.3588755939,0.6503805895

C,-2.8686974514,1.4615242286,0.0045339938

C,-4.612710104,0.1643908982,1.4246631647

C,-3.449765687,2.8293435941, -0.1361014142

$\mathrm{H},-4.4156215665,-0.058596197,2.4796709635$

$\mathrm{H},-5.2206140572,-0.6521761096,1.0192895623$

$\mathrm{H},-5.2178264752,1.0721338752,1.3917516056$

$\mathrm{H},-4.4183476938,2.8763528635,0.3648305491$

$\mathrm{H},-3.6088831977,3.102702798,-1.185396059$

$\mathrm{H},-2.8099120057,3.5953758591,0.3160560716$

C,2.7879109518,2.2534820591,0.9825816772

$\mathrm{H}, 1.7489248344,2.6018809716,0.9619973104$

$\mathrm{H}, 3.1540598186,2.4044126905,2.0046700851$

$\mathrm{H}, 3.3747584558,2.9042471019,0.322760342$

C,-2.486952868,-1.9907348109,1.0431332042

$\mathrm{H},-2.7654121978,-2.72074365,0.2798413196$

$\mathrm{H},-1.517527404,-2.2580200323,1.4690071047$

$\mathrm{H},-3.2318238728,-1.9977448602,1.8375050718$

C,- $0.7568341707,1.9734264551,-1.3044695159$

$\mathrm{H}, 0.2274412942,1.9947097334,-0.8379886533$

$\mathrm{H},-0.6518296251,1.6201292735,-2.3310841639$

$\mathrm{H},-1.1795238278,2.9764078887,-1.31482403$

C, $4.8320536156,0.0438962204,0.6329930906$

$\mathrm{H}, 5.4356453679,0.7276723186,0.0230362246$

$\mathrm{H}, 5.1584551666,0.1489257294,1.6741332981$

$\mathrm{H}, 5.0618666805,-0.980329959,0.3217979133$

C,- $-0.190217448,-2.9375104736,-1.2990633119$

$\mathrm{H},-0.8977496362,-2.8036250917,-2.1223654229$

$\mathrm{H}, 0.7231439865,-3.3880212473,-1.6983161134$

$\mathrm{H},-0.6015933852,-3.6333318884,-0.5649266046$

C, $0.3977578686,0.0096402832,2.6700662073$

$\mathrm{H}, 0.0253791465,0.9091119716,2.1662007342$

$\mathrm{H},-0.4709721818,-0.5393055948,3.0567711781$

$\mathrm{H}, 0.9591038998,0.33904125,3.5525238645$

C, $1.1759685344,-0.5129120673,-3.3303807963$

$\mathrm{H}, 0.165287782,-0.1635350991,-3.5851175417$

$\mathrm{H}, 1.892616432,0.0291651612,-3.9589878741$

$\mathrm{H}, 1.2050197111,-1.5704717412,-3.6223510638$

H,3.2458265741, $0.7274172233,-2.1134215736$

\section{Zero-point correction=}

Thermal correction to Energy=

Thermal correction to Enthalpy=

Thermal correction to Gibbs Free Energy=

Sum of electronic and zero-point Energies=

Sum of electronic and thermal Energies=

Sum of electronic and thermal Enthalpies=

Sum of electronic and thermal Free Energies=
0.395883 (Hartree/Particle)

0.422831

0.423775

0.340980

$-3313.505888$

$-3313.478940$

$-3313.477996$

$-3313.560791$ 
Table S23. Cartesian coordinates and energy values for the 30-Me.

Ge,-0.4567788437,-0.2525725752,-0.6946980084

$\mathrm{Si},-1.6293933578,1.5847962671,0.4507427535$

Si,-1.3225438215,-0.4089426876,1.5919473426

$\mathrm{N}, 2.350411123,-0.8720217653,0.1689929352$

$\mathrm{N}, 2.2830229723,1.1532879252,-0.5678534211$

$\mathrm{C}, 1.4853846832,0.0835811236,-0.2799807851$

C,3.6241825523,0.8742019722,-0.293690837

C,3.6674096902,-0.4046940958,0.1770748676

C,4.7109020551,1.8729005166,-0.5141216677

$\mathrm{H}, 4.5590898565,2.7807131914,0.0817794296$

$\mathrm{H}, 4.7776592328,2.179480286,-1.5647762634$

$\mathrm{H}, 5.6778933665,1.4514065724,-0.2323571704$

C,4.8228633837,-1.2301048937,0.6362143622

$\mathrm{H}, 5.7423378425,-0.6422762143,0.6000272754$

$\mathrm{H}, 4.9747780992,-2.1148667968,0.006314932$

$\mathrm{H}, 4.6934380133,-1.5773555977,1.6677819215$

C,-2.7581541855,-1.618132318,1.9254821731

$\mathrm{H},-3.2890890087,-1.3397356903,2.8429093957$

$\mathrm{H},-3.4746137842,-1.6088790839,1.0990063655$

$\mathrm{H},-2.3932356714,-2.6451755424,2.0452854941$

C,- $-0.2235019211,-0.3210474827,3.1562472998$

$\mathrm{H}, 0.5909566649,0.4001337371,3.0388791797$

$\mathrm{H},-0.8231245416,-0.0167861434,4.0219016852$

$\mathrm{H}, 0.2206319704,-1.2950203823,3.3935154178$

C,-0.8469300322,3.1766885574,1.1665812761

$\mathrm{H}, 0.08619953,2.9642681707,1.6976673219$

$\mathrm{H},-0.6327691003,3.9092392115,0.3799402298$

$\mathrm{H},-1.5358920876,3.6467949123,1.8776517012$

C,-3.3012734796, 1.9986026067,-0.3590666203

$\mathrm{H},-3.9990454718,2.4388046164,0.3618311407$

$\mathrm{H},-3.1703775705,2.7073196853,-1.1851250623$

$\mathrm{H},-3.7483777435,1.0876475443,-0.7660213152$

C,1.8199690916,2.3839548852,-1.1908527904

$\mathrm{H}, 0.7324503723,2.3433969035,-1.246220955$

$\mathrm{H}, 2.122514005,3.2516723522,-0.5985104867$

$\mathrm{H}, 2.2287940128,2.4773124728,-2.2021068368$

C, $1.9270127493,-2.2129122369,0.5476143766$

$\mathrm{H}, 1.4547942542,-2.2106822249,1.5329495805$

$\mathrm{H}, 1.198717032,-2.5701447461,-0.185147258$

$\mathrm{H}, 2.7919898741,-2.8765250091,0.5567859202$

C,-3.5458359509,-2.1270516328,-2.1110473919

C,-4.2172091212,-1.1667887989,-2.4064955144

$\mathrm{H},-4.8083272345,-0.3203812957,-2.66675823$

C,-2.7341794005,-3.2853712384,-1.7554657133

$\mathrm{H},-3.2596577163,-3.9354396079,-1.0471807516$

$\mathrm{H},-2.4881389848,-3.880403506,-2.6421021938$

$\mathrm{H},-1.7970183978,-2.9540379438,-1.2942081047$

Zero-point correction=

Thermal correction to Energy=

Thermal correction to Enthalpy=

Thermal correction to Gibbs Free Energy=

Sum of electronic and zero-point Energies=

Sum of electronic and thermal Energies=

Sum of electronic and thermal Enthalpies=

Sum of electronic and thermal Free Energies=
0.392277 (Hartree/Particle) 0.421686

0.422630

0.331249

$-3313.455339$

$-3313.425930$

$-3313.424986$

$-3313.516367$ 
Table S24. Cartesian coordinates and energy values for the TS1.

Ge, $0.0212495921,1.7030877565,-0.9516407932$

$\mathrm{Si},-2.1803585552,-0.8700373738,1.0398587993$

$\mathrm{Si},-1.4975770453,1.2803754171,0.890976588$

$\mathrm{N}, 2.4356658213,0.489513172,0.6198511306$

$\mathrm{N}, 1.7544082444,-0.758305998,-0.9964497535$

C,-2.7410268683,-1.7713771238,-1.0919499457

C,-2.6310293045,-3.0033391935,-0.9452794099

C, $1.4959524125,0.4147643602,-0.3584668613$

C,3.2812602821,-0.6199599509, 0.5969363967

$\mathrm{C}, 2.8413663288,-1.4164150889,-0.4224287133$

$\mathrm{C}, 4.4136312576,-0.8019416623,1.5520846641$

C,3.3418141396,-2.7346518408,-0.911614923

$\mathrm{H}, 4.0679016584,-0.8857778917,2.5891248869$

$\mathrm{H}, 5.1233937976,0.0317020578,1.5066447521$

$\mathrm{H}, 4.9628358196,-1.7155287436,1.316116111$

$\mathrm{H}, 4.174852997,-3.0753564571,-0.2936809897$

$\mathrm{H}, 3.6996830444,-2.6807793811,-1.9464440174$

$\mathrm{H}, 2.5628501628,-3.5041414392,-0.8717573018$

C, $-0.848348186,-2.0785623042,1.6709335266$

$\mathrm{H}, 0.1390447006,-1.8412733467,1.2630360157$

$\mathrm{H},-0.7806610719,-2.0252699198,2.7622823132$

$\mathrm{H},-1.0877693251,-3.1134943651,1.4031057959$

C,2.4896569974,1.564418043,1.603904881

$\mathrm{H}, 3.0657606697,2.4143151883,1.2288751976$

$\mathrm{H}, 1.467608296,1.8823718393,1.8139412762$

$\mathrm{H}, 2.9505983753,1.1941243685,2.5200804924$

C, $0.9536013979,-1.2868195216,-2.0980558306$

$\mathrm{H}, 0.1528881492,-1.9308095442,-1.7240946304$

$\mathrm{H}, 0.5072351492,-0.4381903329,-2.6236683317$

$\mathrm{H}, 1.5942332792,-1.8511873957,-2.7775623182$

C,-3.7861817398,-1.2316383129,2.0043799665

$\mathrm{H},-4.1647432717,-2.2287965929,1.7549113242$

$\mathrm{H},-3.610727582,-1.1973261377,3.0845782288$

$\mathrm{H},-4.5704205894,-0.5060815521,1.7674608877$

C, $0.8589005748,3.4983148649,-0.5448893457$

$\mathrm{H}, 1.7966011868,3.582613341,-1.1046938511$

$\mathrm{H}, 0.1718818175,4.2331262765,-0.9767825529$

$\mathrm{H}, 1.0368951131,3.7735461634,0.4975587517$

C,-2.9927331446,2.4687798716,0.5804905611

$\mathrm{H},-3.1056429764,2.6995094379,-0.4843131965$

$\mathrm{H},-3.9374205125,2.0633750891,0.9564049088$

$\mathrm{H},-2.815022999,3.4163217508,1.1024763324$

C,-3.1485728598,-0.6750943487,-2.0095174047

$\mathrm{H},-3.9796510511,-0.1068833,-1.5810794379$

$\mathrm{H},-2.3259194015,0.0297442578,-2.1695238399$

$\mathrm{H},-3.4656282307,-1.0929174542,-2.9713087081$

$\mathrm{H},-2.3979161103,-3.9211717323,-0.4494821022$

Zero-point correction $=$

Thermal correction to Energy=

Thermal correction to Enthalpy=

Thermal correction to Gibbs Free Energy=

Sum of electronic and zero-point Energies=

Sum of electronic and thermal Energies=

Sum of electronic and thermal Enthalpies $=$

Sum of electronic and thermal Free Energies=
0.391703 (Hartree/Particle) 0.420973

0.421917

0.331258

$-3313.399547$

$-3313.370277$

$-3313.369333$

$-3313.459992$ 
Table S25. Cartesian coordinates and energy values for the TS2.

Ge,-0.1084922564,-1.9662266908,0.5910466075

$\mathrm{Si},-2.2034413553,1.1383083015,-0.6104885161$

$\mathrm{Si},-1.554649758,-1.0477757321,-1.1063917924$

$\mathrm{N}, 2.4402810876,-0.6120579195,-0.5907116232$

$\mathrm{N}, 1.7678329938,0.2866453992,1.2464501237$

C,-2.7397130041,1.260963842,1.3526665541

C,-2.5872749227, $2.5118608333,1.4812512367$

C, $1.4663342049,-0.6934663992,0.3524808926$

C,3.3511244928, $0.4021496862,-0.2930306054$

$\mathrm{C}, 2.9176589893,0.9784469877,0.8673578997$

C,4.531012259,0.7156992288,-1.1514430169

C,3.4753307271,2.1158829267,1.6563601818

$\mathrm{H}, 4.2339618493,1.0540387086,-2.1511443496$

$\mathrm{H}, 5.1864818814,-0.1533759718,-1.2777014429$

$\mathrm{H}, 5.124466773,1.5131686492,-0.6999746059$

$\mathrm{H}, 4.3555278851,2.5261893565,1.1575947508$

$\mathrm{H}, 3.7806955803,1.8079356527,2.6632271288$

$\mathrm{H}, 2.7476679443,2.9276929899,1.7663822466$

C,-0.7560244696,2.3150749401,-0.9787211055

$\mathrm{H}, 0.084276454,2.1608093217,-0.294695328$

$\mathrm{H},-0.400725898,2.1070667902,-1.9951721985$

$\mathrm{H},-1.0518807037,3.3662746966,-0.9239869419$

C,2.4681755201,-1.4413391867,-1.7895646607

$\mathrm{H}, 2.9797887504,-2.3892381806,-1.6025901474$

$\mathrm{H}, 1.4374621446,-1.6378707375,-2.0880204766$

$\mathrm{H}, 2.982927365,-0.9072171282,-2.5888347224$

C, $0.962656542,0.5989424277,2.4249499582$

$\mathrm{H}, 0.2391202818,1.3891432012,2.2069508903$

$\mathrm{H}, 0.4197356855,-0.3084774963,2.7050770727$

$\mathrm{H}, 1.6158798866,0.9114468218,3.24160327$

C,-3.7079866539,1.7116729487,-1.6252927504

$\mathrm{H},-4.0414641013,2.7005961248,-1.2933050568$

$\mathrm{H},-3.4498931477,1.7773377638,-2.6870676908$

$\mathrm{H},-4.5506972073,1.0194486042,-1.5290443726$

C, $0.6179324924,-3.6949023472,-0.1563408822$

$\mathrm{H}, 1.5203376792,-3.9698913124,0.3991083507$

$\mathrm{H},-0.1394348085,-4.447049046,0.0851882011$

$\mathrm{H}, 0.8238622333,-3.7510188556,-1.2281468197$

C,-3.0779273927,-2.2474677033,-1.0653792054

$\mathrm{H},-3.17976367,-2.755338964,-0.1004373368$

$\mathrm{H},-4.017879633,-1.7354199459,-1.2954629331$

$\mathrm{H},-2.9385280502,-3.0229045667,-1.8286197873$

C,-3.2325755711,0.0660417377,2.1218706799

$\mathrm{H},-4.0855136867,-0.3878583789,1.606126462$

$\mathrm{H},-2.4511859475,-0.6985641757,2.1879136584$

$\mathrm{H},-3.5449537141,0.3606242232,3.1295384097$

$\mathrm{H},-2.6450767311,3.4981535043,1.8966896032$

Zero-point correction=

Thermal correction to Energy=

Thermal correction to Enthalpy=

Thermal correction to Gibbs Free Energy=

Sum of electronic and zero-point Energies=

Sum of electronic and thermal Energies=

Sum of electronic and thermal Enthalpies=

Sum of electronic and thermal Free Energies $=$
0.390746 (Hartree/Particle)

0.420192

0.421136

0.329882

$-3313.396040$

$-3313.366594$

$-3313.365650$

$-3313.456904$ 
Table S26. Cartesian coordinates and energy values for the TS3.

Ge,-0.0268215167,-1.7851235488,0.2782454539

$\mathrm{Si},-1.9964462537,2.0251354537,-0.42484188$

$\mathrm{Si},-1.913479557,-0.3365091268,-0.2426830718$

$\mathrm{N}, 1.3881022657,-1.5344217785,-2.5111785033$

$\mathrm{N}, 2.233651537,-0.2731568696,-0.9869450644$

C,-2.609818338,0.3229105284,1.4269564224

C,-2.6443690963,1.6763592712,1.3091556839

C, $1.2176915581,-1.1515431507,-1.2171073767$

C, $2.5031004031,-0.9170753625,-3.0848376993$

C,3.0314395846,-0.1105185754,-2.1192123875

C,2.9386540182,-1.1625662948,-4.4911511264

C, $4.2123854333,0.8010765665,-2.1448803704$

$\mathrm{H}, 2.1842491708,-0.843572811,-5.2202245431$

$\mathrm{H}, 3.1473382696,-2.2226951631,-4.6748109638$

$\mathrm{H}, 3.8533440019,-0.6055721149,-4.7038088358$

$\mathrm{H}, 4.6607787691,0.8147543424,-3.1401703515$

$\mathrm{H}, 4.9884099561,0.4875641724,-1.4367704224$

$\mathrm{H}, 3.9341997075,1.8303702109,-1.8918157047$

C,- $0.4279957782,3.1066478478,-0.4749039863$

$\mathrm{H}, 0.2911045583,2.7925812303,0.287606085$

$\mathrm{H}, 0.0630580696,3.0563964034,-1.4535956621$

$\mathrm{H},-0.6737663574,4.1580760719,-0.2834254898$

C, $0.4674055439,-2.4135618106,-3.2184992953$

$\mathrm{H}, 0.7405581701,-3.4646205219,-3.0920543559$

$\mathrm{H},-0.5309388891,-2.2567976652,-2.8136626989$

$\mathrm{H}, 0.4721278042,-2.1677248178,-4.2811892458$

C,2.4470901871, $0.4296152147,0.2726796738$

$\mathrm{H}, 2.1512576521,1.4781067818,0.1842581643$

$\mathrm{H}, 1.8332387194,-0.0602941423,1.0347816272$

$\mathrm{H}, 3.500776161,0.3726476773,0.5566143938$

C, $-3.2403894545,2.8134789848,-1.6318109033$

$\mathrm{H},-3.4771078948,3.8437185364,-1.3398503884$

$\mathrm{H},-2.8346879629,2.843616835,-2.6497970903$

$\mathrm{H},-4.1776768114,2.2497075269,-1.6618245667$

C,-0.644874529,-3.59108397,-0.3954517871

$\mathrm{H}, 0.2271215442,-4.227417198,-0.5808938422$

$\mathrm{H},-1.1618893435,-4.0235954218,0.4675567895$

$\mathrm{H},-1.3208163789,-3.6435245461,-1.2528696605$

C,-3.3226453035,-1.1981625841,-1.2262331954

$\mathrm{H},-3.5604234659,-2.1806347945,-0.8042366017$

$\mathrm{H},-4.235428333,-0.5928746872,-1.2089883017$

$\mathrm{H},-3.041827013,-1.3502532946,-2.2750600119$

C,-2.9531318669,-0.4683899326,2.6589188643

$\mathrm{H},-3.7685139164,-1.176870035,2.4614655797$

$\mathrm{H},-2.0856183222,-1.0709600523,2.9606435018$

$\mathrm{H},-3.249672236,0.1662557626,3.5028840246$

$\mathrm{H},-2.9701654169,2.3305463918,2.1250070804$

Zero-point correction=

Thermal correction to Energy=

Thermal correction to Enthalpy=

Thermal correction to Gibbs Free Energy=

Sum of electronic and zero-point Energies=

Sum of electronic and thermal Energies=

Sum of electronic and thermal Enthalpies=

Sum of electronic and thermal Free Energies $=$
0.395271 (Hartree/Particle)

$$
0.422466
$$

0.423410

0.339067

$-3313.504112$

$-3313.476917$

$-3313.475973$

$-3313.560316$ 
Table S27. Cartesian coordinates and energy values for the TS4.

Ge, $0.1289526685,0.9432458436,-0.6178695699$

Si, $2.4498526128,2.0049505782,0.4452643606$

Si, $1.6449577683,-0.5273037913,0.4380189353$

$\mathrm{N},-1.9978954585,0.175603254,1.4729775268$

$\mathrm{N},-1.4287087151,2.2322761066,1.7096431176$

C,3.2371784623,-0.3430566799,-0.5999716086

C,3.6244332438,0.9551896182,-0.5736268115

C,-1.1712850491,1.1274692472,0.9662211451

C,-2.7495558306, $0.6682824168,2.5382435244$

C,-2.3825614891,1.9738376582,2.694426931

C,-3.7313462671,-0.1674469213,3.2897605311

C,-2.8342877042,3.0022744948,3.6770227984

$\mathrm{H},-3.2510808919,-1.0252409024,3.7744803051$

$\mathrm{H},-4.5196170279,-0.5572757205,2.6357633062$

$\mathrm{H},-4.213146145,0.4244283945,4.0703463431$

$\mathrm{H},-3.5353364939,2.5612162007,4.3881491603$

$\mathrm{H},-3.3439143285,3.8424156578,3.190874572$

$\mathrm{H},-1.9950109311,3.4119090843,4.2498299838$

C, $2.3970745511,3.7626513651,-0.3242200038$

$\mathrm{H}, 2.0736242733,3.7183349618,-1.368330511$

$\mathrm{H}, 1.7199799989,4.440604816,0.2077847546$

$\mathrm{H}, 3.3962728864,4.2179011483,-0.2849644006$

C,-2.1212710521,-1.1815782586,0.954797237

$\mathrm{H},-3.0682850405,-1.3019647777,0.4207858671$

$\mathrm{H},-1.2922548485,-1.3662828167,0.2737415921$

$\mathrm{H},-2.0768386567,-1.8995794115,1.7763669755$

C,- $0.8137492072,3.5389755913,1.5004300072$

$\mathrm{H}, 0.0219663416,3.6936317637,2.1876901957$

$\mathrm{H},-0.4454487442,3.5847157948,0.4764961436$

$\mathrm{H},-1.5598143166,4.3203768694,1.6575155914$

C,3.0375671627,2.3349808078,2.2398604548

$\mathrm{H}, 3.7806631864,3.1416812189,2.2842620835$

$\mathrm{H}, 2.203386707,2.6183774196,2.8933883714$

$\mathrm{H}, 3.4738216101,1.422110258,2.6549360893$

C,-1.2561315445,0.6191253498,-2.0651817377

$\mathrm{H},-1.8991157392,1.4971026304,-2.187284061$

$\mathrm{H},-0.6963753706,0.4835360377,-2.9952361878$

$\mathrm{H},-1.8826746648,-0.2663402946,-1.9105441193$

C, $1.4452870669,-2.3895701224,0.9231978458$

$\mathrm{H}, 1.296995774,-3.0263459749,0.0419898906$

$\mathrm{H}, 2.3552805682,-2.732593515,1.4297791335$

$\mathrm{H}, 0.6083744155,-2.5595642651,1.60827085$

C,3.9485644468,-1.4289441335,-1.3668764446

$\mathrm{H}, 4.2246781869,-2.2630694957,-0.7090882209$

$\mathrm{H}, 3.3001867216,-1.8536008519,-2.1445842735$

$\mathrm{H}, 4.8631547741,-1.0621554736,-1.848703962$

$\mathrm{H}, 4.4279926697,1.3271522293,-1.2191888116$

Zero-point correction $=$

Thermal correction to Energy=

Thermal correction to Enthalpy=

Thermal correction to Gibbs Free Energy=

Sum of electronic and zero-point Energies=

Sum of electronic and thermal Energies=

Sum of electronic and thermal Enthalpies=

Sum of electronic and thermal Free Energies=
0.393820 (Hartree/Particle)

0.421421

0.422365

0.337256

$-3313.458895$

$-3313.431294$

$-3313.430350$

$-3313.515458$ 
Table S28. Cartesian coordinates and energy values for the TS5.

Ge,-0.7114271033,-2.0052977511,-0.3254021073

$\mathrm{Si},-1.3189207124,-0.9570953909,1.7993142133$

$\mathrm{Si},-1.2120122069,0.060321753,-1.1640384587$

$\mathrm{N}, 1.7758149526,1.4550277416,0.2336347557$

$\mathrm{N}, 2.1691202746,-0.0322530641,-1.2423542814$

C, $-1.9240067032,0.7972427176,1.4109523831$

$\mathrm{H},-2.3477808204,1.4342079527,2.1978622207$

C,- $-1.9042818116,1.2647416781,0.1447657595$

C, $1.2370958294,0.9076484718,-0.8955143471$

$\mathrm{C}, 2.9837121271,0.852752212,0.6054856423$

C,3.2273230661,-0.1052054057,-0.3347842841

$\mathrm{C}, 3.7593510535,1.2602554778,1.8139481016$

C,4.3555002805,-1.0721773402,-0.4742634582

$\mathrm{H}, 3.1769894439,1.1304510064,2.7336861996$

$\mathrm{H}, 4.072035229,2.3104482917,1.7681138251$

$\mathrm{H}, 4.6627602151,0.6535342162,1.9081112264$

$\mathrm{H}, 5.0665897883,-0.9487273889,0.3455680409$

$\mathrm{H}, 4.9061518131,-0.9299447894,-1.4119796022$

$\mathrm{H}, 4.0042004114,-2.1104967543,-0.4551931156$

C,-2.4228186446,2.6306592689,-0.2441825351

$\mathrm{H},-2.7362717212,3.2184977413,0.6263856686$

$\mathrm{H},-1.6699939335,3.2080799688,-0.7966755821$

$\mathrm{H},-3.2846691344,2.5460250818,-0.9194365386$

C,-2.6425903047,-1.833727986,2.8515328107

$\mathrm{H},-3.5976426211,-1.9013755119,2.3208594329$

$\mathrm{H},-2.3294728662,-2.8507714305,3.1098746276$

$\mathrm{H},-2.8190488722,-1.2888868072,3.7870805751$

C,1.1834026743,2.5688754208,0.9566906996

$\mathrm{H}, 1.9160221777,3.3728337506,1.0780130903$

$\mathrm{H}, 0.3402719695,2.9384784717,0.3792411016$

$\mathrm{H}, 0.8251769216,2.2567826247,1.9414895664$

C,2.0601103299,-0.8760611274,-2.4233781559

$\mathrm{H}, 1.8158849143,-1.9074909165,-2.1519761762$

$\mathrm{H}, 1.2626726767,-0.4829033446,-3.0503881608$

$\mathrm{H}, 2.9967591979,-0.8561371288,-2.9880831937$

C, $0.2941058538,-0.9060459643,2.8238738975$

$\mathrm{H}, 0.2373129812,-0.1764255149,3.6401272435$

$\mathrm{H}, 0.4924053494,-1.8873076265,3.2693878962$

$\mathrm{H}, 1.1492133594,-0.6536158858,2.1879736727$

C,-2.8609813196,-1.2983839256,-1.318292018

$\mathrm{H},-3.0467703885,-2.2229185719,-0.7526905812$

$\mathrm{H},-3.6631516281,-0.6140675483,-1.0271947388$

$\mathrm{H},-2.9340431956,-1.5656981125,-2.3740375116$

C,-1.3391547185,0.7403349093,-2.9333602161

$\mathrm{H},-2.3606414927,1.0718016239,-3.1496744914$

$\mathrm{H},-0.6597252973,1.585663305,-3.062756222$

$\mathrm{H},-1.0825323942,-0.0252543983,-3.673428874$

Zero-point correction $=$

Thermal correction to Energy=

Thermal correction to Enthalpy=

Thermal correction to Gibbs Free Energy=

Sum of electronic and zero-point Energies=

Sum of electronic and thermal Energies=

Sum of electronic and thermal Enthalpies=

Sum of electronic and thermal Free Energies=
0.394019 (Hartree/Particle)

0.421601

0.422546

0.337135

$-3313.466887$

$-3313.439304$

$-3313.438360$

$-3313.523771$ 
Table S29. Cartesian coordinates and energy values for the TS6.

$\mathrm{Ge},-1.72356729,-1.6704411793,-0.5166243579$

$\mathrm{Si},-0.0252167921,0.1407105444,-0.5781365928$

$\mathrm{Si}, 2.1269924385,-0.4948237724,0.1452080877$

C,3.6830765297, $0.1896391889,-0.6996714705$

$\mathrm{H}, 3.9155313228,-0.3762740954,-1.6085303849$

$\mathrm{H}, 3.557435875,1.2398851023,-0.9797260934$

$\mathrm{H}, 4.5527495797,0.1207520212,-0.0357115762$

C, $2.4386984393,-2.3055855197,0.6242592587$

$\mathrm{H}, 3.2526068525,-2.3912194941,1.3535266358$

$\mathrm{H}, 1.5447868506,-2.7623760498,1.0610393416$

$\mathrm{H}, 2.7171904439,-2.8981723521,-0.2542481818$

C,- $-0.2670511985,1.3081794201,-2.07706305$

$\mathrm{H}, 0.5297952164,2.0579165443,-2.1157439503$

$\mathrm{H},-0.2469904931,0.7469477933,-3.0172752111$

$\mathrm{H},-1.2258086466,1.8349459226,-2.0231462712$

C,-3.3045958984,-0.6238435654,0.1204126626

$\mathrm{H},-3.7584337387,-0.2661944798,-0.8190544718$

$\mathrm{H},-4.044436924,-1.2728565324,0.5966619113$

$\mathrm{H},-3.1130334186,0.2494561807,0.7469626761$

C, $0.2201366633,1.0206206997,1.1148874385$

C, $1.4430648978,0.5941011376,1.5320179392$

$\mathrm{H}, 1.8604310593,0.8951061555,2.4987033539$

C, $-0.6903993293,1.9362337453,1.8886628011$

$\mathrm{H},-0.2215617903,2.2982405777,2.8111426739$

$\mathrm{H},-0.9820254837,2.8074168158,1.2890538715$

$\mathrm{H},-1.6214563955,1.4238384611,2.16267694$

Zero-point correction=

Thermal correction to Energy=

Thermal correction to Enthalpy=

Thermal correction to Gibbs Free Energy=

Sum of electronic and zero-point Energies=

Sum of electronic and thermal Energies=

Sum of electronic and thermal Enthalpies=

Sum of electronic and thermal Free Energies $=$
0.208432 (Hartree/Particle)

0.224881

0.225826

0.164066

$-2930.159139$

$-2930.142689$

$-2930.141745$

$-2930.203504$

Table S30. Cartesian coordinates and energy values for the TS7.

Ge,-1.4324142702,-0.7627807001,0.504979466

Si, $0.8383201406,-1.2872220021,0.2101322005$

$\mathrm{Si},-0.2670084231,1.4326312989,0.0739172508$

C, $0.1101381416,2.3571633714,1.6965711966$

$\mathrm{H},-0.8169846212,2.5633151108,2.2407845451$

$\mathrm{H}, 0.7581697821,1.7717888012,2.3566302121$

$\mathrm{H}, 0.605643366,3.3146169806,1.4981480393$

C, $-1.3412965303,2.5629049133,-1.0099283997$

$\mathrm{H},-0.8296574761,3.5157424973,-1.1935778999$

$\mathrm{H},-1.5622849326,2.1039713322,-1.9776575613$

$\mathrm{H},-2.2938034897,2.7874440905,-0.5185107222$

C, $1.9658111635,-2.3625993928,1.2895625373$

$\mathrm{H}, 2.6956006001,-1.7567198488,1.8399925141$

$\mathrm{H}, 1.3965790459,-2.9669536582,1.9992141699$

$\mathrm{H}, 2.5402837381,-3.0396846577,0.6425726335$

C,-2.035718154,-0.937139379,-1.4437664124

$\mathrm{H},-2.3140139984,-1.9854559321,-1.5762425425$

$\mathrm{H},-2.9462799679,-0.338222603,-1.5252623284$

$\mathrm{H},-1.3365201341,-0.6427498437,-2.2279392241$

C, $1.9336358215,-0.1387452901,-0.794353104$ 
C, $1.3929398243,1.1077836255,-0.8123013053$

$\mathrm{H}, 1.9629308027,1.917700792,-1.2856522453$

C,3.2810657428,-0.4951293545,-1.3751307135

$\mathrm{H}, 3.7588784218,0.367357878,-1.8519963539$

$\mathrm{H}, 3.9592736199,-0.8718789887,-0.5996006203$

H,3.1958858168,-1.289394561,-2.1269909226

Zero-point correction=

Thermal correction to Energy=

Thermal correction to Enthalpy=

Thermal correction to Gibbs Free Energy=

Sum of electronic and zero-point Energies=

Sum of electronic and thermal Energies=

Sum of electronic and thermal Enthalpies=

Sum of electronic and thermal Free Energies=
0.209134 (Hartree/Particle)

0.225301

0.226246

0.166087

$-2930.170308$

$-2930.154141$

$-2930.153196$

$-2930.213355$

Table S31. Cartesian coordinates and energy values for the TS8.

Ge,-0.619337277,1.0795408037,-0.4108733653

Si, $1.4700535897,0.1538155396,-0.5567424223$

$\mathrm{Si},-1.1990604878,-1.309043731,-0.3270613691$

C,-2.3686343964,-1.7669590868,-1.7537147129

$\mathrm{H},-3.3454056964,-1.2908032682,-1.6161239951$

$\mathrm{H},-1.9694323371,-1.4278466717,-2.7151256632$

$\mathrm{H},-2.5278656508,-2.8499354985,-1.8132361203$

C,-2.0136760379,-1.8295310741,1.3144526781

$\mathrm{H},-2.2572062761,-2.8983188067,1.3082522906$

$\mathrm{H},-1.3590337091,-1.6390907499,2.1704507965$

$\mathrm{H},-2.9454029683,-1.2763014117,1.4735163538$

C,3.167537111, $0.9342610585,-0.2467117408$

$\mathrm{H}, 3.8190399997,0.7169569906,-1.1027940432$

$\mathrm{H}, 3.1030886229,2.0193357744,-0.1372808904$

$\mathrm{H}, 3.6537530526,0.5154663543,0.6414425586$

C,0.3000126651,1.2095167914,1.5436963699

$\mathrm{H}, 0.6486235778,2.2375536505,1.6439559741$

$\mathrm{H},-0.6206905743,1.0868168251,2.1194938802$

$\mathrm{H}, 1.0393641072,0.5317763637,1.9856266117$

C,1.6157615813,-1.7235339351,-0.5789256847

C, $0.4046315462,-2.3293449987,-0.5159737946$

$\mathrm{H}, 0.3722988999,-3.4257375874,-0.5478413522$

C,2.9318801704,-2.4629918968,-0.671565006

$\mathrm{H}, 2.7849833008,-3.5475789932,-0.7107005371$

$\mathrm{H}, 3.4997836258,-2.1685429672,-1.5628434803$

$H, 3.5732196507,-2.2413885249,0.1904550243$

Zero-point correction=

Thermal correction to Energy=

Thermal correction to Enthalpy=

Thermal correction to Gibbs Free Energy=

Sum of electronic and zero-point Energies=

Sum of electronic and thermal Energies=

Sum of electronic and thermal Enthalpies=

Sum of electronic and thermal Free Energies=
0.209089 (Hartree/Particle) 0.225161

0.226105

0.166503 $-2930.173880$ $-2930.157808$ $-2930.156864$ $-2930.216466$ 
Table S32. Cartesian coordinates and energy values for the TS9.

Ge, $0.8019194354,-0.9799663704,-2.1572447618$

Si, $1.9472673355,-0.4078110891,1.3239894738$

$\mathrm{Si}, 1.5148883178,-2.1004860715,-0.0990318175$

$\mathrm{N},-2.1914202255,-1.2291801176,-1.2802984952$

$\mathrm{N},-1.529169283,0.8126652019,-1.4172741058$

$\mathrm{C}, 3.2850122874,2.3650677461,0.5268120207$

C, $2.8192980472,1.4830594508,-0.185604019$

C, $-1.101099717,-0.4703117336,-1.5633456981$

C,-3.2954372156,-0.4363175823,-0.9659457062

C,-2.8718955613,0.8598570952,-1.0445862554

C,-4.6339157613,-1.0002383351,-0.6216487613

C,-3.5992451704,2.1409509538,-0.8057163437

$\mathrm{H},-4.604553391,-1.5981328734,0.2967225227$

$\mathrm{H},-5.0237947055,-1.6407775941,-1.4205914966$

$\mathrm{H},-5.3533571286,-0.1940903209,-0.4646998877$

$\mathrm{H},-4.6245782893,1.9406161122,-0.4888579684$

$\mathrm{H},-3.647809131,2.760683527,-1.70874448389$

$\mathrm{H},-3.1209440015,2.7390980515,-0.0219420828$

C, $0.4624727117,0.5377722243,2.0573694235$

$\mathrm{H},-0.3652185097,0.573504805,1.3425338591$

$\mathrm{H}, 0.0980787223,0.0542718519,2.9703025166$

$\mathrm{H}, 0.742424689,1.5662946072,2.3106411893$

C,-2.1816445411,-2.6882530482,-1.2665899348

$\mathrm{H},-2.3875705229,-3.0898893915,-2.2621139978$

$\mathrm{H},-1.1950775637,-3.0164464445,-0.9328683342$

$\mathrm{H},-2.9386868027,-3.0477124232,-0.568983058$

C, $-0.6853319557,1.99099829,-1.5908322382$

$\mathrm{H},-0.3700087468,2.3888149193,-0.6225935165$

$\mathrm{H}, 0.1984963465,1.6839413247,-2.1560700695$

$\mathrm{H},-1.232115828,2.759623816,-2.1413404086$

C,3.2675295607,-0.5336341343,2.6923482133

$\mathrm{H}, 3.5886253204,0.4619070641,3.018786805$

$\mathrm{H}, 2.8673310193,-1.0542383228,3.5694373971$

$\mathrm{H}, 4.1506431898,-1.0781199606,2.3467740591$

C, $0.3708028284,-2.474622448,-3.4465081134$

$\mathrm{H},-0.2847131148,-2.0891917426,-4.234818497$

$\mathrm{H}, 1.322156323,-2.7272617409,-3.9253255853$

$\mathrm{H},-0.0580845702,-3.3941347168,-3.0398861797$

C, $3.2138767741,-2.8936214752,-0.6101195076$

$\mathrm{H}, 3.0440176093,-3.9048158104,-0.9967620275$

$\mathrm{H}, 3.7081001801,-2.3165718817,-1.3992042804$

H,3.9002056242,-2.9807946942,0.2384628609

C,3.8284393394,3.3201486776,1.483522126

$\mathrm{H}, 4.2818968683,2.816249398,2.3461394471$

$\mathrm{H}, 4.6073687614,3.9391903497,1.0213992382$

$\mathrm{H}, 3.0509781934,3.9943922974,1.860002612$

$\mathrm{H}, 2.5151775119,1.0116772894,-1.1133849074$

Zero-point correction $=$

Thermal correction to Energy=

Thermal correction to Enthalpy=

Thermal correction to Gibbs Free Energy=

Sum of electronic and zero-point Energies=

Sum of electronic and thermal Energies=

Sum of electronic and thermal Enthalpies=

Sum of electronic and thermal Free Energies=
0.390916 (Hartree/Particle)

0.420658

0.421603

0.328325

$-3313.405515$

$-3313.375773$

$-3313.374829$

$-3313.468106$ 
Table S33. Cartesian coordinates and energy values for the TS10.

Ge, $0.7803909491,-1.3986348432,-2.1001629491$

Si,2.6979067262,-0.2436155945,1.0808562828

Si, $1.5836961558,-1.9078315268,-0.0389411445$

$\mathrm{N},-2.1427014296,-1.0393485367,-1.3155780551$

$\mathrm{N},-1.2688994633,0.8697109419,-1.817165376$

C,3.3053835149,1.9261163496,-1.0075246411

C,3.0320580785, $1.0800608937,-0.1725039688$

C, $-1.0334724375,-0.4691980265,-1.8562573705$

C,-3.048621597,-0.0662627503,-0.8916459297

C,-2.4982549285, $1.1419711226,-1.2136856892$

C,- $-4.3495615352,-0.3941582548,-0.2378132687$

C,-3.026905552,2.5248031604,-1.022317434

$\mathrm{H},-4.2104773385,-0.8960313614,0.7265463899$

$\mathrm{H},-4.9695077454,-1.0451848578,-0.8653654188$

$\mathrm{H},-4.9180285852,0.5190706743,-0.0519777448$

$\mathrm{H},-4.0126643487,2.4879856704,-0.5547228191$

$\mathrm{H},-3.1348274439,3.0592042797,-1.973459359$

$\mathrm{H},-2.3788994649,3.1256088273,-0.3741586937$

C, $1.7455726692,0.5797926197,2.5109553257$

$\mathrm{H}, 0.7983940101,0.9977008648,2.1565218027$

$\mathrm{H}, 1.5092069619,-0.1629887274,3.281780714$

$\mathrm{H}, 2.3269090897,1.3831225692,2.9800158778$

C,-2.2204324702,-2.4589683779,-1.0109841347

$\mathrm{H},-2.0423358935,-3.0468033169,-1.9120824577$

$\mathrm{H},-1.4489419756,-2.7000091783,-0.2645638581$

$\mathrm{H},-3.2081854939,-2.699489643,-0.6208043178$

C, $-0.3061167366,1.8710266723,-2.267932812$

$\mathrm{H}, 0.6092478286,1.8022936815,-1.6729834991$

$\mathrm{H},-0.0600378198,1.7090269218,-3.3188693893$

$\mathrm{H},-0.7411847367,2.8630073747,-2.154331337$

C, $4.3662904986,-0.8047844415,1.8066541469$

$\mathrm{H}, 4.8551607688,0.0076938859,2.3564351741$

$\mathrm{H}, 4.2174673608,-1.6389792575,2.5020170896$

$\mathrm{H}, 5.0398814432,-1.1452324051,1.0156593327$

C, $0.3939609642,-2.8671305378,-3.3493754083$

$\mathrm{H},-0.3438808746,-2.5706712341,-4.1002410523$

$\mathrm{H}, 1.3289651129,-3.1134378332,-3.8579906225$

$\mathrm{H}, 0.051025579,-3.7615129326,-2.8244577579$

C, $1.4775519076,-3.7276299178,0.5868748051$

$\mathrm{H}, 0.9108705494,-4.3251384949,-0.1360256329$

$\mathrm{H}, 2.4690881634,-4.1820705869,0.673378145$

$\mathrm{H}, 0.9745654533,-3.8155077181,1.5543202601$

C,3.6593580708,2.9246065438,-2.011741166

$\mathrm{H}, 4.7268958213,3.1687519747,-1.9646318599$

$\mathrm{H}, 3.4508476462,2.5598189825,-3.0244702634$

$\mathrm{H}, 3.1029508625,3.8589741306,-1.8701858741$

$\mathrm{H}, 1.4684947743,-0.2842395563,-2.9063732013$

Zero-point correction=

Thermal correction to Energy=

Thermal correction to Enthalpy=

Thermal correction to Gibbs Free Energy=

Sum of electronic and zero-point Energies=

Sum of electronic and thermal Energies=

Sum of electronic and thermal Enthalpies=

Sum of electronic and thermal Free Energies=
0.391531 (Hartree/Particle)

0.420199

0.421144

0.330492

$-3313.445747$

$-3313.417079$

$-3313.416134$

$-3313.506786$ 
Table S34. Cartesian coordinates and energy values for the TS11.

Ge, $0.3086985836,-0.2799780806,-1.5073020295$

$\mathrm{Si}, 2.9371419602,1.7009053298,0.2869667178$

$\mathrm{Si}, 1.1448351279,0.2331483963,0.7203730638$

$\mathrm{N}, 0.7504582538,2.02358954,-3.3554228353$

$\mathrm{N}, 2.1982032576,0.4787942511,-3.7652473084$

$\mathrm{C}, 2.9362238506,-0.8425616695,0.052433351$

C,3.6209654196, $0.1419515859,-0.4725948452$

C, $1.1344451059,0.7790444278,-2.9905414787$

C, $1.5681980423,2.5148612655,-4.3705370237$

C,2.4919086947, 1.5370301281,-4.6221885209

C, $1.3881886129,3.866525813,-4.9766415589$

C,3.6347292462,1.5126532591,-5.5818266408

$\mathrm{H}, 1.5503806203,4.6662791386,-4.2448604733$

$\mathrm{H}, 0.3852200106,3.9957210059,-5.3984024037$

$\mathrm{H}, 2.1067253997,4.0136341598,-5.7848270126$

$\mathrm{H}, 3.677725345,2.4506276637,-6.1384529353$

$\mathrm{H}, 3.5414614068,0.6996181589,-6.3103709008$

$\mathrm{H}, 4.5910957901,1.3897366002,-5.0627714449$

C,2.929853242,3.2963466679,-0.7712050638

$\mathrm{H}, 2.5643465404,3.1245833232,-1.7872624208$

$\mathrm{H}, 2.3054696141,4.0703929502,-0.3088659792$

$\mathrm{H}, 3.9460545548,3.7007222674,-0.8489953631$

C,- $0.331444172,2.7575030937,-2.7055841588$

$\mathrm{H},-1.2983360162,2.5066693235,-3.148134003$

$\mathrm{H},-0.3346686846,2.4993611964,-1.6450096229$

$\mathrm{H},-0.1529887676,3.8276903002,-2.8038729608$

C,3.0147089804,-0.7354330279,-3.6253610661

$\mathrm{H}, 3.6625536165,-0.6225139187,-2.7463420252$

$\mathrm{H}, 2.3530048074,-1.5890173225,-3.4803518662$

$\mathrm{H}, 3.5942503939,-0.8772258991,-4.5367145215$

C,3.8991475061,2.1771590406,1.8570540867

$\mathrm{H}, 4.932877083,2.4457249935,1.6077492133$

$\mathrm{H}, 3.4412252461,3.0356822093,2.3623437259$

$\mathrm{H}, 3.9275854032,1.3424981437,2.5629586856$

C,-1.6203263308,-0.3067422174,-1.9438785356

$\mathrm{H},-1.7947789596,-0.4040016487,-3.0187882255$

$\mathrm{H},-2.02662714,-1.1990111311,-1.4590331642$

$\mathrm{H},-2.1566905603,0.5618264637,-1.5552641915$

C,- $0.5830102835,0.5294454756,1.6104335977$

$\mathrm{H},-1.207534472,-0.3720044522,1.5730756267$

$\mathrm{H},-0.4203996503,0.7750552444,2.666007281$

$\mathrm{H},-1.1579142129,1.3479681194,1.1573418278$

C,3.0231083735,-2.3281886454,0.1808796386

$\mathrm{H}, 3.0931169175,-2.6239283048,1.233729762$

$\mathrm{H}, 2.1272598065,-2.8102976223,-0.232058256$

$\mathrm{H}, 3.9086456663,-2.7105073622,-0.3427720027$

$\mathrm{H}, 0.7412120306,-1.6917421442,-1.9767173891$

Zero-point correction=

Thermal correction to Energy=

Thermal correction to Enthalpy=

Thermal correction to Gibbs Free Energy=

Sum of electronic and zero-point Energies=

Sum of electronic and thermal Energies=

Sum of electronic and thermal Enthalpies=

Sum of electronic and thermal Free Energies $=$
0.390891 (Hartree/Particle) 0.418783

0.419727

0.334042 $-3313.421672$

$-3313.393779$

$-3313.392835$

$-3313.478520$ 
Table S35. Cartesian coordinates and energy values for the TS12.

Ge, $0.2446559933,-1.353806893,-0.5537066195$

Si,2.151798598,1.8923791525,0.3566453507

Si,2.1137112944,-0.4343851089,0.7379968155

$\mathrm{N},-2.269423924,-0.5178256224,0.8176723334$

$\mathrm{N},-2.2065127449,0.3970706485,-1.133745487$

C,3.2246262299, $-0.095152913,-0.8191861705$

$\mathrm{C}, 3.0200846953,1.2080538251,-1.1315346164$

C, $-1.546402314,-0.4729964068,-0.3283131724$

C,-3.3904113722,0.3065244127, 0.7356775882

C,-3.345753724,0.8916034176, -0.4981334511

C,- $-4.3760886541,0.4530833477,1.8464860199$

C, $-4.2584589533,1.8803338532,-1.1426254677$

$\mathrm{H},-3.9211265864,0.8901484725,2.7428116647$

$\mathrm{H},-4.8167597773,-0.5088138989,2.1309937061$

$\mathrm{H},-5.1911158485,1.1110769578,1.5400536393$

$\mathrm{H},-5.0858456981,2.1227813535,-0.4734470327$

$\mathrm{H},-4.6874692084,1.4943828187,-2.0742028414$

$\mathrm{H},-3.7386381111,2.8152510647,-1.3797407696$

C, $0.5303971496,2.8747304527,0.1049377357$

$\mathrm{H},-0.1845914193,2.2926232285,-0.4848418447$

$\mathrm{H}, 0.0531405937,3.1339810106,1.0572654586$

$\mathrm{H}, 0.7295157824,3.8090679764,-0.4330590745$

C, $-1.9124447196,-1.343322535,1.9671425348$

$\mathrm{H},-2.2794987579,-2.3656921693,1.8440080804$

$\mathrm{H},-0.8259447474,-1.359417083,2.0662072357$

$\mathrm{H},-2.3407545045,-0.9126521441,2.871619469$

C, $-1.7913574156,0.8098474679,-2.4737938229$

$\mathrm{H},-1.5669894679,1.8790187472,-2.4818524142$

$\mathrm{H},-0.8947919528,0.2611395138,-2.7523027335$

$\mathrm{H},-2.5877205442,0.5973979895,-3.1914275838$

C,3.2307687987,2.9402737916,1.5175988039

$\mathrm{H}, 3.5108242649,3.8760064829,1.0181468791$

$\mathrm{H}, 2.7124696066,3.1969255349,2.4484929258$

$\mathrm{H}, 4.1539398329,2.4117966613,1.772798563$

C,- $0.1532810774,-3.2840612227,-0.4160885124$

$\mathrm{H},-1.0675273966,-3.5581239091,-0.9470643495$

$\mathrm{H}, 0.6845171362,-3.7903792494,-0.903158985$

$\mathrm{H},-0.2060342739,-3.6228782893,0.6207367723$

C,2.170023199,-1.7067776993,2.2256084444

$\mathrm{H}, 2.0639709067,-2.7489232243,1.8998433922$

$\mathrm{H}, 3.1330320784,-1.6122826972,2.7399654548$

$\mathrm{H}, 1.3898599487,-1.4967948392,2.9704624376$

C,3.9666404604,-1.1378781505,-1.6219524289

$\mathrm{H}, 4.7109879335,-1.676757828,-1.0201363626$

$\mathrm{H}, 3.2653205263,-1.8949564244,-2.0099837945$

$\mathrm{H}, 4.4730808938,-0.6709508683,-2.4740153704$

$\mathrm{H}, 0.4392312706,-1.1350990059,-2.0693704$

Zero-point correction=

Thermal correction to Energy=

Thermal correction to Enthalpy=

Thermal correction to Gibbs Free Energy=

Sum of electronic and zero-point Energies=

Sum of electronic and thermal Energies=

Sum of electronic and thermal Enthalpies=

Sum of electronic and thermal Free Energies=
0.390046 (Hartree/Particle)

0.418123

0.419068

0.332153

$-3313.414642$

$-3313.386564$

$-3313.385620$

$-3313.472535$ 
Table S36. Cartesian coordinates and energy values for the TS13.

$\mathrm{Ge}, 2.0528116813,-0.7299908579,-2.0622633858$

Si,2.6139317812,1.5252832805,0.1727571338

Si, $1.4845082974,-0.5014105017,0.331643614$

$\mathrm{N},-0.4738929311,-0.3367360511,-3.9105518636$

$\mathrm{N}, 1.3364659902,0.2271110937,-4.9189693626$

C,3.327630507,-0.9504866319,0.387526267

$\mathrm{C}, 3.8526618703,0.1622239531,-0.2535270219$

C, $0.8747153061,-0.3474596009,-3.7788519715$

C, $-0.8642020999,0.2265985686,-5.1275036859$

C, $0.2872758809,0.5825204554,-5.7696207698$

C,-2.2942494243,0.3614030984,-5.5318353175

C, $0.5027388197,1.2292289785,-7.0971086743$

$\mathrm{H},-2.8527327541,1.0107786411,-4.8476843425$

$\mathrm{H},-2.8046131407,-0.6083044326,-5.5553202615$

$\mathrm{H},-2.3666190659,0.7965446609,-6.5304170723$

$\mathrm{H},-0.4516182658,1.368884969,-7.6085905876$

$\mathrm{H}, 1.1416318302,0.6212251504,-7.7473697248$

$\mathrm{H}, 0.972006237,2.2152969597,-7.0012300362$

C,2.1734538452,2.8267759818,-1.1506486853

$\mathrm{H}, 1.8173480495,2.3351925972,-2.0609659813$

$\mathrm{H}, 1.398217177,3.5162241773,-0.798962663$

$\mathrm{H}, 3.0547666388,3.4240787256,-1.412425806$

C,-1.3969790465,-0.8811795488,-2.9219422851

$\mathrm{H},-1.8973738469,-1.7726119269,-3.3115406947$

$\mathrm{H},-0.8324006598,-1.1475884833,-2.0291754828$

$\mathrm{H},-2.1498514849,-0.1352583055,-2.6553749383$

C,2.7540808798,0.4152713272,-5.2193133615

$\mathrm{H}, 2.8925216653,1.3466643171,-5.7704120757$

H,3.3056094461, $0.4714901676,-4.2784855219$

$\mathrm{H}, 3.1400031862,-0.416380913,-5.8158743547$

C,3.0590293805,2.4326216339,1.7774621729

$\mathrm{H}, 3.9505713273,3.053705012,1.6303204929$

$\mathrm{H}, 2.2452066476,3.0854105965,2.1118920938$

$\mathrm{H}, 3.2769538156,1.7159350139,2.573834629$

C,2.7369350644,-2.5169837687,-2.6069805326

$\mathrm{H}, 2.6269071154,-2.6694164236,-3.6834053989$

$\mathrm{H}, 3.7987590412,-2.517017007,-2.3566837444$

$\mathrm{H}, 2.2300449133,-3.3288697058,-2.0822295443$

C,- $0.0792487187,-1.3767451242,1.0617094083$

$\mathrm{H},-0.0843846002,-2.4524349994,0.8486736432$

$\mathrm{H},-0.0986960752,-1.2472233117,2.1503805857$

$\mathrm{H},-1.001928112,-0.9385963497,0.6605214521$

C,4.0176482792,-2.2822612721,0.5540991005

$\mathrm{H}, 4.2702523369,-2.4660456068,1.6080823877$

$\mathrm{H}, 3.4018222585,-3.1335921784,0.2340674511$

$\mathrm{H}, 4.9581649519,-2.2983426715,-0.0102188924$

$\mathrm{H}, 3.551833205,0.0314592431,-1.8653423612$
Zero-point correction $=$
Thermal correction to Energy=
Thermal correction to Enthalpy=
0.387984 (Hartree/Particle)
0.416366
0.417310
Thermal correction to Gibbs Free Energy $=\quad 0.328563$
Sum of electronic and zero-point Energies=
$-3313.392404$
Sum of electronic and thermal Energies=
Sum of electronic and thermal Enthalpies=
$-3313.364022$
$-3313.363078$
Sum of electronic and thermal Free Energies=
$-3313.451825$ 
Table S37. Cartesian coordinates and energy values for the TS14.

$\mathrm{Ge}, 0.2715162656,0.6314326278,1.0127020985$

$\mathrm{Si}, 2.4938984379,-0.6082954388,-1.4814021991$

$\mathrm{Si}, 1.3409673263,1.2835800136,-1.0992995413$

$\mathrm{N},-2.5566352627,0.8382586682,-0.3462820805$

$\mathrm{N},-2.1645058029,-1.1090440101,0.4727553688$

C,3.2832274901,-1.2487625539,1.7108438285

C,3.6227129149,-1.5541214419,0.5712648586

C, $-1.6090638767,0.1225848972,0.3117837219$

C,-3.6971236882,0.073566647,-0.5914102999

C,-3.4449561715,-1.1654061947,-0.0756249828

C,-4.9018005529,0.6056764879,-1.2939557385

C,-4.281330495,-2.400948804,-0.0454791671

$\mathrm{H},-4.6819478939,0.8885263373,-2.3300176008$

$\mathrm{H},-5.3103927911,1.4888849081,-0.7902723686$

$\mathrm{H},-5.6878399038,-0.1517439381,-1.3211044279$

$\mathrm{H},-5.2396601268,-2.22221973,-0.5369616796$

$\mathrm{H},-4.4910030906,-2.7292456775,0.9792097876$

$\mathrm{H},-3.7940348149,-3.2349051638,-0.5635853301$

C, $1.3901838184,-2.1557171372,-1.6398967179$

$\mathrm{H}, 0.6024112031,-2.0735613315,-0.8850114817$

$\mathrm{H}, 0.9214581757,-2.2294956778,-2.6266849237$

$\mathrm{H}, 1.9465635466,-3.081072161,-1.4560184292$

C,-2.384219654,2.2276509154,-0.7542123854

$\mathrm{H},-2.7129079098,2.9096349827,0.0344640844$

$\mathrm{H},-1.3244535678,2.3861028885,-0.962743658$

$\mathrm{H},-2.963141886,2.4156898368,-1.6592022807$

C,-1.518023859,-2.2355126368,1.1394900018

$\mathrm{H},-1.3174806141,-3.038995905,0.4253737957$

$\mathrm{H},-0.5744726288,-1.873560845,1.5572443595$

$\mathrm{H},-2.1594585943,-2.6147658552,1.939877501$

C,3.951933472,-0.7694359159,-2.7051365941

$\mathrm{H}, 4.5859117747,-1.6348821911,-2.4763853781$

$\mathrm{H}, 3.580757773,-0.9016093998,-3.7274448064$

$\mathrm{H}, 4.5829041425,0.1240344434,-2.6879276796$

C, $-0.2083473422,2.4036641714,1.8734275423$

$\mathrm{H},-1.0084484102,2.2482427255,2.6045636773$

$\mathrm{H}, 0.6859084836,2.6912700577,2.4357130676$

$\mathrm{H},-0.4777802787,3.2339419977,1.2150526356$

C,2.5748945509,2.7182669385,-0.6603027628

$\mathrm{H}, 2.0584700604,3.6836996858,-0.6985078257$

$\mathrm{H}, 2.982296552,2.6009017605,0.3490827914$

$\mathrm{H}, 3.4103962094,2.7653165222,-1.3661886123$

C,2.9321631293,-0.7954689999,3.0512046515

$\mathrm{H}, 1.9688967911,-1.2031516898,3.3682791596$

$\mathrm{H}, 3.7091821646,-1.0956306948,3.7696089917$

$\mathrm{H}, 2.8352241334,0.2938665612,3.0807333308$

$\mathrm{H}, 4.2463838005,-2.1275696808,-0.0874523022$

Zero-point correction $=$

Thermal correction to Energy=

Thermal correction to Enthalpy=

Thermal correction to Gibbs Free Energy=

Sum of electronic and zero-point Energies=

Sum of electronic and thermal Energies=

Sum of electronic and thermal Enthalpies=

Sum of electronic and thermal Free Energies $=$
0.390970 (Hartree/Particle)

$$
0.420473
$$

0.421417

0.329627

$-3313.398820$

$-3313.369318$

$-3313.368374$

$-3313.460164$ 
Table S38. Cartesian coordinates and energy values for the TS15.

Ge,0.2971444147,-1.0572763835,-0.2646622509

$\mathrm{Si}, 3.1131600724,0.7245577178,0.3075763001$

$\mathrm{Si}, 1.4799922424,-0.3185265084,1.5302174027$

$\mathrm{N},-2.7186374997,-0.681623437,0.1561934738$

$\mathrm{N},-1.7690578769,1.152439972,-0.481058582$

C, $1.3285188681,-0.2860387377,-1.7513894299$

$\mathrm{C}, 2.45694939,0.3840946368,-1.4505712538$

C, $-1.5410993485,-0.1793210524,-0.3162288733$

C,-3.6686426357, $0.3301560224,0.306911905$

C,-3.0656935461, $1.4885740749,-0.09230343$

C, $-5.0560929136,0.0840920874,0.8002797139$

C,-3.5973761487,2.8818825393,-0.152908753

$\mathrm{H},-5.0675729541,-0.2964684154,1.8283452203$

$\mathrm{H},-5.5952941247,-0.6351280049,0.172727255$

$\mathrm{H},-5.6257931012,1.0151410113,0.7923193266$

$\mathrm{H},-4.6533330398,2.8945530924,0.1237727461$

$\mathrm{H},-3.5148884775,3.304884979,-1.1605511703$

$\mathrm{H},-3.0694761634,3.5537046057,0.5334221867$

C,3.3556512452,2.6152367433,0.4710633399

$\mathrm{H}, 2.418256192,3.1559460849,0.306617127$

$\mathrm{H}, 3.7104870455,2.8726870945,1.4758390677$

$\mathrm{H}, 4.0977673189,2.9897297113,-0.2458408201$

C,-2.9069763093,-2.0744152248,0.5391996434

$\mathrm{H},-2.7684238466,-2.7346098829,-0.3188591798$

$\mathrm{H},-2.186524552,-2.3444670695,1.3157330342$

$\mathrm{H},-3.9136098389,-2.2090525297,0.9316249664$

C, $-0.7053495168,2.1036911247,-0.7684515626$

$\mathrm{H}, 0.0460781963,2.0214909808,0.0345242337$

$\mathrm{H},-0.2197240155,1.8619491885,-1.7130291387$

$\mathrm{H},-1.1176194936,3.1101805164,-0.8150854938$

$\mathrm{C}, 4.8740998506,-0.0011703165,0.4242801654$

$\mathrm{H}, 5.5528472148,0.4693503845,-0.2985420893$

$\mathrm{H}, 5.2947671471,0.1602035283,1.4236997626$

$\mathrm{H}, 4.863395657,-1.0790447036,0.2397746385$

C,- $0.006505245,-2.941729999,-0.7414758423$

$\mathrm{H},-0.6932799653,-3.0741479444,-1.5838717334$

$\mathrm{H}, 0.9848931196,-3.2996661377,-1.0325175307$

$\mathrm{H},-0.3376109545,-3.5400375218,0.1097870008$

C, $1.1479920496,-0.5658223174,3.4015246519$

$\mathrm{H}, 0.2654632659,-0.0264684497,3.7558322514$

$\mathrm{H}, 1.0464152133,-1.6222194061,3.6661949773$

$\mathrm{H}, 2.0120982101,-0.1737098732,3.9484220113$

C, $0.871239169,-0.5469368489,-3.1662355614$

$\mathrm{H},-0.1552300998,-0.1885009562,-3.3245850829$

$\mathrm{H}, 1.5215937662,-0.0653879259,-3.9062812127$

$\mathrm{H}, 0.8525389676,-1.6234272463,-3.3824092312$

$\mathrm{H}, 3.0577890512,0.7551887964,-2.2924441793$
Zero-point correction $=$
Thermal correction to Energy=
Thermal correction to Enthalpy=
0.396081 (Hartree/Particle)
0.422896
0.423840
Thermal correction to Gibbs Free Energy $=\quad 0.340958$
Sum of electronic and zero-point Energies=
$-3313.479509$
Sum of electronic and thermal Energies=
Sum of electronic and thermal Enthalpies=
$-3313.452694$
$-3313.451750$
Sum of electronic and thermal Free Energies=
$-3313.534631$ 
Table S39. Cartesian coordinates and energy values for the TS16.

Ge,-0.5637304853,-0.3327799645,-0.2056413234

Si,-3.3294409509,0.7866758141,-0.3758046072

$\mathrm{Si},-1.4051746513,1.2435851399,-1.721793703$

$\mathrm{N}, 2.3777796572,-1.0953956362,-0.3295273673$

$\mathrm{N}, 2.1283776452,0.8375501116,0.5838930168$

C, $-1.3743785527,0.1342801767,1.575273898$

C,-2.5955993308,0.6828377584,1.3763474188

C, $1.4316103241,-0.1984267745,0.0515982816$

C,3.661148572,-0.6174933335,-0.0614793242

C,3.5012013826, $0.6077140748,0.5212202669$

C, $4.8944260467,-1.3916605426,-0.3874046163$

$\mathrm{C}, 4.5069638204,1.587408616,1.0265231687$

$\mathrm{H}, 4.944382664,-1.6449863623,-1.4523151068$

$\mathrm{H}, 4.9536307601,-2.3294224898,0.1774726524$

$\mathrm{H}, 5.7832483035,-0.8060510984,-0.1447110183$

$\mathrm{H}, 5.5187981855,1.2210518216,0.8427850391$

$\mathrm{H}, 4.4067333374,1.7548767403,2.1052226939$

$\mathrm{H}, 4.4107026368,2.5604771729,0.5321285491$

C,-4.719673507,2.0873716468,-0.4505704405

$\mathrm{H},-4.3516484281,3.0874393166,-0.1992543234$

$\mathrm{H},-5.1426385804,2.1327327477,-1.4598977335$

$\mathrm{H},-5.5340562479,1.8449506525,0.2429611522$

C,2.0942608424,-2.4098609813,-0.8957111291

$\mathrm{H}, 1.0318823245,-2.6136363046,-0.7513511705$

$\mathrm{H}, 2.3244197929,-2.4349751105,-1.9640771074$

$\mathrm{H}, 2.6874575536,-3.1685443199,-0.37987366$

C, $1.5210345496,2.0687540519,1.0832205728$

$\mathrm{H}, 0.454636334,1.8952012413,1.2212605887$

$\mathrm{H}, 1.9739779728,2.3418192936,2.0386796329$

$\mathrm{H}, 1.6625550726,2.8816914123,0.3661514897$

C,-4.08728245,-0.9209307171,-0.7650960108

$\mathrm{H},-4.9674924969,-1.1151470662,-0.1410526499$

$\mathrm{H},-4.3904529427,-0.9986153861,-1.8142519917$

$\mathrm{H},-3.3443419966,-1.6994561403,-0.5647736226$

C,-0.6644903417,-1.2090836372,-2.6443335009

$\mathrm{H},-0.9836233142,-2.1880784387,-2.3038730217$

$\mathrm{H},-1.1901712676,-0.8937992963,-3.5346933934$

$\mathrm{H}, 0.4139586152,-1.1251494832,-2.7577551315$

C, $-0.9277640222,3.1260581851,-1.4478925163$

$\mathrm{H},-0.975034748,3.4288270261,-0.3944518505$

$\mathrm{H}, 0.0880331369,3.318027623,-1.813296424$

$\mathrm{H},-1.6045145417,3.7740149135,-2.016875989$

C,- $-0.831361735,-0.2286316922,2.9337642168$

$\mathrm{H}, 0.1933752059,0.1372913447,3.0840242986$

$\mathrm{H},-1.4520901766,0.1538194745,3.7544938059$

$\mathrm{H},-0.7815489814,-1.3203241784,3.0402745922$

$\mathrm{H},-3.191804707,0.9842161978,2.2455181582$

Zero-point correction=

Thermal correction to Energy=

Thermal correction to Enthalpy=

Thermal correction to Gibbs Free Energy=

Sum of electronic and zero-point Energies=

Sum of electronic and thermal Energies=

Sum of electronic and thermal Enthalpies=

Sum of electronic and thermal Free Energies=
0.393067 (Hartree/Particle)

0.420897

0.421842

0.336353

$-3313.400419$

$-3313.372588$

$-3313.371644$

$-3313.457133$ 
Table S40. Cartesian coordinates and energy values for the TS17.

Ge,-0.6899522234,-0.2725142753,-0.8132618139

$\mathrm{Si},-2.3548040842,1.369210364,0.1679949393$

$\mathrm{Si},-1.529890771,-0.2862244391,1.5475569568$

$\mathrm{N}, 2.3529197335,-0.9635818763,0.1761801749$

$\mathrm{N}, 2.3514290678,1.0295268213,-0.6046305483$

C, $1.5154316412,0.0136912503,-0.2630068968$

C,3.6911850376, $0.7083047805,-0.360305784$

$\mathrm{C}, 3.6920068577,-0.562504835,0.1365891063$

C, $4.8140468782,1.6519433212,-0.6360888674$

$\mathrm{H}, 4.6873566463,2.6017446356,-0.1037765404$ $\mathrm{H}, 4.9039371575,1.8846301969,-1.7040258781$ $\mathrm{H}, 5.7633559674,1.2178547917,-0.3149400001$

C,4.8183204086,-1.4394531061,0.5722001025

$\mathrm{H}, 5.7662943456,-0.9020523899,0.5001668222$

$\mathrm{H}, 4.9037406598,-2.3393604259,-0.0489255935$

$\mathrm{H}, 4.7026578294,-1.7679773767,1.6115627887$

C,-2.5634979498,-1.7450171641,2.2101940502

$\mathrm{H},-3.1296619665,-1.4328115322,3.0953300827$

$\mathrm{H},-3.2689084583,-2.1035186045,1.4577429857$

$\mathrm{H},-1.9198172235,-2.5817704739,2.5068401975$

C,- $0.4038311838,0.3003663146,2.9801099245$

$\mathrm{H}, 0.161903154,1.1985511069,2.717054752$

$\mathrm{H},-1.0056901576,0.527979746,3.8678416142$

$\mathrm{H}, 0.3174410505,-0.4762330194,3.2626257805$

C,-1.2826400555,2.940986397,0.4592181086

$\mathrm{H},-0.2617292998,2.6809526169,0.7531588809$

$\mathrm{H},-1.2242252291,3.5502882804,-0.4509067097$

$\mathrm{H},-1.7153713238,3.5672676831,1.2485269121$

C,-4.0950206685,2.0981052926,-0.1529378133

$\mathrm{H},-4.2734365,2.9234377636,0.5459934231$

$\mathrm{H},-4.1714546969,2.500868384,-1.1696729903$

$\mathrm{H},-4.8920615228,1.3634250896,-0.016092395$

C, $1.8956519244,2.2867774733,-1.1831952713$

$\mathrm{H}, 0.88037138,2.1438168864,-1.555136369$

$\mathrm{H}, 1.8952869618,3.0899332989,-0.4404721294$

$\mathrm{H}, 2.5443396879,2.571079202,-2.01545754$

C, $1.886240493,-2.2632330864,0.6410707082$

$\mathrm{H}, 1.7902100238,-2.2830545569,1.731189763$

$\mathrm{H}, 0.9112468946,-2.4590951071,0.1943229235$

$\mathrm{H}, 2.5841054183,-3.0433207012,0.3291327886$

C,-2.4464524403,-1.6843472825,-1.3385854851

C,-3.2716476871,-0.7547479293,-1.1768349702

$\mathrm{H},-4.2805911251,-0.3986837912,-1.2633808629$

C,-2.0882461174,-3.0849762806,-1.6536009689

$\mathrm{H},-1.6605198654,-3.5761706487,-0.772623202$

$\mathrm{H},-2.9655368281,-3.6621228362,-1.9740211363$

$\mathrm{H},-1.3340388408,-3.1250029581,-2.4466440203$

Zero-point correction=

Thermal correction to Energy=

Thermal correction to Enthalpy=

Thermal correction to Gibbs Free Energy=

Sum of electronic and zero-point Energies=

Sum of electronic and thermal Energies=

Sum of electronic and thermal Enthalpies=

Sum of electronic and thermal Free Energies=
0.391482 (Hartree/Particle) 0.420458

0.421402

0.332270

$-3313.409768$

$-3313.380792$

$-3313.379848$

$-3313.468981$ 
Table S41. Cartesian coordinates and energy values for the $\mathbf{N}$-Heterocyclic Carbene (NHC).

$\mathrm{N},-2.5011191768,0.6468599098,-0.2494462967$

$\mathrm{N},-1.6367380841,-1.1813418387,0.3903184353$

C,-1.3405930631,0.1528829996,0.2869602256

C,-3.4782391669,-0.3320834997,-0.471595553

C,-2.9224208539,-1.5076751805,-0.0602523999

C,-4.8224387764,-0.032174219,-1.0481815438

$\mathrm{H},-5.4169722007,-0.9453417765,-1.1315655462$

$\mathrm{H},-4.7512571638,0.4063916059,-2.051344969$

$\mathrm{H},-5.3885948913,0.6730319008,-0.4269715616$

C,-3.4677697344,-2.8975092041,-0.0451413381

$\mathrm{H},-4.4859385105,-2.9148891413,-0.4417886606$

$\mathrm{H},-3.5029902913,-3.3146129831,0.9690252087$

$\mathrm{H},-2.8656821715,-3.5824167078,-0.6551710117$

C,-2.6691010615,2.057130859,-0.5473407163

$\mathrm{H},-3.4990099644,2.4906321617,0.0220592142$

$\mathrm{H},-1.741941928,2.5555322251,-0.2658709189$

$\mathrm{H},-2.8574876409,2.221389153,-1.6143078268$

C,- $-0.6848662091,-2.1395137417,0.9210308071$

$\mathrm{H},-0.4329502857,-2.9063147861,0.1797862037$

$\mathrm{H}, 0.2152575132,-1.5839714664,1.1825755704$

$\mathrm{H},-1.0744923387,-2.63728827,1.8161826773$

$\begin{array}{lc}\text { Zero-point correction }= & 0.182617 \text { (Hartree/Particle) } \\ \text { Thermal correction to Energy= } & 0.192761 \\ \text { Thermal correction to Enthalpy= } & 0.193706 \\ \text { Thermal correction to Gibbs Free Energy= } & 0.148083 \\ \text { Sum of electronic and zero-point Energies }= & -383.266717 \\ \text { Sum of electronic and thermal Energies }= & -383.256572 \\ \text { Sum of electronic and thermal Enthalpies }= & -383.255628 \\ \text { Sum of electronic and thermal Free Energies }= & -383.301251\end{array}$

Table S42. Cartesian coordinates and energy values for the Methyl acetylene (Ac).

C,- $-0.4870584784,0.2229299317,-0.0000000002$

C, $0.7203439929,0.2229299512,0.0000000009$

$\mathrm{H}, 1.7854496042,0.2229299683,0.0000000019$

C,-1.9464474464,0.2229299081,-0.0000000016

$\mathrm{H},-2.3417033589,0.7026233229,0.9021721007$

$\mathrm{H},-2.3417033582,0.7643871507,-0.8665127421$

$\mathrm{H},-2.3417033338,-0.7982207683,-0.0356593647$

$\begin{array}{lc}\text { Zero-point correction }= & 0.055568 \text { (Hartree/Particle) } \\ \text { Thermal correction to Energy= } & 0.059550 \\ \text { Thermal correction to Enthalpy= } & 0.060494 \\ \text { Thermal correction to Gibbs Free Energy= } & 0.032389 \\ \text { Sum of electronic and zero-point Energies }= & -116.603859 \\ \text { Sum of electronic and thermal Energies }= & -116.599877 \\ \text { Sum of electronic and thermal Enthalpies }= & -116.598933 \\ \text { Sum of electronic and thermal Free Energies }= & -116.627038\end{array}$

Table S43. Cartesian coordinates and energy values for the 7-Ph.

Ge,1.50886504,0.6723191178,-0.5222785701

Si, $1.3706470911,-0.3576551346,1.6668568907$

Si, $0.138465682,-1.1823452453,-1.2768099112$

$\mathrm{N},-1.1206450269,2.357344966,0.1345532026$

$\mathrm{N}, 0.6398043707,3.4931363957,-0.3493966798$ 
C, $0.1250349191,-1.7818343403,1.4841302834$

$\mathrm{H},-0.1314824782,-2.3771299778,2.3695086503$

C,-0.4441236807,-2.1355062053,0.3021088378

C, $0.1936796002,2.2155160169,-0.1900564912$

C,-1.4964431285,3.7005214468,0.1649541157

C, $-0.3785033136,4.4222007499,-0.1394189578$

C,-2.8866371186,4.1405036383,0.4837727945

C,- $0.1869533432,5.8988112903,-0.2501460329$

$\mathrm{H},-3.1861465542,3.8529780766,1.4984874574$

$\mathrm{H},-3.6186526772,3.709062416,-0.2083978637$

$\mathrm{H},-2.9665522193,5.2269442372,0.4133174569$

$\mathrm{H},-1.1300607405,6.413043062,-0.0550401703$

$\mathrm{H}, 0.1487557671,6.1964613962,-1.2499558442$

$\mathrm{H}, 0.5465196201,6.2742042548,0.4726131131$

C,3.0554057862,-1.1024119323,2.1544194778

$\mathrm{H}, 3.4724742397,-1.7010962266,1.3384068822$

$\mathrm{H}, 3.7759072065,-0.3086714699,2.380837879$

$\mathrm{H}, 2.970104333,-1.7440252056,3.0395722227$

C,-2.0308833056,1.2673577721,0.4564951863

$\mathrm{H},-2.7960917478,1.1611561657,-0.3174226896$

$\mathrm{H},-1.4611499585,0.3439227422,0.5312665043$

$\mathrm{H},-2.519164539,1.462863277,1.4147408853$

C,2.0208715618,3.8274545625,-0.6987203541

$\mathrm{H}, 2.7009539966,3.3878916334,0.031366582$

$\mathrm{H}, 2.2681899872,3.4171898351,-1.6791794011$

$\mathrm{H}, 2.1315784335,4.9116965962,-0.7044031044$

C,0.8196079688,0.7209657351,3.1446016807

$\mathrm{H}, 0.7652947601,0.1184257106,4.05956728$

$\mathrm{H}, 1.5386442174,1.5278619157,3.3249806387$

$\mathrm{H},-0.1625236461,1.1783612135,2.9871273291$

C, 1.4437636358,-2.2485397698,-2.1795030436

$\mathrm{H}, 2.346881766,-2.3232053282,-1.5634237908$

$H, 1.1144005433,-3.2623888424,-2.4297679059$

$H, 1.7394062513,-1.748776954,-3.1089782159$

C,-1.3185688204,-0.9391311761,-2.4850225678

$\mathrm{H},-1.7396657369,-1.903807121,-2.7889265956$

$\mathrm{H},-2.1323381951,-0.3476774842,-2.0548962199$

$\mathrm{H},-0.9737869516,-0.4247560476,-3.3886198027$

C,-1.4556135277,-3.2279432732,0.2435353548

C,-1.4882808814,-4.1499798594,-0.8187954098

C,-2.4340366666,-3.3617655823,1.2479387241

C,-2.43212663,-5.175356247,-0.8599492816

$\mathrm{H},-0.7495518227,-4.0756449585,-1.6094348449$

C,-3.3840428806,-4.3801676506,1.2044788931

$\mathrm{H},-2.4506611812,-2.6448073246,2.0636383652$

C,-3.3866885655,-5.29636957,0.1508319934

$\mathrm{H},-2.4214530818,-5.8816101433,-1.6857387311$

$\mathrm{H},-4.1283468128,-4.4555192138,1.9929013154$

$\mathrm{H},-4.1271205453,-6.09024494,0.1149324835$

Zero-point correction=

Thermal correction to Energy=

Thermal correction to Enthalpy=

Thermal correction to Gibbs Free Energy=

Sum of electronic and zero-point Energies=

Sum of electronic and thermal Energies=

Sum of electronic and thermal Enthalpies=

Sum of electronic and thermal Free Energies=
0.449715 (Hartree/Particle) 0.480483

0.481428

0.388078

$-3505.232322$

$-3505.201553$

$-3505.200609$

$-3505.293958$ 
Table S44. Cartesian coordinates and energy values for the 8-Ph.

$\mathrm{Ge}, 0.686621292,0.7182726541,-1.0250780478$ Si,-1.491713511,3.0142010669, 0.2286819782 Si, $0.7622910392,2.4558246711,0.6666231887$ $\mathrm{N}, 1.1288812101,-1.7040123028,0.9367235572$ $\mathrm{N}, 2.8681200212,-1.148592703,-0.2031219904$ C, $1.5670154328,-0.8033819737,0.0177436147$ C,3.2380654645,-2.253191945,0.5640841176 C,2.1325139792,-2.6124945215,1.2783601421 C,4.6084734797,-2.8426379699,0.5245185669 $\mathrm{H}, 5.3762055873,-2.1057152928,0.7867210849$ $\mathrm{H}, 4.8578081031,-3.2381147472,-0.4671739473$ $\mathrm{H}, 4.6858432169,-3.6672470851,1.2358337882$ C, $1.9178392079,-3.7225947044,2.2522313826$ $\mathrm{H}, 2.8222897772,-4.3274779405,2.3423652472$ $\mathrm{H}, 1.1041978668,-4.3859782266,1.9378965684$ $\mathrm{H}, 1.6694465891,-3.3497469762,3.2528920363$ C, $1.0842028423,1.9734751345,2.4923004877$ $\mathrm{H}, 1.0233068005,2.8556158757,3.1405690201$ $\mathrm{H}, 0.3553075234,1.2435879467,2.8582811595$ $\mathrm{H}, 2.084371887,1.5428265861,2.6158738759$ C,2.0073013984,3.8387425055,0.2336427546 $\mathrm{H}, 1.8756026797,4.1704183075,-0.8008895996$ $\mathrm{H}, 1.8867252867,4.7077083456,0.8904121222$ $\mathrm{H}, 3.0404485992,3.4876332342,0.3388216075$ C,-1.6045400518,4.3182263588,-1.1575273848 $\mathrm{H},-1.0160564319,4.0006656373,-2.0247010409$ $\mathrm{H},-2.6396200556,4.4572498634,-1.4903249142$ $\mathrm{H},-1.2238262109,5.2912082086,-0.8276110883$ C,-2.578533838,3.6010475712,1.6804299874 $\mathrm{H},-2.2466736184,4.5779599095,2.0493759991$ $\mathrm{H},-3.6262060898,3.7018943782,1.3724385821$ $\mathrm{H},-2.5438996687,2.8988664916,2.5197778904$ C,3.7690979533,-0.4233625098,-1.0900515079 $\mathrm{H}, 3.1556583928,0.1513468843,-1.790515953$ $\mathrm{H}, 4.4047279847,0.2644184143,-0.5242284211$ $\mathrm{H}, 4.3984002785,-1.127952269,-1.6376699145$ C,- $0.1993699468,-1.6989093778,1.5368590839$ $\mathrm{H},-0.1135777708,-1.8498431607,2.6153958984$ $\mathrm{H},-0.6570075102,-0.732952461,1.3343314582$ $\mathrm{H},-0.8262701741,-2.4836617496,1.1067377611$ C,-1.2799797536,0.3341771785,-0.7828703311 C,-2.1039717352,1.3514939689,-0.4083283165 $\mathrm{H},-3.1840488652,1.2112564219,-0.5364221009$ C,-1.8357769657,-0.9647487058,-1.2532555189 C,-3.0627528381,-1.4574909677,-0.7624949216 C,-1.1352576363,-1.7773006644,-2.1661849524 C,-3.573845955,-2.6845782325,-1.1792876701 $\mathrm{H},-3.6085993235,-0.8735673374,-0.0275014046$ C,-1.6483014814,-3.0029373318,-2.5888666074 $\mathrm{H},-0.1870336536,-1.4273303304,-2.5642159463$ C,-2.8710147498,-3.465191338,-2.1003906674 $\mathrm{H},-4.5215471141,-3.0359655536,-0.7793944742$ $\mathrm{H},-1.0904818811,-3.5989358151,-3.3065301469$ $\mathrm{H},-3.268153063,-4.4224114211,-2.4262220932$

Zero-point correction= Thermal correction to Energy= Thermal correction to Enthalpy= Thermal correction to Gibbs Free Energy=
0.448995 (Hartree/Particle) 0.479917 0.480861 0.387142

S- 88 
Sum of electronic and zero-point Energies= Sum of electronic and thermal Energies= Sum of electronic and thermal Enthalpies= Sum of electronic and thermal Free Energies=
$-3505.224679$

$-3505.193756$

$-3505.192812$

$-3505.286531$

Table S45. Cartesian coordinates and energy values for the 9-Ph.

Ge,0.3031839923,-1.0290061446,1.193685855

Si,-0.6573365372,2.2520912359,-1.4521325259

Si,-0.7850593144, $0.0061634182,-0.7123549247$

$\mathrm{N}, 3.0144296404,-1.6947083537,-0.2390112836$

$\mathrm{N}, 3.0429563238,0.1456687944,0.8761311303$

C,-2.4606857301,0.8480230077,-0.2183230232

C,-2.3729942327,2.1250020807,-0.6871123842

$\mathrm{C}, 2.2169338284,-0.8676413103,0.4898093301$

C,4.3273981989,-1.2193557104,-0.2962440348

C,4.3401754963,-0.0471110715,0.4019727647

C,5.4216969111,-1.9353521271,-1.0157344131

C,5.4477158531, $0.9157267299,0.6722291402$

$\mathrm{H}, 5.2323153745,-2.0052568993,-2.0935937598$

$\mathrm{H}, 5.5589475407,-2.9550554156,-0.6384328757$

$\mathrm{H}, 6.3676442207,-1.4061038186,-0.8846443553$

$\mathrm{H}, 6.3619005453,0.5923654026,0.1706390488$

$\mathrm{H}, 5.6692814064,0.9975281265,1.7427586046$

$\mathrm{H}, 5.2079081955,1.9215965763,0.309212983$

C, $0.4653077967,3.5420358971,-0.6157189738$

$\mathrm{H}, 0.4692490204,3.4191273865,0.4714237829$

$\mathrm{H}, 1.4974320654,3.4649608321,-0.9778005398$

$\mathrm{H}, 0.1178897022,4.5588853067,-0.8341162501$

C,2.5326147923,-2.8830485967,-0.9295001121

$\mathrm{H}, 2.6046333452,-3.7713212817,-0.2957467831$

$\mathrm{H}, 1.4906295237,-2.7222138887,-1.1970604226$

$\mathrm{H}, 3.1202685081,-3.0425399651,-1.8347174492$

C,2.6161232029,1.299145049,1.6589729059

$\mathrm{H}, 2.4890183883,2.176235863,1.019901398$

$\mathrm{H}, 1.657765696,1.0495785381,2.1252498232$

$\mathrm{H}, 3.3558954369,1.5164845903,2.432828842$

C,-0.6199069886,2.6143492174,-3.3202848917

$\mathrm{H},-0.9698643617,3.6316886081,-3.5322382201$

$\mathrm{H}, 0.3982024017,2.5265570257,-3.7169756424$

$\mathrm{H},-1.2558857958,1.9184767428,-3.8756009879$

C,- $0.1296503236,-2.9840046749,0.9175948879$

$\mathrm{H}, 0.6218310067,-3.5989094727,1.4234710005$

$\mathrm{H},-1.0613762051,-3.1194678271,1.4758899465$

$\mathrm{H},-0.2870333247,-3.3594931887,-0.0962439884$

C,-1.1507478634,-1.1738796995,-2.1848578914

$\mathrm{H},-1.6323704913,-2.0985435872,-1.8485212$

$\mathrm{H},-1.818395352,-0.692546772,-2.9074212156$

$\mathrm{H},-0.228116051,-1.4480807421,-2.7100840535$

$\mathrm{H},-3.1791154203,2.853068791,-0.5550198948$

C,-3.6446445469,0.299809605,0.4826585876

C,-3.5110717143,-0.4744436946,1.6504020677

C,-4.942300054,0.5477615897,-0.0012037071

C,-4.6344618366,-0.9656532371,2.3141295627

$\mathrm{H},-2.5216876897,-0.6616366548,2.0602153277$

C,-6.0639959868,0.0448760749, 0.65605368

$\mathrm{H},-5.061365226,1.1281268728,-0.9116029794$

C,-5.9154051538,-0.712984908,1.8190249323

$\mathrm{H},-4.507997804,-1.5485382107,3.2225052125$

$\mathrm{H},-7.0557689779,0.2430796774,0.2582085029$ 


\section{$\mathrm{H},-6.7886604324,-1.1051637869,2.3326244664$}

Zero-point correction $=$

Thermal correction to Energy=

Thermal correction to Enthalpy=

Thermal correction to Gibbs Free Energy=

Sum of electronic and zero-point Energies=

Sum of electronic and thermal Energies=

Sum of electronic and thermal Enthalpies=

Sum of electronic and thermal Free Energies=
0.448797 (Hartree/Particle)

0.479904

0.480848

0.385397

$-3505.197729$

$-3505.166621$

$-3505.165677$

$-3505.261128$

Table S46. Cartesian coordinates and energy values for the 12-Ph.

Ge,-0.7487094629,-1.3387544675,-1.1612881654

Si, $0.1162143258,-1.6931305487,1.1055589376$

Si, $1.4860513705,-0.29325326,-1.1673140065$

$\mathrm{N},-3.3142906207,-0.1523249292,-0.1545515056$

$\mathrm{N},-2.3346854083,1.2593097364,-1.4571968459$

C,-2.148753215,0.0622606988,-0.8344393561

C,-4.2040512726,0.9105927639,-0.3286715204

C, $-3.5853301133,1.8032915356,-1.1528184285$

C,-5.5479613735, $0.9624957707,0.3186137368$

$\mathrm{H},-6.0327777591,1.9160526974,0.1006585254$

$\mathrm{H},-5.4801674532,0.8677784406,1.408281369$

$\mathrm{H},-6.2132054409,0.1689556281,-0.042228579$

C,-4.0492467156,3.1232333152,-1.6730144623

$\mathrm{H},-5.0269335013,3.3685912876,-1.2538243406$

$\mathrm{H},-4.152897486,3.1251707217,-2.7646498175$

$\mathrm{H},-3.3625203203,3.9338987011,-1.4039566166$

C, $-1.3711146778,1.8416674639,-2.3786658896$

$\mathrm{H},-0.5117351645,2.2467683616,-1.8427474007$

$\mathrm{H},-1.0276641204,1.068683172,-3.070342584$

$\mathrm{H},-1.8501019495,2.6392671747,-2.9458867539$

C,-3.6206681838,-1.3861458235,0.5578689478

$\mathrm{H},-3.2756653623,-2.2356250493,-0.0387992702$

$\mathrm{H},-3.1303791027,-1.4122738003,1.5322732021$

$\mathrm{H},-4.6991483328,-1.4620786954,0.6960148015$

C, $-0.7397915046,-1.2336956159,2.7430766947$

$\mathrm{H}, 0.0280762065,-1.1642058845,3.5213966706$

$\mathrm{H},-1.2363422556,-0.2600876121,2.6885538312$

$\mathrm{H},-1.4716984112,-1.986501612,3.0556920144$

C, $0.7818539543,-3.4673468767,1.2910992681$

$\mathrm{H}, 1.4005421658,-3.5561053578,2.1914382276$

$\mathrm{H},-0.0439231389,-4.1830143026,1.3748046214$

$\mathrm{H}, 1.386989869,-3.7659254326,0.4298191624$

C,2.555797875,-1.6469167091,-1.9815921607

$\mathrm{H}, 2.2073381998,-2.6503219968,-1.7181723644$

$\mathrm{H}, 2.4854479505,-1.5604377124,-3.0722582341$

$\mathrm{H}, 3.6115902929,-1.5591619029,-1.7024095098$

C,2.0480260127,1.376506291,-1.8964023533

$\mathrm{H}, 1.6791046859,2.2245636575,-1.3120634574$

$\mathrm{H}, 3.1410415065,1.4361641485,-1.8916328632$

$\mathrm{H}, 1.710137461,1.4917266881,-2.9327154019$

C, $1.5349388825,-0.4449898879,0.7619711177$

$\mathrm{N}, 1.9715855522,0.3214055145,1.7064758673$

C,2.9275350552,1.3269826965,1.4754378042

C,4.161239691,1.0566106348,0.8563639956

C,2.6923929822,2.6194596208,1.9760865728

C,5.1190803123,2.0616611897,0.720931705

$\mathrm{H}, 4.366519169,0.0468243737,0.5141957059$ 
C,3.6432309749,3.62379295,1.8158524616

$\mathrm{H}, 1.7514645883,2.8146972363,2.4816775285$

C,4.8632448057,3.3515164376,1.1890909198

$\mathrm{H}, 6.0716011341,1.8336613239,0.2496631719$

$\mathrm{H}, 3.4384532965,4.6219856891,2.1938453431$

$\mathrm{H}, 5.6103510266,4.1321805557,1.0810486835$

Zero-point correction=

Thermal correction to Energy=

Thermal correction to Enthalpy=

Thermal correction to Gibbs Free Energy=

Sum of electronic and zero-point Energies=

Sum of electronic and thermal Energies=

Sum of electronic and thermal Enthalpies=

Sum of electronic and thermal Free Energies $=$
0.437384 (Hartree/Particle)

0.468380

0.469324

0.373574

$-3521.266998$

$-3521.236002$

$-3521.235058$

$-3521.330808$

Table S47. Cartesian coordinates and energy values for the 15-Ph.

Ge,-0.4712107142,0.0019574064,-0.9882589633

Si, $1.4340450068,2.9702769295,1.1853569924$

$\mathrm{Si}, 0.1662250614,2.2755564996,-0.4677082146$

$\mathrm{N}, 2.4083202921,-1.2331464238,-1.2453590091$

$\mathrm{N}, 1.540339097,-1.3392932792,0.72430015$

C,-3.1874738227,-2.2316129244,2.1486440051

C,-2.8550633864,-2.9427089062,3.0709523333

C, $1.3195042552,-0.8517317248,-0.5269272606$

C,3.301599186,-1.9644665986,-0.45940152

C,2.7538856489,-2.022731756,0.7911577865

$\mathrm{C}, 4.5813239288,-2.5235063937,-0.9867672775$

C,3.2656565843,-2.6618323008,2.0393069555

H,5.2748887072,-1.7376093377,-1.3091338045

$\mathrm{H}, 4.4145750602,-3.1881623801,-1.8420780138$

$\mathrm{H}, 5.0861190048,-3.1043854625,-0.2123222011$

$\mathrm{H}, 4.228842762,-3.1393887146,1.8490226218$

$\mathrm{H}, 2.5839959777,-3.4328943311,2.416176975$

$\mathrm{H}, 3.4147258521,-1.9294070432,2.840754089$

C,3.1243100743,2.1537143457, 1.5351117369

$\mathrm{H}, 3.1696476608,1.1500814688,1.102505391$

$\mathrm{H}, 3.9458711313,2.7423337822,1.1115267571$

$\mathrm{H}, 3.3056320884,2.0645928697,2.6124553785$

C,2.6335022495,-0.8309928273,-2.6273340146

$\mathrm{H}, 2.1352304992,-1.5084158021,-3.3264516575$

$\mathrm{H}, 2.2354600234,0.1741942997,-2.7588151449$

$\mathrm{H}, 3.7037089659,-0.8281779979,-2.8362600547$

C, $0.6106926547,-1.1329245718,1.8336913538$

$\mathrm{H}, 0.4825698307,-0.0611348204,2.0139054059$

$\mathrm{H},-0.3643666641,-1.558692153,1.5861484774$

$\mathrm{H}, 1.0083473227,-1.6104081038,2.7281410399$

C, $1.4824820676,4.8030488961,1.7153660693$

$\mathrm{H}, 1.6313803925,4.8904683863,2.7976852274$

$\mathrm{H}, 2.2992984722,5.3415199481,1.2223514865$

$\mathrm{H}, 0.5443396984,5.3075018179,1.4676105997$

C, $-0.5433814051,0.0326496223,-3.0105309941$

$\mathrm{H},-0.5606538898,-0.9964006859,-3.384617744$

$\mathrm{H},-1.5364049941,0.4519343101,-3.2038388763$

$\mathrm{H}, 0.191192605,0.6146790224,-3.5704405578$

C,-1.3236650979,3.4440921854,-0.8055596197

$\mathrm{H},-2.2583137931,2.8786152404,-0.7527296683$

$\mathrm{H},-1.3747374201,4.280043276,-0.1024610313$

$\mathrm{H},-1.2444507282,3.8608003527,-1.8159106819$ 
$\mathrm{H},-2.5906985135,-3.5713640148,3.8889930875$

C,-3.5670853425,-1.3899939276,1.0564737071

C,-3.6708257452,0.0008342457,1.2341665141

C,-3.851507868,-1.9462677992,-0.2041617873

C,-4.0558516165,0.8127170321,0.171418064

$\mathrm{H},-3.4485522866,0.4311980161,2.2047112105$

C,-4.2257789071,-1.1249061155,-1.2634226375

$\mathrm{H},-3.771372924,-3.0197746575,-0.3398670109$

C,-4.3291595162,0.2557418729,-1.0796452631

$\mathrm{H},-4.1357879279,1.8853178648,0.318080214$

$\mathrm{H},-4.4384916148,-1.562589732,-2.2342012881$

$\mathrm{H},-4.6242124627,0.8948442948,-1.9065979327$

Zero-point correction=

Thermal correction to Energy=

Thermal correction to Enthalpy=

Thermal correction to Gibbs Free Energy=

35lectronic and zero-point Energies=

Sum of electronic and thermal Energies=

Sum of electronic and thermal Enthalpies=

Sum of electronic and thermal Free Energies $=$
0.445438 (Hartree/Particle)

0.479037

0.479981

0.375880

$-3505.104421$

$-3505.070822$

$-3505.069878$

$-3505.173979$

Table S48. Cartesian coordinates and energy values for the 16-Ph.

$\mathrm{Ge}, 0.4159149261,-1.3997322188,-1.3325828087$

Si,-1.6455593791,2.0053548368,-0.5073295834

Si,-0.7674828476,0.4941642062,-2.0662366068

$\mathrm{N}, 3.1449903179,-0.0355573529,-0.8116327256$

$\mathrm{N}, 2.0119368915,-0.3849166404,0.9881952714$

C,-2.5774856189,1.2754793697,1.076808323

C, $-3.0111536549,2.2768460671,1.8041643248$

C, $1.9623984023,-0.5279544809,-0.36135907$

C,3.9378583647, $0.4114917084,0.2455311909$

C,3.2134747149,0.1988025563,1.3849780499

C,5.2975025982,0.9965814847,0.0534652007

C,3.5362173633,0.4911927059,2.8124330523

$\mathrm{H}, 5.267799381,1.9270026489,-0.5256508953$

$\mathrm{H}, 5.9697890389,0.3042037145,-0.4654812691$

$\mathrm{H}, 5.7468093307,1.2290665797,1.0207912711$

$\mathrm{H}, 4.5039712888,0.990747286,2.886359458$

$\mathrm{H}, 3.58786908,-0.4214980856,3.4171446523$

$\mathrm{H}, 2.787489478,1.1476672209,3.2689002177$

C,- $0.1954749577,3.0522181327,0.1532816686$

$\mathrm{H}, 0.6000889336,2.4227881386,0.5673461397$

$\mathrm{H}, 0.2425950772,3.6610210848,-0.6441060341$

$\mathrm{H},-0.537685299,3.724093949,0.9477318594$

C,3.4822158517,0.0839195308,-2.2247992059

$\mathrm{H}, 3.9312227078,-0.8375821057,-2.6044517149$

$\mathrm{H}, 2.5637841192,0.2926533829,-2.7749932155$

$\mathrm{H}, 4.1834350956,0.9072570814,-2.3624455968$

$\mathrm{C}, 0.9256327726,-0.7418011028,1.9007320127$

$\mathrm{H}, 0.3099493713,0.1301413313,2.1317307219$

$\mathrm{H}, 0.2995669735,-1.4941850535,1.4172758124$

$\mathrm{H}, 1.3432635994,-1.1494429128,2.8225504356$

C,-2.8318811598,3.1634883823,-1.4432234459

$\mathrm{H},-3.2525503738,3.9155425276,-0.7673714214$

$\mathrm{H},-2.307915807,3.6879026211,-2.2485452442$

$\mathrm{H},-3.6675672318,2.611528894,-1.8848896533$

C,1.2819081705,-2.3202218592,-2.8938720201

$\mathrm{H}, 2.0847711129,-2.9655572885,-2.5254060325$ 
$\mathrm{H}, 0.5109740826,-2.9777994012,-3.3050351765$

$\mathrm{H}, 1.6631113349,-1.6882404104,-3.6985231198$

C,-2.0944294133,-0.1716282885,-3.298686982

$\mathrm{H},-2.2598027013,-1.2473767736,-3.1775309653$

$\mathrm{H},-3.0546346627,0.3425888411,-3.196069771$

$\mathrm{H},-1.7445761599,-0.0094088055,-4.3253079945$

$\mathrm{H},-2.9796981247,3.3515402785,1.6311606868$

C,-2.6601266879,-0.1620022777, 1.4471123773

C,-2.7539196449,-1.1877230561,0.4992333829

C,-2.6476236679,-0.522599468,2.8093782683

C,-2.8039210926,-2.5288565887, 0.884546884

$\mathrm{H},-2.7873472915,-0.935508611,-0.5545326754$

C,-2.7060508842,-1.8582575205,3.1980062994

$\mathrm{H},-2.598777321,0.2741620537,3.5456652837$

C, $-2.7766386761,-2.8718058965,2.2358290239$

$\mathrm{H},-2.8607436448,-3.3017575081,0.1236326577$

$\mathrm{H},-2.694779839,-2.1122710492,4.255086347$

$\mathrm{H},-2.8122202379,-3.9145818587,2.5388913544$

\section{Zero-point correction=}

Thermal correction to Energy=

Thermal correction to Enthalpy=

Thermal correction to Gibbs Free Energy=

Sum of electronic and zero-point Energies=

Sum of electronic and thermal Energies=

Sum of electronic and thermal Enthalpies=

Sum of electronic and thermal Free Energies=
0.445493 (Hartree/Particle)

0.477786

0.478730

0.380004

$-3505.091029$

$-3505.058736$

$-3505.057792$

$-3505.156518$

Table S49. Cartesian coordinates and energy values for the 17-Ph.

Ge, $0.7755677579,0.5839008875,1.9688887633$

$\mathrm{Si},-1.2411774061,-2.259528551,-0.6234837324$

$\mathrm{Si},-1.1033575871,-0.6632854464,1.1184295106$

$\mathrm{N}, 3.0220105951,-0.7599906553,0.250804517$

$\mathrm{N}, 2.199251415,1.0344577898,-0.610943065$

C,-2.0104696149,0.2197907671,-0.3463272757

C, $-1.9740132731,-0.6875787561,-1.3679239484$

C,2.0673104933,0.1881354399, 0.4477074662

C,3.7509051848,-0.5118290834,-0.9155299411

C,3.2205616095,0.6200543272,-1.4652727784

C,4.8700434765,-1.3839238459,-1.3795102384

C,3.5807967992,1.3522377746,-2.7151489141

$\mathrm{H}, 4.5315350152,-2.3999222831,-1.6147774414$

$\mathrm{H}, 5.6641471294,-1.4645094886,-0.6285749327$

$\mathrm{H}, 5.3161593362,-0.9715925738,-2.2867062348$

$\mathrm{H}, 4.3827253313,0.8299726249,-3.2405566419$

$\mathrm{H}, 3.9311292576,2.3704606442,-2.5095721323$

$\mathrm{H}, 2.7297403937,1.4280503214,-3.4012505124$

C, $0.3044841128,-2.9363497949,-1.5080421855$

$\mathrm{H}, 1.070148188,-2.1634823863,-1.6273031663$

$\mathrm{H}, 0.7443407726,-3.767454828,-0.9442966063$

$\mathrm{H}, 0.0514989384,-3.3161393566,-2.5051390271$

C, $3.1711634023,-1.9324719777,1.1022182161$

$\mathrm{H}, 3.7534920793,-1.7030270427,1.9981651993$

$\mathrm{H}, 2.1785494386,-2.2676934396,1.4000191684$

$\mathrm{H}, 3.6700546134,-2.7254590299,0.5450752485$

C, $1.3359068659,2.1904132804,-0.8306304245$

$\mathrm{H}, 0.4113892203,1.8958211908,-1.333010459$

$\mathrm{H}, 1.0849061605,2.6139377703,0.1466693656$

$\mathrm{H}, 1.8620720321,2.9305210384,-1.4343173218$ 
C,-2.4898656958,-3.6911562422,-0.4993166899

$\mathrm{H},-2.7503319464,-4.0810676322,-1.490624617$

$\mathrm{H},-2.0769268586,-4.5217219863,0.0844443927$

$\mathrm{H},-3.4149511654,-3.3681623551,-0.0124835448$

C, $1.4120507128,-0.5865017161,3.4898102508$

$\mathrm{H}, 2.4321790337,-0.3066323022,3.7725818146$

$\mathrm{H}, 0.7719035195,-0.2929337221,4.3282876342$

$\mathrm{H}, 1.3533595635,-1.6737512753,3.3908414263$

C,-2.2700160085,-0.9146310149,2.6158902804

$\mathrm{H},-2.3331732606,0.0053834139,3.2077397614$

$\mathrm{H},-3.2787004625,-1.1941595725,2.2966225462$

$\mathrm{H},-1.8884567597,-1.7024256905,3.2744578387$

$\mathrm{H},-2.3550716435,-0.4673799661,-2.3699734635$

C,-2.6212506437, 1.568377225,-0.4232527298

C,-2.2455105653,2.592403987,0.46864032

C,-3.6168767552,1.864153615,-1.3760265419

C,-2.8174683895,3.8623247956,0.3862414066

$\mathrm{H},-1.483414639,2.4032840618,1.2206527938$

C,-4.1917678398,3.1296674749,-1.4530761518

$\mathrm{H},-3.9540839267,1.0818576224,-2.0493238101$

C,-3.7915659815,4.138852625,-0.5733504983

$\mathrm{H},-2.5031139964,4.6364087898,1.0811453256$

$\mathrm{H},-4.9616172464,3.3276396352,-2.1942075599$

$\mathrm{H},-4.2424197828,5.1256489127,-0.6300766596$

Zero-point correction=

Thermal correction to Energy=

Thermal correction to Enthalpy=

Thermal correction to Gibbs Free Energy=

Sum of electronic and zero-point Energies=

Sum of electronic and thermal Energies=

Sum of electronic and thermal Enthalpies=

Sum of electronic and thermal Free Energies=
0.449256 (Hartree/Particle)

0.480279

0.481224

0.386651

$-3505.202204$

$-3505.171180$

$-3505.170236$

$-3505.264808$

Table S50. Cartesian coordinates and energy values for the 18-Ph.

Ge,0.5267383837,-0.1361900534,0.445988522

Si,-0.3607013211,1.9102995852,-0.3709093511

Si,-1.1871614424,-1.5528397025,-0.6177384529

$\mathrm{N}, 2.9002531164,-0.7467726043,-1.2767129176$

$\mathrm{N}, 3.567226273,-0.0307955718,0.6435271774$

C,-2.4294459346,-0.0509073771,-0.3593573393

C,-2.114970379,1.2805132004,-0.3690076856

C, $2.4503390405,-0.3160452998,-0.0729102488$

C,4.2928692486,- $0.7354024327,-1.3198678773$

$\mathrm{C}, 4.7186934244,-0.2878654538,-0.1015207764$

C,5.0644262848,-1.1582152919,-2.5251930842

C, $6.1000956413,-0.0845685348,0.4266814145$

$\mathrm{H}, 4.8004053115,-0.5613338408,-3.4049644819$

$\mathrm{H}, 4.8869950271,-2.2102900487,-2.7750842625$

$\mathrm{H}, 6.1349659745,-1.0363231784,-2.3507827072$

$\mathrm{H}, 6.8336532143,-0.381016266,-0.3252235045$

$\mathrm{H}, 6.2886864897,-0.6845801232,1.3239283411$

$\mathrm{H}, 6.296372336,0.964060349,0.6784932441$

C, $-0.1836785076,3.4674502187,0.7093752635$

$\mathrm{H},-0.5496999302,3.2984886144,1.7267392766$

$\mathrm{H}, 0.8568223897,3.8063005019,0.7722084048$

$\mathrm{H},-0.7684530807,4.2884680684,0.2764666845$

C,2.0532843985,-1.0818727221,-2.4235384881

$\mathrm{H}, 2.4791413579,-1.9373224268,-2.9496479462$ 
$\mathrm{H}, 1.0465818588,-1.3420120971,-2.0765217808$

$\mathrm{H}, 1.994744834,-0.2291854649,-3.1058671191$

C, $3.5443506624,0.545839476,1.9845042338$

$\mathrm{H}, 4.5474300448,0.8755872751,2.2501929896$

$\mathrm{H}, 2.8692274486,1.4026800871,1.9932250163$

$\mathrm{H}, 3.2008163753,-0.186363278,2.7169204595$

C, $0.2689426599,2.3677456798,-2.1108630735$

$\mathrm{H},-0.2522631182,3.2651220297,-2.4645020749$

$\mathrm{H}, 1.3432597804,2.5861560674,-2.1227756125$

$\mathrm{H}, 0.0672196436,1.5649130811,-2.8264286797$

C, $0.371038617,-0.1255445239,2.4163944512$

$\mathrm{H}, 0.8141928409,0.7284361822,2.9305948754$

$\mathrm{H},-0.7148922116,-0.0801794874,2.5338819144$

$\mathrm{H}, 0.7206596414,-1.0600758132,2.8602393408$

C,-1.6258897107,-2.6892561625,0.9122493131

$\mathrm{H},-1.7299287564,-2.172808866,1.8722554801$

$\mathrm{H},-2.5702103859,-3.2080826153,0.7108180511$

$\mathrm{H},-0.857902321,-3.4641316056,1.0260015614$

$\mathrm{H},-2.9406428588,2.0011424347,-0.3817741657$

C,-3.8679373843,-0.4295990466,-0.264979333

C,-4.7076539962,0.1737943613,0.6904113732

C,-4.4323495393,-1.4091311376,-1.1037909387

C,-6.0526264792,-0.176727136,0.79655881

$\mathrm{H},-4.2840741221,0.9141788141,1.3626590644$

C,-5.7795537203,-1.7540282776,-1.0039906342

$\mathrm{H},-3.8041853678,-1.8859110607,-1.8512845033$

C,-6.5977474775,-1.1411920229,-0.0529447151

$\mathrm{H},-6.6758470199,0.3015487273,1.5479810534$

$\mathrm{H},-6.1921905342,-2.5046260003,-1.6731312536$

$\mathrm{H},-7.6459757201,-1.41517723,0.0283116915$

Zero-point correction=

Thermal correction to Energy=

Thermal correction to Enthalpy=

Thermal correction to Gibbs Free Energy=

Sum of electronic and zero-point Energies=

Sum of electronic and thermal Energies=

Sum of electronic and thermal Enthalpies=

Sum of electronic and thermal Free Energies $=$
0.449568 (Hartree/Particle) 0.480205

0.481150

0.387808

$-3505.203590$

$-3505.172953$

$-3505.172009$

$-3505.265350$

Table S51. Cartesian coordinates and energy values for the 19-Ph.

Ge,2.666948063,0.2726027619,-0.9001738897

$\mathrm{Si}, 2.674820719,-0.932720498,1.1960041482$

Si, $0.3312069143,-0.2457244671,-0.8914688361$

$\mathrm{N},-2.1189791392,1.5401272168,-0.8771598782$

$\mathrm{N},-0.4253668306,2.5054485575,0.0450608189$

C, $0.8871280502,-1.5479913284,1.5077908565$

$\mathrm{H}, 0.6542473513,-2.1325075048,2.4077221019$

C, $-0.1235280124,-1.32335872,0.6353165205$

C, $-0.8104558427,1.3475296347,-0.5551512765$

C,-2.5465126671,2.8106759674,-0.5037282581

C, $-1.4753001498,3.4182127636,0.0877632833$

C,-3.9313150307,3.3131973821,-0.7474824208

C,-1.352988486,4.7776745712,0.6928401471

$\mathrm{H},-4.6825097949,2.7345287288,-0.197660165$

$\mathrm{H},-4.1973146286,3.2797441671,-1.8095755104$

$\mathrm{H},-4.0151781387,4.3511125384,-0.4205431422$

$\mathrm{H},-2.3123869339,5.2958985127,0.6448005299$

$\mathrm{H},-0.6169695165,5.396103687,0.1672305037$ 
$H,-1.0549953704,4.7293026767,1.7456062031$ C,3.8020553486,-2.4724672109,1.2533032762 $\mathrm{H}, 3.5571885145,-3.1858737558,0.4605995179$ $\mathrm{H}, 4.8525185331,-2.1858830512,1.1306052205$ $\mathrm{H}, 3.7090907208,-2.9880983136,2.2169324451$ C,-2.9952456541,0.5464898195,-1.4965038608 $\mathrm{H},-2.9200613813,0.5843605788,-2.5860047303$ $\mathrm{H},-2.7250688983,-0.444406404,-1.1414307445$ $\mathrm{H},-4.0233902708,0.7498413289,-1.1993405321$ C, $0.8798880713,2.7598020189,0.6640638941$ $\mathrm{H}, 0.8986681946,2.346745912,1.6758011839$ $\mathrm{H}, 1.6789521662,2.3010336686,0.0653885312$ $\mathrm{H}, 1.0421025945,3.8359306081,0.7105807598$ C,3.1546141222,0.0832856191,2.7451277544 $\mathrm{H}, 3.0659787695,-0.526203626,3.6529315245$ $\mathrm{H}, 4.1945759943,0.4200230004,2.6703546388$ $\mathrm{H}, 2.5302232142,0.9733173564,2.8786987776$ C,3.2296047378,-1.2439548121,-2.1402764893 $\mathrm{H}, 4.3116236849,-1.3550871096,-2.0202507041$ $\mathrm{H}, 2.7630220007,-2.220515411,-1.9828461993$ $\mathrm{H}, 3.0569458188,-0.9247421529,-3.1722432402$ C, $-0.3535753908,-0.9798923482,-2.5175478638$ $\mathrm{H}, 0.384364584,-1.7086693293,-2.8687124222$ $\mathrm{H},-1.3153945323,-1.4941139848,-2.448512206$ $\mathrm{H},-0.4263834036,-0.2074468794,-3.2915896928$ C,-1.5094321125,-1.8088646285,0.8842902266 C,-2.0899990335,-2.8028313065,0.0728295196 C,-2.2906332093,-1.2745265793,1.925412162 C,-3.39381622,-3.2463672736,0.2977911895 $\mathrm{H},-1.4982248405,-3.2482731673,-0.7214890339$ C,-3.5972773271,-1.7121150127,2.1453052358 $\mathrm{H},-1.8572573999,-0.5122775398,2.5668836703$ C,-4.157069257,-2.6986760788,1.3311890445

$\mathrm{H},-3.8121424556,-4.0265255941,-0.3325950694$ $\mathrm{H},-4.1774484295,-1.2854985349,2.9594673175$ $\mathrm{H},-5.1725868102,-3.0425494541,1.5047551624$

Zero-point correction= Thermal correction to Energy= Thermal correction to Enthalpy= Thermal correction to Gibbs Free Energy= Sum of electronic and zero-point Energies= Sum of electronic and thermal Energies= Sum of electronic and thermal Enthalpies= Sum of electronic and thermal Free Energies=
0.449999 (Hartree/Particle) 0.480395

0.481339

0.388875

$-3505.209525$

$-3505.179129$

$-3505.178185$

$-3505.270649$

Table S52. Cartesian coordinates and energy values for the 22-Ph.

Ge,-1.2092285213,-0.772812953,-1.1114138163

Si,-0.7348238887,-1.3571713397,2.9291440366

Si,-1.0399031315,-2.1031264431,0.8892093615

$\mathrm{N},-3.5126191329,1.1246850584,-0.155282703$

$\mathrm{N},-1.521969379,1.8867576414,0.1577223564$

C,3.8092630832,-0.1275219601,-1.4927074979

C,2.6156055014,-0.3361585399,-1.4784980633

C,-2.2012671634,0.7968861167,-0.2860746736

C,-3.6599974405,2.4160529608,0.3550134122

C,-2.3962909175,2.8947154769,0.5610922199

C,-4.9909847116,3.048197871,0.5941220524

C,-1.9379239117,4.2089492419,1.1003369331 
$\mathrm{H},-5.5682740563,2.5184817689,1.3611832011$ $\mathrm{H},-5.5986359442,3.075424361,-0.3173822646$ $\mathrm{H},-4.8634205909,4.077262473,0.9355874334$ $\mathrm{H},-2.7981285167,4.8386503781,1.3355359258$ $\mathrm{H},-1.3169159172,4.7540414041,0.3805858822$ $\mathrm{H},-1.3552386624,4.0912960368,2.0209580446$ C,-1.790415965,0.0634512222,3.6418231413 $\mathrm{H},-2.2870712849,0.621505179,2.8427361105$ $\mathrm{H},-2.5612257085,-0.3145057119,4.3228037085$ $\mathrm{H},-1.1699576782,0.7674687633,4.2083130023$ C,-4.6049131948,0.201618811,-0.4367906782 $\mathrm{H},-4.9141340921,0.2585797762,-1.4841931506$ $\mathrm{H},-4.2617850891,-0.8082230733,-0.2182650908$ $\mathrm{H},-5.4570303879,0.4405036961,0.2004698134$ C, $-0.0619340844,1.9448021185,0.2077211743$ $\mathrm{H}, 0.3168223975,1.1168639189,0.8132717327$ $\mathrm{H}, 0.344599096,1.8502653892,-0.8016697271$ $\mathrm{H}, 0.2464324365,2.8918889677,0.6480170192$ C,- $0.1332698519,-2.494342919,4.334950233$ $\mathrm{H}, 0.552142756,-1.9593743973,5.0020779679$ $\mathrm{H},-0.9702890934,-2.8597985415,4.9398070849$ $\mathrm{H}, 0.4004747646,-3.3602099676,3.9334782426$ C,-2.5994850258,-1.7265885424,-2.2208252529 $\mathrm{H},-2.9838954444,-1.0423495894,-2.9846956585$ $\mathrm{H},-2.0279121111,-2.4947888172,-2.7517359346$ $\mathrm{H},-3.4355117406,-2.215220533,-1.7156493268$ C, $0.0140194557,-3.6595458171,0.4898983847$ $\mathrm{H},-0.6344184402,-4.4598236459,0.1157860777$ $\mathrm{H}, 0.7440018746,-3.4315421417,-0.2932790917$ $\mathrm{H}, 0.5454900018,-4.0438862521,1.3648118603$ $\mathrm{H}, 1.5527239325,-0.5038019223,-1.4704338054$ C,5.2178405529,0.1157983708,-1.4977422446 C,5.8364515291,0.7311654746,-2.6028431074 C, $6.0116635908,-0.2549523288,-0.3952418794$ C,7.2086911952,0.9678632302,-2.6013259678 $\mathrm{H}, 5.2289570985,1.0167354218,-3.4554490408$ C,7.3832481124,-0.0150541336,-0.4017460649 $\mathrm{H}, 5.5389336273,-0.730572536,0.4578223761$ C, $7.986963316,0.596643543,-1.5026604861$ $\mathrm{H}, 7.6726757933,1.4428241811,-3.4609845668$ $\mathrm{H}, 7.983420804,-0.3069525633,0.4552580886$ $H, 9.0570627789,0.7820635967,-1.5047650838$
Zero-point correction $=$
Thermal correction to Energy=
Thermal correction to Enthalpy=
0.446305 (Hartree/Particle) 0.479648 0.480592 Thermal correction to Gibbs Free Energy= Sum of electronic and zero-point Energies= Sum of electronic and thermal Energies= Sum of electronic and thermal Enthalpies= Sum of electronic and thermal Free Energies= 0.374952 $-3505.106831$ $-3505.073488$ $-3505.072544$ $-3505.178183$

Table S53. Cartesian coordinates and energy values for the 23-Ph.

Ge, $0.5365109122,0.787938108,-1.3776670408$ $\mathrm{Si},-0.4306293229,1.8481499977,1.8641961789$ $\mathrm{Si}, 0.5943571098,2.8183290132,-0.0615499556$ $\mathrm{N}, 3.2225340586,-0.2703418866,-0.5424185178$ $\mathrm{N}, 1.674978083,-1.6878659774,-0.0483522153$ C,-2.6209207942,-0.1122619716,0.9640661216 
C, $-1.7760607162,0.6314576543,1.4479123894$

C, $1.894604748,-0.4978486483,-0.6524187378$

C,3.8512015382,-1.3243250048,0.1169634533

C,2.8692397889,-2.2219844113,0.4358722824

C,5.3196950191,-1.3569412468,0.3815901773

C,2.9504391115,-3.5254553086,1.1590364539

$\mathrm{H}, 5.6338980278,-0.5387606038,1.0394802267$

$\mathrm{H}, 5.9032353441,-1.2842022671,-0.5427190321$

$\mathrm{H}, 5.5927915281,-2.2936127747,0.8708305788$

$\mathrm{H}, 3.9869181896,-3.7400942,1.425466529$

$\mathrm{H}, 2.5885325134,-4.3584078247,0.5461313792$

$\mathrm{H}, 2.3683712231,-3.5143621598,2.0871774921$

C, $0.8350229892,0.8827994845,2.9215318022$

$\mathrm{H}, 1.2882979883,0.0605240146,2.3569062006$

$\mathrm{H}, 1.6452479482,1.5526995019,3.2303710711$

$\mathrm{H}, 0.3770437737,0.4619767403,3.8233316255$

C,3.8923294029,0.9235542503,-1.0629405528

$\mathrm{H}, 4.1272092396,0.7987169954,-2.1232895151$

$\mathrm{H}, 3.2340159557,1.7863124228,-0.9197047288$

$\mathrm{H}, 4.8140558422,1.0890909288,-0.5061917634$

C, $0.3487126849,-2.2949730844,0.0999190938$

$\mathrm{H},-0.3175299016,-1.6125754937,0.6314038925$

$\mathrm{H},-0.0744825506,-2.509632608,-0.8826951193$

$\mathrm{H}, 0.4446923518,-3.2220954582,0.6619042072$

C,-1.2148118722,3.1655307345,2.9950752867

$\mathrm{H},-1.6265582441,2.7253957035,3.909910602$

$\mathrm{H},-0.4625875107,3.908958793,3.2821924052$

$\mathrm{H},-2.0238431831,3.6941466889,2.4812067957$

C, $1.0879224955,1.0681691019,-3.2531189952$

$\mathrm{H}, 1.4143343537,0.1483837089,-3.7446771901$

$\mathrm{H}, 0.2004436802,1.4340938527,-3.7772037576$

$\mathrm{H}, 1.8655890454,1.8313572672,-3.3270079853$

C, $-0.9667085647,3.5826425603,-0.9923352455$

$\mathrm{H},-0.6915101943,3.8315911329,-2.0242874152$

$\mathrm{H},-1.8484854995,2.9338963638,-1.0343144151$

$\mathrm{H},-1.2602455107,4.5199929155,-0.5054607744$

$\mathrm{H},-0.6427389202,-0.1903394798,-1.541250006$

C,-3.5714689818,-0.9712667314,0.3323669399

C,- $-4.6510714661,-1.5328583428,1.0409543438$

C,-3.4302173506,-1.2677673469,-1.0394995228

C,-5.5597899712,-2.3667235809,0.3934303618

$\mathrm{H},-4.7653870597,-1.305194994,2.0958203078$

C,- $-4.3417153806,-2.1039165981,-1.6773498526$

$\mathrm{H},-2.5918071113,-0.8283846102,-1.5699829049$

C,-5.4095793434,-2.6560104572,-0.9650055831

$\mathrm{H},-6.3896838573,-2.7922611046,0.9504838207$

$\mathrm{H},-4.2217351103,-2.3217671998,-2.7349475536$

$\mathrm{H},-6.1218145294,-3.3053215593,-1.465795639$

Zero-point correction=

Thermal correction to Energy=

Thermal correction to Enthalpy=

Thermal correction to Gibbs Free Energy=

Sum of electronic and zero-point Energies=

Sum of electronic and thermal Energies=

Sum of electronic and thermal Enthalpies=

Sum of electronic and thermal Free Energies=
0.446051 (Hartree/Particle)

0.478095

0.479039

0.381310

$-3505.170783$

$-3505.138739$

$-3505.137795$

$-3505.235523$ 
Table S54. Cartesian coordinates and energy values for the 30-Ph.

Ge, $0.2677735919,0.2172883612,-0.407386032$ $\mathrm{Si}, 0.805636036,2.5280225036,0.2718647012$ $\mathrm{Si},-0.0758340935,1.0842735674,1.8551151321$ $\mathrm{N}, 2.3684750736,-1.7862489178,0.3905712896$ $\mathrm{N}, 3.1986502995,-0.407181143,-1.0448510284$ C,2.0812607753,-0.6430278946,-0.2962258348 $\mathrm{C}, 4.1679955147,-1.3900815966,-0.8309852726$ C,3.6467126485,-2.2595996759,0.0809208055 C, $5.4880778874,-1.3772737031,-1.526469996$ $\mathrm{H}, 6.0697747301,-0.4793423597,-1.2860550687$ $\mathrm{H}, 5.3752175364,-1.4122001344,-2.6162987441$ $\mathrm{H}, 6.0826506458,-2.2433555291,-1.2293034624$ C,4.2343896197,-3.4873257014,0.6928616275 $\mathrm{H}, 5.2513737896,-3.6392076658,0.3256889964$ $\mathrm{H}, 3.6594104991,-4.3877215796,0.4456966902$ $\mathrm{H}, 4.285348384,-3.4172793602,1.7855803716$ C,-1.9176150592,1.2155416189,2.3203151065 $\mathrm{H},-2.0750960186,1.9725574909,3.0966789383$ $\mathrm{H},-2.515004549,1.4845936099,1.4445926702$ $\mathrm{H},-2.2973930911,0.2586220383,2.6968501106$ C, $0.9435427551,0.7278565841,3.4343039356$ $\mathrm{H}, 2.0176255708,0.6942009193,3.2270159682$ $\mathrm{H}, 0.767871442,1.514033349,4.1776002145$ $\mathrm{H}, 0.6552514438,-0.2257379174,3.8924505321$ C,2.6065737693,3.1045376082,0.5632595423 $\mathrm{H}, 3.1945101483,2.3334978236,1.0703465079$ $\mathrm{H}, 3.1111581775,3.3477656905,-0.378992216$ $\mathrm{H}, 2.6214113676,4.0051743297,1.1875336501$ C,- $0.1827688161,3.9593515169,-0.5108273974$ $\mathrm{H},-0.0809719197,4.8718042849,0.0879082531$ $\mathrm{H}, 0.1723970525,4.1812657296,-1.5242901153$ $\mathrm{H},-1.2469702199,3.7146555341,-0.569598474$ C,3.3019911723,0.6360191972,-2.0538257724 $\mathrm{H}, 2.5166121324,1.3673972664,-1.863778588$ $\mathrm{H}, 4.2788116245,1.1211788542,-1.9968235581$ $\mathrm{H}, 3.1629243559,0.2181420739,-3.0565076926$ C,1.4138093238,-2.4364001136,1.2771396034 $\mathrm{H}, 1.2979044203,-1.8706888708,2.2039236295$ $\mathrm{H}, 0.4425619701,-2.4829962231,0.777079034$ $\mathrm{H}, 1.7578226988,-3.4458694993,1.5023818281$ C,-3.5079813096,0.7074712402,-1.5151199167 C,-2.8158580303,1.5797158558,-1.9897893402 $\mathrm{H},-2.1915713127,2.3302670217,-2.4147539292$ C,-4.2843615311,-0.3479604782,-0.9427686272 C, $-5.6779863425,-0.4033625253,-1.1277884179$ C,-3.6501693249,-1.3519454847,-0.1854243634 C,-6.419482564,-1.4402649181,-0.566831269 $\mathrm{H},-6.1659726278,0.3701304641,-1.7120502692$ C,-4.4015562841,-2.3832188986,0.3717528278 $\mathrm{H},-2.57400243,-1.3010330289,-0.0499101899$ C,-5.7851489305,-2.4321680325,0.1842749171 $\mathrm{H},-7.4948796227,-1.4743468412,-0.715747924$ $\mathrm{H},-3.9053140125,-3.1534766523,0.9554767274$ $\mathrm{H},-6.3664208766,-3.2390945185,0.6211226487$

Zero-point correction= Thermal correction to Energy= Thermal correction to Enthalpy= Thermal correction to Gibbs Free Energy=
0.446260 (Hartree/Particle) 0.479174 0.480119 0.379467 
Sum of electronic and zero-point Energies= Sum of electronic and thermal Energies= Sum of electronic and thermal Enthalpies= Sum of electronic and thermal Free Energies=
$-3505.146971$ $-3505.114057$

$-3505.113113$

$-3505.213764$

Table S55. Cartesian coordinates and energy values for the 31-Ph.

Ge, 1.0263672924,-0.2216691867,1.4447730831 Si,-3.7764329894,-1.7788470926,-1.7559390651 Si, $0.2227211427,-2.3254865788,1.1328910489$ $\mathrm{N}, 3.9764731472,0.0486294976,0.8562327775$ $\mathrm{N}, 2.7811614104,0.1380703246,-0.9401993734$ C,-3.9458987756,0.0169106149,-1.4456729529 C,-4.9116495872,-0.446776336,-2.2660568256 C,2.6915498956,0.0199891123,0.4105798165 $\mathrm{C}, 4.8702244189,0.1800447921,-0.2074294761$ C,4.1129397627,0.2295960555,-1.3438056591 C, $6.3503495727,0.2428621748,-0.0257605865$ C, $4.5149577044,0.3542233832,-2.7756289935$ $\mathrm{H}, 6.7532521518,-0.6796072236,0.4087774748$ $\mathrm{H}, 6.6463993663,1.0732774455,0.6251807709$ $\mathrm{H}, 6.8409025489,0.389542381,-0.9898284326$ $\mathrm{H}, 5.6029956151,0.3537389424,-2.8647466536$ $\mathrm{H}, 4.1479741745,1.2829436757,-3.2280154662$ $\mathrm{H}, 4.1329041022,-0.4787751839,-3.3760124174$ C,-2.4518646052,-2.4596937904,-2.9139817761 $\mathrm{H},-2.2063508242,-1.7364385188,-3.6967533099$ $\mathrm{H},-1.5404920005,-2.6886590127,-2.3495173441$ $\mathrm{H},-2.7847688376,-3.3862110862,-3.3951800231$ C,4.3302918303,-0.1920632791,2.2493298854 $\mathrm{H}, 4.135565452,0.6894325397,2.864274628$ $\mathrm{H}, 3.7193438106,-1.023535387,2.6148542269$ $\mathrm{H}, 5.38617291,-0.4522513404,2.3176858684$ C,1.6319077713,0.1473356385,-1.8405439155 $\mathrm{H}, 1.4101667698,-0.8601040088,-2.2004141407$ $\mathrm{H}, 0.7680391287,0.5148940742,-1.2814610732$ $\mathrm{H}, 1.8363486397,0.8034167638,-2.688500803$ C,-4.2744069437,-3.0689795895,-0.4743968693 $\mathrm{H},-4.7011136702,-3.9558841607,-0.9565487305$ $\mathrm{H},-3.4021183831,-3.3952263665,0.1025786258$ $\mathrm{H},-5.0175842584,-2.6659128654,0.2188389236$ C, $1.2090829396,0.8907621042,3.0804062045$ $\mathrm{H}, 1.669561255,1.8570637531,2.8593907668$ $\mathrm{H}, 0.1935494109,1.076758257,3.4391376089$ $\mathrm{H}, 1.7676278498,0.3902036787,3.8750098539$ C,-0.9945725373,-2.3731253228,2.6715822575 $\mathrm{H},-1.1478886133,-1.4239031225,3.1937381768$ $\mathrm{H},-1.975857999,-2.7545475383,2.3659007664$ $\mathrm{H},-0.5957436772,-3.092489932,3.3999992472$ $\mathrm{H},-5.7561880311,0.0048277057,-2.7822141764$ C,-3.5430625229, $1.2941288439,-0.8590711012$ C, $-2.3932446437,1.3607527141,-0.0562365916$ C,-4.2744478451,2.4744957992,-1.0854248493 C,-1.9785581217,2.5698534513,0.4985639014 $\mathrm{H},-1.8133619692,0.4617854619,0.1313132568$ C,-3.8645629188,3.6814939205,-0.5247690536 $\mathrm{H},-5.1672402609,2.4354858829,-1.7038077538$ C,-2.7136783297,3.734164493,0.268790082 $\mathrm{H},-1.077497565,2.5893415417,1.1059401668$ $\mathrm{H},-4.4406496226,4.5850186686,-0.7056067487$ 


\section{$\mathrm{H},-2.3958135406,4.6781372307,0.703058743$}

Zero-point correction $=$

Thermal correction to Energy=

Thermal correction to Enthalpy=

Thermal correction to Gibbs Free Energy=

Sum of electronic and zero-point Energies= Sum of electronic and thermal Energies=

Sum of electronic and thermal Enthalpies=

Sum of electronic and thermal Free Energies=
0.446892 (Hartree/Particle)

0.479581

0.480526

0.376032

$-3505.117075$

$-3505.084385$

$-3505.083441$

$-3505.187935$

Table S56. Cartesian coordinates and energy values for the 32-Ph.

$\mathrm{Ge},-1.4976812536,-1.2381357673,-1.1477207159$

Si, $1.0937507279,-1.0920688495,1.2949068244$

Si,-0.8569969408,-2.3245947434,0.8828090229

$\mathrm{N},-3.6124604868,0.5341054183,0.2457690946$

$\mathrm{N},-2.0126219688,1.7066316878,-0.5900660447$

C,2.911394871, $0.0094983144,-0.5284397592$

C, $1.7440405564,-0.4967286259,-0.4805907728$

C, $-2.4504778869,0.4291424712,-0.4475942515$

C,-3.9073074521, $1.8649548221,0.537233019$

C,-2.8872654317, 2.6086377976,0.0140283748

C,-5.1282102832,2.2827218368,1.2873009952

C,-2.6492734057,4.0819779789,0.0254101734

$\mathrm{H},-5.1377350795,1.8856655886,2.3089064455$

$\mathrm{H},-6.0453714812,1.9449838325,0.7921722461$

$\mathrm{H},-5.1727126155,3.3711526613,1.35849213$

$\mathrm{H},-3.4378054486,4.5873509456,0.5861791254$

$\mathrm{H},-2.6403963265,4.505031591,-0.9858290376$

$\mathrm{H},-1.6931649659,4.3345201694,0.4970327981$

C, $0.7751270498,0.5104411271,2.2800254228$

$\mathrm{H},-0.0478957322,1.0866546463,1.842556366$

$\mathrm{H}, 0.5031588193,0.2824646957,3.3158863312$

$\mathrm{H}, 1.6682037013,1.1451268628,2.2935963276$

C,-4.4250401505,-0.6077577226,0.6538809286

$\mathrm{H},-5.1656062866,-0.8518794705,-0.112483132$

$\mathrm{H},-3.7563137757,-1.4564081687,0.8126599161$

$\mathrm{H},-4.9367021811,-0.3728559472,1.5877170305$

C,- $0.772783414,2.0922349424,-1.2601974281$

$\mathrm{H}, 0.0227824576,2.2699796817,-0.5322967199$

$\mathrm{H},-0.4727420113,1.2718870646,-1.9148473822$

$\mathrm{H},-0.9406895245,2.9959529533,-1.8489319109$

C,2.4954328278,-2.0264036826,2.1778069877

$\mathrm{H}, 3.4050615059,-1.4194191728,2.235795028$

$\mathrm{H}, 2.1934673832,-2.2817265624,3.1993914134$

$\mathrm{H}, 2.7428884102,-2.9576803158,1.6591296829$

C,-2.9963340977,--2.1448424921,-2.1187532546

$\mathrm{H},-3.4257790177,-1.4675072439,-2.862522346$

$\mathrm{H},-2.5468375856,-2.9812516882,-2.6611789435$

$\mathrm{H},-3.7941734272,-2.5377221313,-1.4827803657$

C,- $0.2554716422,-4.0563206359,0.2240788515$

$\mathrm{H},-1.1147380193,-4.7125910556,0.0468768928$

$\mathrm{H}, 0.3012076104,-3.9792445351,-0.7163992425$

$\mathrm{H}, 0.3871984087,-4.5496084204,0.9609085164$

$\mathrm{H}, 0.9780562046,-0.6201712499,-1.2898537697$

C,4.2029719241,0.5172747549,-0.4398288029

$\mathrm{C}, 4.4370804617,1.8917191917,-0.1377125783$

C, $5.3401124044,-0.3148017576,-0.6625423862$

C, $5.7286067571,2.3891014064,-0.0567303269$ 
$H, 3.5852494898,2.5428611169,0.0312538506$

C,6.6227984221,0.2057235183,-0.5834177941

$\mathrm{H}, 5.182932008,-1.3626964888,-0.8957665715$

C,6.8330441781,1.5568054744,-0.2790617409

$\mathrm{H}, 5.881602435,3.438771577,0.1796506539$

$H, 7.4734342048,-0.447665013,-0.7574641038$

$\mathrm{H}, 7.8409676832,1.9547815018,-0.2168444385$

Zero-point correction=

Thermal correction to Energy=

Thermal correction to Enthalpy=

Thermal correction to Gibbs Free Energy=

Sum of electronic and zero-point Energies=

Sum of electronic and thermal Energies=

Sum of electronic and thermal Enthalpies=

Sum of electronic and thermal Free Energies=
0.445154 (Hartree/Particle) 0.477676

0.478620

0.377054

$-3505.116852$

$-3505.084330$

$-3505.083386$

$-3505.184952$

Table S57. Cartesian coordinates and energy values for the 33-Ph.

Ge,-0.1191153611,-0.2705525982,0.9165258851

$\mathrm{Si},-0.1465072406,2.1247882253,1.1140018837$

Si, $1.0197184646,3.116123641,-0.4168210323$

$\mathrm{N},-3.223403051,-0.7961948268,1.2111376169$

$\mathrm{N},-2.564581335,-0.3729143357,-0.7952826011$

C, $-2.1172293621,-0.4697182681,0.4880899229$

C, $-4.3548112318,-0.8894784391,0.3948322941$

C, $-3.9359454346,-0.61388528,-0.8744536383$

C, $-5.7130831538,-1.2358808292,0.9089220971$

$\mathrm{H},-6.4282810657,-1.2654144448,0.08454008$

$H,-6.0815312873,-0.5009129185,1.6341834708$

$\mathrm{H},-5.731253588,-2.2188006686,1.3938981263$

C,-4.6969260837,-0.5618231544,-2.1573728813

$\mathrm{H},-5.7569838208,-0.7497065411,-1.9758058844$

$\mathrm{H},-4.348017713,-1.3144785487,-2.8742885476$

$\mathrm{H},-4.612747489,0.4179847489,-2.6408746849$

C,-1.7161372936,0.0145394077,-1.9158284124

$\mathrm{H},-1.5947253885,1.1018380762,-1.9491620056$

$\mathrm{H},-0.729328586,-0.431133113,-1.7632653441$

$\mathrm{H},-2.1545881844,-0.3457445077,-2.8469232169$

C,-3.228675245,-0.9087249367,2.6646946883

$\mathrm{H},-4.2398592795,-1.1282117017,3.0046358933$

$\mathrm{H},-2.562543723,-1.7066611357,2.9926953536$

$\mathrm{H},-2.8972166001,0.0309769927,3.1104031819$

C,-1.2543739175,3.2414092456,2.2167905226

$\mathrm{H},-0.9946679001,4.2942557987,2.0687507691$

$\mathrm{H},-2.3182123807,3.1160442575,1.9903102195$

$\mathrm{H},-1.1034155875,3.0042955575,3.2752942124$

C, $0.0185173511,-0.8886925304,2.8300957844$

$\mathrm{H}, 1.0706427407,-0.7168780515,3.0730638265$

$\mathrm{H},-0.5918049239,-0.3849447142,3.5818768697$

$\mathrm{H},-0.1524420618,-1.9694346186,2.8817073981$

C,2.1245636793,2.0092889274,-1.4919155458

$H, 1.8826950842,0.9767400683,-1.2209896398$

$H, 1.9464538829,2.1480904951,-2.5638855168$

H,3.1896830409,2.1784691108,-1.3018227764

C, $1.3611444387,4.9769784762,-0.6093286633$

$\mathrm{H}, 0.7287384778,5.5607354908,0.0657731959$

$\mathrm{H}, 2.407089707,5.2077140978,-0.3791642828$

$\mathrm{H}, 1.1632488054,5.3121084821,-1.6334627851$

C,3.9520030415,-1.9361070573,-0.1578122797 
C,2.8957359623,-2.4555563086,-0.9175740199

C,5.2719153784,-2.3465072806,-0.383092293

C,3.1752700835,-3.3930640105,-1.9090305258

$\mathrm{H}, 1.8858678357,-2.1085655975,-0.7153551644$

C,5.5323542914,-3.2854852449,-1.3786272351

$\mathrm{H}, 6.0702000591,-1.9282541111,0.2203374142$

C,4.487736395,-3.8105586371,-2.1424978923

$\mathrm{H}, 2.3617196347,-3.8003306711,-2.5021173215$

$\mathrm{H}, 6.5539153245,-3.6071134536,-1.5569566167$

$\mathrm{H}, 4.6965451103,-4.5425523866,-2.9168773211$

C,3.3804270929,-0.224174896,1.6644308017

N,3.6798934072,-1.0072462818,0.8334226205

Zero-point correction $=$

Thermal correction to Energy=

Thermal correction to Enthalpy=

Thermal correction to Gibbs Free Energy=

Sum of electronic and zero-point Energies= Sum of electronic and thermal Energies=

Sum of electronic and thermal Enthalpies=

Sum of electronic and thermal Free Energies=
0.435348 (Hartree/Particle)

0.468393

0.469337

0.365736

$-3521.183898$

$-3521.150853$

$-3521.149909$

$-3521.253510$

Table S58. Cartesian coordinates and energy values for the 34-Ph.

Ge,-0.3147420436,-0.2365261766,-0.114984405

$\mathrm{Si},-0.3592889624,2.1156830748,0.6632450479$

Si, $1.5685054959,2.6908025631,-0.4855079347$

$\mathrm{N},-3.211856863,-1.2458347419,0.6249937985$

$\mathrm{N},-3.0941954365,0.0097813893,-1.1207511919$

C,-2.3306665514,-0.5209237196,-0.1224341376

C,-4.5112343068,-1.150665148,0.1147425532

C,-4.4337943965,-0.3562143667,-0.990390215

C,-5.6847053469,-1.8322247011,0.7368761595

$\mathrm{H},-6.593490549,-1.5974150594,0.1790685032$

$\mathrm{H},-5.8453434768,-1.5141503015,1.7736417219$

$\mathrm{H},-5.5761094255,-2.9236144034,0.7393587094$

C,-5.4982303895,0.0876350103,-1.9378507162

$\mathrm{H},-6.4691360383,-0.2974419663,-1.6196621457$

$\mathrm{H},-5.3208130762,-0.2732427542,-2.9579073747$

$\mathrm{H},-5.5752280493,1.1800543219,-1.9821468137$

C,-2.5455400605,0.8562272662,-2.1726001412

$\mathrm{H},-2.1880467331,1.7969325655,-1.7440906478$

$\mathrm{H},-1.6964686028,0.3444412817,-2.6366219061$

$\mathrm{H},-3.3153789581,1.052907318,-2.9182542088$

C,-2.8705497804,-1.9134576012,1.8726973309

$\mathrm{H},-3.6388543766,-2.6486653504,2.1124212103$

$\mathrm{H},-1.9152999884,-2.4221724788,1.7694988766$

$\mathrm{H},-2.7998204962,-1.1928856083,2.6931916112$

C,0.0409351623,2.1960183957,2.5668958294

$\mathrm{H}, 0.3845505828,3.1975595708,2.8501475466$

$\mathrm{H},-0.8838196909,2.007657585,3.1257233397$

$\mathrm{H}, 0.7820614569,1.4685142129,2.9135986672$

C,0.2921358309,-1.1840612909,1.565696665

$\mathrm{H}, 1.3219888834,-0.8452988668,1.7119814306$

$\mathrm{H},-0.2635025638,-0.9524584421,2.4764615176$

$\mathrm{H}, 0.3369489721,-2.2699782647,1.426511803$

C, $1.3128053517,2.4852985724,-2.3593999056$

$\mathrm{H}, 0.9768856152,1.4559533781,-2.5275409014$

$\mathrm{H}, 0.5276009332,3.1666125248,-2.7036113046$

$H, 2.2183360131,2.6705634593,-2.9450959986$ 
C,2.5624983525,4.2586741846,-0.0852091664 $\mathrm{H}, 2.9094701506,4.2615838726,0.9529095255$ $\mathrm{H}, 3.4285608135,4.386647175,-0.7419237422$ $\mathrm{H}, 1.9063855832,5.1267263304,-0.2121687864$ C,3.7123381377,-1.1178395812,0.0179388945 C,3.0586177742,--2.137759352,-0.6991317985 C, $4.8354077748,-1.4000569434,0.8140316738$ C,3.5487764515,-3.4350532197,-0.6157984323 $\mathrm{H}, 2.1644036643,-1.8900044185,-1.2636347149$ C,5.2999780775,-2.7100756332,0.8877176529 $\mathrm{H}, 5.3238622661,-0.5974579353,1.35561167$ C,4.6663851983,-3.7296517812,0.173562131 $\mathrm{H}, 3.0490343279,-4.2260831608,-1.1667151792$ $\mathrm{H}, 6.1657685794,-2.9331203152,1.5037887432$ $\mathrm{H}, 5.0349472063,-4.7485832456,0.2372211162$ C,2.8608251365,1.2620434955,-0.3028366971 $\mathrm{N}, 3.2437733706,0.1662782799,-0.0834392626$
Zero-point correction=
Thermal correction to Energy=
Thermal correction to Enthalpy=
0.436435 (Hartree/Particle)
0.468226
0.469170
Thermal correction to Gibbs Free Energy=
Sum of electronic and zero-point Energies=
Sum of electronic and thermal Energies=
Sum of electronic and thermal Enthalpies=
0.371970
$-3521.194722$
$-3521.162931$
$-3521.161987$
Sum of electronic and thermal Free Energies $=$
$-3521.259188$

Table S59. Cartesian coordinates and energy values for the 14-Ph.

$\mathrm{Ge},-1.076114,-0.590004,-1.195696$

$\mathrm{Si}, 0.234595,-0.100422,0.780167$

Si, 1.73559,1.638348,0.325093

$\mathrm{N},-3.906287,-1.056454,0.10604$

$\mathrm{N},-3.415224,1.003777,-0.288665$

C,-2.893966, $-0.25654,-0.328514$

C, $-5.051136,-0.312574,0.403548$

C, $-4.735275,0.993065,0.164288$

C,-6.316252,-0.937088,0.890537

$\mathrm{H},-7.089351,-0.176488,1.015819$

$\mathrm{H},-6.185568,-1.434519,1.859077$

$\mathrm{H},-6.699348,-1.68435,0.186265$

C,-5.555795,2.229996,0.320645

$\mathrm{H},-6.541286,1.978474,0.717383$

$\mathrm{H},-5.708535,2.747699,-0.633558$

$\mathrm{H},-5.093332,2.942056,1.013556$

C,-2.661671,2.192448,-0.670494

$\mathrm{H},-1.919619,2.442708,0.092271$

$\mathrm{H},-2.134881,1.984421,-1.607137$

$\mathrm{H},-3.34625,3.02955,-0.803989$

C,-3.794886, $-2.497856,0.27583$

$\mathrm{H},-4.195016,-3.032886,-0.591175$

$\mathrm{H},-2.743826,-2.745898,0.392632$

$\mathrm{H},-4.342122,-2.807816,1.16812$

C, $-0.13663,-0.698845,2.559534$

$\mathrm{H}, 0.691139,-0.43972,3.22693$

$\mathrm{H},-1.051637,-0.240249,2.948389$

$\mathrm{H},-0.260878,-1.786645,2.581083$

C, $-0.873949,-2.597588,-1.294458$

$\mathrm{H}, 0.085025,-2.712889,-1.809686$

$\mathrm{H},-0.818551,-3.176754,-0.368642$ 
$\mathrm{H},-1.636627,-3.028178,-1.951804$

C, $1.518854,2.550342,-1.325671$

$\mathrm{H}, 0.934081,1.930118,-2.011717$

$\mathrm{H}, 0.991323,3.500614,-1.183638$

$\mathrm{H}, 2.488605,2.768973,-1.786531$

C,2.739646,2.66157, 1.568331

$\mathrm{H}, 2.865032,2.122281,2.511476$

$\mathrm{H}, 3.73604,2.883029,1.170722$

$\mathrm{H}, 2.239944,3.612292,1.784792$

C,2.084232,-0.23626,0.310681

$\mathrm{N}, 3.011544,-1.113612,0.456754$

C, $4.355562,-0.785517,0.170823$

C,4.739893,-0.015598,-0.941181

C,5.35287,-1.322104,1.001406

C,6.089011,0.227773,-1.195942

$\mathrm{H}, 3.974289,0.356741,-1.614246$

C,6.697303,-1.055288, 0.755674

$\mathrm{H}, 5.045869,-1.938336,1.840855$

C,7.073039,-0.280122,-0.345156

$\mathrm{H}, 6.373697,0.81156,-2.067472$

$\mathrm{H}, 7.456613,-1.463577,1.417185$

H,8.122883,-0.087914,-0.546536

Zero-point correction=

Thermal correction to Energy=

Thermal correction to Enthalpy=

0.436304 (Hartree/Particle)

0.467865

0.468809

Thermal correction to Gibbs Free Energy $=\quad 0.370552$

Sum of electronic and zero-point Energies $=\quad-3521.212626$

Sum of electronic and thermal Energies $=\quad-3521.181066$

Sum of electronic and thermal Enthalpies $=\quad-3521.180121$

Sum of electronic and thermal Free Energies $=\quad-3521.278378$

Table S60. Cartesian coordinates and energy values for the 35-Ph.

Ge,1.2108928882,-0.592330793,0.2725296387

$\mathrm{Si},-0.6104906611,-2.1237151891,0.5964770584$

$\mathrm{Si},-0.6627726045,0.8837949877,0.536864891$

$\mathrm{N}, 2.8612058989,1.0342977843,-1.7465135326$

$\mathrm{N}, 1.6048338019,-0.4227529395,-2.7134612476$

C, $1.9784083103,0.0342475784,-1.4966539816$

C,3.0517942144,1.2009222796,-3.1176540259

C,2.2484117822,0.2796272612,-3.7307889707

C,3.9757472053,2.222317242,-3.6933322984

$\mathrm{H}, 3.9991993617,2.1369366878,-4.7811357307$

$\mathrm{H}, 5.0016420241,2.0952680553,-3.3302901508$

$\mathrm{H}, 3.6609290077,3.2444230226,-3.4526922842$

C,2.0318037795,0.0044442301,-5.1821383938

$\mathrm{H}, 2.6362848911,0.683773718,-5.7858823184$

$\mathrm{H}, 0.9852743167,0.1484129466,-5.4718044549$

$\mathrm{H}, 2.3160865276,-1.0183195702,-5.4533854226$

C,0.6342481555,-1.5041334614,-2.9163149652

$\mathrm{H}, 0.9488930581,-2.3786593213,-2.3475648576$

$\mathrm{H},-0.3576087422,-1.2020748067,-2.5738391833$

$\mathrm{H}, 0.6002441603,-1.751698979,-3.9761296202$

C,3.4825365705,1.8512295718,-0.7096531412

$\mathrm{H}, 2.7633674501,2.0008967321,0.0961770832$

$\mathrm{H}, 4.3766019888,1.3660402435,-0.309867817$

$\mathrm{H}, 3.7562474783,2.8198317995,-1.1273744547$

C,-0.8070279697,-3.6800950896,-0.529659117

$\mathrm{H},-0.9851599955,-3.4379911666,-1.5831944412$ 
$\mathrm{H}, 0.0614051337,-4.3437949419,-0.4532984327$

$\mathrm{H},-1.6764982944,-4.2474414409,-0.1792510665$

C,2.7172970078,-0.6322847414,1.533553096

$\mathrm{H}, 2.2490721771,-0.9780228087,2.4585322537$

$\mathrm{H}, 3.4724563923,-1.3639195896,1.2368774542$

$\mathrm{H}, 3.1962303507,0.3298258866,1.7252874881$

C,-1.0287457111,1.5231543038,2.2889097426

$\mathrm{H},-1.1249625362,0.6977469269,2.9985599805$

$\mathrm{H},-0.2353681938,2.1924055349,2.6417284687$

$\mathrm{H},-1.9665887944,2.0902783331,2.2840572671$

C,-0.6722030912,2.3889412287,-0.6341138033

$\mathrm{H},-0.5467502889,2.1008475069,-1.6822201531$

$\mathrm{H},-1.6255495003,2.919233627,-0.5494473013$

$\mathrm{H}, 0.1286790917,3.091507352,-0.3732663028$

C,-1.6170243407,-0.6777104763,-0.1314706017

$\mathrm{N},-2.6166192223,-0.7671869015,-0.9623979129$

C,-3.4609348596,0.3154386952,-1.2248637708

C,-4.0143921465,1.1147143979,-0.2034251169

C,-3.8493495139,0.5758522393,-2.5541033426

C,-4.8883101429,2.1584464404,-0.5093885891

$\mathrm{H},-3.7817955108,0.8771968262,0.8302266294$

C,-4.7096568067,1.6273313563,-2.8536010002

$\mathrm{H},-3.4525567519,-0.0626331827,-3.3382775466$

C,-5.2336776991,2.4301211061,-1.8343669269

$\mathrm{H},-5.3111400163,2.7554236219,0.2950041659$

$\mathrm{H},-4.9830329748,1.8189890542,-3.8882955362$

$\mathrm{H},-5.916188746,3.2414089514,-2.0698209646$
Zero-point correction $=$
Thermal correction to Energy=
Thermal correction to Enthalpy=
0.437478 (Hartree/Particle)
0.468437
0.469381
Thermal correction to Gibbs Free Energy=
Sum of electronic and zero-point Energies=
0.375034
$-3521.238822$
$-3521.207864$
$-3521.206919$
Sum of electronic and thermal Enthalpies=
$-3521.301266$
Sum of electronic and thermal Free Energies=

Table S61. Cartesian coordinates and energy values for the 36-Ph.

Ge,-0.034010074,-2.6885094033,-0.1086903539

$\mathrm{Si},-0.5703237816,-0.6639182132,1.0957952274$

$\mathrm{Si}, 1.2946832972,-0.9214511612,-1.1609554605$

$\mathrm{N},-3.1273170459,-0.2120731416,-0.4131510905$

$\mathrm{N},-2.409395484,1.5921543783,0.5310544795$

C,-2.1310809977,0.2731019045,0.3711650897

C,-4.0264434716,0.7981439686,-0.7480276742

C,-3.5741137654,1.9393555161,-0.1432242921

C,-5.2232114115,0.5812893947,-1.614370809

$\mathrm{H},-5.7653434505,1.519973135,-1.7419406774$

$\mathrm{H},-5.9199824382,-0.1442288763,-1.1800237033$

$\mathrm{H},-4.9457430441,0.2231839709,-2.6115372328$

C,-4.1283162157,3.3255924502,-0.1426214629

$\mathrm{H},-5.0662463105,3.3572665259,-0.6996500849$

$\mathrm{H},-3.4421369853,4.0400609799,-0.6115207366$

$\mathrm{H},-4.3333910897,3.6810939846,0.8730265872$

C,-1.6059290026,2.5263072187,1.3232155108

$\mathrm{H},-0.5762833768,2.1705724114,1.387750548$

$\mathrm{H},-1.6075572194,3.5000939748,0.8315321384$

$\mathrm{H},-2.0285960415,2.6320254323,2.3263639519$

C,-3.2456038175,-1.6051225565,-0.8656824533 
$\mathrm{H},-2.432753779,-1.8600779467,-1.5486658428$

$\mathrm{H},-3.1724710792,-2.2839806596,-0.0174342473$

$\mathrm{H},-4.2095589222,-1.7276282579,-1.357168673$

C, $-0.7024652255,-0.3179348134,2.970361403$

$\mathrm{H},-0.2181439042,0.6305364414,3.2232321957$

$\mathrm{H},-1.7377348633,-0.3000520857,3.3276017665$

$\mathrm{H},-0.1813215966,-1.1099009052,3.5169695626$

C,1.2779610313,-3.5096429386,1.215289942

$\mathrm{H}, 1.9312558187,-2.8259349896,1.7628993756$

$\mathrm{H}, 0.699511909,-4.1006408371,1.930372232$

$\mathrm{H}, 1.9011003695,-4.2024072059,0.6421907943$

C,3.0566203624,-1.1437427697,-1.8526598221

$\mathrm{H}, 3.7290491436,-1.630091295,-1.1410101698$

$\mathrm{H}, 3.0043077168,-1.776977897,-2.7467116768$

$\mathrm{H}, 3.5003230094,-0.1853967487,-2.1407113868$

C,0.2839643704,-0.1915122158,-2.6159033981

$\mathrm{H},-0.7346958326,0.0858900143,-2.3248456456$

$\mathrm{H}, 0.7694682911,0.7026081176,-3.0242752729$

$\mathrm{H}, 0.2072397157,-0.9315560085,-3.4209340392$

C,0.9329564873,0.289657286,0.3207654977

$\mathrm{N}, 1.5145267464,1.2156233791,1.0132841467$

C,2.8119194825,1.688942732,0.7425351111

C,3.9306409108,0.8396615927,0.7383710549

C,3.0115879457,3.0705951034,0.5746055846

C,5.209996658,1.3583797294,0.5403475141

$\mathrm{H}, 3.7852346383,-0.2205469221,0.9155766751$

C,4.289091801,3.5786379713,0.356494766

$\mathrm{H}, 2.1492576237,3.7301259946,0.6060594134$

C,5.3973571604,2.7265904108,0.3392200622

$H, 6.064724281,0.6872815608,0.5444169225$

$\mathrm{H}, 4.4229816953,4.6469674653,0.2084780674$

H,6.394496761,3.1270738038,0.1828895854

Zero-point correction=

Thermal correction to Energy=

Thermal correction to Enthalpy=

Thermal correction to Gibbs Free Energy=

Sum of electronic and zero-point Energies=

Sum of electronic and thermal Energies=

Sum of electronic and thermal Enthalpies=

Sum of electronic and thermal Free Energies=
0.438094 (Hartree/Particle)

0.468717

0.469661

\subsection{9}

$-3521.243065$

$-3521.212442$

$-3521.211497$

$-3521.304940$

Table S62. Cartesian coordinates and energy values for the TS1-Ph.

Ge,-0.2839189295,1.2010296453,-0.8257440147

$\mathrm{Si},-2.701348291,-1.2498471138,1.017018267$

$\mathrm{Si},-1.5409227497,0.691409336,1.177442744$

$\mathrm{N}, 2.5095992334,0.7435766697,0.5268692242$

$\mathrm{N}, 1.9566272849,-0.814108698,-0.8482594852$

C,-3.7611657621,-1.5585527333,-1.1267147708

C,-4.3483213073,-2.6489036654,-0.9819198618

C, $1.5042242395,0.3436458234,-0.2945562884$

C,3.5877454334,-0.1428395146, 0.4833892404

C,3.2314879959,-1.1351605178,-0.384197714

$\mathrm{C}, 4.8382398398,0.052014767,1.2748854076$

C,3.9647353154,-2.357697268,-0.8255153832

$\mathrm{H}, 4.6527380681,0.0173268592,2.3549202156$

$\mathrm{H}, 5.3160567726,1.0127877175,1.0523588089$

$\mathrm{H}, 5.5579567948,-0.7354628932,1.0422440106$

$\mathrm{H}, 4.9267912772,-2.4281914814,-0.314350673$ 
$\mathrm{H}, 4.1625626675,-2.3511882118,-1.9038198259$ $\mathrm{H}, 3.4021436736,-3.2710063464,-0.6008840266$ C,-1.6142138665,-2.8162494859,1.0516295235 $\mathrm{H},-0.7060743441,-2.6678234492,0.4589052838$ $\mathrm{H},-1.3098377358,-3.0540003262,2.0760266776$ $\mathrm{H},-2.1482476515,-3.681398713,0.6451320357$ C,2.4171737599,1.9084907034,1.3980999505 $\mathrm{H}, 2.7055433372,2.821666087,0.8715405212$ $\mathrm{H}, 1.3834823723,1.9983013092,1.7341622134$ $\mathrm{H}, 3.0700545467,1.7692398079,2.26028611$ C,1.1941437852,-1.6316168302,-1.7851752983 $\mathrm{H}, 0.8418701885,-2.547198649,-1.3016783348$ $\mathrm{H}, 0.3354031089,-1.0398004745,-2.1158799752$ $\mathrm{H}, 1.8169143506,-1.8936060778,-2.6442967343$ C,-4.1255984099,-1.5040680562,2.2597794189 $\mathrm{H},-4.8300108682,-2.2617436163,1.8993752199$ $\mathrm{H},-3.7397863175,-1.8389746046,3.2282179655$ $\mathrm{H},-4.6843732341,-0.5773670903,2.4211031648$ C,0.1552669902,3.1665725361,-0.6451115121 $\mathrm{H}, 1.0728511401,3.3866034378,-1.200674943$ $\mathrm{H},-0.6598498041,3.6606124157,-1.1826663997$ $\mathrm{H}, 0.2234511286,3.6012718382,0.3551820921$ C,-2.7673584878,2.1756735246,1.3791026846 $\mathrm{H},-2.9970774051,2.6322488004,0.4105989806$ $\mathrm{H},-3.704843427,1.885519098,1.864300388$ $\mathrm{H},-2.3016014305,2.9496369122,2.0002849599$ $\mathrm{H},-4.6839686376,-3.5179848547,-0.45663487$ C,-3.3999061186,-0.5053223613,-2.0728032978 C,-3.8011237437,0.8243744846,-1.8766698951 C,-2.6586886554,-0.8289783752,-3.2194395601 C,-3.4724695727,1.8052198087,-2.809282906 $\mathrm{H},-4.365781138,1.0815942015,-0.9872752993$ C,-2.3330400174,0.1573182101,-4.1515813557 $\mathrm{H},-2.3560941186,-1.8594003413,-3.3778649305$ C,-2.7404322019,1.4749347018,-3.9530439315 $\mathrm{H},-3.7969753779,2.8291274542,-2.6472743123$ $\mathrm{H},-1.7579865831,-0.1079094371,-5.0343034413$ $\mathrm{H},-2.4822704479,2.2424425275,-4.6765876776$
Zero-point correction=
Thermal correction to Energy=
Thermal correction to Enthalpy=
0.444182 (Hartree/Particle) 0.476449 0.477393
Thermal correction to Gibbs Free Energy= Sum of electronic and zero-point Energies= Sum of electronic and thermal Energies= Sum of electronic and thermal Enthalpies= Sum of electronic and thermal Free Energies= 0.379707 $-3505.085302$ $-3505.053035$ $-3505.052091$ $-3505.149778$

Table S63. Cartesian coordinates and energy values for the TS2-Ph.

Ge,-0.7123257253,-1.2762895245,-0.0760503449

$\mathrm{Si}, 2.0387343101,-0.479354228,2.8402070615$

Si,0.6164619582,-2.0102408674,1.76606475

$\mathrm{N},-3.1067882199,-0.2684412629,1.6396684102$

$\mathrm{N},-1.8368452928,1.3156416368,0.9201762438$

C,3.1465988754,0.8097253826,1.7118657249

C,3.8657669152,1.2879925892,2.6443952252

C,-1.9767999238,-0.037233196,0.9216255882

C,-3.6782981699,0.9260340354,2.0818715401

C,-2.8663179768,1.9286824531,1.6333167661 
C, $-4.9328902288,0.9808409596,2.8887824581$

C,-2.9578609594,3.4079963594,1.807748678

$\mathrm{H},-4.8184744209,0.490863255,3.8629482638$

$\mathrm{H},-5.7715553336,0.4999724712,2.372811451$

$\mathrm{H},-5.2137693417,2.018875644,3.0773198764$

$\mathrm{H},-3.8152152479,3.6630800299,2.4336242491$

$\mathrm{H},-3.0811582925,3.9283587672,0.8508096648$

$\mathrm{H},-2.0621059883,3.8143392367,2.2904722778$

C,0.9603354869,0.6169471817,3.9650430427

$\mathrm{H}, 0.1265531124,1.0688853948,3.4159899788$

$\mathrm{H}, 0.5328799125,0.0161809878,4.7742892166$

$\mathrm{H}, 1.5550289673,1.4204390735,4.4118267851$

C,-3.5839064118,-1.6043309909,1.9746007642

$\mathrm{H},-4.1915507343,-2.0224374948,1.1679949208$

$\mathrm{H},-2.7153605812,-2.2432176939,2.139581413$

$\mathrm{H},-4.1805586366,-1.5584773637,2.8858692988$

C,-0.7252235547,2.0265013627,0.294057262

$\mathrm{H}, 0.0515027749,2.2591844111,1.026735079$

$\mathrm{H},-0.3002599348,1.3793658504,-0.4772158196$

$\mathrm{H},-1.088189012,2.951523086,-0.157507032$

C,3.2499954281,-1.4810497937,3.9149679744

$\mathrm{H}, 3.6463141519,-0.8878034117,4.7441298791$

$\mathrm{H}, 2.7274707231,-2.3486254269,4.3341962448$

$\mathrm{H}, 4.0969095559,-1.8459492731,3.3256299543$

C,-1.8914055492,-2.8349898539,-0.5781133671

$\mathrm{H},-2.7546080835,-2.4628415949,-1.1388129341$

$\mathrm{H},-1.2910589122,-3.4206909259,-1.2806778978$

$\mathrm{H},-2.2352687857,-3.5002172417,0.2172190419$

C, $1.6276658184,-3.4808540776,1.0110270973$

$\mathrm{H}, 1.7607769048,-3.3869010886,-0.0725823548$

$\mathrm{H}, 2.6118447673,-3.5924823615,1.4761444575$

H,1.0772053549,-4.4135550964,1.1858249903

$\mathrm{H}, 4.5851364415,1.8044312616,3.2426630787$

C,2.957212179,1.0935613272,0.2676456077

C,2.5708734289,0.1059243403,-0.6424247019

C,3.1762552278,2.4007477017,-0.2059045073

C,2.3970529887,0.4082106855,-1.9941436812

$\mathrm{H}, 2.3967178734,-0.9018936958,-0.2873378867$

C,3.0030295027,2.7038334056,-1.5538474641

$\mathrm{H}, 3.4839805269,3.1669797361,0.4998872158$

C,2.6105377183,1.7074059004,-2.4543512099

$\mathrm{H}, 2.0776691452,-0.3727061487,-2.6770153813$

$\mathrm{H}, 3.1746032554,3.7180616178,-1.9047802455$

$\mathrm{H}, 2.4697113126,1.9460182384,-3.5048257138$

Zero-point correction=

Thermal correction to Energy=

Thermal correction to Enthalpy=

Thermal correction to Gibbs Free Energy=

Sum of electronic and zero-point Energies=

Sum of electronic and thermal Energies=

Sum of electronic and thermal Enthalpies=

Sum of electronic and thermal Free Energies=
0.443749 (Hartree/Particle)

0.476164

0.477108

0.378289

$-3505.084347$

$-3505.051933$

$-3505.050989$

$-3505.149808$

Table S64. Cartesian coordinates and energy values for the TS3-Ph.

Ge,-0.0049576035,-2.0223727592,0.1107160785

Si,-1.8171987417,1.9334790216,-0.3412120816

$\mathrm{Si},-1.8224459923,-0.4263809999,-0.1353850613$

$\mathrm{N}, 1.3510519683,-1.5029571568,-2.6674532124$ 
$\mathrm{N}, 2.2316168196,-0.4084473513,-1.037052453$ C,-2.4491493197, $0.2745633253,1.5598679067$ C,-2.4408057323,1.6304326414,1.4096378804 C,1.2034478669,-1.250382923,-1.3396277345 C,2.4661269732,-0.8436907761,-3.192244887 C,3.0164011651,-0.1425605733,-2.158996474 C,2.8753140639,-0.9527001188,-4.6233410039 C,4.2061498027, $0.7567551553,-2.11258423$

$\mathrm{H}, 2.1166311659,-0.5457766054,-5.3025951228$ $\mathrm{H}, 3.0569348703,-1.9926829104,-4.917504966$ $\mathrm{H}, 3.7988554706,-0.3960250236,-4.7944591434$ $\mathrm{H}, 4.6500477926,0.8505252781,-3.1055953162$ $\mathrm{H}, 4.9820943466,0.3765376134,-1.4379832804$ $\mathrm{H}, 3.939549167,1.763560935,-1.7714594281$ C,- $0.2219619738,2.9705379359,-0.4435799077$ $\mathrm{H}, 0.4974259176,2.6701597978,0.3242591271$ $\mathrm{H}, 0.257206605,2.8682994231,-1.4239142985$ $\mathrm{H},-0.4398906553,4.0344552215,-0.2922104765$ C, $0.4172500059,-2.303786128,-3.4463097424$ $\mathrm{H}, 0.7434486314,-3.3457102591,-3.5146238213$ $\mathrm{H},-0.5508567571,-2.2672467649,-2.9517777344$ $\mathrm{H}, 0.3293369586,-1.8907257879,-4.4526484755$ C,2.4684665908,0.1608278001,0.2847341171 $\mathrm{H}, 2.1957541989,1.2187359721,0.3017568798$ $\mathrm{H}, 1.8505611231,-0.3873177118,1.0030509757$ $\mathrm{H}, 3.5224367744,0.0534500826,0.5525701487$ C,-3.0743137636,2.7458582085,-1.5175281561 $\mathrm{H},-3.2736859534,3.7848656929,-1.2292365571$ $\mathrm{H},-2.6985434335,2.7557317946,-2.5472862605$ $\mathrm{H},-4.0271086746,2.2079869516,-1.5150720547$ C, $-0.80216251,-3.6789274706,-0.7255976667$ $\mathrm{H},-0.0065880743,-4.3569033579,-1.0508258678$ $\mathrm{H},-1.2888195963,-4.1610080872,0.1285555725$ $\mathrm{H},-1.5431208745,-3.5761083201,-1.5222400331$ C,-3.3385548823,-1.1857102958,-1.0390401605 $\mathrm{H},-3.5929722104,-2.171529145,-0.6357394039$ $\mathrm{H},-4.2152118412,-0.5388270249,-0.9290293562$ $\mathrm{H},-3.1414738135,-1.3078630503,-2.1106538483$ $\mathrm{H},-2.7458057512,2.3105363602,2.2108279924$ C,-2.9045995366, $-0.4264600419,2.7828240166$ C,-2.2476421395,-1.5764065392,3.2608793552 C,-4.0222136111, $0.0404061965,3.5004388751$ C,-2.6819130109,-2.2169617735,4.4208600863 $\mathrm{H},-1.3713163692,-1.9531311833,2.7394211468$ C,-4.4614964717,-0.6073097582,4.653297378 $\mathrm{H},-4.5574054334,0.9105447914,3.1314631855$ C,-3.7909683434,-1.7392272528,5.1211758673 $\mathrm{H},-2.1508084621,-3.0952426952,4.7779730106$ $\mathrm{H},-5.3318885591,-0.23135491,5.1845906659$ $\mathrm{H},-4.1325522871,-2.2462567032,6.0192607299$

Zero-point correction= Thermal correction to Energy= Thermal correction to Enthalpy= Thermal correction to Gibbs Free Energy= Sum of electronic and zero-point Energies= Sum of electronic and thermal Energies= Sum of electronic and thermal Enthalpies= Sum of electronic and thermal Free Energies=
0.448698 (Hartree/Particle) 0.478962 0.479906 0.387494 $-3505.197546$ $-3505.167282$ $-3505.166338$ $-3505.258750$ 
Table S65. Cartesian coordinates and energy values for the TS4-Ph.

Ge, $0.1012982277,0.8771832047,-0.6039193642$

Si, $2.4169368598,2.0146194484,0.3537954455$

Si, $1.6712672713,-0.5332834975,0.4608242528$

$\mathrm{N},-1.9617249713,0.1432842291,1.5560333811$

$\mathrm{N},-1.4313675615,2.2181158822,1.7135313493$

C,3.2227705859,-0.3519519021,-0.6470109341

C,3.589648426, $0.958053377,-0.6614998922$

C,-1.1656139283,1.0945779049,1.0020987205

C,-2.7055331279,0.6566904572,2.6169178325

C,-2.3633349588, 1.9740748975,2.7226072898

C,-3.659523286,-0.1720984233,3.4104150592

C, $-2.8212881063,3.0267110356,3.6761665425$

$\mathrm{H},-3.1550676248,-1.0002625291,3.9213006946$

$\mathrm{H},-4.445289284,-0.6036075206,2.7799957073$

$\mathrm{H},-4.1468535134,0.4378618418,4.1733824112$

$\mathrm{H},-3.5043611734,2.597441709,4.4116020387$

$\mathrm{H},-3.3530402998,3.8400303184,3.1686760805$

$\mathrm{H},-1.9824287291,3.4711472928,4.2230355813$

C, $2.3231440477,3.7403477345,-0.4795190448$

$\mathrm{H}, 1.9742258457,3.6500817986,-1.5123737759$

$\mathrm{H}, 1.6500415404,4.4301126498,0.0424714779$

$\mathrm{H}, 3.3166761812,4.2093481352,-0.4829258029$

C,-2.0675997526,-1.2335593473,1.0870903484

$\mathrm{H},-3.0245272052,-1.3918640247,0.5814588083$

$\mathrm{H},-1.2516389547,-1.4245776584,0.3923615096$

$\mathrm{H},-1.9872924503,-1.9213582583,1.9313352885$

C,- $-0.8477885194,3.5289681836,1.4487337052$

$\mathrm{H},-0.0223531716,3.7358449105,2.1347692469$

$\mathrm{H},-0.4715838787,3.5351801992,0.426707477$

$\mathrm{H},-1.6141367995,4.2981779098,1.5627779685$

C,3.0221987947,2.4150473033,2.1275000299

$\mathrm{H}, 3.7266940414,3.2565463827,2.1408395797$

$\mathrm{H}, 2.1850191877,2.6732591817,2.7875268421$

$\mathrm{H}, 3.506456786,1.5333312514,2.5565152783$

C,-1.3063498232,0.4957981987,-2.0101536104

$\mathrm{H},-1.9788235482,1.3517567913,-2.1280028827$

$\mathrm{H},-0.7667055372,0.3614874682,-2.9519808606$

$\mathrm{H},-1.8998434184,-0.4057708151,-1.8232080147$

C, $1.5136791924,-2.3650879549,1.0547087767$

$\mathrm{H}, 1.2798338298,-3.0572381537,0.2374212541$

$\mathrm{H}, 2.4697884872,-2.6817296757,1.4868877073$

$\mathrm{H}, 0.748541125,-2.4799318839,1.829777822$

$\mathrm{H}, 4.3442259826,1.3298622298,-1.3619956177$

C,3.9321482873,-1.408231344,-1.4004378223

C,3.2246987416,-2.417754902,-2.0778742722

C,5.338031034,-1.4356802567,-1.4623565592

C,3.8922986787,-3.4008706091,-2.806028942

$\mathrm{H}, 2.1386888447,-2.407654341,-2.0470670039$

C,6.0072789164,-2.4240727862,-2.1815942706

$\mathrm{H}, 5.8998492318,-0.6775616949,-0.9245248561$

C,5.2878136076,-3.4110073132,-2.8590176988

$\mathrm{H}, 3.32281581,-4.1625039631,-3.3321533849$

$\mathrm{H}, 7.0937808316,-2.4273005025,-2.2104056271$

$\mathrm{H}, 5.8093690475,-4.1832079397,-3.41746627$

Zero-point correction=

Thermal correction to Energy=

Thermal correction to Enthalpy=

Thermal correction to Gibbs Free Energy=
0.447053 (Hartree/Particle)

0.477793

0.478737

0.384590 
Sum of electronic and zero-point Energies= Sum of electronic and thermal Energies= Sum of electronic and thermal Enthalpies= Sum of electronic and thermal Free Energies=
$-3505.151172$

$-3505.120432$

$-3505.119488$

$-3505.213635$

Table S66. Cartesian coordinates and energy values for the TS5-Ph.

$\mathrm{Ge},-2.534082511,-1.140426991,-0.9406589679$

Si,-2.4827417595,-0.2807230265,1.3411435322

Si,-0.8760823544,0.390015569,-1.2918955295

$\mathrm{N}, 2.5580335638,-0.824621207,-0.2863750747$

$\mathrm{N}, 1.1796103053,-2.4487590519,-0.3404466541$

C,-1.2314099059,1.1382022711,1.3566327934

$\mathrm{H},-1.1234077447,1.757705524,2.253821576$

C,- $-0.4951112442,1.4582752465,0.2648963216$

C,1.2938168515,-1.1331021596,-0.6932949652

C,3.2141192462,-1.9028127691,0.3159156237

C, $2.3324824281,-2.9419669275,0.2838586601$

C,4.6057801962,-1.8139108172,0.8488318634

C,2.4715213709,-4.3439247295,0.779216413

$\mathrm{H}, 4.697343962,-1.0487844608,1.6289121172$

$\mathrm{H}, 5.3312886238,-1.5668640023,0.0643757762$

$\mathrm{H}, 4.9089110495,-2.7676460547,1.2865294862$

$\mathrm{H}, 3.4384982342,-4.4749974798,1.2700102481$

$\mathrm{H}, 2.4161165934,-5.079218935,-0.0328652992$

$\mathrm{H}, 1.694913717,-4.6013477864,1.5086393813$

C,-4.1458015351,0.3500960756,2.0199752305

$\mathrm{H},-4.5630336442,1.1470071051,1.3965489858$

$\mathrm{H},-4.8801933222,-0.4610442382,2.0652536495$

$\mathrm{H},-4.0228830487,0.7480811999,3.0346511168$

C,3.1851323675,0.4714384711,-0.4981274106

$\mathrm{H}, 4.0776606424,0.3681564978,-1.1239174544$

$\mathrm{H}, 2.4709030286,1.123663821,-0.993020012$

$\mathrm{H}, 3.4684026154,0.9317613991,0.4521188892$

C,0.0008924069,-3.2633771994,-0.6145766354

$\mathrm{H},-0.7687873924,-3.1238963732,0.1518795694$

$\mathrm{H},-0.4195068428,-2.9925088815,-1.5821526989$

$\mathrm{H}, 0.2818717766,-4.3173132871,-0.6364334561$

C,-1.8800016523,-1.6424775062,2.5373072605

$\mathrm{H},-1.8596278599,-1.2599515768,3.5647845347$

$H,-2.5483827795,-2.5105414424,2.5231671564$

$\mathrm{H},-0.8684369115,-1.9824070446,2.2935414118$

C,-2.758559233,1.192945615,-1.80104272

$\mathrm{H},-3.7392607666,0.8025315995,-1.4806527128$

$\mathrm{H},-2.6981391039,2.1975636621,-1.3730179639$

$\mathrm{H},-2.8007956859,1.2293274642,-2.8915212499$

C,0.0109129631,0.8138835104,-2.9179521633

$\mathrm{H},-0.368349438,1.7357110523,-3.3698019862$

$\mathrm{H}, 1.0903533245,0.9014076444,-2.7882940376$

$\mathrm{H},-0.1693288035,0.0007122261,-3.6294491896$

$\mathrm{C}, 0.487669205,2.5728349937,0.3073575857$

C, $0.6815368136,3.4269503618,-0.7938043347$

C, $1.2557763239,2.8189683256,1.4632444815$

C, $1.5834995056,4.49000636,-0.7364340654$

$\mathrm{H}, 0.1037771547,3.2697093894,-1.6981017112$

C,2.1617236097,3.8757782055,1.5201980252

$\mathrm{H}, 1.1443024839,2.1575968167,2.3170788713$

C,2.3304882941,4.7195537237,0.4194304373

$\mathrm{H}, 1.7010376664,5.1410299297,-1.5983996723$

$\mathrm{H}, 2.7412211429,4.0395793614,2.4249367362$ 


\section{$H, 3.0372680724,5.5431225259,0.4620472306$}

Zero-point correction $=$

Thermal correction to Energy=

Thermal correction to Enthalpy=

Thermal correction to Gibbs Free Energy=

Sum of electronic and zero-point Energies= Sum of electronic and thermal Energies=

Sum of electronic and thermal Enthalpies=

Sum of electronic and thermal Free Energies=
0.448208 (Hartree/Particle)

0.478395

0.479339

0.387285

$-3505.157859$

$-3505.127673$

$-3505.126728$

$-3505.218783$

Table S67. Cartesian coordinates and energy values for the TS9-Ph.

$\mathrm{Ge}, 0.81940492,-1.0701059521,-2.3284583341$

Si,1.9297987423,-0.5752055404,1.284585327

Si, $1.6306897083,-2.1316365187,-0.2857632304$

$\mathrm{N},-2.0954892806,-1.1636318433,-1.1856366175$

$\mathrm{N},-1.3721666573,0.8419649675,-1.4710670805$

C,3.2400019155,2.4535451459,0.4881235733

C, $2.8681373387,1.5883426903,-0.2923721752$

C, $-1.0078147159,-0.4619753316,-1.5968691906$

C,-3.1347316426,-0.3141638436,-0.8069141349

C,-2.6714861461, 0.9595129324,-0.9792299162

C,- $-4.4546937131,-0.8098464642,-0.3169507082$

C,-3.3263795548,2.2768515094,-0.7272321401

$\mathrm{H},-4.3594113143,-1.3752801434,0.6173657048$

$\mathrm{H},-4.9423756886,-1.4621857198,-1.0498515647$

$\mathrm{H},-5.1254173569,0.0298503467,-0.1245512693$

$\mathrm{H},-4.3238127132,2.1286818376,-0.3089376453$

$\mathrm{H},-3.4378195531,2.8635582274,-1.6465581032$

$\mathrm{H},-2.7554798867,2.8839493749,-0.0158477668$

C, $0.4227641995,0.3399528087,2.0056912297$

$\mathrm{H},-0.400464027,0.3764189629,1.2861927415$

$\mathrm{H}, 0.0578541169,-0.1494040679,2.9155041176$

$\mathrm{H}, 0.6961011631,1.3683181049,2.2675712742$

C,-2.1439337265,-2.6201453927,-1.1165335114

$\mathrm{H},-2.4965136878,-3.0446456323,-2.0602898316$

$\mathrm{H},-1.136223832,-2.9805346883,-0.9020677797$

$\mathrm{H},-2.8150605915,-2.9226739481,-0.3118939633$

C,- $-0.5011672757,1.9774604333,-1.7622684892$

$\mathrm{H},-0.0262719289,2.3475998013,-0.8498692327$

$\mathrm{H}, 0.2763301015,1.6354798784,-2.4495613161$

$\mathrm{H},-1.0831230243,2.7768791749,-2.2245133897$

C,3.2468689517,-0.7067028187,2.650093101

$\mathrm{H}, 3.639399522,0.2893876031,2.8852781163$

$\mathrm{H}, 2.8283107953,-1.1255456546,3.5718673717$

$\mathrm{H}, 4.0852484083,-1.3348976519,2.336527851$

C, $0.2207502719,-2.5925379464,-3.5156339216$

$\mathrm{H},-0.4619180158,-2.1952545524,-4.274527865$

$\mathrm{H}, 1.1206805437,-2.9212819624,-4.0451156627$

$\mathrm{H},-0.2352085852,-3.467683875,-3.0461330545$

C,3.3347178009,-2.915904753,-0.7604934028

$\mathrm{H}, 3.1853681976,-3.9457482596,-1.1034907437$

$\mathrm{H}, 3.8069687625,-2.3596883996,-1.5773028555$

$\mathrm{H}, 4.0279867173,-2.9490244955,0.0856083756$

$\mathrm{H}, 2.6277521974,1.0517188967,-1.192723624$

C,3.6688275775,3.3818544602,1.4762867083

C,2.7875816819,4.3617927945,1.9821188435

C,4.9928593227,3.3461744481,1.9658854723

C,3.2180004371,5.267661047,2.9463005777 
$H, 1.7695666148,4.3998679651,1.6077549528$

C,5.4126930821,4.2578106967,2.9292807721

$\mathrm{H}, 5.676796221,2.5977404657,1.5794674893$

C,4.5300747987,5.2213133984,3.4249297314

$\mathrm{H}, 2.5273081862,6.0149191327,3.3264589444$

H,6.4340920592,4.2164512335,3.2964098439

$\mathrm{H}, 4.8621025822,5.9304708873,4.1771195313$
Zero-point correction $=$
Thermal correction to Energy=
0.445123 (Hartree/Particle)
Thermal correction to Enthalpy=
0.477547
0.478492
Thermal correction to Gibbs Free Energy=
Sum of electronic and zero-point Energies=
Sum of electronic and thermal Energies=
0.377969
$-3505.100038$
$-3505.067613$
$-3505.066669$
Sum of electronic and thermal Free Energies=
$-3505.167192$

Table S68. Cartesian coordinates and energy values for the TS17-Ph.

Ge,-0.7121750312,-0.3144944043,-0.7510537753

Si,-2.2890881043,1.3235888121,0.3240086659

Si,-1.4247691671,-0.3574788392,1.6501857801

$\mathrm{N}, 2.3764538775,-0.8531513563,0.146919127$

$\mathrm{N}, 2.2757706263,1.0766187247,-0.7772113596$

C, $1.4902366487,0.0633799763,-0.3257930459$

C,3.6340747823,0.81583462,-0.5679945479

C,3.6976006309,-0.4144663242,0.0192120579

C,4.7135225099, 1.769741272,-0.9578837439

$\mathrm{H}, 4.579135919,2.7510241127,-0.488244369$

$\mathrm{H}, 4.7518649663,1.9280500452,-2.0423412503$

$\mathrm{H}, 5.6883562445,1.386656507,-0.6486562762$

C,4.8682669298,-1.2226190657,0.4706611473

$\mathrm{H}, 5.7950001731,-0.6643411374,0.3220989923$

$\mathrm{H}, 4.9580198134,-2.163155706,-0.0861670766$

$\mathrm{H}, 4.8020689808,-1.4769423698,1.5347592438$

C,-2.4351405616,-1.8120466457,2.351161822

$\mathrm{H},-2.9533163367,-1.5027931968,3.2660624567$

$\mathrm{H},-3.1760764157,-2.1683259487,1.6333252403$

$\mathrm{H},-1.7781727541,-2.6515663148,2.6068335363$

C, $-0.2216590963,0.2061689844,3.0286267546$

$\mathrm{H}, 0.3419320496,1.0996446066,2.7459936216$

$\mathrm{H},-0.7755644978,0.4306777821,3.9476912063$

$\mathrm{H}, 0.5021477138,-0.582939175,3.2661713536$

C, $-1.1909622852,2.8826786229,0.5714294189$

$\mathrm{H},-0.1577065997,2.6124547428,0.8076058608$

$\mathrm{H},-1.1795614949,3.4978321275,-0.3363214446$

$\mathrm{H},-1.5762101022,3.5047570917,1.3879466857$

C,-4.0344134739,2.0419815871, 0.027277104

$\mathrm{H},-4.2084722964,2.8639951093,0.7313887118$

$\mathrm{H},-4.1237920431,2.4466927592,-0.987227642$

$\mathrm{H},-4.8250729331,1.3023160443,0.1715033027$

C, $1.7537592541,2.2685564171,-1.4339995635$

$\mathrm{H}, 0.730948097,2.0617844298,-1.7524191202$

$\mathrm{H}, 1.752299218,3.1275957316,-0.7570998057$

$\mathrm{H}, 2.3605218404,2.5052130615,-2.3114239749$

C, $1.9841386617,-2.1398186239,0.7103901451$

$\mathrm{H}, 2.0231528213,-2.1190283875,1.8037330646$

$\mathrm{H}, 0.9656975746,-2.3615097323,0.3926134853$

$\mathrm{H}, 2.6502471526,-2.9248124533,0.344750097$

C,-2.428686025,-1.7879812035,-1.2197701916 
C,-3.3019516852,-0.9145577227,-0.9913931379

$\mathrm{H},-4.3139293302,-0.5619019331,-0.9930474713$

C,-2.1132825964,-3.1677311124,-1.5604184773

C,-3.1439263212,-4.1277532306,-1.5239961586

C,- $-0.8307250324,-3.5813818872,-1.9502194304$

C,-2.8923874898,-5.455647331,-1.8571832737

$\mathrm{H},-4.1390488654,-3.8137207794,-1.2249005597$

C,- $-0.5826983453,-4.9099107886,-2.2905696721$

$\mathrm{H},-0.0362781629,-2.8414226023,-1.9946160411$

C,-1.6097720788,-5.8539346478,-2.2430737948

$\mathrm{H},-3.6991184295,-6.182349052,-1.816762904$

$\mathrm{H}, 0.4161123229,-5.2094898451,-2.5964987623$

$\mathrm{H},-1.4137959433,-6.890296681,-2.5028422611$

Zero-point correction=

Thermal correction to Energy=

Thermal correction to Enthalpy=

Thermal correction to Gibbs Free Energy=

Sum of electronic and zero-point Energies=

Sum of electronic and thermal Energies=

Sum of electronic and thermal Enthalpies=

Sum of electronic and thermal Free Energies $=$
0.444816 (Hartree/Particle) 0.476782

0.477726

0.380443

$-3505.104024$

$-3505.072058$

$-3505.071114$

$-3505.168397$

Table S69. Cartesian coordinates and energy values for the TS19-Ph.

Ge,-1.0617908802,-0.774270361,-0.6622164685

Si, $1.0518670271,-0.9573589485,1.8200039836$

Si,-0.7989361975,-2.2956883985,1.1804086117

$\mathrm{N},-3.7106762148,0.4783963013,0.2135199931$

$\mathrm{N},-2.2424866353,2.0010908861,-0.1866506746$

C,3.0904868794,-0.0698982932,-0.1327343658

C, $1.893619395,-0.2893834392,0.1938110931$

C,-2.4318049916,0.6578498522,-0.1977843649

C,-4.3299123283,1.6996608793,0.4738485926

C,-3.396053089,2.6660706402,0.2251970067

C,-5.7441163768, 1.8127552563,0.9373612872

C,-3.470073043,4.1519986352,0.3388792394

$\mathrm{H},-5.8912588773,1.3470937578,1.9184590662$

$\mathrm{H},-6.440800755,1.3386475447,0.2373981195$

$\mathrm{H},-6.0280594794,2.8631035614,1.0261252465$

$\mathrm{H},-4.463665692,4.4573327169,0.6719796417$

$\mathrm{H},-3.2758738348,4.6451313976,-0.6204376383$

$\mathrm{H},-2.7442508393,4.5405966131,1.0621155676$

C, $0.3924805046,0.648147843,2.6187858444$

$\mathrm{H},-0.3458307923,1.100003426,1.9475498937$

$\mathrm{H},-0.1084589218,0.4413656764,3.5702754559$

$\mathrm{H}, 1.1897686309,1.3777527972,2.7974300608$

C,-4.3593030373,-0.8264042083,0.3392804492

$\mathrm{H},-4.8578577053,-1.0985875395,-0.5953578023$

$\mathrm{H},-3.5943011251,-1.566323417,0.5890403227$

$\mathrm{H},-5.0947711207,-0.788048628,1.1431447255$

C,-0.999301516,2.6786100978,-0.5479753899

$\mathrm{H},-0.5924383897,3.2072810078,0.3176158556$

$\mathrm{H},-0.2826056987,1.9239383401,-0.8750927038$

$\mathrm{H},-1.1825808406,3.3900123795,-1.3572524498$

C,2.390868002,-1.6865328952,2.9562043385

$\mathrm{H}, 3.1723237796,-0.9534946792,3.182895274$

$\mathrm{H}, 1.9443537493,-2.0113030096,3.9024798859$

$\mathrm{H}, 2.8694615249,-2.5571736758,2.497020262$

C,-1.8255006972,-1.4726309576,-2.3617431843 
$\mathrm{H},-2.0861279473,-0.6655266791,-3.0506356117$

$\mathrm{H},-1.0425587695,-2.0782324403,-2.824301242$

$\mathrm{H},-2.699569863,-2.1066013132,-2.1884420961$

C, $0.0337087749,-3.7325757018,0.1280407787$

$\mathrm{H},-0.7376509361,-4.280303433,-0.4251685002$

$\mathrm{H}, 0.7817371365,-3.3917728848,-0.5964659833$

$\mathrm{H}, 0.5214843957,-4.4496169734,0.7981537926$

$\mathrm{H}, 0.9048399338,-0.0992317382,-0.5308788267$

C,4.3972425989,0.1705610003,-0.5752131983

C,5.052371024,1.4002451365,-0.2971281066

C,5.1096213266,-0.8112055374,-1.3154432736

C,6.349149579,1.627067645,-0.7380612401

$\mathrm{H}, 4.5225495274,2.1595077782,0.2692518028$

C,6.4036436409,-0.5650001965,-1.7540735642

$\mathrm{H}, 4.623752436,-1.7567788529,-1.5328005643$

C, $7.0348568139,0.6512637461,-1.4701620418$

$\mathrm{H}, 6.8323687654,2.5738124461,-0.5121715714$

$\mathrm{H}, 6.9294028471,-1.3286305696,-2.3207416867$

$\mathrm{H}, 8.0483093821,0.8355411288,-1.8132214816$

Zero-point correction=

Thermal correction to Energy=

Thermal correction to Enthalpy=

Thermal correction to Gibbs Free Energy=

Sum of electronic and zero-point Energies=

Sum of electronic and thermal Energies=

Sum of electronic and thermal Enthalpies=

Sum of electronic and thermal Free Energies=
0.442500 (Hartree/Particle)

0.474021

0.474966

\subsection{6}

$-3505.118617$

$-3505.087096$

$-3505.086151$

$-3505.183531$

Table S70. Cartesian coordinates and energy values for the TS20-Ph.

Ge,-0.085953,0.080845,0.14029

$\mathrm{Si},-0.428672,2.453899,0.613669$

Si, $1.36863,3.292055,-0.336751$

$\mathrm{N},-2.883434,-1.263322,0.804749$

$\mathrm{N},-2.803264,-0.14943,-1.035771$

C,-2.058572,-0.474312,0.060018

C,-4.134601,-1.408983,0.197536

C,-4.081702,-0.703284,-0.968076

C, $-5.240179,-2.210505,0.800521$

$\mathrm{H},-6.134237,-2.147583,0.17701$

$\mathrm{H},-5.510566,-1.850971,1.800098$

$\mathrm{H},-4.978993,-3.271777,0.892076$

C,-5.116194,-0.506466,-2.025776

$\mathrm{H},-6.043484,-1.008065,-1.741778$

$\mathrm{H},-4.802915,-0.919224,-2.991954$

$\mathrm{H},-5.34768,0.553821,-2.178393$

C,-2.294024,0.676955,-2.123752

$\mathrm{H},-2.103536,1.692511,-1.764264$

$\mathrm{H},-1.343925,0.260635,-2.472429$

$\mathrm{H},-3.018282,0.692829,-2.937478$

C,-2.557733,-1.788926,2.122744

$\mathrm{H},-3.063167,-2.744316,2.273384$

$\mathrm{H},-1.485101,-1.940499,2.193823$

$\mathrm{H},-2.870901,-1.094174,2.909261$

C,- $0.707739,3.038554,2.432051$

$\mathrm{H},-0.484184,4.104938,2.540293$

$\mathrm{H},-1.756386,2.891473,2.712721$

$\mathrm{H},-0.089012,2.482462,3.142789$

C, $0.515021,-0.543644,1.965313$ 
$\mathrm{H}, 1.477262,-0.04391,2.106819$

$\mathrm{H},-0.130757,-0.270256,2.802822$

$\mathrm{H}, 0.703669,-1.622946,1.978423$

C, 1.79448,2.658682,-2.073715

$\mathrm{H}, 1.396926,1.642552,-2.162067$

$H, 1.364551,3.284255,-2.863284$

$\mathrm{H}, 2.879206,2.621899,-2.217438$

C,2.21061,4.96276,-0.008629

$\mathrm{H}, 1.861229,5.412594,0.924645$

H,3.293982,4.815208,0.07151

$\mathrm{H}, 2.030849,5.671252,-0.824878$

C,3.553432,-1.493857,-0.15753

C,2.664125,-2.090675,-1.061194

C,4.485908,-2.263888,0.549644

C,2.712748,-3.471335,-1.24137

$\mathrm{H}, 1.930722,-1.470038,-1.564274$

C,4.521982,-3.642512,0.353817

$\mathrm{H}, 5.164492,-1.775383,1.240351$

C,3.63893,-4.248585,-0.542277

$\mathrm{H}, 2.023838,-3.941112,-1.936883$

$\mathrm{H}, 5.24299,-4.24221,0.900814$

$\mathrm{H}, 3.670586,-5.323575,-0.692386$

C,3.560023,1.049731,0.179141

$\mathrm{N}, 3.531672,-0.119967,0.025841$

Zero-point correction=

Thermal correction to Energy=

Thermal correction to Enthalpy=

Thermal correction to Gibbs Free Energy=

Sum of electronic and zero-point Energies $=$

Sum of electronic and thermal Energies=

Sum of electronic and thermal Enthalpies=

Sum of electronic and thermal Free Energies=
0.435538 (Hartree/Particle)

0.467328

0.468272

0.370289

$-3521.180749$

$-3521.148959$

$-3521.148015$

$-3521.245998$

Table S71. Cartesian coordinates and energy values for the TS21-Ph.

Ge,-0.0325553453,-0.8455836266,-1.3548089085

Si, $0.1588608212,0.4328618103,0.7049801271$

Si, $1.8150824919,1.9576934079,0.1945366676$

$\mathrm{N},-2.9051044924,-2.0616754848,-0.8588455766$

$\mathrm{N},-2.8956257074,-0.0879500875,-1.7188890249$

C,-2.0678051726,-1.0513784783,-1.223051335

C,-4.2386634066,-1.7419216846,-1.1300503189

C,-4.2319006437,-0.4864827917,-1.6634175027

C,-5.3694028929,-2.6733776356,-0.8445640754

$\mathrm{H},-6.3114592823,-2.2387658938,-1.184794578$

$\mathrm{H},-5.473779916,-2.8824856321,0.2269741231$

$\mathrm{H},-5.2453472101,-3.6346572653,-1.3563515365$

C,-5.3600491128,0.3730354829,-2.1283993611

$\mathrm{H},-6.3119104846,-0.1383687312,-1.9716372785$

$\mathrm{H},-5.2861408018,0.6089560951,-3.1965423947$

$\mathrm{H},-5.4019115025,1.3224008736,-1.5823618711$

C,-2.4212967866,1.2091286614,-2.1832855463

$\mathrm{H},-2.161558733,1.8467616108,-1.33336204$

$\mathrm{H},-1.5200000201,1.0530524022,-2.7842005443$

$\mathrm{H},-3.1950302315,1.6876923662,-2.7837499022$

C,-2.4695037535,-3.2779486849,-0.1880874599

$\mathrm{H},-2.2990631667,-4.090855737,-0.9005608959$

$\mathrm{H},-1.5409582351,-3.0632107873,0.334813116$

$\mathrm{H},-3.2274511891,-3.5908722136,0.532350243$ 
C, $0.5963064187,-0.5496247804,2.3055433842$ $\mathrm{H}, 1.4305995244,-1.2380292889,2.1194176489$ $\mathrm{H}, 0.8637493533,0.1125311098,3.1362133653$ $\mathrm{H},-0.2729914915,-1.1355676667,2.6240731231$ C, $0.6590029764,-2.6895480493,-0.9239632868$ $\mathrm{H}, 1.7116317394,-2.6525413248,-1.2152265327$ $\mathrm{H}, 0.6187653901,-3.0177902187,0.1185349051$ $\mathrm{H}, 0.1813980409,-3.4363742776,-1.5669626942$ C, $1.6402142516,2.7156008302,-1.5243824459$ $\mathrm{H}, 1.3638122588,1.925817283,-2.2291285655$ $\mathrm{H}, 0.8487313326,3.4733238998,-1.5229191631$ $\mathrm{H}, 2.5715604817,3.1863320756,-1.8552701156$ C,2.5042049558,3.1444786811,1.4940548049 $\mathrm{H}, 2.7011646417,2.6245317874,2.4356479448$ $\mathrm{H}, 3.433784129,3.6147708549,1.1573790625$ $\mathrm{H}, 1.7682941867,3.9319120514,1.6930953379$ C,2.7037172044,0.3918942796,0.1298250554 $\mathrm{N}, 3.2487248528,-0.7103721184,0.2956346523$ C,4.6339661191,-0.9709714195,0.108296296 C, $5.4695665211,-0.1566861513,-0.6705639647$ C,5.1525777166,-2.1223373637,0.7120027365 C,6.8122751851,-0.4897391141,-0.8260786029 $\mathrm{H}, 5.04999948,0.7214672353,-1.1519519133$ C,6.5009820915,-2.4426058171, 0.5643148881 $\mathrm{H}, 4.4809374885,-2.7448543036,1.2945216648$ C,7.3343072925,-1.6292415804,-0.2062559261 $\mathrm{H}, 7.4558103269,0.1392170667,-1.4351538675$ $\mathrm{H}, 6.8995356811,-3.3323060587,1.0433023298$ $\mathrm{H}, 8.3822320042,-1.8853479977,-0.3319278574$
Zero-point correction=
Thermal correction to Energy=
Thermal correction to Enthalpy=
0.435566 (Hartree/Particle)
0.466676
0.467620
Thermal correction to Gibbs Free Energy=
Sum of electronic and zero-point Energies=
Sum of electronic and thermal Energies=
Sum of electronic and thermal Enthalpies=
0.371233
$-3521.185075$
$-3521.153964$
$-3521.153020$
Sum of electronic and thermal Free Energies $=$
$-3521.249407$

Table S72. Cartesian coordinates and energy values for the TS22-Ph.

Ge,-0.6238051816,1.0895182566,-0.4009538293

$\mathrm{Si}, 0.2995265989,-0.1930320915,1.2938968642$

Si, $1.9421846801,1.6284833856,0.6752815501$

$\mathrm{N},-3.2003588332,1.6758578823,1.1723037832$

$\mathrm{N},-1.9021086846,3.3903017259,1.1257793785$

C,-1.9925898812,2.1007302012,0.7145574544

C,-3.8590939373,2.6864723597, 1.8730148067

C,-3.0293358521,3.7708070015,1.8537468684

C,-5.2082920372,2.5044426899,2.4848588052

$\mathrm{H},-5.5497110561,3.441009326,2.9297584261$

$\mathrm{H},-5.2026013661,1.7472659808,3.2775868138$

$\mathrm{H},-5.9539172268,2.2001556992,1.741940234$

C,-3.189942046,5.1294900866,2.4502946957

$\mathrm{H},-4.1285903879,5.1889661085,3.0044612602$

$\mathrm{H},-3.2083823869,5.9156284147,1.6866059194$

$\mathrm{H},-2.3785815376,5.3649851802,3.1479571801$

C,- $0.7565116341,4.2547743606,0.8695164713$

$\mathrm{H},-0.0374607542,4.20206604,1.691424254$

$\mathrm{H},-0.273783165,3.9142396591,-0.0468652873$ 
$\mathrm{H},-1.0954029394,5.2850390004,0.7498839816$

C, $-3.7188242027,0.3255789155,0.9884972068$

$\mathrm{H},-4.3446080254,0.2622473242,0.0941741092$

$\mathrm{H},-2.8731947138,-0.3546033438,0.8841265869$

$\mathrm{H},-4.307313454,0.0394628046,1.8612831757$

C,- $0.1869013226,-1.5661182576,2.5391510186$

$\mathrm{H}, 0.6863744337,-1.7829628937,3.1633552341$

$\mathrm{H},-1.0177186891,-1.2752312431,3.1892231702$

$\mathrm{H},-0.4600049487,-2.487814996,2.0133081657$

C, $-1.7958574419,0.2803691039,-1.8186302568$

$\mathrm{H},-1.0978925544,-0.1540987907,-2.540018566$

$\mathrm{H},-2.4708342664,-0.5115928933,-1.4814413941$

$\mathrm{H},-2.3715428975,1.0532184178,-2.3380383713$

C,2.4110342365,2.8713743753,-0.7022079028

$\mathrm{H}, 1.9409924487,2.6033048194,-1.6506955035$

$\mathrm{H}, 2.1055261657,3.8938967629,-0.447326111$

$\mathrm{H}, 3.5022146674,2.8782627507,-0.818568816$

C,3.0294745569,2.1855281031,2.1503283555

$\mathrm{H}, 2.9892384654,1.449590714,2.9586695331$

$\mathrm{H}, 4.0755238585,2.3065641275,1.8411134594$

$\mathrm{H}, 2.6957601017,3.1476134675,2.5589402616$

C,2.0076044636,-0.2686952938,0.5559397294

$\mathrm{N}, 2.9309138572,-1.1725252967,0.6130837517$

C,4.227193638,-0.9122386083,0.1355388628

C,4.4837809222,-0.2053135142,-1.0555739097

C, $5.3171557305,-1.4533641619,0.840854165$

C,5.7910097651,-0.0312464949,-1.5079594101

$\mathrm{H}, 3.6463161104,0.172367511,-1.6333106224$

C,6.6214209441,-1.2589563405,0.3941183825

$\mathrm{H}, 5.1118351989,-2.018557239,1.7448788432$

C,6.867902176,-0.5469729932,-0.7834448205

$\mathrm{H}, 5.9693999356,0.5051526129,-2.4366714369$

$H, 7.4508126453,-1.6731525065,0.9616148635$

H,7.8849268338,-0.4089762002,-1.1389544741

Zero-point correction=

Thermal correction to Energy=

Thermal correction to Enthalpy=

Thermal correction to Gibbs Free Energy=

Sum of electronic and zero-point Energies=

Sum of electronic and thermal Energies=

Sum of electronic and thermal Enthalpies=

Sum of electronic and thermal Free Energies=
0.435829 (Hartree/Particle)

0.466403

0.467347

0.373341

$-3521.190477$

$-3521.159903$

$-3521.158958$

$-3521.252965$

Table S73. Cartesian coordinates and energy values for the TS23-Ph.

$\mathrm{Ge},-1.1103590103,-2.91782353,-0.876807841$

$\mathrm{Si},-0.4539580212,-1.8040175536,1.0592999282$

Si, $0.9260961402,-1.6407010478,-1.4568505839$

$\mathrm{N},-2.8850866025,0.6399328525,-0.3354050197$

$\mathrm{N},-1.7881002941,1.7085376371,1.1476090251$

C,-1.8349103716,0.4987628703,0.5253542017

C,-3.483391384,1.9044052467,-0.2519318627

C,-2.7823418067,2.5851574104,0.7001747534

C,-4.6471751714,2.3321163219,-1.0847004246

$\mathrm{H},-4.929506249,3.3575590679,-0.8359781536$

$\mathrm{H},-5.529377771,1.7020620926,-0.9197032963$

$\mathrm{H},-4.4200731112,2.3080414302,-2.1572057181$

C,-2.9470063957,3.9724836982,1.2271663067

$\mathrm{H},-3.8048610302,4.4594779134,0.7579572441$ 
$\mathrm{H},-2.0668300722,4.5957482809,1.0280856584$ $\mathrm{H},-3.1141433236,3.9816159883,2.3106417495$ C,- $0.8147789167,2.0363499356,2.1834023937$ $\mathrm{H}, 0.0222468338,1.3409073566,2.1064523892$ $\mathrm{H},-0.4391350612,3.0522922712,2.0345442281$ $\mathrm{H},-1.2664756286,1.9755761632,3.1796028535$ C,-3.3176236086,-0.4232130578,-1.2362794099 $\mathrm{H},-2.5239317821,-0.6743009606,-1.9480099325$ $\mathrm{H},-3.5805095375,-1.3220511136,-0.6742071883$ $\mathrm{H},-4.1888382361,-0.0948323386,-1.8030618463$ C, $-1.03571637,-1.6285936809,2.8571829082$ $\mathrm{H},-0.1758956365,-1.3856598647,3.4925199084$ $\mathrm{H},-1.7802412713,-0.8387037558,2.9665365887$ $\mathrm{H},-1.4650725198,-2.5679199134,3.2197482545$ C, $0.4736379814,-3.6157329354,1.0971735084$ $\mathrm{H}, 1.5585664775,-3.4979318378,1.1700204602$ $\mathrm{H}, 0.0920125259,-4.1122634603,1.9938802043$ $\mathrm{H}, 0.3104133752,-4.3501781417,0.2802260827$ C,2.3970405377,-2.6531537935,-2.1142080478 $\mathrm{H}, 2.7005177464,-3.441399133,-1.4187862438$ $\mathrm{H}, 2.1259777388,-3.1300605209,-3.0628703799$ $\mathrm{H}, 3.258790196,-2.0020406514,-2.2933822193$ C, $0.5901143535,-0.2276510743,-2.688883976$ $\mathrm{H},-0.2171024681,0.4261178658,-2.3452133593$ $\mathrm{H}, 1.490581766,0.3858159807,-2.8023076205$ $\mathrm{H}, 0.3238752276,-0.620706505,-3.6761723162$ C, $1.0400007065,-0.8678979639,0.3443479916$ $\mathrm{N}, 1.6810983375,0.1172401027,0.8609519366$ C,2.7916516338,0.7024341694,0.2155996368 C,3.9308668691,-0.0481230316,-0.119924991 C,2.8029596675,2.0886412509,-0.012795539 C, $5.0420472015,0.5720639309,-0.6928414522$ $\mathrm{H}, 3.9431890578,-1.1109109251,0.0987984276$ C,3.9073964962,2.697446154,-0.6031713071 $\mathrm{H}, 1.9304157368,2.6713565224,0.2658441938$ C,5.0344785199,1.9442134191,-0.9450645743 $\mathrm{H}, 5.9182806781,-0.0217206076,-0.9388749459$ $\mathrm{H}, 3.8931532773,3.7678799821,-0.7898076012$ $\mathrm{H}, 5.8995872891,2.4246985232,-1.3919666632$
Zero-point correction=
Thermal correction to Energy= Thermal correction to Enthalpy=
0.435808 (Hartree/Particle) 0.466168 0.467113
Thermal correction to Gibbs Free Energy= Sum of electronic and zero-point Energies= Sum of electronic and thermal Energies= Sum of electronic and thermal Enthalpies= 0.373769 $-3521.194242$ $-3521.163882$ $-3521.162937$ Sum of electronic and thermal Free Energies $=\quad-3521.256281$

Table S74. Cartesian coordinates and energy values for the Ac-Ph.
$\mathrm{C}, 0.6342533567,-0.000020921,0.00030864$
C, $-0.0800607895,-1.2130937608,0.0001561986$
C, $-0.0801148278,1.2130553343,0.0001514905$
C,-1.472119382,-1.2085227872,0.000058382
$\mathrm{H}, 0.4684473021,-2.1493318996,0.0001176642$
C, $-1.472145219,1.2084486925,0.0000614546$
$\mathrm{H}, 0.4684186334,2.1492792703,0.0001128647$
C,-2.1721690243,-0.000059042,0.0000678104
$\mathrm{H},-2.0124879837,-2.1505117186,-0.0000153511$ 
$\mathrm{H},-2.0125633866,2.1504093259,-0.0000125211$

$\mathrm{H},-3.2581304719,-0.0000673216,-0.0000126434$

C,3.2744563178,0.0001005295,0.000191878

$\mathrm{H}, 4.339766808,0.0001449636,0.0001733283$

C,2.0643814067,0.0000232146,0.0002186743

Zero-point correction=

Thermal correction to Energy=

Thermal correction to Enthalpy=

Thermal correction to Gibbs Free Energy=

Sum of electronic and zero-point Energies=

Sum of electronic and thermal Energies=

Sum of electronic and thermal Enthalpies=

Sum of electronic and thermal Free Energies=
0.109451 (Hartree/Particle)

0.115915

0.116859

0.079024

$-308.293983$

$-308.287519$

$-308.286575$

$-308.324410$

Table S75. Cartesian coordinates and energy values for the $\mathbf{C N}-\mathbf{P h}$.

C,2.0833471533,0.6166561998,0.5926578657

$\mathrm{N}, 3.052113557,0.0287270595,0.258569469$

C,4.1882991664,-0.6608610504,-0.1331233033

C,5.0287064673,-0.1132886794,-1.1109534542

C,4.4854492992,-1.8977982594,0.4537357224

C,6.169410336,-0.8121971423,-1.4989034564

$\mathrm{H}, 4.7787462555,0.8457902189,-1.550845209$

C,5.6296767689,-2.5844386495,0.0551315393

$\mathrm{H}, 3.8202252665,-2.3014028119,1.2089944477$

C,6.4726963828,-2.0459451534,-0.919382539

$H, 6.8225707162,-0.3905066084,-2.2565119689$

$H, 5.8624046762,-3.5431719675,0.5079855688$

H,7.3631497746,-2.585775706,-1.225978752

Zero-point correction=

Thermal correction to Energy=

Thermal correction to Enthalpy=

Thermal correction to Gibbs Free Energy=

Sum of electronic and zero-point Energies=

Sum of electronic and thermal Energies=

Sum of electronic and thermal Enthalpies=

Sum of electronic and thermal Free Energies=
0.098609 (Hartree/Particle)

0.104837

0.105781
0.068285

$-324.367171$

$-324.360943$

$-324.359999$

$-324.397495$

Table S76. Cartesian coordinates and energy values for the NHC ${ }^{\text {iPr2Me2 }}$.

$\mathrm{N}, 10.6155410148,6.0051547239,2.3570170272$

$\mathrm{N}, 11.6488467321,6.2252888759,4.2168676414$

$\mathrm{C}, 10.4584669622,5.7859325879,3.7002345168$

C,11.8612748791,6.5705121205,2.0416083281

C, $12.5218447793,6.7113732206,3.2307114954$

C,9.5013871357,5.6749804311,1.4512809507

C,9.8797846414,4.6375570926,0.3870520628

C,12.3426712692,6.9052861888,0.6645686127

C,13.9022839048,7.2371703356,3.4716035383

C,11.8478781641,6.1765270976,5.6758798001

C,13.050072226,5.3190730779,6.0900194382

C,11.8659020503,7.5735738443,6.3096909453

$\mathrm{H}, 8.7835076349,5.2117145255,2.1334171835$

$\mathrm{H}, 8.9747574171,4.2825222103,-0.1165270243$

$\mathrm{H}, 10.3736800732,3.7767315861,0.8466636728$

$\mathrm{H}, 10.5413175637,5.0474394915,-0.381318931$ 
$\mathrm{H}, 11.646029395,7.5534422094,0.1233144802$

$\mathrm{H}, 12.5033930878,6.0125086401,0.0493948617$

$\mathrm{H}, 13.2972273115,7.4340841864,0.7189084509$

$\mathrm{H}, 14.3114390362,7.6544539552,2.5484445479$

$\mathrm{H}, 14.5943840833,6.4561729664,3.8069003678$

$\mathrm{H}, 13.9219166812,8.0344036469,4.2215051191$

$\mathrm{H}, 10.9398670444,5.6690763902,6.0124574594$

$\mathrm{H}, 13.0248255591,5.1510988852,7.171615363$

$\mathrm{H}, 14.0058489435,5.7963379534,5.8561209517$

$\mathrm{H}, 13.0180615919,4.3451400575,5.5933899862$

$\mathrm{H}, 11.8489980864,7.4868490591,7.4012003544$

$\mathrm{H}, 10.9867068993,8.1438768843,5.9976547288$

$\mathrm{H}, 12.7603151436,8.1433124901,6.0396317637$

C,8.8418304853,6.9257536509,0.8560337736

$\mathrm{H}, 9.4892370705,7.4378121092,0.1375963577$

$\mathrm{H}, 7.9233298743,6.6464597073,0.3292112361$

$\mathrm{H}, 8.580666939,7.6318178382,1.6490480296$

Zero-point correction $=$

Thermal correction to Energy=

Thermal correction to Enthalpy=

0.296140 (Hartree/Particle)

Thermal correction to Gibbs Free Energy= 0.312480

Sum of electronic and zero-point Energies=

0.311536

0.312480

Sum of electronic and thermal Energies=

238

$-540.420721$

Sum of electronic and thermal Enthalpies=

$-540.405325$

Sum of electronic and thermal Free Energies $=$

$-540.404381$

$E$ in a.u.: 540.71686095

$-540.462623$ 
Table S77. Cartesian coordinates and energy value for the 9 .

\begin{tabular}{|c|c|c|c|c|c|}
\hline \multirow{2}{*}{$\begin{array}{l}\text { Center } \\
\text { Number }\end{array}$} & \multirow{2}{*}{$\begin{array}{l}\text { Atomic } \\
\text { Number }\end{array}$} & \multicolumn{2}{|c|}{ Atomic } & \multicolumn{2}{|c|}{ Coordinates (Angstroms) } \\
\hline & & & Type & Y & Z \\
\hline 1 & 32 & 0 & -0.274808 & -2.125389 & 0.029584 \\
\hline 2 & 14 & 0 & 1.123367 & 0.908116 & 0.668220 \\
\hline 3 & 14 & 0 & -1.258510 & 0.099820 & 0.422164 \\
\hline 4 & 7 & 0 & -0.766908 & -2.419979 & -3.121765 \\
\hline 5 & 7 & 0 & 1.158612 & -3.114626 & -2.399077 \\
\hline 6 & 6 & 0 & 0.313758 & 0.961598 & 2.378902 \\
\hline 7 & 1 & 0 & 0.741441 & 1.225711 & 3.348986 \\
\hline 8 & 6 & 0 & -0.959428 & 0.495770 & 2.285003 \\
\hline 9 & 6 & 0 & -1.837297 & 0.332711 & 3.474472 \\
\hline 10 & 6 & 0 & -3.175608 & 0.767845 & 3.490472 \\
\hline 11 & 6 & 0 & -3.957989 & 0.646147 & 4.639226 \\
\hline 12 & 6 & 0 & -3.433792 & 0.069280 & 5.796366 \\
\hline 13 & 6 & 0 & -2.113167 & -0.381837 & 5.794637 \\
\hline 14 & 6 & 0 & -1.326487 & -0.246848 & 4.651854 \\
\hline 15 & 6 & 0 & -0.027704 & -2.574147 & -1.991850 \\
\hline 16 & 6 & 0 & -0.044695 & -2.837145 & -4.240664 \\
\hline 17 & 6 & 0 & 1.159539 & -3.298070 & -3.780329 \\
\hline 18 & 6 & 0 & -2.199589 & -2.027689 & -3.083022 \\
\hline 19 & 6 & 0 & -2.431277 & -0.589415 & -3.549524 \\
\hline 20 & 6 & 0 & -3.078188 & -3.044411 & -3.824161 \\
\hline 21 & 6 & 0 & -0.513756 & -2.760497 & -5.661420 \\
\hline 22 & 6 & 0 & 2.253976 & -3.923449 & -4.587061 \\
\hline 23 & 6 & 0 & 2.182971 & -3.556101 & -1.401684 \\
\hline 24 & 6 & 0 & 1.794519 & -4.925346 & -0.838581 \\
\hline 25 & 6 & 0 & 3.629291 & -3.526363 & -1.893310 \\
\hline 26 & 6 & 0 & -2.997055 & 0.950848 & 0.022291 \\
\hline 27 & 6 & 0 & -4.184250 & 0.166572 & -0.136663 \\
\hline 28 & 6 & 0 & -5.411077 & 0.792106 & -0.397381 \\
\hline 29 & 6 & 0 & -5.549925 & 2.176214 & -0.483464 \\
\hline 30 & 6 & 0 & -4.408189 & 2.939134 & -0.258897 \\
\hline 31 & 6 & 0 & -3.153634 & 2.369241 & 0.000373 \\
\hline 32 & 6 & 0 & 2.804753 & -0.170087 & 0.740988 \\
\hline 33 & 6 & 0 & 3.012094 & -1.156681 & 1.774018 \\
\hline 34 & 6 & 0 & 4.256059 & -1.782644 & 1.926338 \\
\hline 35 & 6 & 0 & 5.358531 & -1.511520 & 1.121241 \\
\hline 36 & 6 & 0 & 5.154710 & -0.595492 & 0.100820 \\
\hline 37 & 6 & 0 & 3.932262 & 0.060698 & -0.118667 \\
\hline 38 & 6 & 0 & 1.998531 & -1.589411 & 2.846946 \\
\hline 39 & 6 & 0 & 1.783824 & -3.114894 & 2.923459 \\
\hline 40 & 6 & 0 & 2.430419 & -1.049644 & 4.226995 \\
\hline 41 & 6 & 0 & 6.720189 & -2.148280 & 1.358954 \\
\hline 42 & 6 & 0 & 6.675141 & -3.685974 & 1.303226 \\
\hline 43 & 6 & 0 & 7.338155 & -1.667165 & 2.686261 \\
\hline 44 & 6 & 0 & 3.977902 & 0.996967 & -1.334078 \\
\hline 45 & 6 & 0 & 4.276098 & 0.232138 & -2.639902 \\
\hline 46 & 6 & 0 & 4.998909 & 2.139957 & -1.169700 \\
\hline 47 & 6 & 0 & 1.552066 & 2.657901 & -0.029432 \\
\hline 48 & 6 & 0 & 2.286834 & 3.564956 & 0.780025 \\
\hline 49 & 6 & 0 & 2.645282 & 4.826033 & 0.279753 \\
\hline 50 & 6 & 0 & 2.345117 & 5.217979 & -1.018820 \\
\hline 51 & 6 & 0 & 1.623280 & 4.321778 & -1.815872 \\
\hline 52 & 6 & 0 & 1.194685 & 3.072326 & -1.355391 \\
\hline 53 & 6 & 0 & 2.691480 & 3.253543 & 2.225623 \\
\hline 54 & 6 & 0 & 4.176867 & 3.524210 & 2.531437 \\
\hline 55 & 6 & 0 & 1.792113 & 4.017798 & 3.217083 \\
\hline 56 & 6 & 0 & 2.783803 & 6.576224 & -1.549840 \\
\hline
\end{tabular}




\begin{tabular}{|c|c|c|c|c|c|}
\hline 57 & 1 & 0 & 3.303367 & 7.088069 & -0.728915 \\
\hline 58 & 6 & 0 & 3.784876 & 6.437744 & -2.712484 \\
\hline 59 & 1 & 0 & 4.657974 & 5.849121 & -2.414097 \\
\hline 60 & 1 & 0 & 3.326007 & 5.940356 & -3.574092 \\
\hline 61 & 1 & 0 & 4.133958 & 7.421965 & -3.044070 \\
\hline 62 & 6 & 0 & 1.585820 & 7.455200 & -1.955041 \\
\hline 63 & 1 & 0 & 0.890064 & 7.584826 & -1.120326 \\
\hline 64 & 1 & 0 & 1.924123 & 8.447135 & -2.274519 \\
\hline 65 & 1 & 0 & 1.029252 & 7.010872 & -2.787214 \\
\hline 66 & 6 & 0 & 0.318017 & 2.218579 & -2.299480 \\
\hline 67 & 6 & 0 & -0.183443 & 2.968098 & -3.548657 \\
\hline 68 & 6 & 0 & 0.963660 & 0.905790 & -2.786087 \\
\hline 69 & 1 & 0 & -3.600889 & 1.214691 & 2.600286 \\
\hline 70 & 1 & 0 & -4.984431 & 1.002162 & 4.62555 \\
\hline 71 & 1 & 0 & -4.047248 & -0.030139 & 6.687246 \\
\hline 72 & 1 & 0 & -1.691703 & -0.839333 & 6.68575 \\
\hline 73 & 1 & 0 & -0.302134 & -0.600508 & $4.66171 \mathrm{C}$ \\
\hline 74 & 1 & 0 & -2.441675 & -2.081158 & -2.02049 \\
\hline 75 & 1 & 0 & -3.489671 & -0.338079 & -3.439230 \\
\hline 76 & 1 & 0 & -2.157709 & -0.442776 & -4.598943 \\
\hline 77 & 1 & 0 & -1.861308 & 0.107764 & -2.93451 \\
\hline 78 & 1 & 0 & -4.120127 & -2.882447 & -3.54324 \\
\hline 79 & 1 & 0 & -2.815804 & -4.070207 & -3.55082 \\
\hline 80 & 1 & 0 & -3.015306 & -2.943092 & -4.90934 \\
\hline 81 & 1 & 0 & -1.058924 & -1.835648 & -5.86099 \\
\hline 82 & 1 & 0 & -1.164070 & -3.596922 & -5.93811 \\
\hline 83 & 1 & 0 & 0.345831 & -2.775969 & -6.33438 \\
\hline 84 & 1 & 0 & 1.932317 & -4.011570 & -5.62613 \\
\hline 85 & 1 & 0 & 2.489724 & -4.932869 & -4.23696 \\
\hline 86 & 1 & 0 & 3.178581 & -3.341048 & -4.57251 \\
\hline 87 & 1 & 0 & 2.088021 & -2.811435 & -0.60477 \\
\hline 88 & 1 & 0 & 2.507725 & -5.211868 & -0.05963 \\
\hline 89 & 1 & 0 & 1.812751 & -5.697560 & -1.61637 \\
\hline 90 & 1 & 0 & 0.798202 & -4.899959 & -0.39156 \\
\hline 91 & 1 & 0 & 4.269113 & -3.573343 & -1.00862 \\
\hline 92 & 1 & 0 & 3.873125 & -2.598075 & -2.41125 \\
\hline 93 & 1 & 0 & 3.885542 & -4.375330 & -2.53146 \\
\hline 94 & 1 & 0 & -6.293942 & 0.171074 & -0.51598 \\
\hline 95 & 1 & 0 & -4.499889 & 4.021776 & -0.27562 \\
\hline 96 & 1 & 0 & 4.370306 & -2.496495 & 2.73619 \\
\hline 97 & 1 & 0 & 5.988571 & -0.369721 & -0.558510 \\
\hline 98 & 1 & 0 & 1.025708 & -1.174668 & 2.60246 \\
\hline 99 & 1 & 0 & 2.701318 & -3.667678 & 3.14898 \\
\hline 100 & 1 & 0 & 1.065481 & -3.340744 & 3.7197 \\
\hline 101 & 1 & 0 & 1.361586 & -3.490726 & 1.9886 \\
\hline 102 & 1 & U & 1.699614 & -1.315791 & 4.9989 \\
\hline 103 & 1 & 0 & 3.392912 & -1.471860 & 4.5347 \\
\hline 104 & 1 & 0 & 2.539155 & 0.038991 & 4.22247 \\
\hline 105 & 1 & U & 7.378808 & -1.808625 & 0.54807 \\
\hline 106 & 1 & 0 & 7.676254 & -4.107440 & 1.4462 \\
\hline 107 & 1 & 0 & 6.295619 & -4.039125 & 0.33896 \\
\hline 108 & 1 & 0 & 6.029262 & -4.095920 & 2.0869 \\
\hline 109 & 1 & 0 & 8.345378 & -2.078035 & 2.818 \\
\hline 110 & 1 & 0 & 7.407254 & -0.575593 & 2.7162 \\
\hline 111 & 1 & 0 & 6.732534 & -1.985799 & 3.54163 \\
\hline 112 & 1 & 0 & 3.009574 & 1.464825 & $-1.4508^{\circ}$ \\
\hline 113 & 1 & 0 & 3.522260 & -0.534594 & -2.8376 \\
\hline 114 & 1 & 0 & 5.255278 & -0.256807 & -2.6133 \\
\hline 115 & 1 & 0 & 4.281009 & 0.922622 & -3.4914 \\
\hline 116 & 1 & 0 & 4.956784 & 2.808324 & -2.0369 \\
\hline 117 & 1 & 0 & 6.024797 & 1.764558 & -1.090 \\
\hline
\end{tabular}

\section{S-124}




\begin{tabular}{|c|c|c|c|c|c|}
\hline 118 & 1 & 0 & 4.786525 & 2.737318 & -0.282446 \\
\hline 119 & 1 & 0 & 3.190413 & 5.515753 & 0.919118 \\
\hline 120 & 1 & 0 & 1.376620 & 4.627891 & -2.825405 \\
\hline 121 & 1 & 0 & 2.542905 & 2.185547 & 2.397778 \\
\hline 122 & 1 & 0 & 4.439453 & 4.582019 & 2.425913 \\
\hline 123 & 1 & 0 & 4.399431 & 3.236964 & 3.564896 \\
\hline 124 & 1 & 0 & 4.833575 & 2.944723 & 1.877166 \\
\hline 125 & 1 & 0 & 2.045281 & 3.758420 & 4.251408 \\
\hline 126 & 1 & 0 & 1.918843 & 5.100499 & 3.103922 \\
\hline 127 & 1 & 0 & 0.736470 & 3.784552 & 3.056198 \\
\hline 128 & 1 & 0 & -0.570823 & 1.937914 & -1.721884 \\
\hline 129 & 1 & 0 & 0.628010 & 3.157190 & -4.261197 \\
\hline 130 & 1 & 0 & -0.928396 & 2.354521 & -4.065225 \\
\hline 131 & 1 & 0 & -0.651472 & 3.925207 & -3.310065 \\
\hline 132 & 1 & 0 & 1.143652 & 0.184815 & -1.983128 \\
\hline 133 & 1 & 0 & 1.903253 & 1.102667 & -3.305005 \\
\hline 134 & 1 & 0 & 0.295850 & 0.417694 & -3.502115 \\
\hline 135 & 6 & 0 & -1.532473 & -3.604795 & 0.524064 \\
\hline 136 & 6 & 0 & -2.058934 & -4.626361 & -0.284757 \\
\hline 137 & 6 & 0 & -1.820829 & -3.672152 & 1.901458 \\
\hline 138 & 6 & 0 & -2.826314 & -5.666308 & 0.250325 \\
\hline 139 & 1 & 0 & -1.860018 & -4.629023 & -1.354148 \\
\hline 140 & 6 & 0 & -2.593401 & -4.696810 & 2.444650 \\
\hline 141 & 1 & 0 & -1.437080 & -2.889409 & 2.553842 \\
\hline 142 & 6 & 0 & -3.099143 & -5.704692 & 1.617902 \\
\hline 143 & 1 & 0 & -3.214257 & -6.444753 & -0.402783 \\
\hline 144 & 1 & 0 & -2.800265 & -4.712015 & 3.511654 \\
\hline 145 & 1 & 0 & -3.696401 & -6.509988 & 2.036306 \\
\hline 146 & 6 & 0 & -2.032371 & 3.356905 & 0.330557 \\
\hline 147 & 6 & 0 & -4.254128 & -1.349566 & 0.076567 \\
\hline 148 & 1 & 0 & -3.246337 & -1.766681 & 0.026989 \\
\hline 149 & 6 & 0 & -2.184037 & 3.921164 & 1.758284 \\
\hline 150 & 1 & 0 & -1.376903 & 4.631275 & 1.969805 \\
\hline 151 & 1 & 0 & -3.135541 & 4.454460 & 1.865949 \\
\hline 152 & 1 & 0 & -2.149947 & 3.135966 & 2.515171 \\
\hline 153 & 6 & 0 & -5.121872 & -2.077493 & -0.969894 \\
\hline 154 & 1 & 0 & -4.907995 & -3.150868 & -0.950642 \\
\hline 155 & 1 & 0 & -6.191290 & -1.959658 & -0.763659 \\
\hline 156 & 1 & 0 & -4.947676 & -1.702294 & -1.981871 \\
\hline 157 & 6 & 0 & -4.795378 & -1.687212 & 1.481675 \\
\hline 158 & 1 & 0 & -4.863166 & -2.772070 & 1.609305 \\
\hline 159 & 1 & 0 & -4.151340 & -1.296247 & 2.270573 \\
\hline 160 & 1 & 0 & -5.795774 & -1.260904 & 1.622763 \\
\hline 161 & 6 & 0 & -6.890212 & 2.833863 & -0.781214 \\
\hline 162 & 1 & 0 & -6.721958 & 3.919081 & -0.782086 \\
\hline 163 & 6 & 0 & -7.417037 & 2.447603 & -2.175906 \\
\hline 164 & 1 & 0 & -8.353174 & 2.972092 & -2.397757 \\
\hline 165 & 1 & 0 & -6.691805 & 2.699970 & -2.955883 \\
\hline 166 & 1 & 0 & -7.614907 & 1.372002 & -2.239958 \\
\hline 167 & 6 & 0 & -7.937084 & 2.531469 & 0.307160 \\
\hline 168 & 1 & 0 & -8.168785 & 1.461753 & 0.350592 \\
\hline 169 & 1 & 0 & -7.577913 & 2.834319 & 1.295452 \\
\hline 170 & 1 & 0 & -8.872161 & 3.065684 & 0.104962 \\
\hline 171 & 6 & 0 & -1.948853 & 4.529432 & -0.665356 \\
\hline 172 & 1 & 0 & -1.006420 & 5.068428 & -0.534600 \\
\hline 173 & 1 & 0 & -2.007424 & 4.186499 & -1.700673 \\
\hline 174 & 1 & 0 & -2.760868 & 5.248317 & -0.510738 \\
\hline 175 & 1 & 0 & -1.079489 & 2.824116 & 0.295928 \\
\hline
\end{tabular}

E in a.u.: 5491.35219097 
Table S78. Cartesian coordinates and energy values for the 10.

\begin{tabular}{|c|c|c|c|c|c|}
\hline \multirow{2}{*}{$\begin{array}{l}\text { Center } \\
\text { Number }\end{array}$} & \multirow{2}{*}{\multicolumn{2}{|c|}{$\begin{array}{l}\text { Atomic } \\
\text { Number }\end{array}$}} & \multirow{2}{*}{$\begin{array}{l}\text { Atomic } \\
\text { Type }\end{array}$} & \multicolumn{2}{|c|}{ Coordinates (Angstroms) } \\
\hline & & & & $\mathrm{X}$ & Z \\
\hline 1 & 32 & 0 & 1.873755 & -1.063756 & -1.327336 \\
\hline 2 & 14 & 0 & -0.164817 & 1.110595 & 0.619777 \\
\hline 3 & 14 & 0 & -0.036754 & -1.253828 & 0.261074 \\
\hline 4 & 6 & 0 & 0.183253 & 0.349117 & 2.320148 \\
\hline 5 & 1 & 0 & 0.333188 & 0.833577 & 3.288602 \\
\hline 6 & 6 & 0 & 0.375111 & -0.988429 & 2.131772 \\
\hline 7 & 6 & 0 & 0.921426 & -1.930932 & 3.137405 \\
\hline 8 & 6 & 0 & 0.989585 & -3.308429 & 2.859732 \\
\hline 9 & 6 & 0 & 1.520618 & -4.212743 & 3.778986 \\
\hline 10 & 6 & 0 & 1.999674 & -3.759893 & 5.008030 \\
\hline 11 & 6 & 0 & 1.941299 & -2.395607 & 5.305353 \\
\hline 12 & 6 & 0 & 1.412868 & -1.495808 & 4.383966 \\
\hline 13 & 6 & 0 & -1.534611 & -2.390157 & -0.224277 \\
\hline 14 & 6 & 0 & -1.761665 & -2.675760 & -1.609620 \\
\hline 15 & 6 & 0 & -2.868687 & -3.431999 & -2.002339 \\
\hline 16 & 6 & O & -3.799180 & -3.930192 & -1.087445 \\
\hline 17 & 6 & 0 & -3.570711 & -3.660823 & 0.256661 \\
\hline 18 & 6 & 0 & -2.468231 & -2.922029 & 0.712936 \\
\hline 19 & 6 & 0 & 1.461824 & 1.803148 & -0.238371 \\
\hline 20 & 6 & 0 & 2.766457 & 1.839344 & 0.353779 \\
\hline 21 & 6 & 0 & 3.879178 & 2.208515 & -0.416414 \\
\hline 22 & 6 & 0 & 3.779853 & 2.613386 & -1.744972 \\
\hline 23 & 6 & 0 & 2.509550 & 2.608001 & -2.313441 \\
\hline 24 & 6 & 0 & 1.375388 & 2.190368 & -1.612158 \\
\hline 25 & 6 & 0 & 3.078819 & 1.510467 & 1.816465 \\
\hline 26 & 6 & 0 & 3.973579 & 0.262462 & 1.935135 \\
\hline 27 & 6 & 0 & 3.706783 & 2.704523 & 2.562152 \\
\hline 28 & 6 & 0 & 4.997916 & 3.044997 & -2.548294 \\
\hline 29 & 6 & 0 & 6.021777 & 1.904319 & -2.695369 \\
\hline 30 & 6 & 0 & 5.655425 & 4.306266 & -1.957547 \\
\hline 31 & 6 & 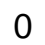 & 0.067793 & 2.163786 & -2.400163 \\
\hline 32 & 6 & 0 & 0.215883 & 1.476978 & -3.773353 \\
\hline 33 & 6 & 0 & -0.503665 & 3.585004 & -2.563911 \\
\hline 34 & 6 & 0 & -1.587972 & 2.411639 & 0.625950 \\
\hline 35 & 6 & 0 & -1.361521 & 3.636759 & 1.313794 \\
\hline 36 & 6 & 0 & -2.347292 & 4.630882 & 1.327536 \\
\hline 37 & 6 & 0 & -3.571728 & 4.476026 & 0.681769 \\
\hline 38 & 6 & 0 & -3.797049 & 3.267382 & 0.022900 \\
\hline 39 & 6 & 0 & -2.848744 & 2.237634 & -0.014810 \\
\hline 40 & 6 & 0 & -0.071039 & 3.940333 & 2.076462 \\
\hline 41 & 6 & 0 & 0.661503 & 5.168869 & 1.505363 \\
\hline 42 & 6 & 0 & -0.328782 & 4.096050 & 3.587845 \\
\hline 43 & 6 & 0 & -4.618146 & 5.581461 & 0.711351 \\
\hline 44 & 1 & 0 & -4.178097 & 6.424080 & 1.261231 \\
\hline 45 & 6 & 0 & -4.964383 & 6.084509 & -0.702044 \\
\hline 46 & 1 & 0 & -4.070413 & 6.433253 & -1.228045 \\
\hline 47 & 1 & 0 & -5.418660 & 5.292100 & -1.306699 \\
\hline 48 & 1 & 0 & -5.677959 & 6.914283 & -0.652532 \\
\hline 49 & 6 & 0 & -5.886118 & 5.148233 & 1.471062 \\
\hline 50 & 1 & 0 & -5.646760 & 4.826936 & 2.489384 \\
\hline 51 & 1 & 0 & -6.601684 & 5.975526 & 1.532786 \\
\hline 52 & 1 & 0 & -6.385296 & 4.313258 & 0.967554 \\
\hline 53 & 6 & 0 & -3.271533 & 0.957410 & -0.739215 \\
\hline 54 & 6 & 0 & -4.514521 & 0.322520 & -0.083725 \\
\hline
\end{tabular}




\begin{tabular}{|c|c|c|c|c|c|}
\hline 55 & 6 & 0 & -3.553033 & 1.180243 & -2.238231 \\
\hline 56 & 1 & 0 & 0.604592 & -3.674701 & 1.912132 \\
\hline 57 & 1 & 0 & 1.555904 & -5.270697 & 3.535121 \\
\hline 58 & 1 & 0 & 2.415302 & -4.459942 & 5.726730 \\
\hline 59 & 1 & 0 & 2.314676 & -2.031172 & 6.258299 \\
\hline 60 & 1 & 0 & 1.390419 & -0.439266 & 4.630439 \\
\hline 61 & 1 & 0 & -3.015184 & -3.638081 & -3.056996 \\
\hline 62 & 1 & 0 & -4.278738 & -4.042389 & 0.987614 \\
\hline 63 & 1 & 0 & 4.856494 & 2.202889 & 0.057488 \\
\hline 64 & 1 & 0 & 2.404409 & 2.914854 & -3.349967 \\
\hline 65 & 1 & 0 & 2.144258 & 1.280214 & 2.322506 \\
\hline 66 & 1 & 0 & 4.977210 & 0.452193 & 1.538674 \\
\hline 67 & 1 & 0 & 4.080132 & -0.044061 & 2.980787 \\
\hline 68 & 1 & 0 & 3.556978 & -0.574658 & 1.371455 \\
\hline 69 & 1 & 0 & 3.841812 & 2.459296 & 3.621654 \\
\hline 70 & 1 & 0 & 4.690731 & 2.965891 & 2.159185 \\
\hline 71 & 1 & 0 & 3.076810 & 3.596312 & 2.497736 \\
\hline 72 & 1 & 0 & 4.643022 & 3.299782 & -3.555724 \\
\hline 73 & 1 & 0 & 6.859757 & 2.216287 & -3.328403 \\
\hline 74 & 1 & 0 & 5.562872 & 1.019291 & -3.146680 \\
\hline 75 & 1 & 0 & 6.430995 & 1.608087 & -1.723517 \\
\hline 76 & 1 & 0 & 6.492323 & 4.635540 & -2.583164 \\
\hline 77 & 1 & 0 & 4.938724 & 5.130129 & -1.885540 \\
\hline 78 & 1 & 0 & 6.046837 & 4.116185 & -0.952249 \\
\hline 79 & 1 & 0 & -0.657777 & 1.574952 & -1.830332 \\
\hline 80 & 1 & 0 & 0.742079 & 0.524229 & -3.668967 \\
\hline 81 & 1 & 0 & 0.774245 & 2.098542 & -4.480859 \\
\hline 82 & 1 & 0 & -0.769215 & 1.294290 & -4.215509 \\
\hline 83 & 1 & 0 & -1.446276 & 3.567287 & -3.121832 \\
\hline 84 & 1 & 0 & 0.199842 & 4.216058 & -3.118426 \\
\hline 85 & 1 & 0 & -0.693594 & 4.055737 & -1.596256 \\
\hline 86 & 1 & 0 & -2.154900 & 5.558654 & 1.860806 \\
\hline 87 & 1 & 0 & -4.748930 & 3.113243 & -0.478007 \\
\hline 88 & 1 & 0 & 0.607654 & 3.092357 & 1.957087 \\
\hline 89 & 1 & 0 & 0.050649 & 6.074165 & 1.583283 \\
\hline 90 & 1 & 0 & 1.592240 & 5.353402 & 2.052967 \\
\hline 91 & 1 & 0 & 0.913078 & 5.020244 & 0.451368 \\
\hline 92 & 1 & 0 & 0.614572 & 4.237524 & 4.126990 \\
\hline 93 & 1 & 0 & -0.964423 & 4.962404 & 3.799333 \\
\hline 94 & 1 & 0 & -0.828458 & 3.212488 & 3.997305 \\
\hline 95 & 1 & 0 & -2.455290 & 0.235515 & -0.659439 \\
\hline 96 & 1 & 0 & -5.410170 & 0.925992 & -0.268803 \\
\hline 97 & 1 & 0 & -4.692095 & -0.676704 & -0.493910 \\
\hline 98 & 1 & 0 & -4.398901 & 0.229740 & 0.997429 \\
\hline 99 & 1 & 0 & -2.687104 & 1.584873 & -2.766110 \\
\hline 100 & 1 & 0 & -4.386181 & 1.875378 & -2.388646 \\
\hline 101 & 1 & 0 & -3.827954 & 0.231424 & -2.711669 \\
\hline 102 & 6 & 0 & 3.380199 & -2.258336 & -0.844822 \\
\hline 103 & 6 & 0 & 3.462551 & -3.243364 & 0.155865 \\
\hline 104 & 6 & 0 & 4.498640 & -2.102614 & -1.688561 \\
\hline 105 & 6 & 0 & 4.599944 & -4.034251 & 0.309881 \\
\hline 106 & 1 & 0 & 2.632848 & -3.384179 & 0.838715 \\
\hline 107 & 6 & 0 & 5.643625 & -2.885313 & -1.540964 \\
\hline 108 & 1 & 0 & 4.470429 & -1.352927 & -2.476869 \\
\hline 109 & 6 & 0 & 5.694679 & -3.856332 & -0.540231 \\
\hline 110 & 1 & 0 & 4.636169 & -4.785697 & 1.094111 \\
\hline 111 & 1 & 0 & 6.492796 & -2.742405 & -2.203822 \\
\hline 112 & 1 & 0 & 6.583245 & -4.470293 & -0.420797 \\
\hline 113 & 6 & 0 & -2.364736 & -2.765760 & 2.232071 \\
\hline 114 & 6 & 0 & -0.805496 & -2.210249 & -2.709955 \\
\hline 115 & 1 & 0 & -0.376592 & -1.247908 & -2.400699 \\
\hline
\end{tabular}




$\begin{array}{rlrrrr}116 & 6 & 0 & -2.411694 & -4.124479 & 2.959678 \\ 117 & 1 & 0 & -2.173399 & -3.989438 & 4.019785 \\ 118 & 1 & 0 & -3.401692 & -4.589441 & 2.902345 \\ 119 & 1 & 0 & -1.688669 & -4.830126 & 2.540192 \\ 120 & 6 & 0 & -1.465321 & -1.940978 & -4.073241 \\ 121 & 1 & 0 & -0.745413 & -1.454973 & -4.738689 \\ 122 & 1 & 0 & -1.784612 & -2.864291 & -4.568007 \\ 123 & 1 & 0 & -2.336045 & -1.285842 & -3.980022 \\ 124 & 6 & 0 & 0.360257 & -3.205844 & -2.895159 \\ 125 & 1 & 0 & 1.092780 & -2.813981 & -3.609767 \\ 126 & 1 & 0 & 0.885629 & -3.417478 & -1.955294 \\ 127 & 1 & 0 & -0.010534 & -4.166149 & -3.270727 \\ 128 & 6 & 0 & -5.008035 & -4.740272 & -1.533775 \\ 129 & 1 & 0 & -5.571796 & -4.999189 & -0.627775 \\ 130 & 6 & 0 & -5.944498 & -3.921749 & -2.442018 \\ 131 & 1 & 0 & -6.832518 & -4.505201 & -2.708915 \\ 132 & 1 & 0 & -6.275903 & -3.004793 & -1.944913 \\ 133 & 1 & 0 & -5.444234 & -3.634101 & -3.373097 \\ 134 & 6 & 0 & -4.595133 & -6.060396 & -2.211683 \\ 135 & 1 & 0 & -4.041768 & -5.876108 & -3.138995 \\ 136 & 1 & 0 & -3.955627 & -6.658883 & -1.555663 \\ 137 & 1 & 0 & -5.477935 & -6.657391 & -2.465508 \\ 138 & 6 & 0 & -3.423168 & -1.808867 & 2.808876 \\ 139 & 1 & 0 & -4.440341 & -2.122280 & 2.549755 \\ 140 & 1 & 0 & -3.349105 & -1.776634 & 3.901609 \\ 141 & 1 & 0 & -3.274945 & -0.792784 & 2.435512 \\ 142 & 1 & 0 & -1.401245 & -2.323007 & 2.461824 \\ & 1 & & & \end{array}$

E in a.u.: 4950.60974224

Table S79. Cartesian coordinates and energy values for the TS (from 9 to $10+$ NHC).

\begin{tabular}{|c|c|c|c|c|c|}
\hline \multirow{2}{*}{$\begin{array}{l}\text { Center } \\
\text { Number }\end{array}$} & \multirow{2}{*}{$\begin{array}{l}\text { Atomic } \\
\text { Number }\end{array}$} & \multicolumn{2}{|c|}{ Atomic } & \multicolumn{2}{|c|}{ Coordinates (Angstroms) } \\
\hline & & & & $\begin{array}{ll}X & Y\end{array}$ & Z \\
\hline 1 & 32 & 0 & -0.020750 & -1.975852 & 0.400474 \\
\hline 2 & 14 & 0 & 0.844530 & 1.308608 & 0.569351 \\
\hline 3 & 14 & 0 & -1.363763 & 0.090457 & 0.405718 \\
\hline 4 & 7 & 0 & 0.225728 & -3.673265 & -3.049437 \\
\hline 5 & 7 & 0 & 2.092151 & -3.743137 & -1.943081 \\
\hline 6 & 6 & 0 & -0.044304 & 1.494724 & 2.230704 \\
\hline 7 & 1 & 0 & 0.288904 & 1.974076 & 3.154244 \\
\hline 8 & 6 & 0 & -1.221132 & 0.816581 & 2.185829 \\
\hline 9 & 6 & 0 & -2.117834 & 0.699082 & 3.366591 \\
\hline 10 & 6 & 0 & -3.507552 & 0.904799 & 3.282704 \\
\hline 11 & 6 & 0 & -4.314726 & 0.836949 & 4.418676 \\
\hline 12 & 6 & 0 & -3.761453 & 0.542454 & 5.665148 \\
\hline 13 & 6 & 0 & -2.387315 & 0.320489 & 5.765319 \\
\hline 14 & 6 & 0 & -1.578303 & 0.402924 & 4.633177 \\
\hline 15 & 6 & 0 & 0.794780 & -3.323405 & -1.865935 \\
\hline 16 & 6 & 0 & 1.162575 & -4.288698 & -3.880770 \\
\hline 17 & 6 & 0 & 2.332148 & -4.354377 & -3.172022 \\
\hline 18 & 6 & 0 & -1.235461 & -3.553261 & -3.291294 \\
\hline 19 & 6 & 0 & -1.585098 & -2.405346 & -4.239966 \\
\hline 20 & 6 & 0 & -1.842240 & -4.893693 & -3.727195 \\
\hline 21 & 6 & 0 & 0.913490 & -4.754954 & -5.282556 \\
\hline 22 & 6 & 0 & 3.613089 & -4.997087 & -3.603096 \\
\hline 23 & 6 & 0 & 2.999791 & -3.651681 & -0.756983 \\
\hline 24 & 6 & 0 & 2.718250 & -4.815958 & 0.195911 \\
\hline
\end{tabular}




\begin{tabular}{|c|c|c|c|c|c|}
\hline 25 & 6 & 0 & 4.487257 & -3.521013 & -1.080697 \\
\hline 26 & 6 & 0 & -3.194416 & 0.570222 & -0.164564 \\
\hline 27 & 6 & 0 & -4.232645 & -0.414044 & -0.220136 \\
\hline 28 & 6 & 0 & -5.529476 & -0.047795 & -0.605445 \\
\hline 29 & 6 & 0 & -5.882574 & 1.263792 & -0.917706 \\
\hline 30 & 6 & 0 & -4.888916 & 2.230026 & -0.794933 \\
\hline 31 & 6 & 0 & -3.573530 & 1.922307 & -0.419466 \\
\hline 32 & 6 & 0 & 2.670654 & 0.547047 & 0.852502 \\
\hline 33 & 6 & 0 & 2.982642 & -0.218083 & 2.036034 \\
\hline 34 & 6 & 0 & 4.301635 & -0.598873 & 2.314846 \\
\hline 35 & 6 & 0 & 5.384195 & -0.279027 & 1.500662 \\
\hline 36 & 6 & 0 & 5.086305 & 0.419933 & 0.341082 \\
\hline 37 & 6 & 0 & 3.786937 & 0.823204 & -0.008424 \\
\hline 38 & 6 & 0 & 2.000642 & -0.638786 & 3.142572 \\
\hline 39 & 6 & 0 & 2.028065 & -2.147811 & 3.460602 \\
\hline 40 & 6 & 0 & 2.274475 & 0.173801 & 4.425977 \\
\hline 41 & 6 & 0 & 6.816870 & -0.637586 & 1.868031 \\
\hline 42 & 6 & 0 & 7.019873 & -2.151219 & 2.061275 \\
\hline 43 & 6 & 0 & 7.286056 & 0.140301 & 3.112963 \\
\hline 44 & 6 & 0 & 3.741160 & 1.552501 & -1.358215 \\
\hline 45 & 6 & 0 & 4.219520 & 0.652652 & -2.516017 \\
\hline 46 & 6 & 0 & 4.558157 & 2.859565 & -1.359046 \\
\hline 47 & 6 & 0 & 1.022185 & 2.974226 & -0.393148 \\
\hline 48 & 6 & 0 & 1.563567 & 4.105205 & 0.274419 \\
\hline 49 & 6 & 0 & 1.740277 & 5.314172 & -0.415685 \\
\hline 50 & 6 & 0 & 1.444130 & 5.443387 & -1.766837 \\
\hline 51 & 6 & 0 & 0.913178 & 4.327048 & -2.423644 \\
\hline 52 & 6 & 0 & 0.667353 & 3.111621 & -1.776207 \\
\hline 53 & 6 & 0 & 1.942877 & 4.094622 & 1.759843 \\
\hline 54 & 6 & 0 & 3.349862 & 4.650805 & 2.050031 \\
\hline 55 & 6 & 0 & 0.886866 & 4.845695 & 2.594509 \\
\hline 56 & 6 & 0 & 1.685969 & 6.755398 & -2.501275 \\
\hline 57 & 1 & 0 & 2.077496 & 7.467827 & -1.763033 \\
\hline 58 & 6 & 0 & 2.750871 & 6.603738 & -3.604021 \\
\hline 59 & 1 & 0 & 3.691214 & 6.221033 & -3.195238 \\
\hline 60 & 1 & 0 & 2.418843 & 5.909029 & -4.383419 \\
\hline 61 & 1 & 0 & 2.954329 & 7.567916 & -4.083046 \\
\hline 62 & 6 & 0 & 0.384165 & 7.350628 & -3.069567 \\
\hline 63 & 1 & 0 & -0.362544 & 7.492710 & -2.282250 \\
\hline 64 & 1 & 0 & 0.575185 & 8.322435 & -3.538132 \\
\hline 65 & 1 & 0 & -0.054059 & 6.696094 & -3.830653 \\
\hline 66 & 6 & 0 & -0.015988 & 1.988124 & -2.588324 \\
\hline 67 & 6 & 0 & -0.569910 & 2.440115 & -3.953079 \\
\hline 68 & 6 & 0 & 0.852946 & 0.739974 & -2.841563 \\
\hline 69 & 1 & 0 & -3.955383 & 1.130708 & 2.322819 \\
\hline 70 & 1 & 0 & -5.382900 & 1.012370 & 4.325133 \\
\hline 71 & 1 & 0 & -4.393242 & 0.483822 & 6.546786 \\
\hline 72 & 1 & 0 & -1.941598 & 0.083725 & 6.727765 \\
\hline 73 & 1 & 0 & -0.512382 & 0.228856 & 4.722529 \\
\hline 74 & 1 & 0 & -1.629854 & -3.307500 & -2.304129 \\
\hline 75 & 1 & 0 & -2.671994 & -2.326432 & -4.329195 \\
\hline 76 & 1 & 0 & -1.174581 & -2.554569 & -5.243376 \\
\hline 77 & 1 & 0 & -1.217382 & -1.457960 & -3.844494 \\
\hline 78 & 1 & 0 & -2.927358 & -4.840549 & -3.624848 \\
\hline 79 & 1 & 0 & -1.488610 & -5.713895 & -3.096301 \\
\hline 80 & 1 & 0 & -1.626187 & -5.134352 & -4.769908 \\
\hline 81 & 1 & 0 & 0.285415 & -4.055962 & -5.838719 \\
\hline 82 & 1 & 0 & 0.433598 & -5.738409 & -5.322481 \\
\hline 83 & 1 & 0 & 1.861077 & -4.829629 & -5.819687 \\
\hline 84 & 1 & 0 & 3.471423 & -5.468404 & -4.577101 \\
\hline 85 & 1 & 0 & 3.926528 & -5.782345 & -2.908692 \\
\hline
\end{tabular}




\begin{tabular}{|c|c|c|c|c|c|}
\hline 86 & 1 & 0 & 4.436520 & -4.284373 & -3.694183 \\
\hline 87 & 1 & 0 & 2.682208 & -2.721530 & -0.274730 \\
\hline 88 & 1 & 0 & 3.334254 & -4.705910 & 1.093658 \\
\hline 89 & 1 & 0 & 2.961538 & -5.779429 & -0.266851 \\
\hline 90 & 1 & 0 & 1.670559 & -4.828712 & 0.504615 \\
\hline 91 & 1 & 0 & 4.982535 & -3.167507 & -0.172958 \\
\hline 92 & 1 & 0 & 4.680147 & -2.778643 & -1.856017 \\
\hline 93 & 1 & 0 & 4.952220 & -4.468587 & -1.362758 \\
\hline 94 & 1 & 0 & -6.295525 & -0.816624 & -0.641037 \\
\hline 95 & 1 & 0 & -5.150862 & 3.266640 & -0.988889 \\
\hline 96 & 1 & 0 & 4.489107 & -1.148622 & 3.231975 \\
\hline 97 & 1 & 0 & 5.904225 & 0.674136 & -0.327799 \\
\hline 98 & 1 & 0 & 0.987055 & -0.433422 & 2.812973 \\
\hline 99 & 1 & 0 & 3.009973 & -2.499689 & 3.792667 \\
\hline 100 & 1 & 0 & 1.317506 & -2.361642 & 4.267122 \\
\hline 101 & 1 & 0 & 1.716319 & -2.729549 & 2.590086 \\
\hline 102 & 1 & 0 & 1.559208 & -0.085131 & 5.214756 \\
\hline 103 & 1 & 0 & 3.276032 & -0.030645 & 4.819016 \\
\hline 104 & 1 & 0 & 2.208638 & 1.251190 & 4.247457 \\
\hline 105 & 1 & 0 & 7.451122 & -0.325048 & 1.027366 \\
\hline 106 & 1 & 0 & 8.067287 & -2.374326 & 2.292515 \\
\hline 107 & 1 & 0 & 6.748049 & -2.708434 & 1.158959 \\
\hline 108 & 1 & 0 & 6.410672 & -2.534351 & 2.886850 \\
\hline 109 & 1 & 0 & 8.338329 & -0.073177 & 3.331909 \\
\hline 110 & 1 & 0 & 7.179013 & 1.219412 & 2.967137 \\
\hline 111 & 1 & 0 & 6.698587 & -0.136074 & 3.995273 \\
\hline 112 & 1 & 0 & 2.717431 & 1.830301 & -1.570317 \\
\hline 113 & 1 & 0 & 3.607686 & -0.249180 & -2.603267 \\
\hline 114 & 1 & 0 & 5.261631 & 0.341998 & -2.389274 \\
\hline 115 & 1 & 0 & 4.155265 & 1.192765 & -3.467885 \\
\hline 116 & 1 & 0 & 4.451795 & 3.367928 & -2.323877 \\
\hline 117 & 1 & 0 & 5.625746 & 2.675365 & -1.198071 \\
\hline 118 & 1 & 0 & 4.211129 & 3.545025 & -0.585171 \\
\hline 119 & 1 & 0 & 2.137403 & 6.175655 & 0.115171 \\
\hline 120 & 1 & 0 & 0.669603 & 4.426680 & -3.474571 \\
\hline 121 & 1 & 0 & 1.958111 & 3.056975 & 2.099527 \\
\hline 122 & 1 & 0 & 3.445482 & 5.707736 & 1.780216 \\
\hline 123 & 1 & 0 & 3.565527 & 4.569317 & 3.120980 \\
\hline 124 & 1 & 0 & 4.121023 & 4.092398 & 1.512785 \\
\hline 125 & 1 & 0 & 1.128231 & 4.796525 & 3.662462 \\
\hline 126 & 1 & 0 & 0.844923 & 5.903285 & 2.310166 \\
\hline 127 & 1 & 0 & -0.109256 & 4.419431 & 2.450595 \\
\hline 128 & 1 & 0 & -0.875467 & 1.658624 & -1.992378 \\
\hline 129 & 1 & 0 & 0.234301 & 2.646586 & -4.668998 \\
\hline 130 & 1 & 0 & -1.182083 & 1.639054 & -4.379484 \\
\hline 131 & 1 & 0 & -1.195203 & 3.332378 & -3.883006 \\
\hline 132 & 1 & 0 & 1.106713 & 0.193043 & -1.928647 \\
\hline 133 & 1 & 0 & 1.772997 & 1.005414 & -3.364936 \\
\hline 134 & 1 & 0 & 0.306429 & 0.042642 & -3.483397 \\
\hline 135 & 6 & 0 & -1.049138 & -3.546530 & 1.100659 \\
\hline 136 & 6 & 0 & -1.366914 & -4.754600 & 0.456815 \\
\hline 137 & 6 & 0 & -1.388766 & -3.444302 & 2.464148 \\
\hline 138 & 6 & 0 & -1.983732 & -5.810165 & 1.136516 \\
\hline 139 & 1 & 0 & -1.119096 & -4.891603 & -0.593417 \\
\hline 140 & 6 & 0 & -2.013526 & -4.484565 & 3.149259 \\
\hline 141 & 1 & 0 & -1.166207 & -2.516788 & 2.989257 \\
\hline 142 & 6 & 0 & -2.312191 & -5.678697 & 2.485963 \\
\hline 143 & 1 & 0 & -2.211077 & -6.734298 & 0.609906 \\
\hline 144 & 1 & 0 & -2.266223 & -4.366646 & 4.199860 \\
\hline 145 & 1 & 0 & -2.793011 & -6.495952 & 3.016234 \\
\hline 146 & 6 & 0 & -2.640894 & 3.120549 & -0.229366 \\
\hline
\end{tabular}




\begin{tabular}{rrrrrr}
147 & 6 & 0 & -4.070447 & -1.868727 & 0.234411 \\
148 & 1 & 0 & -3.007909 & -2.116612 & 0.275052 \\
149 & 6 & 0 & -2.948680 & 3.868277 & 1.084395 \\
150 & 1 & 0 & -2.275939 & 4.725904 & 1.195567 \\
151 & 1 & 0 & -3.976990 & 4.247664 & 1.083456 \\
152 & 1 & 0 & -2.826355 & 3.227555 & 1.959088 \\
153 & 6 & 0 & -4.760108 & -2.884227 & -0.698896 \\
154 & 1 & 0 & -4.379281 & -3.891288 & -0.501290 \\
155 & 1 & 0 & -5.843287 & -2.913462 & -0.537829 \\
156 & 1 & 0 & -4.599562 & -2.648344 & -1.754264 \\
157 & 6 & 0 & -4.617757 & -2.067064 & 1.663536 \\
158 & 1 & 0 & -4.518042 & -3.114779 & 1.963787 \\
159 & 1 & 0 & -4.082712 & -1.456849 & 2.392484 \\
160 & 1 & 0 & -5.678851 & -1.794700 & 1.711845 \\
161 & 6 & 0 & -7.294623 & 1.636829 & -1.347224 \\
162 & 1 & 0 & -7.301401 & 2.721269 & -1.520293 \\
163 & 6 & 0 & -7.685804 & 0.955802 & -2.672021 \\
164 & 1 & 0 & -8.681769 & 1.277627 & -2.996278 \\
165 & 1 & 0 & -6.973454 & 1.199142 & -3.466601 \\
166 & 1 & 0 & -7.706650 & -0.134303 & -2.565206 \\
167 & 6 & 0 & -8.330954 & 1.339981 & -0.247297 \\
168 & 1 & 0 & -8.391247 & 0.266836 & -0.036135 \\
169 & 1 & 0 & -8.072344 & 1.848613 & 0.686436 \\
170 & 1 & 0 & -9.328363 & 1.674525 & -0.553719 \\
171 & 6 & 0 & -2.697444 & 4.120486 & -1.400078 \\
172 & 1 & 0 & -1.860179 & 4.821141 & -1.337904 \\
173 & 1 & 0 & -2.651003 & 3.615003 & -2.367159 \\
174 & 1 & 0 & -3.619995 & 4.710938 & -1.381734 \\
175 & 1 & 0 & -1.614806 & 2.753302 & -0.156427 \\
----------------------------------------------------- \\
\hline
\end{tabular}

$E$ in a.u.: 5471.31373613 
Table S80. Imaginary frequencies and vibrational frequencies $\left(\mathrm{cm}^{-1}\right)$ of optimized structures at B3LYP/6-31G(d,p).

\begin{tabular}{|c|c|c|c|c|c|c|}
\hline & 9-Me & 10-Me & 11-Me & 15-Me & 16-Me & 17-Me \\
\hline \multirow{3}{*}{$\begin{array}{l}\text { Vibrational } \\
\text { Frequencies } \\
\text { Imaginary } \\
\text { Frequencies }\end{array}$} & 7.30 & 29.57 & 16.31 & 17.17 & 26.52 & 26.10 \\
\hline & 0 & 0 & 0 & 0 & 0 & 0 \\
\hline & 18-Me & 19-Me & 20-Me & 21-Me & 22-Me & 23-Me \\
\hline \multirow{3}{*}{$\begin{array}{l}\text { Vibrational } \\
\text { Frequencies } \\
\text { Imaginary } \\
\text { Frequencies }\end{array}$} & 7.34 & 19.22 & 57.02 & 52.33 & 11.26 & 14.45 \\
\hline & 0 & 0 & 0 & 0 & 0 & 0 \\
\hline & 24-Me & 25-Me & 26-Me & 27-Me & 28-Me & 29-Me \\
\hline \multirow{3}{*}{$\begin{array}{l}\text { Vibrational } \\
\text { Frequencies } \\
\text { Imaginary } \\
\text { Frequencies }\end{array}$} & 5.54 & 33.34 & 12.35 & 14.15 & 27.52 & 8.85 \\
\hline & 0 & 0 & 0 & 0 & 0 & 0 \\
\hline & 30-Me & $5 \mathrm{~b}-\mathrm{Me}$ & 6b-Me & 7-Me & 8-Me & NHC \\
\hline \multirow{3}{*}{$\begin{array}{l}\text { Vibrational } \\
\text { Frequencies } \\
\text { Imaginary } \\
\text { Frequencies }\end{array}$} & -20.65 & 21.29 & 22.52 & 9.40 & 23.64 & 125.81 \\
\hline & 1 & 0 & 0 & 0 & 0 & 0 \\
\hline & TS1 & TS2 & TS3 & TS4 & TS5 & TS6 \\
\hline \multirow{3}{*}{$\begin{array}{l}\text { Vibrational } \\
\text { Frequencies } \\
\text { Imaginary } \\
\text { Frequencies }\end{array}$} & -242.34 & -316.62 & -17.25 & -134.57 & -215.69 & -52.48 \\
\hline & 1 & 1 & 1 & 1 & 1 & 1 \\
\hline & TS7 & TS8 & TS9 & TS10 & TS11 & TS12 \\
\hline \multirow{3}{*}{$\begin{array}{l}\text { Vibrational } \\
\text { Frequencies } \\
\text { Imaginary } \\
\text { Frequencies }\end{array}$} & -51.38 & -131.09 & -164.61 & -184.22 & -181.12 & -25.29 \\
\hline & 1 & 1 & 1 & 1 & 1 & 1 \\
\hline & TS13 & TS14 & TS15 & TS16 & TS17 & \\
\hline \multirow{2}{*}{$\begin{array}{l}\text { Vibrational } \\
\text { Frequencies } \\
\text { Imaginary } \\
\text { Frequencies }\end{array}$} & -1053.74 & -193.30 & -185.09 & -421.22 & -247.05 & \\
\hline & 1 & 1 & 1 & 1 & 1 & \\
\hline
\end{tabular}


Table S81. Imaginary frequencies and vibrational frequencies $\left(\mathrm{cm}^{-1}\right)$ of optimized structures at B3LYP/6-31G(d,p).

\begin{tabular}{|c|c|c|c|c|c|c|}
\hline & 7-Ph & 8-Ph & 9-Ph & 12-Ph & 15-Ph & 16-Ph \\
\hline \multirow{3}{*}{$\begin{array}{l}\text { Vibrational } \\
\text { Frequencies } \\
\text { Imaginary } \\
\text { Frequencies }\end{array}$} & 21.63 & 22.12 & 31.31 & 7.13 & 13.89 & 7.98 \\
\hline & 0 & 0 & 0 & 0 & 0 & 0 \\
\hline & 17-Ph & 18-Ph & 19-Ph & 20-Ph & 22-Ph & 30-Ph \\
\hline \multirow{3}{*}{$\begin{array}{l}\text { Vibrational } \\
\text { Frequencies } \\
\text { Imaginary } \\
\text { Frequencies }\end{array}$} & 16.10 & 17.34 & 21.31 & 9.73 & 16.17 & 16.31 \\
\hline & 0 & 0 & 0 & 0 & 0 & 1 \\
\hline & 31-Ph & 32-Ph & 33-Ph & 34-Ph & 14-Ph & 35-Ph \\
\hline \multirow{3}{*}{$\begin{array}{l}\text { Vibrational } \\
\text { Frequencies } \\
\text { Imaginary } \\
\text { Frequencies }\end{array}$} & 6.01 & 3.92 & 8.17 & 17.17 & 12.63 & 15.44 \\
\hline & 0 & 0 & 0 & 0 & 0 & 0 \\
\hline & 36-Ph & CN-Ph & Ac-Ph & $\mathrm{NHC}^{\text {IPr2Me2 }}$ & & \\
\hline $\begin{array}{l}\text { Vibrational } \\
\text { Frequencies }\end{array}$ & 22.55 & 149.72 & 145.16 & 33.01 & & \\
\hline $\begin{array}{l}\text { Imaginary } \\
\text { Frequencies }\end{array}$ & 0 & 0 & 0 & 0 & & \\
\hline
\end{tabular}

\begin{tabular}{ccccccc}
\hline & TS1-Ph & TS2-Ph & TS3-Ph & TS4-Ph & TS5-Ph & TS9-Ph \\
\hline $\begin{array}{c}\text { Vibrational } \\
\text { Frequencies } \\
\text { Imaginary }\end{array}$ & -240.74 & -409.55 & -16.88 & -131.82 & -171.40 & -130.55 \\
Frequencies & 1 & 1 & 1 & 1 & 1 & 1 \\
\hline & TS17-Ph & TS18-Ph & TS19-Ph & TS20-Ph & TS21-Ph & TS22-Ph \\
\hline $\begin{array}{c}\text { Vibrational } \\
\text { Frequencies } \\
\text { Imaginary }\end{array}$ & -206.49 & -63.26 & -60.94 & -30.12 & -144.41 & 112.09 \\
Frequencies & 1 & 1 & 1 & 1 & 1 & 1 \\
\hline
\end{tabular}

\begin{tabular}{cc} 
& TS23-Ph \\
\hline Vibrational & -72.15 \\
Frequencies & \\
Imaginary & 1 \\
Frequencies &
\end{tabular}




\section{References}

[S1] Frisch, M. J.; Trucks, G. W.; Schlegel, H. B.; Scuseria, G. E.; Robb, M. A.; Cheeseman, J. R.; Scalmani, G.; Barone, V.; Mennucci, B.; Petersson, G. A.; Nakatsuji, H.; Caricato, M.; Li, X.; Hratchian, H. P.; Izmaylov, A. F.; Bloino, J.; Zheng, G.; Sonnenberg, J. L.; Hada, M.; Ehara, M.; Toyota, K.; Fukuda, R.; Hasegawa, J.; Ishida, M.; Nakajima, T.; Honda, Y.; Kitao, O.; Nakai, H.; Vreven, T.; Montgomery, J. A., Jr.; Peralta, J. E.; Ogliaro, F.; Bearpark, M.; Heyd, J. J.; Brothers, E.; Kudin, K. N.; Staroverov, V. N.; Kobayashi, R.; Normand, J.; Raghavachari, K.; Rendell, A.; Burant, J. C.; Iyengar, S. S.; Tomasi, J.; Cossi, M.; Rega, N.; Millam, J. M.; Klene, M.; Knox, J. E.; Cross, J. B.; Bakken, V.; Adamo, C.; Jaramillo, J.; Gomperts, R.; Stratmann, R. E.; Yazyev, O.; Austin, A. J.; Cammi, R.; Pomelli, C.; Ochterski, J. W.; Martin, R. L.; Morokuma, K.; Zakrzewski, V. G.; Voth, G. A.; Salvador, P.; Dannenberg, J. J.; Dapprich, S.; Daniels, A. D.; Farkas, Ö.; Foresman, J. B.; Ortiz, J. V.; Cioslowski, J.; Fox, D. J. Gaussian, Inc., Wallingford CT, 2009.Gaussian 09, Revision A.02, 2009.

[S2] Fukui, K. Acc. Chem. Res. 1981, 14, 363.

[S3] Gonzalez, C.; Schlegel, H.; J. Chem. Phys. 1991, 95, 5853.

[S4] Dennington, Roy; Keith, Todd; Millam, John., Semichem Inc., Shawnee Mission, KS, GaussView, Version 5, 2009. 